\title{
Thermodynamic Representations of Ammonia and Isobutane
}

\author{
S. L. Milora \\ S. K. Combs
}

\section{OAK RIDGE NATIONAL LABORATORY}




\section{DISCLAIMER}

This report was prepared as an account of work sponsored by an agency of the United States Government. Neither the United States Government nor any agency Thereof, nor any of their employees, makes any warranty, express or implied, or assumes any legal liability or responsibility for the accuracy, completeness, or usefulness of any information, apparatus, product, or process disclosed, or represents that its use would not infringe privately owned rights. Reference herein to any specific commercial product, process, or service by trade name, trademark, manufacturer, or otherwise does not necessarily constitute or imply its endorsement, recommendation, or favoring by the United States Government or any agency thereof. The views and opinions of authors expressed herein do not necessarily state or reflect those of the United States Government or any agency thereof. 


\section{DISCLAIMER}

Portions of this document may be illegible in electronic image products. Images are produced from the best available original document. 


\section{Printed in the United States of America. Available from National Technical Information Service \\ U.S. Department of Commerce 5285 Port Royal Road, Springfield, Virginia 22161 \\ Price: Printed Copy $\$ 5.50$; Microfiche $\$ 3.00$}

This report was prepared as an account of work sponsored by the United States Government. Neither the United States nor the Energy Research and Development Administration/United States Nuclear Regulatory Commission, nor any of their employees, nor any of their contractors, subcontractors, or their employees, makes any warranty, express or implied, or assumes any legal liability or responsibility for the accuracy, completeness or usefulness of any information, apparatus, product or process disclosed, or represents that its use would not infringe privately owned rights. 
Contract No. W-7405-eng-26

Engineering Technology Division

THERMODYNAMIC REPRESENTATIONS OF AMMONIA AND ISOBUTANE

S. L. Milora and S. K. Combs

Date Published - May 1977

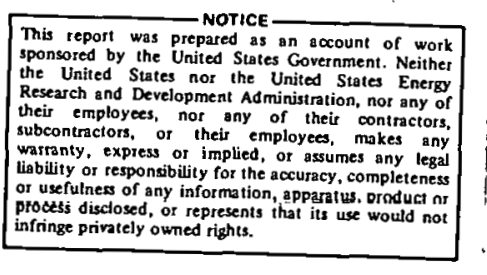

Prepared by

OAK RIDGE NATIONAL LABORATORY

Oak Ridge, Tennessee 37830

operated by

UNION CARBIDE CORPORATION

for the

ENERCY RESEARCH AND DEVELOPMENT ADMINISTRATION 


\section{THIS PAGE}

\section{WAS INTENTIONALLY \\ LEFT BLANK}


CONTENTS

$\underline{\text { Page }}$

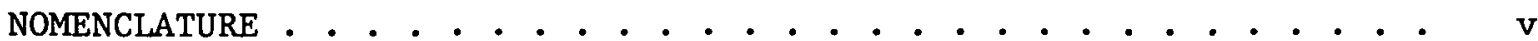

ABSTRACT . . . . . . . . . . . . . . . . . . . . . . 1

1. INTRODUCTION . . . . . . . . . . . . . . . . . . . 1

2. THE THERMODYNAMIC REPRESENTATION . . . . . . . . . . . . . 2

2.1 Superheated Vapor Conditions . . . . . . . . . . 2

2.2 Liquid-Vapor Equilibrium Conditions . . . . . . . . . 5

2.2.1 Liquid density and vapor pressure equations . . . 5

2.2.2 The latent heat of vaporization .......... 5

2.3 The Ideal-Gas State................. 6

2.4 Derived Thermodynamic Properties . . . . . . . . 6

3. ESTIMATION OF ERRORS . . . . . . . . . . . . . . . . . . . 7

3.1 Superheated Vapor and Dense Gaseous Regions . . . . . . . 7

3.2 Vapor Pressure and Saturated Liquid Volume . . . . . . 19

4. THERMODYNAMIC PROPERTIES TABLES . . . . . . . . . . . . 23

REFERENCES . . . . . . . . . . . . . . . . . . . . . 98 
THIS PAGE

WAS INTENTIONALLY

LEFT BLANK 


\section{NOMENCLATURE}

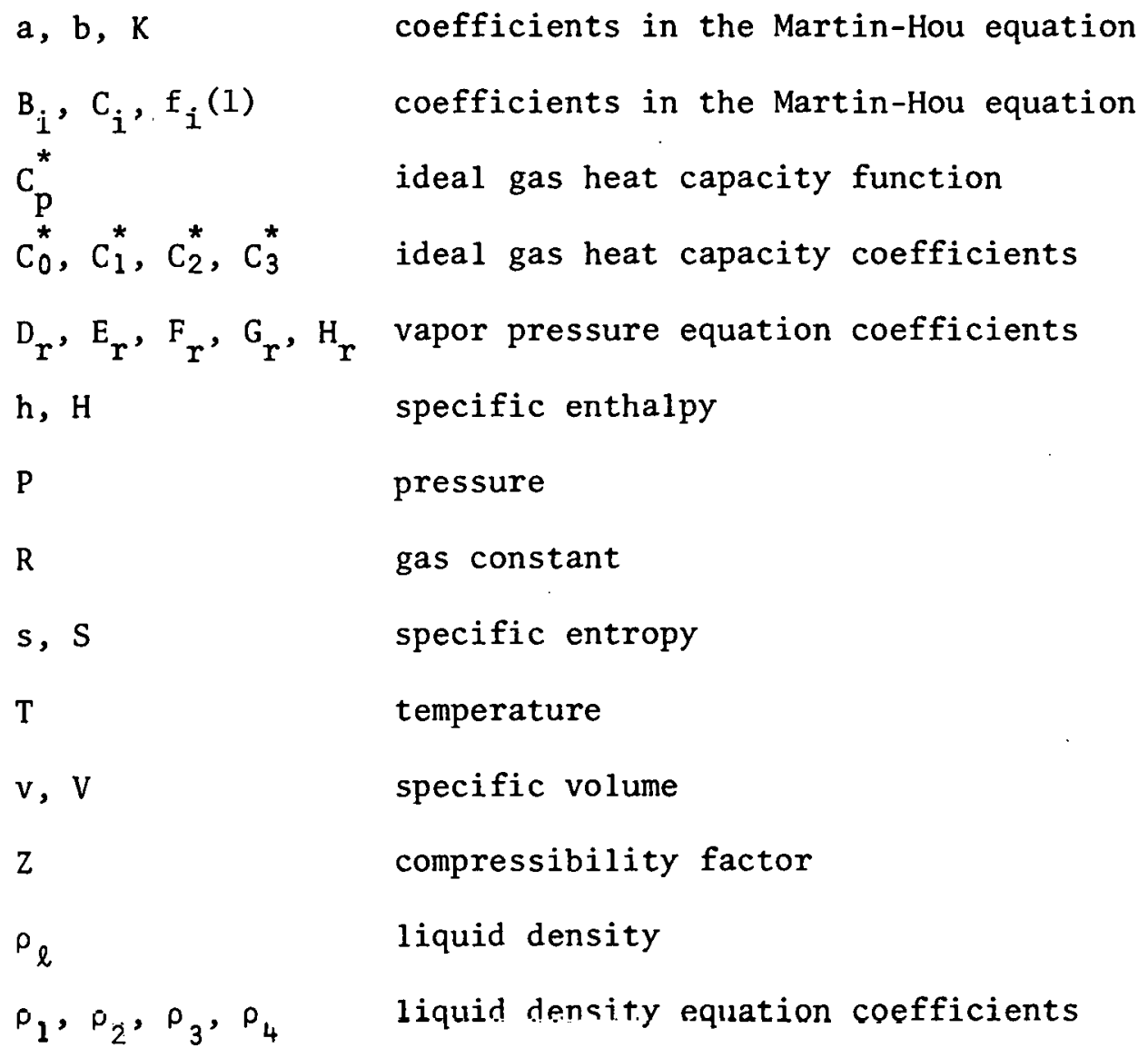




\title{
THERMODYNAMIC REPRESENTATIONS OF AMMONIA AND ISOBUTANE
}

\author{
S. L. Milora and S. K. Combs
}

\begin{abstract}
Tables of the thermodynamic properties of ammonia and isobutane are presented for the superheated vapor and the saturated liquid and vapor states. The properties were calculated using appropriate analytical pressure-volume-temperature (P-V-T) representations for the fluids in the regions described.

The tables cover the approximate range of values of reduced temperatures up to 1.5 and reduced pressure up to 5 .
\end{abstract}

\section{INTRODUCTION}

This report represents a compilation of the thermodynamic properties of ammonia and isobutane which have been calculated from analytic thermodynamic representations for the two fluids in the liquid-vapor equilibrium st.ates and the superheated vapor and compressed gaseous regions. The equation of state upon which the formulation is based is a revised Martin-Hou representation ${ }^{1}$ that is particularly well-suited to describing $\mathrm{P}-\mathrm{V}-\mathrm{T}$ behavior of fluids at supercritical pressures. This feature is important in some applications such as power cycle calculations for thermodynamic cycles employing supercritical pressure boilers. ${ }^{2}$ The representations fur both fluids described here are valid up to values of reduced pressures of about 5 and reduced temperatures of about 1.5 , which is more than adequate for most applications. The subcooled liquid region is excluded, since both representations are inaccurate at values of reduced density greater than unity for temperatures less than the critical value. 


\section{THE THERMODYNAMIC REPRESENTATION}

\subsection{Superheated Vapor Conditions}

In the superheated vapor and high density (supercritical) compressed gaseous regions, the P-V-T behavior of the fluids is described by the following dimensionless, or reduced, form of the relevant Martin-Hou equation: ${ }^{1,3}$

$$
\begin{aligned}
P_{r}=\frac{P}{P_{c}}= & z_{c}^{-i}\left\{\frac{T_{r}}{\left(v_{r}-b\right)}+\frac{f_{1}\left(T_{r}\right)}{\left(v_{r}-b\right)^{2}}+\frac{f_{2}\left(T_{r}\right)}{\left(v_{r}-b\right)^{3}}+\frac{f_{3}\left(T_{r}\right)}{\left(v_{r}-b\right)^{4}}+\right. \\
& \left.\frac{f_{4}\left(T_{r}\right)}{\left(v_{r}-b\right)^{5}}+f_{5}\left(T_{r}\right) e^{-a v_{r}}+f_{6}\left(T_{r}\right) e^{-2 a v_{r}}\right\},
\end{aligned}
$$

where $P_{r}=P / P_{c}, T_{r}=T / T$, and $v_{r}=v / v_{c}$ represent, respectively, reduced values of pressure, temperature, and specific volume. The subscript $c$ pertains to values at the thermodynamic critical point, and $z_{c}$ is the critical compressibility factor defined as $Z_{c}=\left(P_{c} v_{c}\right) / R T$, where $R$ is the fluid specific gas constant in consistent units. The temperature functions of Eq. (1) [which are related to the virial coefficients] are written in the following dimensionless form:

$$
f_{i}\left(T_{r}\right)=f_{i}(1)+B_{i}\left(T_{r}-1\right)+C_{i}\left(e^{-K T_{r}}-e^{-K}\right),
$$

where $B_{i}, C_{i}$, and $K$ are constants and $f_{\dot{i}}(1)$ represents the value of the relevant temperature function evaluated at the critical temperature.

Milora ${ }^{3,4}$ has determined values of the parameters $f_{i}(1), B_{i}, C_{i}, K$, $a$, and $b$ for ammonia and isobutane by using a nonlinear least-squares algorithm to fit the available P-V-T data. The results are listed in Tables 1 and 2 along with other pertinent physical constants. 
Table 1. Equation-of-state parameters for ammonia

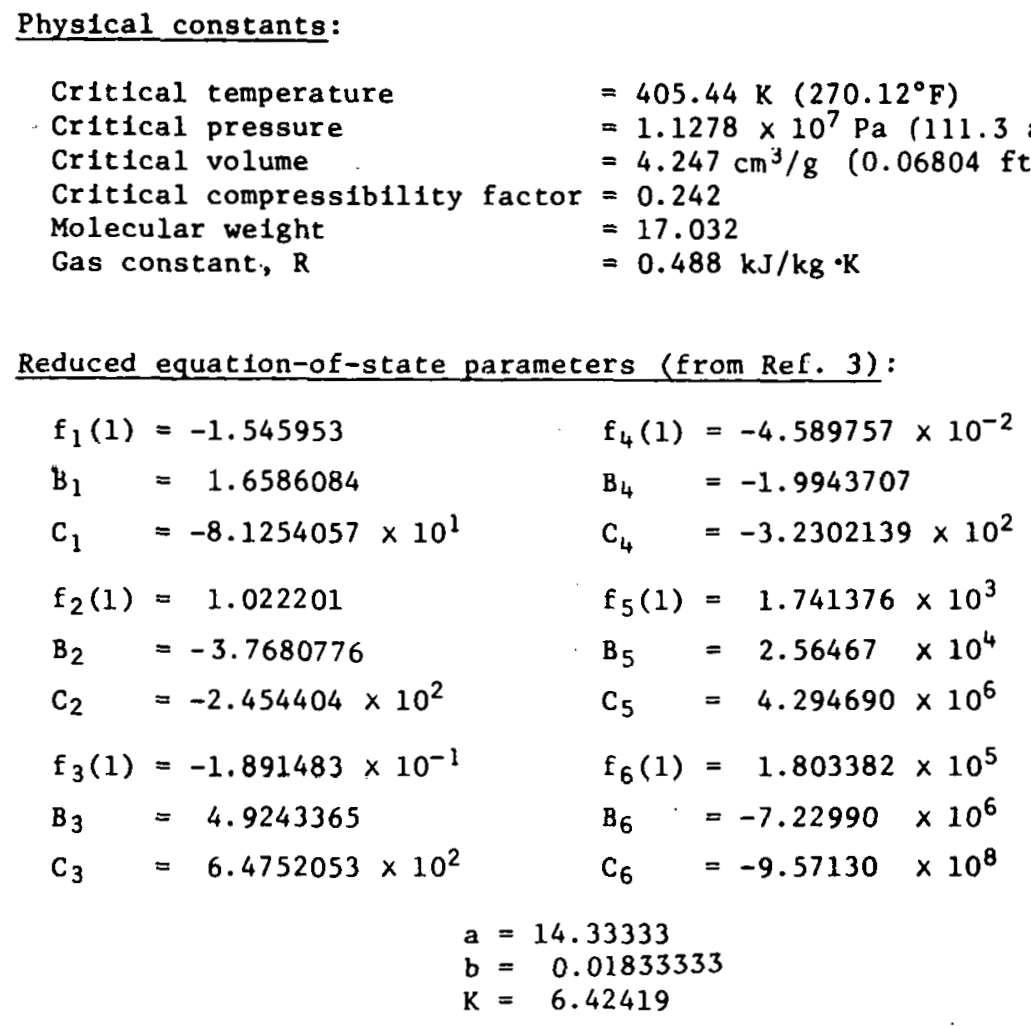

Ideal-gas heat capacity coefficients (reduced):

$$
\begin{array}{cc}
C_{0}^{*}=4.30317 & C_{2}^{*}=3.1995 \\
C_{1}^{*}=-1.84109 & C_{3}^{*}=-0.982953 \\
& C_{\mathrm{p}}^{*} / R=C_{0}^{*}+C_{1}^{*} T_{r}+C_{2}^{*} T_{r}^{2}+C_{3}^{*} T_{r}^{3}
\end{array}
$$

Liquid density equation coefficients (reduced):

$$
\begin{aligned}
& \rho_{1}=1.5489388 \\
& \rho_{3}=-1.5957075 \\
& \rho_{2}=1.9710952 \quad \rho_{4}=1.0321973 \\
& \left.\rho_{\ell}\right)_{r}^{s a t}=1+\rho_{1}\left(1-T_{r}\right)^{1 / 3}+\rho_{2}\left(1-T_{r}\right)^{2 / 3}+\rho_{3}\left(1-T_{r}\right)+\rho_{4}\left(1-T_{r}\right)^{4 / 3}
\end{aligned}
$$

Vapor pressure equation coefficients (reduced):

$$
\begin{array}{cc}
D_{r}=7.3672454 & F_{r}=8.5673754 \\
E_{r}=10.883945 & G_{r}=-7.4647451 . \\
H_{r}=1.0519545 \\
10 g P)_{r}^{s a t}=D_{r}-E_{r} / T_{r}-F_{r} \ln T_{r}-G_{r} T_{r}+H_{r} T_{r}^{2}
\end{array}
$$


Table 2. Equation-of-state parameters for 1sobutane

Physical constants:

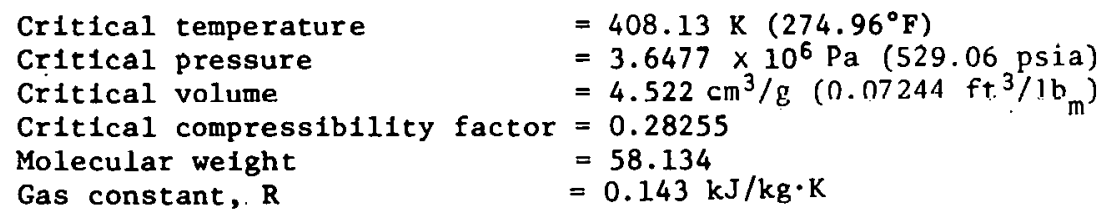

Reduced equation-of-state parameters (from Ref. 4):

$\begin{array}{lll}\mathrm{f}_{1}(1)=-1.3452811 & \mathrm{f}_{4}(1)=-3.6735063 \times 10^{-3} \\ \mathrm{~B}_{1}=5.6584041 \times 10^{-1} & \mathrm{~B}_{4}=6.7988776 \times 10^{-2} \\ \mathrm{C}_{1}=-1.2094862 \times 10^{1} & \mathrm{C}_{4}=-1.3506749 \\ \mathrm{f}_{2}(1)=8.1331706 \times 10^{-1} & \mathrm{~F}_{5}(1)=7.3698560 \times 10^{3} \\ \mathrm{~B}_{2}=1.9346061 \times 10^{-1} & \mathrm{~B}_{5}=-1.6476544 \times 10^{4} \\ \mathrm{C}_{2}=1.5083697 \times 10^{1} & \mathrm{C}_{5}=2.0091059 \times 10^{5} \\ \mathrm{f}_{3}(1)=-1.0209186 \times 10^{-1} & \mathrm{f}_{6}(1)=4.1434338 \times 10^{6} \\ \mathrm{~B}_{3}=-1.8524754 \times 10^{-1} & \mathrm{~B}_{6}=1.4277603 \times 10^{7} \\ \mathrm{C}_{3}=-2.0223683 \times & \mathrm{C}_{6}=5.4888339 \times 10^{8}\end{array}$

$$
\begin{aligned}
& a=16.024343 \\
& b=0.14926478 \\
& K=3.9676634
\end{aligned}
$$

Ideal-gas heat capacity coefficients (reduced):

$$
\begin{aligned}
& \mathrm{c}_{0}^{*}=-0.95123056 \quad \mathrm{c}_{2}^{*}=-4.6066_{4} \\
& \mathrm{l}_{1}^{*}=211.41191 \quad \mathrm{C}_{3}^{*}=0.407839 \\
& C_{p}^{*} / R=C_{0}^{*}+C_{1}^{*} T_{r}+C_{2}^{*} \mathrm{~T}_{r}^{2}+C_{3}^{*} T_{r}^{3}
\end{aligned}
$$

Liquid density equation coefficients (reduced):

$$
\begin{array}{lrl}
\rho_{1}=1.17235 & \rho_{3}=\cdot 7.50505 \\
\rho_{2}=4.61147 & \rho_{4}=4.76730 \\
\left.\rho_{\ell}\right)_{r}^{\text {sat }}=1+\rho_{1}\left(1-T_{r}\right)^{1 / 3}+\rho_{2}\left(1-T_{r}\right)^{2 / 3}+\rho_{3}\left(1-T_{r}\right)+\rho_{4}\left(1-T_{r}\right)^{4 / 3}
\end{array}
$$

Vapor pressure equation coefficients (reduced):

$$
\begin{aligned}
& \mathrm{D}_{\mathrm{r}}=10,890498 \\
& E_{r}=8.7655777 \\
& F_{r}=2.6561239 \\
& G_{r}=4.7799233 \\
& H_{r}=2.6350034 \\
& \log P)_{r}^{\text {sat }}=D_{r}-E_{r} / T_{r}-F_{r} \text { en } T_{r}-G_{r} T_{r}+H_{r} T_{r}^{2}
\end{aligned}
$$




\subsection{Liquid-Vapor Equilibrium Conditions}

\subsubsection{Liquid density and vapor pressure equations}

The following standard forms were chosen to represent the saturated reduced liquid density and the reduced vapor pressure, respectively:

$$
\begin{aligned}
\left.\rho_{\ell}\right)_{\mathrm{r}}^{\mathrm{sat}=} & 1+\rho_{1}\left(1-\mathrm{T}_{\mathrm{r}}\right)^{1 / 3}+\rho_{2}\left(1-\mathrm{T}_{\mathrm{r}}\right)^{2 / 3}+\rho_{3}\left(1-\mathrm{T}_{\mathrm{r}}\right)+\rho_{4}\left(1-\mathrm{T}_{\mathrm{r}}\right)^{4 / 3}, \\
& \log \mathrm{P})_{\mathrm{r}}^{\mathrm{Sat}}=\mathrm{D}_{\mathrm{r}}-\mathrm{E}_{\mathrm{r}} / \mathrm{T}_{\mathrm{r}}-\mathrm{F}_{\mathrm{r}} \ln \mathrm{T}_{\mathrm{r}}-\mathrm{G}_{\mathrm{r}} \mathrm{T}_{\mathrm{r}}+\mathrm{H}_{\mathrm{r}} \mathrm{T}_{\mathrm{r}}^{2} .
\end{aligned}
$$

The form of Eq. (3) is consistent with the definition of the critical point; i.e., $\rho_{r}=1$ at $T_{r}=1$. Consistency is forced on Eq. (4) by requiring that $P_{r}=1$ at $T_{r}=1$. Values of the coefficients of Eqs. (3) and (4) as determined by a least-squares fit to the data are presented in Tables 1 and 2 .

\subsubsection{The latent heat of vaporization}

The latent heat of vaporization, $h_{f g}$, is calculated from the Clapeyron equation using the preceding results. In terms of reduced variables, the expression for $h_{f g}$ becomes

$$
\frac{h_{f g}}{R_{c}}=z_{c} T_{r}\left(v_{g}-v_{f}\right)_{r}^{s a t} \frac{d_{r}^{s a t}}{d_{r}},
$$

with

$$
\left.\left.v_{g}\right)_{r}^{s a t}=1 / \rho_{\ell}\right)_{r}^{s a t}
$$

The subscripts $g$ and $f$ refer to the gaseous and liquid states, respectively. It follows that the entropy variation, $s_{f g}$, during the phase change is given by

$$
\frac{s_{f g}}{R}=\frac{h_{f g}}{R_{C} T_{r}}
$$




\subsection{The Ideal-Gas State}

The properties of the gas at large values of reduced volume are required to complete the thermodynamic representation. In the limit $v_{r} \rightarrow \infty$, Eq. (1) becomes

$$
\mathrm{P}_{\mathrm{r}}=\frac{1}{\mathrm{Z}_{\mathrm{c}}} \frac{\mathrm{T}_{\mathrm{r}}}{\mathrm{v}_{\mathrm{r}}}
$$

which is the reduced form of the ideal-gas law. In this dilute state the enthalpy is a function only of temperature, as is the constant pressure specific heat function $C_{p}^{*}$. The latter is represented accurately over the temperature range of interest by a simple polynomial function

$$
\frac{\mathrm{C}_{\mathrm{p}}^{*}}{\mathrm{R}}=\mathrm{C}_{0}^{*}+\mathrm{C}_{1}^{*} \mathrm{~T}_{\mathrm{r}}+\mathrm{C}_{2}^{*} \mathrm{~T}_{\mathrm{r}}^{2}+\mathrm{C}_{\mathrm{3}}^{*} \mathrm{~T}_{\mathrm{r}}^{3} \text {. }
$$

The relevant dimensionless coefficients are given in Tables 1 and 2 .

\subsection{Derived Thermodynamic Properties}

The derived thermodynamic properties, including enthalpy and entropy, are expressible as algebraic relationships involving the primary thermodynamic variables, the equation-of-state parameters, and the ideal-gas specific heat coefficients. For changes in the values of enthalpy and entropy between the state points $\mathrm{P}_{1}, \mathrm{~T}_{1}$ and $\mathrm{P}_{2}, \mathrm{~T}_{2}$, the following expressions apply: ${ }^{3}$

$$
\begin{aligned}
& \frac{h_{2}\left(P_{2}, T_{2}\right)-h_{1}\left(P_{1}, T_{1}\right)}{R_{c}}=\left.\left\{C_{0}^{*} T_{r}+C_{1}^{*} \frac{T_{r}^{2}}{2}+C_{2}^{*} \frac{T_{r}^{3}}{3}+C_{3}^{*} \frac{T_{r}^{4}}{4}\right\}\right|_{T_{r}} ^{T_{r_{2}}} \\
& +\left\{Z_{c} P_{r} v_{r}-T_{r}+\sum_{i=1}^{4} \frac{f_{i}(1)-B_{i}+C_{i}\left[\left(1+K T_{r}\right) c^{-K T_{r}}-c^{-K}\right]}{i\left(v_{r}-b\right)}\right. \\
& +\frac{f_{5}(1)-B_{5}+C_{5}\left[\left(1+K T_{r}\right) e^{-K T_{r}}-e^{\left.-K_{1}\right]}\right.}{a e^{a v_{r}}}
\end{aligned}
$$




$$
\begin{aligned}
& \left.+\frac{f_{6}(1)-B_{6}+C_{6}\left[\left(1+K T_{r}\right) e^{-K T} r-e^{-K}\right]}{2 a e^{2 a v_{r}}}\right\} \mid \begin{array}{l}
v_{r_{2}}, T_{r_{2}} \\
v_{r_{1},}, T_{r_{1}}
\end{array} \\
& \frac{s_{2}-s_{1}}{R}=\left.\left\{\begin{array}{ll}
C_{0}^{*} \ln T_{r}+C_{1}^{*} T_{r}+C_{2}^{*} \frac{T_{r}^{2}}{2}+C_{3}^{*} \frac{T_{r}^{3}}{3}-\ln \cdot P_{r}
\end{array}\right\}\right|_{P_{r_{1}}, T_{r_{1}}} ^{{ }_{r_{2}}, T_{r_{2}}} \\
& -\left\{l n \cdot \frac{\mathrm{T}_{r}}{\left(v_{r}-b\right) P_{r} z_{c}}+\sum_{i=1}^{4} \frac{B_{i}-C_{i} K e^{-K T_{r}}}{i\left(v_{r}-b\right)^{i}}\right. \\
& \left.+\frac{B_{5}-C_{5} K e^{-K T_{r}}}{a e^{a v_{r}}}+\frac{B_{6}-C_{6} K e^{-K T_{r}}}{2 a e^{2 a v_{r}}}\right\}\left.\right|_{T_{r_{1}}, v_{r}\left(T_{r_{1}}, P_{r_{1}}\right)} ^{T_{r_{2}}, v_{r}\left(T_{r_{2}}, P_{r_{2}}\right)}
\end{aligned}
$$

\section{ESTIMATION OF ERRORS}

\subsection{Superheated Vapor and Dense Gaseous Regions}

A detailed discussion of the accuracy of the P-V-T representation for ammonia appears in an earlier report by Milora. ${ }^{3}$ Tables 3,4 , and 5 , which are taken from that work, summarize the comparison of the representation with the data of several authors. The largest discrepencies are confined to a small region in the vicinity of the critical point, where experimental errors are frequently large. Outside of this region, the errors are sma11 - typically of the order of a few tenths of a percent or less.

A partial listing of the errors encountered in the isobutane representation is given in Table 6 for selected isotherms. Again, the largest discrepancies occur along the isotherm nearest the critical temperature; however, the errors are typically greater in magnitude than those experienced for ammonia. As is true for ammonia, the P-V-T domain external to this region is represented accurately. 
Table 3. Results of anmonia P-V-T representation: Comparison with data of Davies 5

\begin{tabular}{|c|c|c|c|c|c|c|c|}
\hline $\begin{array}{l}\mathrm{T}, \\
\mathrm{K}\end{array}$ & $\mathrm{cm}^{\mathrm{v}} ; \mathrm{g}$ & $\begin{array}{c}\mathrm{P}_{\text {exp }} \\
\mathrm{Pa} \times 10^{-5}\end{array}$ & $\begin{array}{c}\mathrm{P}_{\mathrm{Ca} 1} \\
\mathrm{~Pa} \times 10^{-5}\end{array}$ & $(\Delta \mathrm{P} / \mathrm{P}) \times 100$ & $\begin{array}{l}\mathrm{h}_{\exp } \\
\mathrm{kJ} / \mathrm{kg}\end{array}$ & $\begin{array}{l}\mathrm{h}_{\mathrm{cal}}, \\
\mathrm{kJ} / \mathrm{kg}\end{array}$ & $(\Delta h / h) \times 100$ \\
\hline $\begin{array}{l}360 \\
360 \\
360\end{array}$ & $\begin{array}{r}164.3 \\
77.0 \\
32.3\end{array}$ & $\begin{array}{l}10.133 \\
20.265 \\
40.530\end{array}$ & $\begin{array}{l}10.124 \\
20.263 \\
40.563\end{array}$ & $\begin{array}{r}-0.08 \\
-0.01 \\
0.08\end{array}$ & $\begin{array}{l}1630.1 \\
1593.2 \\
1494.2\end{array}$ & $\begin{array}{l}1629.4 \\
1590.8 \\
1492.0\end{array}$ & $\begin{array}{l}-0.04 \\
-0.16 \\
-0.15\end{array}$ \\
\hline $\begin{array}{l}400 \\
400 \\
400 \\
400 \\
400 \\
400\end{array}$ & $\begin{array}{r}185.9 \\
89.3 \\
40.5 \\
24.1 \\
15.1 \\
8.1\end{array}$ & $\begin{array}{r}10.133 \\
20.265 \\
40.530 \\
60.795 \\
81.061 \\
101.330\end{array}$ & $\begin{array}{r}10.130 \\
20.267 \\
40.494 \\
60.804 \\
80.793 \\
101.530\end{array}$ & $\begin{array}{r}-0.03 \\
0.01 \\
-0.09 \\
0.15 \\
-0.33 \\
0.20\end{array}$ & $\begin{array}{l}1729.3 \\
1703.8 \\
1644.1 \\
1572.6 \\
1479.3 \\
1267.5\end{array}$ & $\begin{array}{l}1727.7 \\
1700.5 \\
1640.2 \\
1567.4 \\
1472.9 \\
1279.3\end{array}$ & $\begin{array}{r}-0.09 \\
-0.19 \\
-0.24 \\
-0.33 \\
-0.43 \\
0.93\end{array}$ \\
\hline $\begin{array}{l}420 \\
420 \\
420 \\
420 \\
420 \\
420 \\
420 \\
420 \\
420 \\
420\end{array}$ & $\begin{array}{l}95.1 \\
44.3 \\
27.1 \\
18.2 \\
13.6 \\
5.50 \\
2.02 \\
2.48 \\
2.28 \\
2.18\end{array}$ & $\begin{array}{r}20.265 \\
40.530 \\
60.795 \\
81.060 \\
101.330 \\
141.860 \\
182.390 \\
243.180 \\
324.240 \\
405.300\end{array}$ & $\begin{array}{r}20.267 \\
40.519 \\
60.800 \\
81.230 \\
100.050 \\
139.110 \\
183.240 \\
242.320 \\
328.310 \\
409.440\end{array}$ & $\begin{array}{r}0.01 \\
-0.03 \\
0.01 \\
0.21 \\
-1.26 \\
-1.93 \\
0.47 \\
-0.36 \\
1.25 \\
1.02\end{array}$ & $\begin{array}{r}1755.4 \\
1704.8 \\
1649.3 \\
1586.6 \\
1508.3 \\
1196.5 \\
988.7 \\
936.9 \\
709.1 \\
395.6\end{array}$ & $\begin{array}{r}1752.4 \\
1702.2 \\
1644.9 \\
1577.5 \\
1501.4 \\
1196.1 \\
979.0 \\
928.3 \\
898.5 \\
884.4\end{array}$ & $\begin{array}{l}-0.14 \\
-0.15 \\
-0.26 \\
-0.57 \\
-0.46 \\
-0.04 \\
-0.98 \\
-0.91 \\
-1.17 \\
-1.25\end{array}$ \\
\hline $\begin{array}{l}480 \\
480 \\
480 \\
480 \\
480 \\
480 \\
480\end{array}$ & $\begin{array}{r}227.0 \\
111.4 \\
53 . \overline{6} \\
34.3 \\
24.6 \\
18.8 \\
12.0\end{array}$ & $\begin{array}{r}10.133 \\
20.265 \\
40.530 \\
60.795 \\
81.061 \\
101.330 \\
141.860\end{array}$ & $\begin{array}{r}10.138 \\
20.278 \\
40.560 \\
60.819 \\
81.019 \\
100.930 \\
141.120\end{array}$ & $\begin{array}{r}0.06 \\
0.06 \\
0.07 \\
0.04 \\
-0.05 \\
-0.39 \\
-0.52\end{array}$ & $\begin{array}{l}1726.3 \\
1710.1 \\
1376.7 \\
1341.8 \\
1805.5 \\
1767.7 \\
1683.4\end{array}$ & $\begin{array}{l}1924.6 \\
1907.7 \\
1873.0 \\
1837.1 \\
1799.9 \\
1761.5 \\
1677.7\end{array}$ & $\begin{array}{l}-0.09 \\
-0.13 \\
-0.19 \\
-0.26 \\
-0.31 \\
-0.35 \\
-0.34\end{array}$ \\
\hline $\begin{array}{l}480 \\
480 \\
480 \\
480\end{array}$ & $\begin{array}{l}8 . .2 \\
5.02 \\
3.50 \\
2.93\end{array}$ & $\begin{array}{l}182.390 \\
243.180 \\
324.240 \\
405.300\end{array}$ & $\begin{array}{l}182.120 \\
243.760 \\
323.680 \\
408.010\end{array}$ & $\begin{array}{r}-0.15 \\
0.24 \\
-0.17 \\
0.67\end{array}$ & $\begin{array}{l}1588.6 \\
1439.7 \\
1307.3 \\
1241.0\end{array}$ & $\begin{array}{l}1582.8 \\
1437.3 \\
1309.6 \\
1243.9\end{array}$ & $\begin{array}{r}-0.39 \\
-0.16 \\
0.17 \\
0.23\end{array}$ \\
\hline
\end{tabular}


Table 4. Results of ammonia P-V-T representation: Comparison with data of Beattie and Lawrence ${ }^{6}$

\begin{tabular}{|c|c|c|c|c|c|c|c|c|}
\hline $\begin{array}{l}\mathrm{T}, \\
\mathrm{K}\end{array}$ & $\begin{array}{c}\mathrm{v}, \\
\mathrm{cm}^{3} / \mathrm{g}\end{array}$ & $\begin{array}{c}\mathrm{P}_{\text {exp }} \\
\mathrm{Pa} \times 10^{-5} \\
\end{array}$ & $\begin{array}{r}\mathrm{P}_{\mathrm{cal}}{ }^{\prime} \\
\mathrm{Pa} \times 10^{-5} \\
\end{array}$ & $(\Delta \mathrm{P} / \mathrm{P}) \times 100$ & $\begin{array}{l}h_{\exp },^{a} \\
k J / k g\end{array}$ & $\begin{array}{l}\mathrm{h}_{\mathrm{cal}}, \\
\mathrm{kJ} / \mathrm{kg}\end{array}$ & $(\Delta \mathrm{h} / \mathrm{h})$ & $\times 100^{a}$ \\
\hline $\begin{array}{l}323.1 \\
323.1\end{array}$ & $\begin{array}{l}90 \\
70\end{array}$ & $\begin{array}{l}15.250 \\
18.786\end{array}$ & $\begin{array}{l}15.254 \\
18.796\end{array}$ & $\begin{array}{l}0.03 \\
0.06\end{array}$ & - & $\begin{array}{l}1506.2 \\
1483.9\end{array}$ & & - \\
\hline $\begin{array}{l}373.1 \\
373.1 \\
373.1 \\
373.1 \\
373.1 \\
373.1\end{array}$ & $\begin{array}{l}90 \\
70 \\
50 \\
40 \\
30 \\
20\end{array}$ & $\begin{array}{l}18.482 \\
23.143 \\
30.874 \\
36.954 \\
45.789 \\
59.053\end{array}$ & $\begin{array}{l}18.47 \mathrm{C} \\
23.122 \\
30.831 \\
36.903 \\
45.708 \\
58.846\end{array}$ & $\begin{array}{l}-0.06 \\
-0.09 \\
-0.14 \\
-0.13 \\
-0.18 \\
-0.35\end{array}$ & $\begin{array}{l}- \\
- \\
- \\
- \\
-\end{array}$ & $\begin{array}{l}1634.1 \\
1617.6 \\
1588.1 \\
1562.6 \\
1521.0 \\
1440.6\end{array}$ & & $\begin{array}{l}- \\
- \\
- \\
- \\
-\end{array}$ \\
\hline $\begin{array}{l}398.1 \\
398.1 \\
398.1 \\
398.1 \\
398.1 \\
398.1\end{array}$ & $\begin{array}{l}90 \\
70 \\
50 \\
40 \\
30 \\
20\end{array}$ & $\begin{array}{l}20.012 \\
25.179 \\
33.883 \\
40.895 \\
51.413 \\
68.456\end{array}$ & $\begin{array}{l}20.00 \pi \\
25.163 \\
33.852 \\
40.856 \\
51.356 \\
68.368\end{array}$ & $\begin{array}{l}-0.02 \\
-0.06 \\
-0.09 \\
-0.10 \\
-0.11 \\
-0.13\end{array}$ & $\begin{array}{l}- \\
- \\
- \\
- \\
- \\
-\end{array}$ & $\begin{array}{l}1696.2 \\
1681.5 \\
1655.3 \\
1632.8 \\
1596.2 \\
1526.0\end{array}$ & & $\begin{array}{l}- \\
- \\
- \\
- \\
-\end{array}$ \\
\hline $\begin{array}{l}423.1 \\
423.1 \\
423.1 \\
\angle 23.1 \\
\angle 23.1 \\
423.1\end{array}$ & $\begin{array}{l}90 \\
70 \\
50 \\
40 \\
30 \\
20\end{array}$ & $\begin{array}{l}21.514 \\
27.165 \\
35.812 \\
44.695 \\
56.753 \\
77.251\end{array}$ & $\begin{array}{l}21.51 ? \\
27.158 \\
36.779 \\
44.653 \\
56.721 \\
77.232\end{array}$ & $\begin{array}{r}0.02 \\
-0.02 \\
-0.09 \\
-0.09 \\
-0.06 \\
-0.02\end{array}$ & $\begin{array}{l}- \\
- \\
- \\
- \\
-\end{array}$ & $\begin{array}{l}1758.1 \\
1744.7 \\
1721.0 \\
1700.7 \\
1667.7 \\
1604.9\end{array}$ & & $\begin{array}{l}- \\
- \\
- \\
- \\
-\end{array}$ \\
\hline
\end{tabular}


Table 4 (Continued)

\begin{tabular}{|c|c|c|c|c|c|c|c|}
\hline $\begin{array}{l}\mathrm{T}, \\
\mathrm{K}\end{array}$ & $\begin{array}{c}\mathrm{V}, \\
\mathrm{cm}^{3} / \mathrm{g}\end{array}$ & $\begin{array}{r}\mathrm{P} \\
\mathrm{exp}, \\
\mathrm{Pa} \times 10^{-5} \\
\end{array}$ & $\begin{array}{c}P_{2 a l^{\prime}} \\
\mathrm{Pa} \times 10^{-5}\end{array}$ & $(\triangle \mathrm{P} / \mathrm{P}) \times 100$ & $\begin{array}{l}\text { axp' } \\
\mathrm{kJ} / \mathrm{kg}\end{array}$ & $\begin{array}{l}\mathrm{h}_{\text {cal }} \\
\mathrm{kJ} / \mathrm{kg}\end{array}$ & $(\Delta \mathrm{h} / \mathrm{h}) \times 100^{\mathrm{a}}$ \\
\hline 473.1 & 90 & 24.470 & 24.486 & 0.06 & - & 1882.6 & - \\
\hline 473.1 & 70 & 31.046 & 31.064 & 0.06 & - & 1871.0 & - \\
\hline 473.1 & 50 & 42.445 & 42.46 .0 & 0.03 & - & 1850.5 & - \\
\hline 473.1 & 40 & 51.970 & 51.976 & 0.01 & - & 1833.1 & - \\
\hline 473.1 & 30 & 66.926 & 66.942 & 0.02 & - & 1804.9 & - \\
\hline 473.1 & 20 & 93.797 & 93.776 & -0.02 & - & 1751.6 & - \\
\hline 523.1 & 90 & 27.368 & 27.420 & 0.19 & - & 2009.7 & - \\
\hline 523.1 & 70 & 34.846 & 34.911 & 0.19 & - & 1999.2 & - \\
\hline 523.1 & 50 & 47.957 & 48.019 & 0.13 & - & 1980.7 & - \\
\hline 523.1 & 40 & 59.032 & 59.104 & 0.12 & - & 1964.9 & - \\
\hline 523.1 & 30 & 76.744 & $76.8 C 5$ & 0.08 & - & 1939.5 & - \\
\hline 523.1 & 20 & 109.614 & 109.486 & -0.12 & - & 1891.7 & - \\
\hline 573.1 & $\exists 0$ & 30.236 & 30.338 & 0.34 & - & 2140.0 & - \\
\hline 573.1 & 70 & 38.585 & 38.731 & 0.38 & - & 2130.5 & - \\
\hline 573.1 & 50 & 53.318 & 53.525 & 0.39 & - & 2113.3 & - \\
\hline 573.1 & 40 & 65.892 & 66.144 & 0.38 & - & 2098.7 & - \\
\hline 573.1 & 30 & 86.218 & 86.506 & 0.33 & - & 2075.1 & - \\
\hline 573.1 & 20 & 124.732 & 124.818 & 0.07 & - & 2030.6 & - \\
\hline
\end{tabular}

${ }^{a}$ Data not jiver. 
Table 5. Results of ammonia F-V-T representation: Comparison with NBS Data ${ }^{7}$

\begin{tabular}{|c|c|c|c|c|c|c|c|}
\hline $\begin{array}{l}\mathrm{T}, \\
\mathrm{K}\end{array}$ & $\mathrm{cm}^{3} ;$ & $\begin{array}{l}\mathrm{P}_{\exp } \\
\mathrm{Pa} \times 10^{-5}\end{array}$ & $\begin{array}{l}\mathrm{P}_{\mathrm{cal}}, \\
\mathrm{Pa} \times 10^{-5}\end{array}$ & $(\Delta \mathrm{P} / \mathrm{P}) \times 100$ & $\begin{array}{l}h_{\text {exp }} \\
k J / k g\end{array}$ & $\begin{array}{l}\mathrm{h}_{\mathrm{cal}}, \\
\mathrm{kJ} / \mathrm{kg}\end{array}$ & $(\Delta h / h) \times 100$ \\
\hline 299.8 & 338.9 & 4.137 & 4.133 & -0.09 & 1512.5 & 1510.5 & -0.13 \\
\hline 299.8 & 160.8 & 8.274 & 8.278 & 0.04 & 1483.7 & 1483.7 & -0.00 \\
\hline 344.2 & 396.5 & 4.137 & 4.131 & -0.14 & 1616.6 & 1613.8 & -0.17 \\
\hline 344.2 & 192.8 & 8.274 & 8.267 & -0.09 & 1600.1 & 1597.4 & -0.16 \\
\hline 344.2 & 124.8 & 12.411 & 12.403 & -0.06 & 1582.9 & 1580.1 & -0.18 \\
\hline 344.2 & 90.6 & 16.548 & 16.538 & -0.06 & 1564.6 & 1561.6 & -0.19 \\
\hline 344.2 & 70.1 & 20.684 & 20.647 & -0.17 & 1545.0 & 1541.8 & -0.21 \\
\hline 388.7 & 451.9 & 4.137 & 4.132 & -0.12 & 1719.4 & 1716.5 & -0.17 \\
\hline 388.7 & 222.1 & 8.274 & 8.268 & -0.06 & 1708.2 & 1705.2 & -0.18 \\
\hline 388.7 & $145: 3$ & 12.411 & 12.415 & 0.03 & 1697.0 & 1693.5 & -0.22 \\
\hline 388.7 & 107.0 & 15.548 & 15.558 & 0.06 & 1685.4 & 1681.4 & -0.24 \\
\hline 388.7 & 84.0 & 20.684 & 20.690 & 0.03 & 1673.6 & 1669.0 & -0.27 \\
\hline 422.0 & 492.7 & 4.137 & 4.132 & -0.12 & 1797.5 & 1794.7 & -0.15 \\
\hline 422.0 & 243.2 & 8.274 & 8.271 & -0.03 & 1788.9 & 1785.5 & -0.19 \\
\hline 422.0 & 159.9 & 12.411 & 12.423 & 0.09 & 1780.0 & 1776.2 & -0.21 \\
\hline 422.0 & 118.3 & 16.548 & 16.574 & 0.16 & 1771.2 & 1766.7 & -0.25 \\
\hline 422.0 & 93.4 & 20.684 & 20.720 & 0.17 & 1762.1 & 1757.0 & -0.28 \\
\hline
\end{tabular}


Table 6. Resulis of isobutane P-V-T representation: Comparison with data of Canjar and Manning ${ }^{8}$

\begin{tabular}{|c|c|c|c|c|c|c|c|}
\hline${ }^{\mathrm{T}} \mathrm{F}$ & $\mathrm{ft}^{3} / \mathrm{lb}$ & $\begin{array}{l}P_{\text {exp' }} \\
\text { psia }\end{array}$ & $\begin{array}{l}P_{\text {cal }}, \\
\text { fsia }\end{array}$ & $(\Delta \mathrm{P} / \mathrm{P}) \times 100$ & $\begin{array}{l}h_{\text {exp }} \\
\text { Btu/1b }\end{array}$ & $\begin{array}{l}h_{c a l}, \\
\text { Btu/lb }\end{array}$ & $(\Delta h / h) \times 100$ \\
\hline 20.00 & 88.360 & 1.000 & 1.000 & -0.03 & 185.50 & 185.53 & 0.01 \\
\hline 20.00 & 5.797 & 14.696 & 14.661 & -0.24 & 183.60 & 183.46 & -0.07 \\
\hline 20.00 & 1.495 & 50.000 & 50.117 & 0.23 & 177.20 & 177.20 & 0.01 \\
\hline 60.00 & 95.740 & 1.000 & 1.000 & 0.02 & 200.30 & 200.49 & 0.09 \\
\hline 60.00 & 6.337 & 14.696 & 14.677 & -0.13 & 199.20 & 198.79 & -0.21 \\
\hline 60.00 & 1.701 & 50.000 & 50.050 & 0.10 & 194.20 & 193.89 & -0.16 \\
\hline 100.00 & 103.100 & 1.000 & 1.001 & 0.07 & 216.60 & 216.53 & -0.03 \\
\hline 100.00 & 6.869 & 14.695 & 14.636 & -0.07 & 215.50 & 215.10 & -0.18 \\
\hline 100.00 & 1.889 & 50.000 & 50.024 & 0.05 & 211.50 & 211.13 & -0.17 \\
\hline 100.00 & 0.837 & 100.000 & 100.132 & 0.13 & 204.70 & 204.40 & -0.14 \\
\hline 140.00 & 110.500 & 1.000 & 1.001 & 0.07 & 233.90 & 233.61 & -0.12 \\
\hline 140.00 & 7.396 & 14.695 & 14.589 & -0.05 & 232.80 & 232.41 & -0.17 \\
\hline 140.00 & 2.066 & 50.000 & 50.020 & 0.04 & 229.40 & 229.11 & -0.13 \\
\hline 140.00 & 0.948 & 100.001 & 100.109 & 0.11 & 224.10 & 223.81 & -0.13 \\
\hline 140.00 & 0.564 & 150.001 & 150.170 & 0.11 & 217.60 & 217.35 & -0.12 \\
\hline 180.00 & 117.800 & 1.000 & 1.302 & 0.16 & 251.90 & 251.71 & -0.07 \\
\hline 180.00 & 7.919 & 14.695 & 14.589 & -0.05 & 250.90 & 250.68 & -0.09 \\
\hline 180.00 & 2.237 & 50.000 & 50.1000 & 0.00 & 248.10 & 247.90 & -0.08 \\
\hline 180.00 & 1.048 & 100.000 & 100.133 & 0.13 & 243.70 & 243.56 & -0.06 \\
\hline 180.00 & 0.647 & 150.000 & 150.177 & 0.12 & 238.70 & 238.62 & -0.03 \\
\hline 180.00 & 0.439 & 200.000 & 200.190 & 0.10 & 232.80 & 232.69 & -0.05 \\
\hline 180.00 & 0.393 & 250.000 & 249.798 & -0.08 & 225.00 & 224.76 & -0.11 \\
\hline
\end{tabular}


Table 6 (Continued)

\begin{tabular}{|c|c|c|c|c|c|c|c|}
\hline${ }^{\mathrm{T}} \mathrm{F}$ & $\begin{array}{c}v, \\
\mathrm{ft}^{3 / 1 b}\end{array}$ & $\begin{array}{l}\mathrm{P}_{\text {exp' }}, \\
\text { psia }\end{array}$ & $\begin{array}{l}\mathrm{P}_{\text {cal }}, \\
\text { psia }\end{array}$ & $(\Delta \mathrm{P} / \mathrm{P}) \times 100$ & $\begin{array}{l}h_{\text {exp }} \\
\text { Btu/1b }\end{array}$ & $\begin{array}{l}\mathrm{h}_{\mathrm{cal}}, \\
\mathrm{Btu} / \mathrm{lb}\end{array}$ & $(\Delta h / h) \times 100$ \\
\hline 220.00 & 125.300 & 1.000 & 1.001 & 0.07 & 270.90 & 270.80 & -0.04 \\
\hline 220.00 & 8.438 & 14.696 & 14.690 & -0.04 & 269.90 & 269.91 & 0.00 \\
\hline 220.00 & 2.402 & 50.000 & 50.007 & 0.01 & 267.60 & 267.52 & -0.03 \\
\hline 220.00 & 1.143 & 100.000 & 100.033 & 0.03 & 263.80 & 263.90 & 0.04 \\
\hline 220.00 & 0.719 & 150.000 & 150.158 & 0.11 & 259.80 & 259.90 & 0.04 \\
\hline 220.00 & 0.504 & 200.000 & 200.246 & 0.12 & 255.30 & 255.40 & 0.04 \\
\hline 220.00 & 0.369 & 250.000 & 251.580 & 0.63 & 250.10 & 250.01 & -0.04 \\
\hline 220.00 & 0.277 & 300.000 & 299.952 & -0.02 & 243.60 & 243.64 & 0.02 \\
\hline 220.00 & 0.208 & 350.000 & 342.807 & -2.06 & 234.10 & 235.52 & 0.60 \\
\hline 240.00 & 129.100 & 1.000 & 1.000 & -0.01 & 280.70 & 280.71 & 0.00 \\
\hline 240.00 & 8.696 & 14.696 & 14.690 & -0.04 & 279.80 & 279.88 & 0.03 \\
\hline 240.00 & 2.484 & 50.000 & 49.994 & -0.01 & 277.60 & 277.66 & 0.02 \\
\hline 240.00 & 1.188 & 100.000 & 100.046 & 0.05 & 274.10 & 274.31 & 0.08 \\
\hline 240.00 & 0.753 & 150.000 & 150.138 & 0.09 & 270.50 & 270.67 & 0.06 \\
\hline 240.00 & 0.534 & 200.000 & 200.271 & 0.14 & 266.30 & 266.66 & 0.13 \\
\hline 240.00 & 0.399 & 250.000 & 250.305 & 0.12 & 261.80 & 262.13 & 0.13 \\
\hline 240.00 & 0.306 & 300.000 & 300.245 & 0.08 & 256.50 & 256.82 & 0.12 \\
\hline 240.00 & 0.235 & 350.000 & 349.818 & -0.05 & 249.90 & 250.17 & 0.11 \\
\hline 240.00 & 0.170 & 400.000 & 398.30 .5 & -0.42 & 238.30 & 240.06 & 0.74 \\
\hline 260.00 & 123.800 & 1.000 & 1.000 & -0.01 & 290.80 & 290.85 & 0.02 \\
\hline 260.00 & 8.953 & 14.696 & 14.691 & -0.03 & 290.00 & 290.07 & 0.03 \\
\hline 260.00 & 2.564 & 50.000 & 50.005 & 0.01 & 287.80 & 288.00 & 0.07 \\
\hline 260.00 & 1.232 & 100.000 & 100.065 & 0.07 & 284.60 & 284.90 & 0.11 \\
\hline 260.00 & 0.786 & 150.000 & 150.094 & 0.06 & 281.10 & 281.57 & 0.17 \\
\hline 260.00 & 0.562 & 200.000 & 200.159 & 0.08 & 277.40 & 277.95 & 0.20 \\
\hline 260.00 & 0.425 & 250.000 & 250.210 & 0.08 & 273.30 & 273.96 & 0.24 \\
\hline
\end{tabular}


Table 6 (Continued)

\begin{tabular}{|c|c|c|c|c|c|c|c|}
\hline${ }^{\mathrm{T}} \mathrm{F}$ & $\begin{array}{c}\mathrm{v}, \\
\mathrm{ft}\end{array}$ & $\begin{array}{l}\mathrm{P}_{\text {exp' }} \\
\text { psia }\end{array}$ & $\begin{array}{l}P_{\text {cal }} \text { ' } \\
\text { psia }\end{array}$ & $(\Delta \mathrm{P} / \mathrm{P}) \times 100$ & $\begin{array}{l}h_{\text {exp }} \\
\text { Btu!' } 1 b\end{array}$ & $\begin{array}{l}\mathrm{h}_{\text {cal }}, \\
\text { Btu/lb }\end{array}$ & $(\Delta h / h) \times 100$ \\
\hline 260.00 & 0.331 & 300.000 & 300.162 & 0.05 & 268.70 & 269.45 & 0.28 \\
\hline 260.00 & 0.262 & 350.000 & 350.000 & -0.00 & 263.30 & 264.17 & 0.33 \\
\hline 260.00 & 0.206 & 400.000 & 399.385 & -0.15 & 256.80 & 257.62 & 0.32 \\
\hline 260.00 & 0.185 & 420.000 & 419.315 & -0.16 & 253.90 & 254.28 & 0.15 \\
\hline 260.00 & 0.164 & 440.000 & 438.757 & -0.28 & 249.60 & 250.29 & 0.28 \\
\hline 260.00 & 0.142 & 460.000 & 457.770 & -0.48 & 244.50 & 244.92 & 0.17 \\
\hline 260.00 & 0.115 & 480.000 & 472.690 & -1.52 & 237.30 & 236.29 & -0.73 \\
\hline 280.00 & 136.500 & 1.000 & 1.000 & -0.01 & 301.20 & 301.23 & 0) 1$. \\
\hline 280.00 & 9.209 & 14.696 & 14.693 & -0.02 & 300.40 & 300.50 & 0.33 \\
\hline 280.00 & 2.645 & 50.000 & 49.986 & -0.03 & 298.40 & 298.56 & 0.36 \\
\hline 280.00 & 1.276 & 100.000 & 100.029 & 0.03 & 295.30 & 295.68 & 0.13 \\
\hline 280.00 & 0.819 & 150.000 & 150.045 & 0.03 & 292.10 & 292.62 & 0.18 \\
\hline 280.00 & 0.588 & 200.000 & 200.048 & 0.02 & 288.60 & 289.32 & 0.25 \\
\hline 280.00 & 0.449 & 250.000 & 250.091 & 0.04 & 284.90 & 285.75 & 0.30 \\
\hline 280.00 & 0.354 & 300.000 & 299.958 & -0.01 & 289.90 & 281.82 & 0.33 \\
\hline 280.00 & 0.285 & 350.000 & 349.855 & -0.04 & 276.30 & 277.39 & 0.39 \\
\hline 280.00 & 0.231 & 400.000 & 399.730 & -0.07 & 271.10 & 272.25 & 0.42 \\
\hline 280.00 & 0.212 & 420.000 & 419.999 & -0.00 & 268.70 & 269.86 & 0.43 \\
\hline 280.00 & 0.194 & 440.000 & 439.831 & -0.04 & 266.10 & 267.29 & 0.45 \\
\hline 280.00 & 0.177 & 460.000 & 459.725 & -0.06 & 263.20 & 264.40 & 0.46 \\
\hline 280.00 & 0.160 & 480.000 & 479.594 & -0.08 & 259.90 & 261.08 & 0.46 \\
\hline 280.00 & 0.144 & 500.000 & 499.039 & -0.19 & 256.10 & 257.19 & 0.42 \\
\hline 280.00 & 0.127 & 520.000 & 518.045 & -0.38 & 251.30 & 252.28 & 0.39 \\
\hline 280.00 & 0.107 & 540.000 & 536.400 & -0.67 & 244.20 & 245.09 & 0.37 \\
\hline 280.00 & 0.075 & 560.000 & 553.444 & -1.17 & 228.30 & 228.79 & 0.21 \\
\hline 280.00 & 0.057 & 580.000 & 570.253 & -1.68 & 214.20 & 214.32 & 0.05 \\
\hline 280.00 & 0.053 & 600.000 & 589.137 & -1.81 & 209.40 & 209.50 & 0.05 \\
\hline
\end{tabular}


Table 6 (Continued)

\begin{tabular}{|c|c|c|c|c|c|c|c|}
\hline$\stackrel{\mathrm{T}}{{ }^{\circ} \mathrm{F}}$ & $\begin{array}{c}v \\
\mathrm{ft}^{3} / 1 \mathrm{~b}\end{array}$ & $\begin{array}{l}\text { Pexp' } \\
\text { psia }\end{array}$ & $\begin{array}{l}P_{\text {cal }}, \\
\text { psia }\end{array}$ & $(\Delta \mathrm{P} / \mathrm{P}) \times 100$ & $\begin{array}{l}\mathrm{h}_{\mathrm{exp}} \\
\text { Btu/1b}\end{array}$ & $\begin{array}{l}\mathrm{h}_{\mathrm{cal}}, \\
\text { Btu/1b }\end{array}$ & $(\Delta h / h) \times 100$ \\
\hline 230.00 & 0.048 & 640.000 & 631.028 & -1.40 & 204.80 & 204.71 & -0.04 \\
\hline 230.00 & 0.046 & 680.000 & 676.788 & -0.47 & 202.50 & 201.83 & -0.33 \\
\hline 230.00 & 0.046 & 700.000 & 694.988 & -0.72 & 201.70 & 200.96 & -0.36 \\
\hline 230.00 & 0.043 & 800.000 & 789.680 & -1.29 & 198.70 & 197.70 & -0.50 \\
\hline 290.00 & 0.042 & 900.000 & 884.589 & -1.71 & 196.70 & 195.54 & -0.59 \\
\hline 290.00 & 0.041 & $1,000.000$ & 979.759 & -2.02 & 195.30 & 193.94 & -0.70 \\
\hline 280.00 & 0.039 & $1,200.000$ & $1,281.724$ & 6.81 & 191.30 & 190.75 & -0.29 \\
\hline 280.00 & 0.038 & $1,400.000$ & $1,405.846$ & 0.42 & 191.00 & 189.93 & -0.56 \\
\hline 280.00 & 0.037 & $1,600.000$ & $1,654.601$ & 3.41 & 190.80 & 188.76 & -1.07 \\
\hline 280.00 & 0.037 & 1.800 .000 & $1,762.981$ & -2.06 & 190.50 & 188.40 & -1.10 \\
\hline 280.00 & 0.036 & $2,000.000$ & $1,936.128$ & -3.19 & 190.40 & 187.98 & -1.27 \\
\hline 280.00 & 0.035 & $2,500.000$ & $2,452.152$ & -1.91 & 190.00 & 187.49 & -1.32 \\
\hline 280.00 & 0.034 & $3,000.000$ & $2,989.599$ & -0.35 & 190.20 & 187.77 & -1.28 \\
\hline 280.00 & 0.033 & $3,500.000$ & $3,566.762$ & 1.91 & 190.70 & 188.65 & -1.08 \\
\hline 320.00 & 143.900 & 1.000 & 1.000 & -0.01 & 322.70 & 322.67 & -0.01 \\
\hline 320.00 & 9.724 & 14.696 & 14.690 & -0.04 & 322.00 & 322.02 & 0.01 \\
\hline 320.00 & 2.804 & 50.000 & 49.975 & -0.05 & $320: 20$ & 320.32 & 0.04 \\
\hline 320.00 & 1.362 & 100.000 & 99.970 & -0.03 & 317.70 & 317.81 & 0.03 \\
\hline 320.00 & 0.881 & 150.000 & 149.952 & -0.03 & 315.00 & 315.17 & 0.05 \\
\hline 320.00 & 0.639 & 200.000 & 199.917 & -0.04 & 312.20 & 312.38 & 0.06 \\
\hline 320.00 & 0.493 & 250.000 & 249.879 & -0.05 & 309.30 & 309.43 & 0.04 \\
\hline 320.00 & 0.395 & 300.000 & 299.833 & -0.06 & 306.10 & 306.27 & 0.06 \\
\hline$\$ 20.00$ & 0.325 & 350.000 & 349.608 & -0.11 & 302.70 & 302.89 & 0.06 \\
\hline$\$ 20.00$ & 0.271 & 400.000 & 399.435 & -0.14 & 299.10 & 299.19 & 0.03 \\
\hline$\$ 20.00$ & 0.193 & 500.000 & 498.742 & -0.25 & 290.50 & 290.57 & 0.02 \\
\hline 320.00 & 0.136 & 600.000 & 597.677 & -0.39 & 279.30 & 279.17 & -0.05 \\
\hline 520.00 & 0.091 & 700.000 & 698.256 & -0.25 & 262.70 & 262.43 & -0.10 \\
\hline
\end{tabular}


Table 6 (Continued)

\begin{tabular}{|c|c|c|c|c|c|c|c|}
\hline${ }^{\mathrm{T}} \mathrm{F}$ & $\begin{array}{c}v \\
\mathrm{ft}^{3} / 1 \mathrm{~b}\end{array}$ & $\begin{array}{l}P_{\text {exp }} \\
\text { psia }\end{array}$ & $\begin{array}{l}P_{\text {cal }}, \\
\text { psia }\end{array}$ & $(\Delta P / P) \times 100$ & $\begin{array}{l}h_{\text {exp }} \\
\text { Btu; } 1 b\end{array}$ & $\begin{array}{l}\mathrm{h}_{\mathrm{cal}}, \\
\text { Btu/lb }\end{array}$ & $(\Delta h / h) \times 100$ \\
\hline 320.00 & 0.065 & 800.000 & 797.061 & -0.37 & 247.20 & 246.40 & -0.32 \\
\hline 320.00 & 0.055 & 900.000 & 897.522 & -0.28 & 238.10 & 236.99 & -0.47 \\
\hline 320.00 & 0.050 & $1,000.000$ & 999.437 & -0.06 & 232.90 & 231.69 & $-0.5 ?$ \\
\hline 320.00 & 0.045 & $1,200.000$ & $1,195.972$ & -0.34 & 227.30 & 226.12 & $-0.5 ?$ \\
\hline 320.00 & 0.043 & $1,400.000$ & $1,396.233$ & -0.27 & 224.20 & 222.92 & -0.57 \\
\hline 320.00 & 0.041 & $1,600.000$ & $1,599.795$ & -0.01 & 222.20 & 220.80 & -0.6 .3 \\
\hline 320.00 & 0.040 & $1,800.000$ & $1,799.342$ & -0.04 & 220.90 & 219.36 & $-0.7)$ \\
\hline 320.00 & 0.039 & $2,000.000$ & $1,995.863$ & -0.21 & 219.90 & 218.33 & $-0.7 \mathrm{I}$ \\
\hline 320.00 & 0.038 & $2,500.000$ & $2,488.882$ & -0.44 & 218.70 & 216.83 & $-0.8 \bar{j}$ \\
\hline 320.00 & 0.036 & $3,000.000$ & $2,995.689$ & -0.14 & 218.30 & 216.27 & -0.93 \\
\hline 320.00 & 0.035 & $3,500.000$ & $3,511.837$ & 0.34 & 218.20 & 216.32 & $-0.8 \overline{5}$ \\
\hline 400.00 & 158.600 & 1.000 & 1.000 & 0.04 & 368.30 & 368.21 & -0.03 \\
\hline 400.00 & 10.740 & 14.696 & 14.697 & 0.01 & 367.80 & 367.69 & -0.03 \\
\hline 400.00 & 3.115 & 50.000 & 49.991 & -0.02 & 366.50 & 366.33 & -0.05 \\
\hline 400.00 & $1.52 ?$ & 100.000 & 99.972 & -0.03 & 364.50 & 364.35 & -0.04 \\
\hline 400.00 & 0.997 & 150.000 & 150.008 & 0.01 & 362.50 & 362.30 & -0.05 \\
\hline 400.00 & 0.732 & 200.000 & 199.995 & -0.00 & 360.50 & 360.19 & -0.08 \\
\hline 400.00 & 0.572 & 250.000 & 250.083 & 0.03 & 358.30 & 358.01 & -0.08 \\
\hline 400.00 & 0.466 & 300.000 & 300.123 & 0.04 & 356.10 & 355.74 & -0.10 \\
\hline 400.00 & 0.389 & 350.000 & 351. 123 & 0.04 & 353.80 & 353.39 & -0.12 \\
\hline 400.00 & 0.332 & 400.000 & 401.177 & 0.04 & 351.40 & 350.94 & $-0.1 j$ \\
\hline 400.00 & 0.251 & 500.000 & 500.295 & 0.06 & 346.20 & 345.72 & -0.14 \\
\hline 400.00 & 0.197 & 600.000 & 600.194 & 0.03 & 340.60 & 340.04 & $-0.1^{7}$ \\
\hline 400.00 & 0.158 & 700.000 & 701.207 & 0.03 & 334.50 & 333.83 & -0.20 \\
\hline 400.00 & 0.129 & 800.000 & 800.135 & 0.02 & 327.90 & 327.13 & -0.23 \\
\hline 400.00 & 0.107 & 900.000 & 899.763 & -0.03 & 320.90 & 320.17 & -0.23 \\
\hline 400.00 & 0.091 & $1,000.000$ & $998.42 J$ & -0.16 & 314.00 & 313.43 & -0.18 \\
\hline 400.00 & 0.071 & $1,200.000$ & $1,196.562$ & -0.29 & 303.20 & 310.98 & -0.40 \\
\hline
\end{tabular}


Table 6 (Continued)

\begin{tabular}{|c|c|c|c|c|c|c|c|}
\hline${ }^{\mathrm{T}} \mathrm{F}$ & $\begin{array}{c}\mathrm{v} \\
\mathrm{ft}\end{array}$ & $\begin{array}{l}P_{\text {exp }}, \\
\text { psia }\end{array}$ & $\begin{array}{l}P_{\text {cal }}, \\
\text { psia }\end{array}$ & $(\Delta \mathrm{P} / \mathrm{P}) \times 100$ & $\begin{array}{l}h_{\exp }, \\
\text { Btu/lb }\end{array}$ & $\begin{array}{l}\mathrm{h}_{\text {cal }}, \\
\text { Btu/lb }\end{array}$ & $(\Delta \mathrm{h} / \mathrm{h}) \times 100$ \\
\hline 400.00 & 0.060 & $1,400.000$ & $1,404.932$ & 0.35 & 294.10 & 293.53 & -0.20 \\
\hline 400.00 & 0.053 & $1,500.000$ & $1,618.458$ & 1.15 & 288.60 & 287.69 & -0.32 \\
\hline 400.00 & 0.050 & $1,300.000$ & $1,805.376$ & 0.30 & 284.60 & 284.13 & -0.17 \\
\hline 400.00 & 0.047 & $2,000.000$ & $2,004.644$ & 0.23 & 281.80 & 281.38 & -0.15 \\
\hline 400.00 & 0.043 & $2,500.000$ & $2,500.424$ & 0.02 & 277.50 & 277.29 & -0.08 \\
\hline 400.00 & 0.040 & $3,000.000$ & $3,000.752$ & 0.03 & 275.40 & 275.29 & -0.40 \\
\hline 400.00 & 0.039 & $3,500.000$ & $3,502.994$ & 0.09 & 274.40 & 274.40 & 0.00 \\
\hline 6100.00 & 195.600 & 1.000 & 1.000 & 0.01 & 496.90 & 496.32 & -0.12 \\
\hline 600.00 & 13.280 & 14.696 & 14.695 & -0.01 & 496.50 & 495.99 & -0.10 \\
\hline 630.00 & 3.880 & 50.000 & 49.981 & -0.04 & 495.60 & 495.12 & -0.10 \\
\hline 630.00 & 1.923 & 100.000 & 99.952 & -0.05 & 494.40 & 493.87 & -0.11 \\
\hline 630.00 & 1.271 & 150.000 & 149.885 & -0.08 & 493.10 & 492.61 & -0.10 \\
\hline 630.00 & 0.945 & 200.000 & 199.905 & -0.05 & 491.90 & 491.34 & -0.11 \\
\hline 630.00 & 0.745 & 250.000 & 249.912 & -0.04 & 490.60 & 490.05 & -0.11 \\
\hline 630.00 & 0.618 & 300.000 & 299.928 & -0.02 & 489.30 . & 488.75 & -0.11 \\
\hline 600.00 & 0.525 & 350.000 & 349.904 & -0.03 & $488.00^{\circ}$ & 487.44 & -0.11 \\
\hline 500.00 & 0.456 & 400.000 & 399.965 & -0.01 & 486.70 & 486.12 & -0.12 \\
\hline 600.00 & 0.358 & 500.000 & 500.068 & 0.01 & 484.00 & 483.44 & -0.12 \\
\hline 600.00 & 0.293 & 600.000 & 600.425 & 0.07 & 481.30 & 480.72 & -0.12 \\
\hline 600.00 & 0.247 & 700.000 & 700.804 & 0.11 & 478.60 & 477.97 & -0.13 \\
\hline 600.00 & 0.213 & 800.000 & 801.107 & 0.14 & 475.90 & 475.21 & -0.14 \\
\hline 600.00 & 0.187 & 900.000 & 899.971 & -0.00 & 473.10 & 472.49 & -0.13 \\
\hline 600.00 & 0.166 & $1,000.000$ & 998.270 & -0.17 & 470.30 & 469.80 & -0.11 \\
\hline 600.00 & 0.136 & $1,200.000$ & $1,195.681$ & -0.36 & 464.80 & 464.51 & -0.06 \\
\hline 600.00 & 0.114 & $1,400.000$ & $1,401.517$ & 0.11 & 459.70 & 459.26 & -0.10 \\
\hline 600.00 & 0.098 & $1,600.000$ & $1,606.845$ & 0.43 & 454.90 & 454.42 & -0.10 \\
\hline$\epsilon 00.00$ & 0.088 & 1.800 .000 & $1,796.416$ & -0.20 & 450.50 & 450.38 & -0.03 \\
\hline$\epsilon 00.00$ & 0.079 & $2,000.000$ & $2,006.873$ & 0.34 & 447.10 & 446.40 & -0.16 \\
\hline
\end{tabular}


Table 6 (Continued)

\begin{tabular}{|c|c|c|c|c|c|c|c|}
\hline$\stackrel{\mathrm{T}}{{ }^{\circ} \mathrm{F}}$ & $\mathrm{ft}^{\mathrm{v}} / \mathrm{lb}$ & $\begin{array}{l}\text { Pexp' } \\
\text { psia }\end{array}$ & $\begin{array}{l}P_{\text {cal }} \\
\text { psia }\end{array}$ & $(\triangle P / P) \times 100$ & $\begin{array}{l}\mathrm{h}_{\mathrm{exp}}, \\
\text { Btu, lb }\end{array}$ & $\begin{array}{l}\mathrm{h}_{\mathrm{cal}}, \\
\text { Btu/1b }\end{array}$ & $(\Delta \mathrm{h} / \mathrm{h}) \times 100$ \\
\hline 600.00 & 0.065 & $2,500.000$ & $2,512.595$ & 0.51 & 439.10 & 438.83 & -0.05 \\
\hline 600.00 & 0.058 & $3,000.000$ & $2,995.595$ & -0.15 & 433.70 & 433.71 & 0.00 \\
\hline 600.00 & 0.053 & $3,500.000$ & $3,478.769$ & -0.61 & 430.00 & 430.02 & 0.01 \\
\hline 900.00 & 251.000 & 1.000 & 1.000 & 0.01 & 721.80 & 721.41 & -0.05 \\
\hline 900.00 & 17.080 & 14.696 & 14.686 & -0.07 & 721.60 & 721.20 & -0.05 \\
\hline 900.00 & 5.009 & 50.000 & 49.978 & -0.04 & 721.00 & 720.66 & -0.05 \\
\hline 900.00 & 2.499 & 100.000 & 99.945 & -0.05 & 720.30 & 719.90 & -0.05 \\
\hline 900.00 & 1.661 & 150.000 & 149.919 & -0.05 & 719.60 & 719.13 & -0.05 \\
\hline 900.00 & 1.243 & 200.000 & 199.837 & -0.08 & 718.80 & 718.37 & -0.06 \\
\hline 900.00 & 0.992 & 250.000 & 249.882 & -0.05 & 718.10 & 717.62 & -0.07 \\
\hline 900.00 & 0.825 & 300.050 & 299.377 & -0.04 & 717.40 & 716.86 & -0.03 \\
\hline 900.00 & 0.705 & 350.030 & 349.858 & -0.04 & 716.70 & 716.11 & -0.03 \\
\hline 900.00 & 0.616 & 400.030 & 399.341 & -0.04 & 715.90 & 715.35 & -0.03 \\
\hline 900.00 & 0.491 & 500.030 & 499.839 & -0.03 & 714.50 & 713.86 & -0.07 \\
\hline 900.00 & 0.408 & 600.030 & 599.324 & -0.03 & 713.10 & 712.39 & -0.13 \\
\hline 900.00 & 0.349 & 700.030 & 699.674 & -0.05 & 711.60 & 710.94 & -0.07 \\
\hline 900.00 & 0.304 & 800.030 & 799.848 & -0.02 & 710.20 & 709.50 & -0.13 \\
\hline 900.00 & 0.270 & 900.030 & 900.048 & 0.01 & 708.90 & 708.09 & -0.11 \\
\hline 900.00 & 0.243 & $1,000.030$ & $1,000.263$ & 0.03 & 707.50 & 706.70 & -0.11 \\
\hline 900.00 & 0.202 & $1,200.050$ & $1,200.067$ & 0.01 & 704.90 & 704.02 & -0.13 \\
\hline 900.00 & 0.174 & $1,400.030$ & $1,400.008$ & 0.00 & 702.40 & 701.46 & -0.13 \\
\hline 900.00 & 0.152 & $1,600.030$ & $1,600.411$ & 0.03 & 700.00 & 699.03 & -0.17 \\
\hline 900.00 & 0.136 & $1,800.030$ & $1,801.689$ & 0.09 & 697.70 & 696.73 & -0.17 \\
\hline 900.00 & 0.123 & $2,000.030$ & $2,000.834$ & 0.04 & 695.60 & 694.60 & -0.17 \\
\hline 900.00 & 0.101 & $2,500.030$ & $2,498.969$ & -0.04 & 690.90 & 689.97 & -0.15 \\
\hline 900.00 & 0.087 & $3,000.030$ & $2,994.495$ & -0.18 & 687.00 & 685.99 & -0.15 \\
\hline 900.00 & 0.077 & $3,500.000$ & $3,487.930$ & -0.34 & 683.80 & 682.85 & -0.17 \\
\hline
\end{tabular}


The relatively large discrepancies exhibited near the critical region are due partly to the condition that, in the least-squares algorithm, the analytical model is forced to reproduce the critical point exactly $\left[\mathrm{P}_{\mathrm{r}}=1\right.$ and $\left(\partial \mathrm{P}{ }_{\mathrm{r}} / \partial \mathrm{v}_{\mathrm{r}}\right)=\left(\partial^{2} \mathrm{P}_{\mathrm{r}} / \partial \mathrm{v}_{\mathrm{r}}^{2}\right) \equiv 0$ at $\left.\mathrm{T}_{\mathrm{r}}=\mathrm{v}_{\mathrm{r}}=1\right]$. By relaxing these constraints, the errors would be reduced but the selfconsistent nature of the representation would be lost.

\subsection{Vapor Pressure and Saturated Liquid Volume}

The vapor pressure and saturated liquid volume $\left(\rho_{\ell}^{-1}\right)$ correlations are compared with the data in Tables 7 and 8 for temperatures up to the critical value. For ammonia, the errors in both vapor pressure and specific volume are negligible. In the case of isobutane, the magnitude of the error is on the order of a tenth of a percent which is satisfactory for most applications. 
Tajle 7. Results of the vapor pressure and saturated liquid volume representation for ammonia: Comparison with data of Davies ${ }^{5}$

\begin{tabular}{|c|c|c|c|c|c|c|}
\hline $\begin{array}{l}\mathrm{T}, \\
\mathrm{K}\end{array}$ & $\begin{array}{l}P_{\text {exp }}, \\
\text { atm }\end{array}$ & $\begin{array}{l}P_{\text {cal }}, \\
\text { atm }\end{array}$ & $(\Delta \mathrm{P} / \mathrm{P}) \times 100$ & $\begin{array}{c}v_{\exp } \\
\mathrm{cm}^{3 / \mathrm{mol}}\end{array}$ & $\begin{array}{c}v_{c a 1}, \\
\mathrm{~cm}^{3} / \mathrm{mol}\end{array}$ & $(\Delta v / v) \times 100$ \\
\hline 200 & 0.08553 & 0.08557 & 0.05 & 23.37 & 23.36 & -0.04 \\
\hline .210 & 0.1756 & 0.1757 & 0.06 & 2.3 .74 & 23.74 & 0.00 \\
\hline 220 & 0.3347 & 0.3349 & 0.06 & 24.13 & 24.13 & 0.00 \\
\hline 230 & 0.5982 & 0.5986 & 0.07 & 24.55 & 24.55 & 0.00 \\
\hline 240 & 1.0124 & 1.0126 & 0.02 & 27.99 & 24.99 & 0.00 \\
\hline 250 & 1.632 & 1.633 & 0.06 & 25.46 & 25.46 & 0.00 \\
\hline 260 & 2.526 & 2.526 & 0.00 & 25.96 & 25.96 & 0.00 \\
\hline 270 & 3.769 & 3.769 & 0.00 & 25.50 & 26.50 & 0.00 \\
\hline 280 & 5.446 & 5.446 & 0.00 & 27.07 & 27.08 & 0.04 \\
\hline 290 & 7.652 & 7.651 & -0.01 & 27.70 & 27.70 & 0.00 \\
\hline 300 & 10.485 & 10.488 & 0.03 & 23.39 & 28.39 & 0.00 \\
\hline 310 & 14.063 & 14.064 & 0.01 & 27.14 & 29.14 & 0.00 \\
\hline 320 & 18.49 & 18.49 & 0.00 & 29.98 & 29.98 & 0.00 \\
\hline 330 & 23.90 & 23.91 & 0.04 & 33.92 & 30.92 & 0.00 \\
\hline 340 & 30.42 & 30.43 & 0.03 & 31.99 & 31.99 & 0.00 \\
\hline 350 & 38.19 & 38.20 & 0.03 & 33.24 & 33.25 & 0.03 \\
\hline 360 & 47.40 & 47.37 & -0.06 & 37.73 & 34.74 & 0.03 \\
\hline 370 & 58.14 & 58.09 & -0.09 & 35.58 & 36.60 & 0.05 \\
\hline 380 & 70.60 & 70.54 & -0.08 & 39.03 & 39.05 & 0.05 \\
\hline 390 & 84.94 & 84.90 & -0.05 & 42.61 & 42.63 & 0.05 \\
\hline .100 & 101.5 & $1 \mathrm{Cl} 1.4$ & -0.10 & $4 \ni .45$ & 49.50 & 0.10 \\
\hline 405.44 & 111.3 & 111.3 & 0 & 72.33 & 72.33 & 0 \\
\hline
\end{tabular}


Table 8. Results of the vapor pressure and saturated liquid volume representation for isobutane: Comparison with the data of Canjar and Manning ${ }^{8}$

\begin{tabular}{|c|c|c|c|c|c|c|}
\hline${ }^{\mathrm{T}} \mathrm{F}$ & $\begin{array}{l}\text { Pexp' } \\
\text { psia }\end{array}$ & $\begin{array}{l}P_{\text {cal }} \\
\text { psia }\end{array}$ & $(\Delta \mathrm{P} / \mathrm{P}) \times 100$ & $\begin{array}{l}v_{\exp } \\
\mathrm{ft}^{3} / 1 \mathrm{~b}\end{array}$ & $\begin{array}{l}v_{\mathrm{cal}}, \\
\mathrm{ft} 3 / 1 \mathrm{~b}\end{array}$ & $(\Delta v / v) \times 100$ \\
\hline 0.0 & 11.530 & 11.552 & 0.19 & 0.02668 & 0.02667 & -0.05 \\
\hline 10.00 & 14.414 & 14.420 & 0.04 & 0.02695 & 0.02695 & 0.00 \\
\hline 10.89 & 14.696 & 1.4 .700 & 0.03 & 0.02699 & 0.02698 & -0.05 \\
\hline 20.00 & 17.823 & 17.816 & -0.04 & 0.02723 & 0.02724 & 0.05 \\
\hline 30.00 & 21.829 & 21.803 & -0.12 & 0.02753 & 0.02754 & 0.05 \\
\hline 40.00 & 26.482 & 26.447 & -0.13 & 0.02783 & 0.02786 & 0.09 \\
\hline 50.00 & 31.861 & 31.816 & -0.14 & 0.02815 & 0.02818 & 0.10 \\
\hline 60.00 & 38.036 & 37.979 & -0.15 & 0.02848 & 0.02851 & 0.12 \\
\hline 70.00 & 45.059 & 45.011 & -0.11 & 0.02882 & 0.02886 & 0.14 \\
\hline 80.00 & 52.975 & 52.987 & 0.02 & 0.02918 & 0.02922 & 0.15 \\
\hline 90.00 & 61.953 & 61.984 & 0.05 & 0.02956 & 0.02960 & 0.14 \\
\hline 100.00 & 72.037 & 72.082 & 0.06 & 0.02996 & 0.03000 & 0.13 \\
\hline 110.00 & 83.218 & 83.365 & 0.18 & 0.03039 & 0.03042 & 0.09 \\
\hline 120.00 & 95.733 & 95.917 & 0.19 & 0.03084 & 0.03086 & 0.07 \\
\hline 130.00 & 109.72 & 109.83 & 0.10 & 0.03131 & 0.03133 & 0.07 \\
\hline 140.00 & 125.18 & 1.25 .18 & 0.00 . & 0.03182 & 0.03183 & 0.04 \\
\hline 150.00 & 141.98 & 142.08 & 0.07 & 0.03237 & 0.03237 & 0.00 \\
\hline 160.00 & 160.55 & 160.62 & 0.04 & 0.03295 & 0.03295 & 0.01 \\
\hline 170.00 & 180.89 & 180.90 & 0.01 & 0.03358 & 0.03359 & 0.02 \\
\hline 180.00 & 203.04 & 203.03 & -0.00 & 0.03428 & 0.03428 & 0.01 \\
\hline
\end{tabular}


Table 8 (Continued)

\begin{tabular}{|c|c|c|c|c|c|c|}
\hline${ }^{\mathrm{T}} \mathrm{F}$ & $\begin{array}{l}\overline{\exp } \\
\text { Isia }\end{array}$ & $\begin{array}{l}\mathrm{P}_{\text {cal }}, \\
\text { psia }\end{array}$ & $(\Delta P / P) \times 100$ & $\begin{array}{r}v_{\exp } \\
f t^{3} / 1 b\end{array}$ & $\begin{array}{c}v_{\mathrm{cal}}, \\
\mathrm{ft}{ }^{3} / 1 \mathrm{~b}\end{array}$ & $(\Delta v / v) \times 10($ \\
\hline 190.00 & 227.20 & 227.12 & -0.04 & 0.03507 & 0.03506 & -0.04 \\
\hline 200.00 & 253.41 & 253.28 & -0.05 & 0.03593 & 0.03593 & -0.00 \\
\hline 210.00 & 231.85 & 281.64 & -0.07 & 0.03691 & 0.03693 & 0.05 \\
\hline 220.00 & 312.60 & 312.33 & -0.09 & 0.03810 & 0.03810 & -0.01 \\
\hline 230.00 & 345.82 & 345.48 & -0.10 & 0.03950 & 0.03950 & 0.01 \\
\hline 240.00 & $3: 1.57$ & 381.24 & -0.09 & 0.04120 & 0.04127 & 0.16 \\
\hline 250.00 & $4 \doteq 0.11$ & 419.76 & -0.08 & C. 04360 & 0.04362 & 0.03 \\
\hline $2 \epsilon .0 .00$ & 451.44 & 461.21 & -0.05 & C. 04700 & 0.04711 & 0.23 \\
\hline 270.00 & 5.15 .85 & 505.76 & -0.02 & C. 05400 & 0.05399 & -0.02 \\
\hline 274.96 & $5=9.06$ & 529.06 & 0.0 & C. .07244 & 0.07244 & 0.0 \\
\hline
\end{tabular}




\section{THERMODYNAMIC PROPERTIES TABLES}

Tables 9 through 12 present values of the thermodynamic variables for the saturated liquid and vapor and superheated vapor states. The saturated liquid at $-40^{\circ} \mathrm{C}$ serves as the reference state for enthalpy and entropy. The following units are used consistently throughout:

$\begin{array}{ll}\text { Pressure, } \mathrm{P} & \mathrm{kPa} \\ \text { Temperature, T } & { }^{\circ} \mathrm{C} \\ \text { Specific volume, } \mathrm{V} & \mathrm{cm}^{3} / \mathrm{g} \\ \text { Specific enthalpy, } \mathrm{h} & \mathrm{kJ} / \mathrm{kg} \\ \text { Specific entropy, s } & \mathrm{kJ} / \mathrm{kg} \cdot \mathrm{K}\end{array}$

The following conversion factors may be used to convert to English units :

\begin{tabular}{lll} 
To convert from & \multicolumn{1}{c}{ To } & Multiply by \\
\hline $\mathrm{kPa}$ & psia & 0.1450 \\
$\mathrm{~cm} / \mathrm{g}$ & $\mathrm{ft}^{3} / 1 \mathrm{~b}_{\mathrm{m}}$ & 0.01602 \\
$\mathrm{~kJ} / \mathrm{kg}$ & $\mathrm{Btu} / 1 \mathrm{~b}_{\mathrm{m}}$ & 0.4302 \\
$\mathrm{~kJ} / \mathrm{kg} \cdot \mathrm{K}$ & $\mathrm{Btu} / 1 \mathrm{~b}_{\mathrm{m}}{ }^{\circ} \mathrm{F}$ & 0.2390
\end{tabular}

Temperature conversion: $\mathrm{T}\left({ }^{\circ} \mathrm{F}\right)=1.8 \times \mathrm{T}\left({ }^{\circ} \mathrm{C}\right)+32$ 
Table 9. The saturation properties of ammonia

\begin{tabular}{|c|c|c|c|c|c|c|c|c|c|c|}
\hline $\mathrm{T}$ & $P$ & $\mathbf{v}_{\mathbf{f}}$ & ${ }^{v_{f g}}$ & $\mathrm{v}_{\mathrm{g}}$ & $h_{f}$ & $h_{f g}$ & $h_{g}$ & $\mathbf{s}_{\mathbf{f}}$ & $s_{f g}$ & $s_{g}$ \\
\hline-20 & 190.6 & 1.504 & 621.7 & 623.3 & 89.78 & 1331 & 1420 & 0.3686 & 5.256 & 5.624 \\
\hline-18 & 208.2 & 1.510 & 572.5 & 574.0 & 98.72 & 1324 & 1423 & 0.4038 & 5.191 & 5.624 \\
\hline-16 & 226.9 & 1.516 & 527.9 & 529.4 & 107.6 & 1318 & 1426 & 0.4382 & 5.126 & 5.565 \\
\hline-14 & 247.0 & 1.522 & 487.3 & 488.8 & 116.8 & 1312 & 1429 & 0.4739 & 5.062 & 5.535 \\
\hline-12 & 268.5 & 1.528 & 450.4 & 452.0 & 125.9 & 1305 & 1431 & 0.5088 & 4.998 & 5.507 \\
\hline-10 & 291.4 & 1.534 & 416.8 & 418.3 & 135.4 & 1298 & 1434 & 0.5449 & 4.933 & 5.478 \\
\hline-8 & 315.8 & 1.540 & 386.3 & 387.8 & 144.4 & 1292 & 1436 & 0.5786 & 4.872 & 5.451 \\
\hline-6 & 341.9 & 1.546 & 358.4 & 360.0 & 153.2 & 1285 & 1439 & 0.6117 & 4.812 & 5.424 \\
\hline-4 & 369.6 & 1.553 & 332.8 & 334.3 & 162.9 & 1278 & 1441 & 0.6476 & 4.749 & 5.396 \\
\hline-2 & 399.0 & 1.559 & 309.3 & 310.9 & 172.4 & 1271 & 1443 & 0.6828 & 4.687 & 5.370 \\
\hline 0 & 430.2 & 1.566 & 288.0 & 289.5 & 181.4 & 1264 & 1446 & 0.7157 & 4.628 & 5.344 \\
\hline 2 & 463.3 & 1.573 & 268.4 & 270.0 & 190.3 & 1257 & 1448 & 0.7480 & 4.570 & 5.318 \\
\hline 4 & 498.3 & 1.580 & 250.2 & 251.8 & 200.0 & 1250 & 1450 & 0.7829 & 4.510 & 5.292 \\
\hline 6 & 535.4 & 1.587 & 233.5 & 235.1 & 209.6 & 1242 & 1452 & 0.8172 & 4.450 & 5.267 \\
\hline 8 & 574.6 & 1.594 & 218.2 & 219.8 & 219.1 & 1235 & 1454 & 0.8509 & 4.391 & 5.242 \\
\hline 10 & 615.9 & 1.601 & 204.0 & 205.6 & 228.5 & 1227 & 1456 & 0.8840 & 4.334 & 5.218 \\
\hline 12 & 659.6 & 1.608 & 191.0 & 192.6 & 237.8 & 1220 & 1458 & 0.9164 & 4.277 & 5.194 \\
\hline 14 & 705.5 & 1.616 & 179.0 & 180.6 & 247.0 & 1212 & 1459 & 0.9483 & 4.222 & 5.170 \\
\hline 16 & 753.9 & 1.623 & 167.7 & 169.3 & 257.0 & 1204 & 1461 & 0.9826 & 4.164 & 5.146 \\
\hline 18 & 804.9 & 1.631 & 157.4 & 159.1 & 265.9 & 1197 & 1462 & 1.013 & 4.110 & 5.123 \\
\hline 20 & 858.4 & 1.639 & 147.8 & 149.4 & 275.7 & 1188 & 1464 & 1.046 & 4.053 & 5.100 \\
\hline 22 & 914.6 & 1.647 & 138.9 & 140.5 & 285.4 & 1180 & 1465 & 1.079 & $\dot{3} .998$ & 5.077 \\
\hline 24 & 973.6 & 1.655 & 130.6 & 132.2 & 294.9 & 1172 & 1467 & 1.111 & 3.943 & 5.054 \\
\hline 26 & 1035 & 1.663 & 122.8 & 124.5 & 304.8 & 1163 & 1468 & 1.144 & 3.888 & 5.032 \\
\hline 28 & 1100 & 1.672 & 115.6 & 117.3 & 314.6 & 1154 & 1469 & 1.176 & 3.833 & 5.009 \\
\hline 30 & 1168 & 1.680 & 108.9 & 110.6 & 324.2 & 1146 & 1470 & 1.207 & 3.780 & 4.987 \\
\hline
\end{tabular}


Table 9 (Continued)

\begin{tabular}{|c|c|c|c|c|c|c|c|c|c|c|}
\hline $\mathrm{T}$ & $P$ & $\mathbf{v}_{\mathbf{f}}$ & $v_{f g}$ & $\mathbf{v}_{\mathbf{g}}$ & $\mathrm{h}_{\mathrm{f}}$ & $h_{f g}$ & $h_{g}$ & $\mathbf{s}_{\mathrm{f}}$ & $s_{f g}$ & $s_{g}$ \\
\hline $\begin{array}{l}32 \\
34 \\
36 \\
38 \\
40\end{array}$ & $\begin{array}{l}1239 \\
1313 \\
1391 \\
1472 \\
1557\end{array}$ & $\begin{array}{l}1.689 \\
1.698 \\
1.707 \\
1.716 \\
1.726\end{array}$ & $\begin{array}{c}102.7 \\
96.82 \\
91.36 \\
86.23 \\
81.43\end{array}$ & $\begin{array}{c}104.4 \\
98.52 \\
93.07 \\
87.94 \\
83.16\end{array}$ & $\begin{array}{l}333.7 \\
343.6 \\
353.4 \\
363.5 \\
373.4\end{array}$ & $\begin{array}{l}1137 \\
1128 \\
1119 \\
1110 \\
1100\end{array}$ & $\begin{array}{l}1471 \\
1472 \\
1473 \\
1473 \\
1474\end{array}$ & $\begin{array}{l}1.238 \\
1.270 \\
1.301 \\
1.333 \\
1.365\end{array}$ & $\begin{array}{l}3.727 \\
3.673 \\
3.620 \\
3.566 \\
3.513\end{array}$ & $\begin{array}{l}4.965 \\
4.943 \\
4.921 \\
4.900 \\
4.878\end{array}$ \\
\hline $\begin{array}{l}42 \\
44 \\
46 \\
48 \\
50\end{array}$ & $\begin{array}{l}1645 \\
1737 \\
1832 \\
1932 \\
2036\end{array}$ & $\begin{array}{l}1.736 \\
1.745 \\
1.756 \\
1.766 \\
1.777\end{array}$ & $\begin{array}{l}76.95 \\
72.75 \\
68.79 \\
65.06 \\
61.55\end{array}$ & $\begin{array}{l}78.68 \\
74.50 \\
70.55 \\
66.82 \\
63.33\end{array}$ & $\begin{array}{l}383.3 \\
393.0 \\
403.1 \\
413.5 \\
423.7\end{array}$ & $\begin{array}{l}1091 \\
1081 \\
1071 \\
1061 \\
1051\end{array}$ & $\begin{array}{l}1474 \\
1474 \\
1474 \\
1474 \\
1474\end{array}$ & $\begin{array}{l}1.396 \\
1.426 \\
1.457 \\
1.489 \\
1.520\end{array}$ & $\begin{array}{l}3.461 \\
3.409 \\
3.357 \\
3.304 \\
3.251\end{array}$ & $\begin{array}{l}4.857 \\
4.835 \\
4.814 \\
4.793 \\
4.771\end{array}$ \\
\hline $\begin{array}{l}52 \\
54 \\
56 \\
58 \\
60\end{array}$ & $\begin{array}{l}2143 \\
2255 \\
2372 \\
2492 \\
2618\end{array}$ & $\begin{array}{l}1.788 \\
1.799 \\
1.810 \\
1.822 \\
1.834\end{array}$ & $\begin{array}{l}58.29 \\
55.17 \\
52.25 \\
49.50 \\
46.88\end{array}$ & $\begin{array}{l}60.08 \\
56.97 \\
54.106 \\
51.32 \\
48.72\end{array}$ & $\begin{array}{l}433.4 \\
443.9 \\
454.2 \\
464.5 \\
475.0\end{array}$ & $\begin{array}{r}1041 \\
1030 \\
1019 \\
1008 \\
996.7\end{array}$ & $\begin{array}{l}1474 \\
1474 \\
1473 \\
1473 \\
1472\end{array}$ & $\begin{array}{l}1.550 \\
1.581 \\
1.612 \\
1.642 \\
1.673\end{array}$ & $\begin{array}{l}3.201 \\
3.148 \\
3.096 \\
3.044 \\
2.992\end{array}$ & $\begin{array}{l}4.750 \\
4.729 \\
4.708 \\
4.686 \\
4.665\end{array}$ \\
\hline $\begin{array}{l}62 \\
64 \\
66 \\
68 \\
70\end{array}$ & $\begin{array}{l}2748 \\
2883 \\
3022 \\
3167 \\
3317\end{array}$ & $\begin{array}{l}1.847 \\
1.860 \\
1.873 \\
1.886 \\
1.900\end{array}$ & $\begin{array}{l}44.43 \\
42.09 \\
39.89 \\
37.81 \\
35.85\end{array}$ & $\begin{array}{l}46.27 \\
43.75 \\
41.76 \\
39.70 \\
37.75\end{array}$ & $\begin{array}{l}485.4 \\
496.1 \\
506.7 \\
517.2 \\
527.6\end{array}$ & $\begin{array}{l}985.3 \\
973.4 \\
961: 5 \\
949.5 \\
937.5\end{array}$ & $\begin{array}{l}1471 \\
1470 \\
1468 \\
1467 \\
1465\end{array}$ & $\begin{array}{l}1.704 \\
1.735 \\
1.765 \\
1.795 \\
1.825\end{array}$ & $\begin{array}{l}2.940 \\
2.887 \\
2.835 \\
2.783 \\
2.732\end{array}$ & $\begin{array}{l}4.644 \\
4.622 \\
4.600 \\
4.579 \\
4.557\end{array}$ \\
\hline $\begin{array}{l}72 \\
74 \\
76 \\
78 \\
80\end{array}$ & $\begin{array}{l}3472 \\
3632 \\
3798 \\
3970 \\
4147\end{array}$ & $\begin{array}{l}1.915 \\
1.930 \\
1.945 \\
1.961 \\
1.978\end{array}$ & $\begin{array}{l}33.97 \\
32.19 \\
30.50 \\
28.91 \\
27.37\end{array}$ & $\begin{array}{l}35.88 \\
34.12 \\
32.45 \\
30.87 \\
29.35\end{array}$ & $\begin{array}{l}538.7 \\
549.6 \\
560.5 \\
571.2 \\
582.6\end{array}$ & $\begin{array}{l}924.5 \\
911.5 \\
898.4 \\
885.2 \\
871.0\end{array}$ & $\begin{array}{l}1463 \\
1461 \\
1459 \\
1456 \\
1454\end{array}$ & $\begin{array}{l}1.856 \\
1.887 \\
1.917 \\
1.947 \\
1.978\end{array}$ & $\begin{array}{l}2.679 \\
2.626 \\
2.573 \\
2.521 \\
2.466\end{array}$ & $\begin{array}{l}4.535 \\
4.513 \\
4.490 \\
4.468 \\
4.445\end{array}$ \\
\hline $\begin{array}{l}82 \\
84 \\
86 \\
88 \\
90\end{array}$ & $\begin{array}{l}4330 \\
4519 \\
4715 \\
4916 \\
5124\end{array}$ & $\begin{array}{l}1.995 \\
2.013 \\
2.032 \\
2.051 \\
2.072\end{array}$ & $\begin{array}{l}25.92 \\
24.54 \\
23.21 \\
21.94 \\
20.74\end{array}$ & $\begin{array}{l}27.91 \\
26.55 \\
25.24 \\
22.99 \\
22.82\end{array}$ & $\begin{array}{l}594.0 \\
605.2 \\
617.0 \\
628.8 \\
640.5\end{array}$ & $\begin{array}{l}856.7 \\
842.3 \\
826.9 \\
811.3 \\
795.6\end{array}$ & $\begin{array}{l}1451 \\
1447 \\
1444 \\
1440 \\
1436\end{array}$ & $\begin{array}{l}2.009 \\
2.040 \\
2.072 \\
2.103 \\
2.134\end{array}$ & $\begin{array}{l}2.412 \\
2.358 \\
2.302 \\
2.246 \\
2.191\end{array}$ & $\begin{array}{l}4.422 \\
4.398 \\
4.374 \\
4.350 \\
4.325\end{array}$ \\
\hline
\end{tabular}


Table 9 (Continued)

\begin{tabular}{|c|c|c|c|c|c|c|c|c|c|c|}
\hline $\mathrm{T}$ & $\mathbf{P}$ & $v_{f}$ & $v_{f g}$ & $v_{\cdot g}$ & $h_{f}$ & $h_{f g}$ & $h_{g}$ & $s_{f}$ & ${ }^{s_{f g}}$ & $\mathrm{~s}_{\mathrm{g}}$ \\
\hline 92 & 5338 & 2.09 .3 & 19.61 & $\approx 1.70$ & 652.0 & 779.8 & 1432 & 2.165 & 2.136 & 4.301 \\
\hline 94 & 5559 & 2.115 & 18.50 & 20.62 & 664.3 & 762.8 & -427 & 2.197 & 2.078 & 4.275 \\
\hline 96 & 5787 & 2.139 & 17.45 & 19.59 & 676.4 & 745.7 & ¡4222 & 2.229 & 2.020 & 4.249 \\
\hline 98 & 6022 & 2.163 & 16.43 & 18.59 & 689.2 & 727.4 & $\Xi 417$ & 2.262 & 1.960 & 4.222 \\
\hline 100 & 6264 & 2.190 & 15.46 & 17.65 & 701.8 & 708.9 & -411 & 2.295 & 1.900 & 4.194 \\
\hline 102 & 6513 & 2.217 & 14.52 & 16.73 & 715.1 & $689 . .2$ & 1404 & 2.329 & 1.837 & 4.166 \\
\hline 104 & 6769 & 2.247 & 13.62 & 15.86 & 728.3 & 669.2 & 1398 & 2. 362 & 1.774 & 4.137 \\
\hline 106 & 7033 & 2.279 & 12.74 & 15.02 & 742.1 & 648.0 & 1390 & 2.397 & 1.709 & 4.106 \\
\hline 108 & 7304 & 2.312 & 11.90 & 14.22 & 755.9 & 626.4 & 1382 & 2.432 & 1.643 & 4.075 \\
\hline 110 & 7584 & 2.349 & 11.09 & 13.43 & 770.3 & 603.4 & 1374 & 2.468 & 1.575 & 4.043 \\
\hline 112 & 7871 & 2.389 & 10.29 & 12.68 & 785.2 & 579.1 & 1364 & 2.505 & 1.504 & 4.008 \\
\hline 114 & 8167 & 2.433 & 9.505 & 11.94 & 800.7 & 553.2 & 1354 & 2.543 & 1.429 & 3.972 \\
\hline 116 & 8471 & 2.481 & 8.756 & 11.24 & 816.3 & 526.8 & 1343 & 2.582 & 1.354 & 3.935 \\
\hline 118 & 8783 & 2.535 & 8.005 & 10.54 & 833.1 & 497.6 & 1331 & 2.623 & 1.272 & 3.395 \\
\hline 120 & 9104 & 2.596 & 7.249 & 9.845 & 851.0 & 465.6 & 1317 & 2.666 & 1.184 & 3.350 \\
\hline 122 & 9434 & 2.666 & 6.489 & 7.156 & 870.0 & 430.6 & 1301 & 2.712 & 1.090 & 3.302 \\
\hline 124 & 9772 & 2.750 & 5.692 & 3.443 & 891.1 & 390.1 & 1281 & 2.763 & 0.9821 & 3.745 \\
\hline 126 & 10120 & 2.854 & 4.884 & 7.737 & 913.6 & 345.5 & 1259 & 2.817 & 0.8657 & 3.583 \\
\hline 128 & 10480 & 2.989 & 3.968 & $\overline{5.958}$ & 939.9 & 289.9 & 1230 & 2.880 & 0.7226 & 3.503 \\
\hline 130 & 10850 & 3.192 & 2.812 & р.003 & 972.7 & 212.0 & 1185 & 2.959 & 0.5258 & 3.485 \\
\hline 132 & 11220 & 3.680 & 1.003 & 4.683 & 1024 & 78.02 & 1102 & 3.083 & 0.1926 & 3.276 \\
\hline 132.3 & 11280 & C.247 & 0.0 & 4.247 & 1068 & 0.0 & 1068 & 3.190 & 0.0 & $3 .: 90$ \\
\hline
\end{tabular}


Table 10. Thermodynamic properties of ammonia in the superheated vapor state Temperature range: -50 to $0^{\circ} \mathrm{C}$

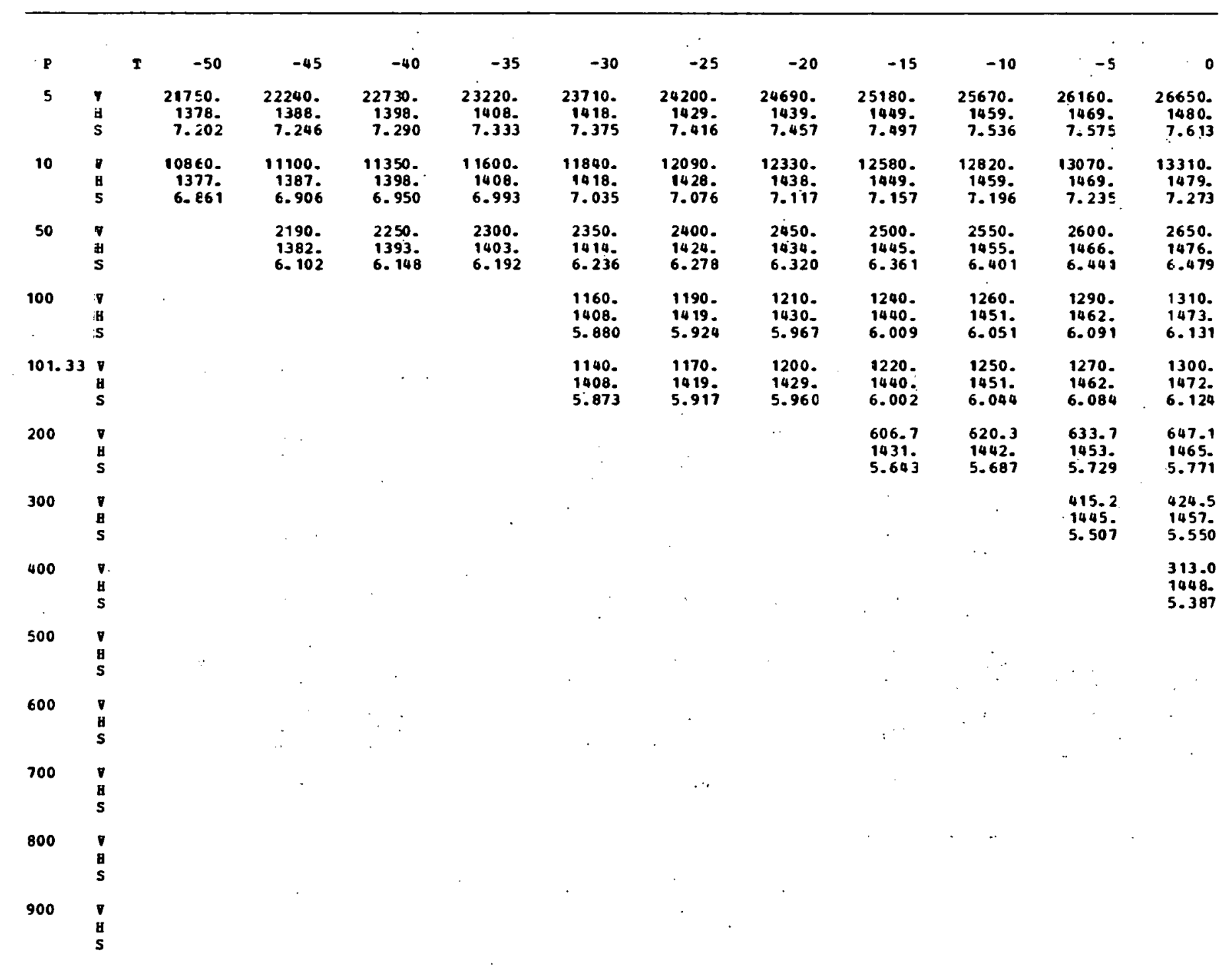


Table 10 (Continued)

Temperature range: 5 to $55^{\circ} \mathrm{C}$

\begin{tabular}{|c|c|c|c|c|c|c|c|c|c|c|c|c|}
\hline P & & $I$ & 10 & 15 & 20 & 25 & 30 & 35 & 40 & 45 & 50 & 95 \\
\hline 5 & $\begin{array}{l}8 \\
4 \\
5\end{array}$ & $\begin{array}{l}27140 . \\
7490^{\circ} \\
7.650^{\circ}\end{array}$ & $\begin{array}{r}27630 \\
1500 \\
7.687\end{array}$ & $\begin{array}{r}28120 \\
1511 \\
7.723\end{array}$ & $\begin{array}{r}28600 \\
1521 \\
7.759\end{array}$ & $\begin{array}{c}29090 . \\
1532 . \\
7.795\end{array}$ & $\begin{array}{r}29580 \\
1542= \\
7.829\end{array}$ & $\begin{array}{r}32070 \\
1553 \\
7.864\end{array}$ & $\begin{array}{c}30560 \\
1563 \\
7.898\end{array}$ & $\begin{array}{c}31050 . \\
1574 . \\
7.932\end{array}$ & $\begin{array}{l}31540 . \\
1585 \\
7.965\end{array}$ & $\begin{array}{c}32030 \\
1595 \\
7.998\end{array}$ \\
\hline 10. & $\begin{array}{l}7 \\
4 \\
\text { S }\end{array}$ & $\begin{array}{l}13560 . \\
1490 \\
7.311\end{array}$ & $\begin{array}{l}13800 \\
1500 \\
7.348\end{array}$ & $\begin{array}{l}14050 . \\
1510 . \\
7.384\end{array}$ & $\begin{array}{r}14290 . \\
1521 . \\
7.420\end{array}$ & $\begin{array}{l}10540^{\circ} \\
1531 . \\
7.456\end{array}$ & $\begin{array}{r}14780 \\
1542 . \\
7.491\end{array}$ & $\begin{array}{r}15030 \\
1552 \\
7.525\end{array}$ & $\begin{array}{l}15270 \\
1563 . \\
7.559\end{array}$ & $\begin{array}{r}15520 . \\
1574 . \\
7.593\end{array}$ & $\begin{array}{l}15760 \\
1584 \\
7.62 \epsilon\end{array}$ & $\begin{array}{c}16010 \\
1595 \\
7.659\end{array}$ \\
\hline$\leq 0$ & $\begin{array}{l}\text { D } \\
\text { s }\end{array}$ & $\begin{array}{l}27 \mathrm{co} . \\
1487 . \\
6.518\end{array}$ & $\begin{array}{l}2750 . \\
1497: \\
6.555\end{array}$ & $\begin{array}{l}2800 . \\
1508 . \\
6.592\end{array}$ & $\begin{array}{l}2850 . \\
1519 . \\
6.628\end{array}$ & $\begin{array}{l}2900 . \\
1529 . \\
6.664\end{array}$ & $\begin{array}{l}2950 . \\
1540 . \\
6.700\end{array}$ & $\begin{array}{l}2990 . \\
1550 . \\
6.735\end{array}$ & $\begin{array}{l}3040 \\
1561 \\
6.769\end{array}$ & $\begin{array}{l}3090 \\
1572 \\
6.803\end{array}$ & $\begin{array}{l}3140 . \\
1583 . \\
6.836\end{array}$ & $\begin{array}{l}3190 . \\
1593 . \\
6.870\end{array}$ \\
\hline 100 & $\begin{array}{l}\text { D } \\
\text { i } \\
\text { s }\end{array}$ & $\begin{array}{l}1340 . \\
1483 \\
6.170\end{array}$ & $\begin{array}{l}1360 . \\
1494 . \\
6.208\end{array}$ & $\begin{array}{l}1390 . \\
1505 . \\
6.245\end{array}$ & $\begin{array}{l}1420 . \\
1516 . \\
6.282\end{array}$ & $\begin{array}{l}1440 . \\
1526 . \\
6.319\end{array}$ & $\begin{array}{l}1470 . \\
1537 . \\
6.355\end{array}$ & $\begin{array}{l}1490 . \\
1548 . \\
5.390\end{array}$ & $\begin{array}{l}1520 \\
1559 . \\
6.425\end{array}$ & $\begin{array}{l}1540 . \\
1570 \\
6.459\end{array}$ & $\begin{array}{l}1570 \\
1580 \\
6.493\end{array}$ & $\begin{array}{l}1590 . \\
1591 . \\
6.526\end{array}$ \\
\hline 101.33 & $\begin{array}{l}P \\
\mathbf{S} \\
\mathbf{S}\end{array}$ & $\begin{array}{l}1320 \\
1483 \\
6.163\end{array}$ & $\begin{array}{l}1350 . \\
1494 \\
6.209\end{array}$ & $\begin{array}{l}1370 . \\
1505 \\
6.239\end{array}$ & $\begin{array}{l}1400 . \\
1515 \\
6.276\end{array}$ & $\begin{array}{l}1420 . \\
1526 . \\
6.312\end{array}$ & $\begin{array}{l}1450 \\
1537 \\
6.348\end{array}$ & $\begin{array}{l}1470 \\
1548 . \\
5.383\end{array}$ & $\begin{array}{l}1500 \\
1559 . \\
6.418\end{array}$ & $\begin{array}{l}1520 \\
1569 \\
6.453\end{array}$ & $\begin{array}{l}1540 \\
1580 \\
6.486\end{array}$ & $\begin{array}{l}1570 . \\
1591 . \\
6.520\end{array}$ \\
\hline 200 & $\begin{array}{l}\mathbf{y} \\
\mathbf{y} \\
\mathrm{s}\end{array}$ & $\begin{array}{l}660.3 \\
1476 . \\
5.811\end{array}$ & $\begin{array}{l}673.5 \\
1087 . \\
5.85 i\end{array}$ & $\begin{array}{l}686.6 \\
1498 . \\
5.890\end{array}$ & $\begin{array}{l}699.6 \\
1509 . \\
5.928\end{array}$ & $\begin{array}{l}712.6 \\
1520 . \\
5.966\end{array}$ & $\begin{array}{l}725.5 \\
1532 . \\
6.003\end{array}$ & $\begin{array}{l}738.4 \\
1543 . \\
5.039\end{array}$ & $\begin{array}{l}751.2 \\
1554 . \\
6.075\end{array}$ & $\begin{array}{l}764.0 \\
1565 . \\
6.110\end{array}$ & $\begin{array}{l}776.7 \\
1576 . \\
6.144\end{array}$ & $\begin{array}{l}789.4 \\
1587 . \\
6.179\end{array}$ \\
\hline 300 & $\begin{array}{l}\mathbf{D} \\
\mathbf{H} \\
\mathbf{S}\end{array}$ & $\begin{array}{l}433.7 \\
1468 . \\
5.593\end{array}$ & $\begin{array}{l}442.9 \\
1480 . \\
5.634\end{array}$ & $\begin{array}{l}452.0 \\
1492 . \\
5.675\end{array}$ & $\begin{array}{l}460.9 \\
1503 . \\
5.714\end{array}$ & $\begin{array}{l}969.9 \\
1514 . \\
5.753\end{array}$ & $\begin{array}{l}478.7 \\
1526 . \\
5.791\end{array}$ & $\begin{array}{l}487.6 \\
1537 . \\
5.828\end{array}$ & $\begin{array}{l}496.3 \\
1549 . \\
5.865\end{array}$ & $\begin{array}{l}505.0 \\
1560 . \\
5.901\end{array}$ & $\begin{array}{l}513.7 \\
1571 . \\
5.93 E\end{array}$ & $\begin{array}{l}522.4 \\
1583 . \\
5.971\end{array}$ \\
\hline 400 & $\begin{array}{l}y \\
y \\
s\end{array}$ & $\begin{array}{l}320.3 \\
1460 \\
5.431\end{array}$ & $\begin{array}{l}327.4 \\
1473 . \\
5.474\end{array}$ & $\begin{array}{l}334.5 \\
1485 . \\
5.516\end{array}$ & $\begin{array}{l}341.5 \\
1496 . \\
5.557\end{array}$ & $\begin{array}{l}348.4 \\
1508 . \\
5.597\end{array}$ & $\begin{array}{l}355.3 \\
1520 . \\
5.636\end{array}$ & $\begin{array}{l}362.1 \\
1532 . \\
5.675\end{array}$ & $\begin{array}{l}368.8 \\
1543 . \\
5.712\end{array}$ & $\begin{array}{l}375.5 \\
1555 . \\
5.749\end{array}$ & $\begin{array}{l}382.2 \\
1567 . \\
5.785\end{array}$ & $\begin{array}{l}388.8 \\
1578 . \\
5.820\end{array}$ \\
\hline 500 & $\begin{array}{l}\mathbf{D} \\
\mathbf{H} \\
\mathrm{S}\end{array}$ & $\begin{array}{l}252.1 \\
1452 . \\
5.300\end{array}$ & $\begin{array}{l}258.1 \\
1465 . \\
5.345\end{array}$ & $\begin{array}{l}263.9 \\
1477 . \\
5.389\end{array}$ & $\begin{array}{l}269.7 \\
1490 . \\
5.431\end{array}$ & $\begin{array}{l}275.5 \\
1502 . \\
5.472\end{array}$ & $\begin{array}{l}281.1 \\
1514 . \\
5.513\end{array}$ & $\begin{array}{l}286.7 \\
1526 . \\
5.552\end{array}$ & $\begin{array}{l}292.3 \\
1538 . \\
5.591\end{array}$ & $\begin{array}{l}297.8 \\
1550 . \\
5.628\end{array}$ & $\begin{array}{l}303.2 \\
1562 . \\
5.665\end{array}$ & $\begin{array}{l}308.6 \\
1574 . \\
5.701\end{array}$ \\
\hline 600 & $\begin{array}{l}v \\
H \\
S\end{array}$ & & $\begin{array}{l}211.7 \\
1457 . \\
5.234\end{array}$ & $\begin{array}{l}216.8 \\
1470 . \\
5.280\end{array}$ & $\begin{array}{l}221.8 \\
1483 . \\
5.324\end{array}$ & $\begin{array}{l}226.8 \\
1496 . \\
5.367\end{array}$ & $\begin{array}{l}231.6 \\
1508 . \\
5.409\end{array}$ & $\begin{array}{l}236.4 \\
1520 . \\
5.449\end{array}$ & $\begin{array}{l}241.2 \\
1533 . \\
5.489\end{array}$ & $\begin{array}{l}245.9 \\
1545 . \\
5.527\end{array}$ & $\begin{array}{l}250.5 \\
1557 . \\
5.565\end{array}$ & $\begin{array}{l}255.2 \\
1569 . \\
5.602\end{array}$ \\
\hline 700 & $\begin{array}{l}7 \\
8 \\
5\end{array}$ & & & $\begin{array}{l}183.0 \\
1462 . \\
5.184\end{array}$ & $\begin{array}{l}187.5 \\
1476 . \\
5.230\end{array}$ & $\begin{array}{l}191.9 \\
1489 . \\
5.275\end{array}$ & $\begin{array}{l}196.2 \\
1502 . \\
5.318\end{array}$ & $\begin{array}{l}200.5 \\
1515 . \\
5.360\end{array}$ & $\begin{array}{l}204.7 \\
1527 . \\
5.400\end{array}$ & $\begin{array}{l}208.8 \\
1540^{\circ} \\
5.440\end{array}$ & $\begin{array}{l}212.9 \\
1552 . \\
5.478\end{array}$ & $\begin{array}{l}216.9 \\
1564 . \\
5.516\end{array}$ \\
\hline 800 & $\begin{array}{l}v \\
\theta \\
\mathbf{s}\end{array}$ & & & & $\begin{array}{l}161.7 \\
1468 . \\
5.146\end{array}$ & $\begin{array}{l}165.7 \\
1482 . \\
5.192\end{array}$ & $\begin{array}{l}169.6 \\
1495 . \\
5.236\end{array}$ & $\begin{array}{l}173.5 \\
1509 . \\
5.280\end{array}$ & $\begin{array}{l}177.2 \\
1522 . \\
5.321\end{array}$ & $\begin{array}{l}181.0 \\
1534 . \\
5.362\end{array}$ & $\begin{array}{l}184.6 \\
1547 . \\
5.401\end{array}$ & $\begin{array}{l}188.2 \\
1560 . \\
5.440\end{array}$ \\
\hline 903 & $\begin{array}{l}v \\
u \\
s\end{array}$ & & & & & $\begin{array}{l}145.3 \\
1475 . \\
5.116\end{array}$ & $\begin{array}{l}148.9 \\
1489 . \\
5.162\end{array}$ & $\begin{array}{l}152.4 \\
1502 \\
5.207\end{array}$ & $\begin{array}{l}155.9 \\
1516 . \\
5.250\end{array}$ & $\begin{array}{l}159.3 \\
1529 \\
5.291\end{array}$ & $\begin{array}{l}162.6 \\
1542 . \\
5.332\end{array}$ & $\begin{array}{l}165.9 \\
1555 . \\
5.371\end{array}$ \\
\hline
\end{tabular}


Table 10 (Continued)

Temperature range: 5 to $55^{\circ} \mathrm{C}$

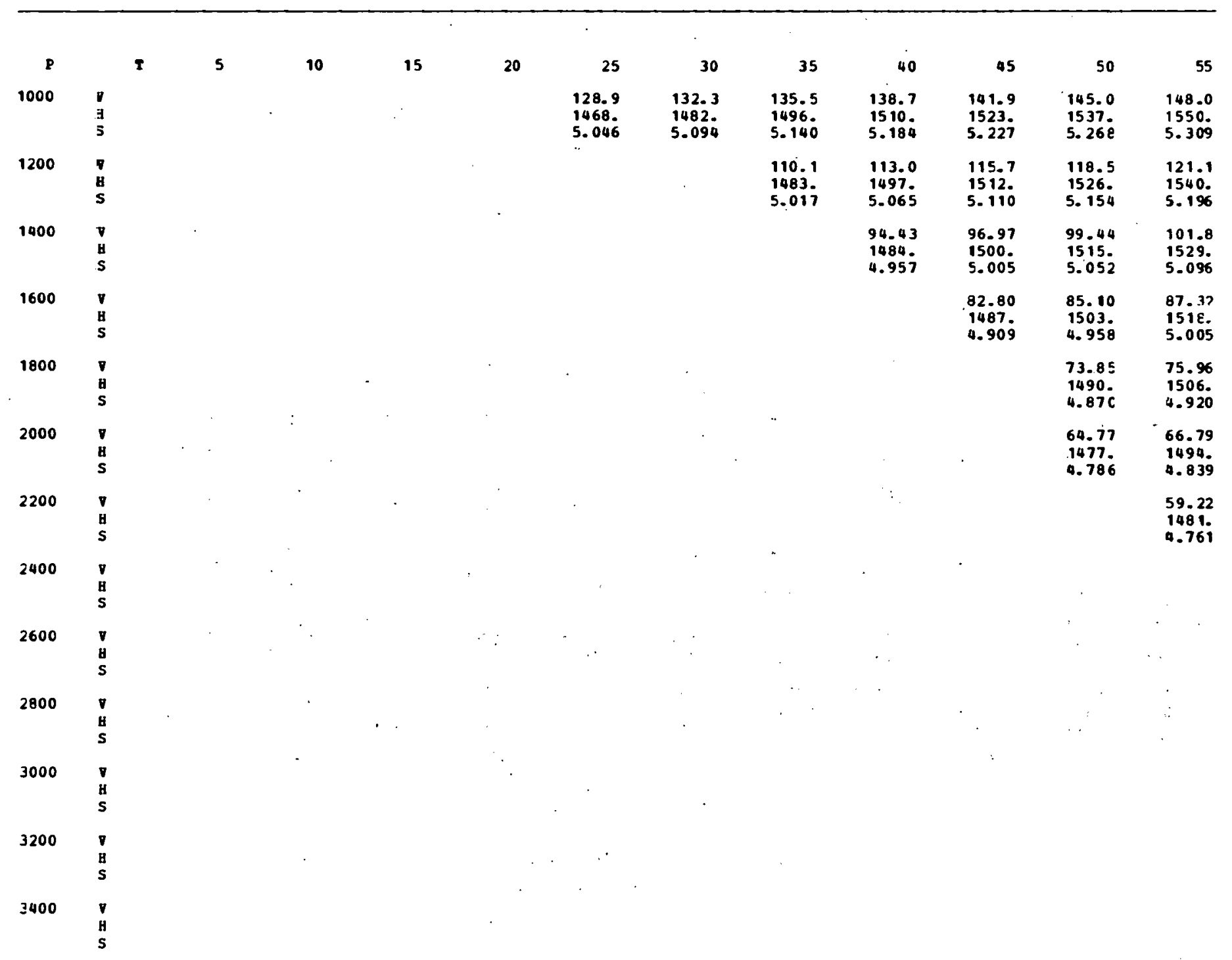


Table 10 (Continued)

Temperature range: 60 to $110^{\circ} \mathrm{C}$

\begin{tabular}{|c|c|c|c|c|c|c|c|c|c|c|c|c|c|}
\hline p & & $\mathbf{T}$ & 60 & 65 & 70 & 75 & 80 & 85 & 90 & 95 & 100 & 105 & 110 \\
\hline 5 & $\begin{array}{l}\nabla \\
\mathbf{u} \\
\mathbf{s}\end{array}$ & & $\begin{array}{r}32510 . \\
1606 \\
3.030\end{array}$ & $\begin{array}{r}33000 \\
1617 \\
8.062\end{array}$ & $\begin{array}{r}33490 . \\
1628 . \\
8.094\end{array}$ & $\begin{array}{r}33980 \\
1638 \\
8.126\end{array}$ & $\begin{array}{r}34470 \\
1649 \\
8.157\end{array}$ & $\begin{array}{r}34960 \\
1660 \\
8.187\end{array}$ & $\begin{array}{l}35450 \\
1671 \\
E .218\end{array}$ & $\begin{array}{c}3 \leq 930 \\
1682 \\
\varepsilon .248\end{array}$ & $\begin{array}{l}36420 . \\
1694- \\
8.278\end{array}$ & $\begin{array}{r}36910 . \\
1705 . \\
8.308\end{array}$ & $\begin{array}{r}37400 . \\
1716 . \\
8.337\end{array}$ \\
\hline 10 & $\begin{array}{l}\nabla \\
\mathrm{a} \\
\mathrm{S}\end{array}$ & & $\begin{array}{r}16250 . \\
1606 . \\
7.691\end{array}$ & $\begin{array}{c}16500 \\
1617 \\
7.724\end{array}$ & $\begin{array}{r}16740 . \\
.1627 \\
.7 .755\end{array}$ & $\begin{array}{r}16980 \\
1638 \\
7.787\end{array}$ & $\begin{array}{r}17230 \\
1699 \\
7.818\end{array}$ & $\begin{array}{r}17470 \\
1660 \\
7.849\end{array}$ & $\begin{array}{r}17720 \\
1671 \\
7.879\end{array}$ & $\begin{array}{r}17960 \\
1682 \\
7.910\end{array}$ & $\begin{array}{c}18210 . \\
1693 . \\
7.940\end{array}$ & $\begin{array}{c}18450 \\
1705 \\
7.969\end{array}$ & $\begin{array}{r}18700 . \\
1716 . \\
7.999\end{array}$ \\
\hline 50 & $\begin{array}{l}\nabla \\
H \\
S\end{array}$ & & $\begin{array}{l}3240 . \\
1604 . \\
6.902\end{array}$ & $\begin{array}{l}3290 . \\
1615 . \\
6.935\end{array}$ & $\begin{array}{l}3340 . \\
1626 \\
6.967\end{array}$ & $\begin{array}{l}3390 . \\
1637 . \\
6.998\end{array}$ & $\begin{array}{l}3440 . \\
1648 . \\
7.030\end{array}$ & $\begin{array}{l}3490 . \\
1659 \\
7.06 i\end{array}$ & $\begin{array}{l}3540 . \\
1670 . \\
7.091\end{array}$ & $\begin{array}{l}\equiv 590 . \\
1681 . \\
7.122\end{array}$ & $\begin{array}{l}3630 . \\
1692 . \\
7.152\end{array}$ & $\begin{array}{l}3680 . \\
1703 \\
7.181\end{array}$ & $\begin{array}{l}3730 . \\
1715 . \\
7.211\end{array}$ \\
\hline 100 & $\begin{array}{l}\mathbf{D} \\
\mathbf{B} \\
\mathbf{S}\end{array}$ & & $\begin{array}{l}1620 . \\
1602 . \\
6.560\end{array}$ & $\begin{array}{l}1640 . \\
1613 . \\
6.592\end{array}$ & $\begin{array}{l}1660 . \\
1624 . \\
6.624\end{array}$ & $\begin{array}{l}1690 . \\
1635 . \\
6.656\end{array}$ & $\begin{array}{l}1710 . \\
1646 . \\
6.688\end{array}$ & $\begin{array}{l}1740 . \\
1657 \\
6.719\end{array}$ & $\begin{array}{l}1760 . \\
1668 . \\
\epsilon .750\end{array}$ & $\begin{array}{l}1790 \\
1680 \\
6.780\end{array}$ & $\begin{array}{l}1810 . \\
1691 . \\
6.810\end{array}$ & $\begin{array}{l}1840 . \\
1702 . \\
6.840\end{array}$ & $\begin{array}{l}1860 . \\
1713 . \\
6.870\end{array}$ \\
\hline 109.33 & $\begin{array}{l}\text { D } \\
\text { B } \\
\text { S }\end{array}$ & & $\begin{array}{l}1590 . \\
1602 . \\
6.553\end{array}$ & $\begin{array}{l}1620 . \\
1613 . \\
\epsilon .586\end{array}$ & $\begin{array}{l}1640 . \\
1624 . \\
6.618\end{array}$ & $\begin{array}{l}1670 . \\
1635 . \\
6.650\end{array}$ & $\begin{array}{l}1690 . \\
1646 . \\
6.681\end{array}$ & $\begin{array}{l}1720 . \\
1657 . \\
6.712\end{array}$ & $\begin{array}{l}1740 . \\
1668 . \\
6.743\end{array}$ & $\begin{array}{l}1770 . \\
1679 \\
6.774\end{array}$ & $\begin{array}{l}1790 . \\
1691 . \\
6.804\end{array}$ & $\begin{array}{l}1810 . \\
1702 . \\
6.834\end{array}$ & $\begin{array}{l}1840 . \\
1713 . \\
6.863\end{array}$ \\
\hline 200 & $\begin{array}{l}\nabla \\
\text { d } \\
\text { s }\end{array}$ & & $\begin{array}{l}102.1 \\
1598 . \\
6.212\end{array}$ & $\begin{array}{l}814.7 \\
1609 . \\
6.245\end{array}$ & $\begin{array}{l}827.4 \\
1620 . \\
6.278\end{array}$ & $\begin{array}{l}840.0 \\
1632 . \\
6.310\end{array}$ & $\begin{array}{l}852.5 \\
1643 . \\
6.342\end{array}$ & $\begin{array}{l}865.1 \\
1654 . \\
6.374\end{array}$ & $\begin{array}{l}877.6 \\
1665 . \\
6405\end{array}$ & $\begin{array}{l}E 90.1 \\
1676 . \\
E .436\end{array}$ & $\begin{array}{l}902.6 \\
1688 . \\
6.466\end{array}$ & $\begin{array}{l}915.1 \\
1699 . \\
6.497\end{array}$ & $\begin{array}{l}927.6 \\
1710 . \\
6.526\end{array}$ \\
\hline 300 & $\begin{array}{l}: \\
\mathbf{B} \\
\mathbf{s}\end{array}$ & & $\begin{array}{l}531.0 \\
1594 . \\
6.005\end{array}$ & $\begin{array}{l}539.6 \\
1605 . \\
6.039\end{array}$ & $\begin{array}{l}548.1 \\
1617 . \\
6.072\end{array}$ & $\begin{array}{l}556.7 \\
1628 . \\
6.105\end{array}$ & $\begin{array}{l}565.2 \\
1639 . \\
6.137\end{array}$ & $\begin{array}{l}573.7 \\
1651 . \\
6.169\end{array}$ & $\begin{array}{l}582.1 \\
1662 . \\
6.201\end{array}$ & $\begin{array}{l}590.6 \\
1673 . \\
6.232\end{array}$ & $\begin{array}{l}599.0 \\
1685 . \\
6.263\end{array}$ & $\begin{array}{l}607.4 \\
1696 \\
6.293\end{array}$ & $\begin{array}{l}615.8 \\
1708 . \\
6.323\end{array}$ \\
\hline 400 & $\begin{array}{l}\nabla \\
\text { y } \\
5\end{array}$ & & $\begin{array}{l}395.4 \\
1590 . \\
5.855\end{array}$ & $\begin{array}{l}402.0 \\
1601 . \\
5.890\end{array}$ & $\begin{array}{l}408.5 \\
1613 . \\
5.923\end{array}$ & $\begin{array}{l}415.0 \\
1624 . \\
5.957\end{array}$ & $\begin{array}{l}421.5 \\
1636 . \\
5.989\end{array}$ & $\begin{array}{l}427.9 \\
1647 . \\
6.022\end{array}$ & $\begin{array}{l}434.4 \\
1659 . \\
\epsilon 054\end{array}$ & $\begin{array}{l}440.8 \\
1670 . \\
6.085\end{array}$ & $\begin{array}{l}447.2 \\
1682 . \\
6.116\end{array}$ & $\begin{array}{l}453.6 \\
1693 . \\
6.147\end{array}$ & $\begin{array}{l}460.0 \\
1705 . \\
6.077\end{array}$ \\
\hline 500 & $\begin{array}{l}7 \\
8 \\
5\end{array}$ & & $\begin{array}{l}314.0 \\
\text { i585. } \\
5.737\end{array}$ & $\begin{array}{l}319.4 \\
1597 . \\
5.772\end{array}$ & $\begin{array}{l}324.7 \\
1609 . \\
5.806\end{array}$ & $\begin{array}{l}330.0 \\
1620 . \\
5.840\end{array}$ & $\begin{array}{l}335.2 \\
1632 . \\
5.873\end{array}$ & $\begin{array}{l}340.5 \\
1644 . \\
5.906\end{array}$ & $\begin{array}{l}345.7 \\
1655 . \\
5938\end{array}$ & $\begin{array}{l}350.9 \\
1667 . \\
5.970\end{array}$ & $\begin{array}{l}356.1 \\
1679 . \\
6.00 i\end{array}$ & $\begin{array}{l}361.3 \\
1690 . \\
6.033\end{array}$ & $\begin{array}{l}366.4 \\
1702 . \\
6.063\end{array}$ \\
\hline 600 & $\begin{array}{l}y \\
\mathbf{B} \\
\mathrm{s}\end{array}$ & & $\begin{array}{l}259.7 \\
15 E 1 . \\
3.638\end{array}$ & $\begin{array}{l}264.3 \\
1593 . \\
5.674 .\end{array}$ & $\begin{array}{l}268.8 \\
1605 . \\
5.709\end{array}$ & $\begin{array}{l}273.3 \\
1617 . \\
5.743\end{array}$ & $\begin{array}{l}277.7 \\
1628 \\
5.777\end{array}$ & $\begin{array}{l}282.2 \\
1640^{\circ} \\
5.810\end{array}$ & $\begin{array}{l}286.6 \\
1652 . \\
5.842\end{array}$ & $\begin{array}{l}291.0 \\
8664 . \\
5.875\end{array}$ & $\begin{array}{l}295.4 \\
1676 . \\
5.906\end{array}$ & $\begin{array}{l}299.7 \\
1687 . \\
5.938\end{array}$ & $\begin{array}{l}304.1 \\
1699 . \\
5.969\end{array}$ \\
\hline 700 & $\begin{array}{l}D \\
y \\
5\end{array}$ & & $\begin{array}{l}220.9 \\
1577 \\
5.553\end{array}$ & $\begin{array}{l}224.9 \\
1589 . \\
5.589\end{array}$ & $\begin{array}{l}228 . B \\
1601 . \\
5.625\end{array}$ & $\begin{array}{l}232.8 \\
1613 . \\
5.659\end{array}$ & $\begin{array}{l}236.6 \\
1625 . \\
5.694\end{array}$ & $\begin{array}{l}240.5 \\
1637 \\
5.727\end{array}$ & $\begin{array}{l}244.3 \\
1649 . \\
5.760\end{array}$ & $\begin{array}{l}248.2 \\
1661 . \\
5.793\end{array}$ & $\begin{array}{l}252.0 \\
1673 . \\
5.825\end{array}$ & $\begin{array}{l}255.7 \\
1684 . \\
5.857\end{array}$ & $\begin{array}{l}259.5 \\
1696 . \\
5.888\end{array}$ \\
\hline 800 & $\begin{array}{l}y \\
y \\
\text { S }\end{array}$ & & $\begin{array}{l}191.8 \\
1572 . \\
5.478\end{array}$ & $\begin{array}{l}195.4 \\
1584 . \\
5.515\end{array}$ & $\begin{array}{l}198.9 \\
1597 \\
5.55 i\end{array}$ & $\begin{array}{l}202.4 \\
1609 . \\
5.586\end{array}$ & $\begin{array}{l}205.8 \\
1621 . \\
5.621\end{array}$ & $\begin{array}{l}209.2 \\
1633 . \\
5.655\end{array}$ & $\begin{array}{l}212.6 \\
1645 . \\
5.688\end{array}$ & $\begin{array}{l}216.0 \\
657 \\
5.721\end{array}$ & $\begin{array}{l}219.4 \\
1669 . \\
5.754\end{array}$ & $\begin{array}{l}222.8 \\
1681 . \\
5.786\end{array}$ & $\begin{array}{l}226.1 \\
1693 . \\
5.817\end{array}$ \\
\hline 900 & $\begin{array}{l}y \\
\text { H } \\
\text { S }\end{array}$ & & $\begin{array}{l}169.2 \\
1567 . \\
5.410\end{array}$ & $\begin{array}{l}172.4 \\
1580 \\
5.447\end{array}$ & $\begin{array}{l}175.6 \\
1593 . \\
5.484\end{array}$ & $\begin{array}{l}178.7 \\
1605 . \\
5.520\end{array}$ & $\begin{array}{l}181.8 \\
1617 . \\
5.555\end{array}$ & $\begin{array}{l}184.9 \\
1630 . \\
5.590^{\circ}\end{array}$ & $\begin{array}{l}188.0 \\
1642 . \\
5.624\end{array}$ & $\begin{array}{l}191.0 \\
\times 54 . \\
5.657\end{array}$ & $\begin{array}{l}194.1 \\
1666 . \\
5.690\end{array}$ & $\begin{array}{l}197.1 \\
1678 . \\
5.722\end{array}$ & $\begin{array}{l}200.1 \\
1691 . \\
5.754\end{array}$ \\
\hline
\end{tabular}


Table 10 (Continued)

Temperature range: 60 to $110^{\circ} \mathrm{C}$

\begin{tabular}{|c|c|c|c|c|c|c|c|c|c|c|c|c|}
\hline P & $\mathbf{T}$ & 60 & 65 & 70 & 75 & 80 & 85 & 90 & 95 & 100 & 105 & 110 \\
\hline 1000 & $\begin{array}{l}7 \\
\mathbf{y} \\
\mathbf{5}\end{array}$ & $\begin{array}{l}151.0 \\
1563 . \\
5.348\end{array}$ & $\begin{array}{l}154.0 \\
1576 . \\
5.386\end{array}$ & $\begin{array}{l}156.9 \\
1588 . \\
5.424\end{array}$ & $\begin{array}{l}159.8 \\
1601 . \\
5.460\end{array}$ & $\begin{array}{l}162.6 \\
1614 . \\
5.496\end{array}$ & $\begin{array}{l}165.5 \\
1626 . \\
5.531\end{array}$ & $\begin{array}{l}168.3 \\
1638 . \\
5.565\end{array}$ & $\begin{array}{l}171.0 \\
1651 . \\
5.599\end{array}$ & $\begin{array}{l}173.8 \\
1663 . \\
5.632\end{array}$ & $\begin{array}{l}176.6 \\
1675 . \\
5.665\end{array}$ & $\begin{array}{l}179.3 \\
1688 . \\
5.657\end{array}$ \\
\hline 1200 & $\begin{array}{l}\nabla \\
u \\
S\end{array}$ & $\begin{array}{l}123.7 \\
15 \leq 3 . \\
5.237\end{array}$ & $\begin{array}{l}126.3 \\
1567 \\
5.277\end{array}$ & $\begin{array}{l}128.8 \\
1580 . \\
5.316\end{array}$ & $\begin{array}{l}131.3 \\
1593 . \\
5.354\end{array}$ & $\begin{array}{l}133.8 \\
1606 . \\
5.391\end{array}$ & $\begin{array}{l}136.2 \\
1619 . \\
5.427\end{array}$ & $\begin{array}{l}138.6 \\
1631 . \\
5,462\end{array}$ & $\begin{array}{l}141.0 \\
1644 \\
5.497\end{array}$ & $\begin{array}{l}143.4 \\
1657 \\
5.531\end{array}$ & $\begin{array}{l}145.7 \\
1669 . \\
5.564\end{array}$ & $\begin{array}{l}148.1 \\
1682 . \\
5.597\end{array}$ \\
\hline 1400 & $\begin{array}{l}\mathbf{I} \\
\mathbf{B} \\
\mathbf{S}\end{array}$ & $\begin{array}{l}104.2 \\
1543 . \\
5.139\end{array}$ & $\begin{array}{l}106.5 \\
1557 . \\
5.181\end{array}$ & $\begin{array}{l}108.8 \\
1571 . \\
5.221\end{array}$ & $\begin{array}{l}111.0 \\
1585 . \\
5.260\end{array}$ & $\begin{array}{l}113.2 \\
1598 . \\
5.298\end{array}$ & $\begin{array}{l}115.3 \\
1611 . \\
5.336\end{array}$ & $\begin{array}{l}117.5 \\
1624 . \\
5.372\end{array}$ & $\begin{array}{l}119.6 \\
1637 . \\
5.408\end{array}$ & $\begin{array}{l}121.6 \\
1650 . \\
5.442\end{array}$ & $\begin{array}{l}123.7 \\
1663 . \\
5.477\end{array}$ & $\begin{array}{l}125.8 \\
1676 . \\
5.510\end{array}$ \\
\hline 1600 & $\begin{array}{l}\nabla \\
\Delta \\
\mathbf{s}\end{array}$ & $\begin{array}{l}89.49 \\
1533 . \\
5.050\end{array}$ & $\begin{array}{l}91.60 \\
1548 . \\
\leq-094\end{array}$ & $\begin{array}{l}93.66 \\
1562 . \\
5.136\end{array}$ & $\begin{array}{l}95.68 \\
1576 . \\
5.176\end{array}$ & $\begin{array}{l}97.67 \\
1590 . \\
5.216\end{array}$ & $\begin{array}{l}99.62 \\
1603 . \\
5.254\end{array}$ & $\begin{array}{l}101.5 \\
1617 . \\
5.292\end{array}$ & $\begin{array}{l}103.4 \\
1630 . \\
5.328\end{array}$ & $\begin{array}{l}105.3 \\
1643 . \\
5.364\end{array}$ & $\begin{array}{l}107.2 \\
1657 . \\
5.399\end{array}$ & $\begin{array}{l}109: 0 \\
1670 . \\
5.433\end{array}$ \\
\hline 1800 & $\begin{array}{l}\nabla \\
8 \\
S\end{array}$ & $\begin{array}{l}77.99 \\
1522 . \\
4.968\end{array}$ & $\begin{array}{l}79.96 \\
1537 . \\
5.013\end{array}$ & $\begin{array}{l}81.88 \\
1552 . \\
5.057\end{array}$ & $\begin{array}{l}83.75 \\
1567 . \\
5.099\end{array}$ & $\begin{array}{l}85.59 \\
1581 . \\
5.140\end{array}$ & $\begin{array}{l}87.38 \\
1595 . \\
5.180\end{array}$ & $\begin{array}{l}89.15 \\
1609 . \\
5.219\end{array}$ & $\begin{array}{l}90.89 \\
1623 . \\
5.256\end{array}$ & $\begin{array}{l}92.61 \\
1637 \\
5.293\end{array}$ & $\begin{array}{l}94.30 \\
1650 . \\
5.328\end{array}$ & $\begin{array}{l}95.57 \\
1663 . \\
5.363\end{array}$ \\
\hline 2000 & $\begin{array}{l}y \\
s\end{array}$ & $\begin{array}{l}68.73 \\
1511 . \\
4.890\end{array}$ & $\begin{array}{l}70.60 \\
1527 . \\
4.938\end{array}$ & $\begin{array}{l}72.41 \\
1543 . \\
4.984\end{array}$ & $\begin{array}{l}74.17 \\
1558 . \\
5.028\end{array}$ & $\begin{array}{l}75.89 \\
1573 . \\
5.070\end{array}$ & $\begin{array}{l}77.57 \\
1587 . \\
5.111\end{array}$ & $\begin{array}{l}79.21 \\
1602 \\
5.15 i\end{array}$ & $\begin{array}{l}80.83 \\
1616 . \\
5.190\end{array}$ & $\begin{array}{l}82.42 \\
1530 \\
5.227\end{array}$ & $\begin{array}{l}83.98 \\
1643 . \\
5.264\end{array}$ & $\begin{array}{l}85.53 \\
1657 \\
5.300\end{array}$ \\
\hline 2200 & $\begin{array}{l}\nabla \\
y \\
5\end{array}$ & $\begin{array}{l}61.10 \\
1499 . \\
4.815\end{array}$ & $\begin{array}{l}62.90 \\
1516 . \\
4.866\end{array}$ & $\begin{array}{l}64.63 \\
1532 . \\
4.914\end{array}$ & $\begin{array}{l}66.30 \\
1548 . \\
4.960\end{array}$ & $\begin{array}{l}67.93 \\
1564 . \\
5.004\end{array}$ & $\begin{array}{l}69.52 \\
1579 \\
5.047\end{array}$ & $\begin{array}{l}71.06 \\
1594 . \\
5.088\end{array}$ & $\begin{array}{l}72.58 \\
1608 . \\
5.128\end{array}$ & $\begin{array}{l}74.07 \\
1623 . \\
5.166\end{array}$ & $\begin{array}{l}75.53 \\
1637 \\
5.204\end{array}$ & $\begin{array}{l}76.97 \\
1651 . \\
5.240\end{array}$ \\
\hline 2400 & $\begin{array}{l}\text { D } \\
\text { s } \\
s\end{array}$ & $\begin{array}{l}54.67 \\
1486 . \\
4.743\end{array}$ & $\begin{array}{l}56.43 \\
1500 . \\
4.796\end{array}$ & $\begin{array}{l}58.10 \\
1522 . \\
4.847\end{array}$ & $\begin{array}{l}59.71 \\
1538 . \\
4.895\end{array}$ & $\begin{array}{l}61.27 \\
1555 . \\
4.942\end{array}$ & $\begin{array}{l}62.78 \\
1570 . \\
4.986\end{array}$ & $\begin{array}{l}64.25 \\
1586 . \\
5.028\end{array}$ & $\begin{array}{l}65.69 \\
1609 \\
5.069\end{array}$ & $\begin{array}{l}67.10 \\
16.5 . \\
5.109\end{array}$ & $\begin{array}{l}68.48 \\
2630 . \\
5.147\end{array}$ & $\begin{array}{l}69.83 \\
1644 . \\
5.185\end{array}$ \\
\hline 2600 & $\begin{array}{l}v \\
8 \\
\text { s }\end{array}$ & $\begin{array}{l}49.17 \\
1473 . \\
4.671\end{array}$ & $\begin{array}{l}5 C .90 \\
1492 . \\
9.729\end{array}$ & $\begin{array}{l}52.53 \\
1510 . \\
4.782\end{array}$ & $\begin{array}{l}54.90 \\
1528 . \\
4.833\end{array}$ & $\begin{array}{l}55.60 \\
1545 . \\
4.881\end{array}$ & $\begin{array}{l}57.06 \\
1561 \\
4.927\end{array}$ & $\begin{array}{l}58.47 \\
1577 \\
4.97 i\end{array}$ & $\begin{array}{l}59.84 \\
1593 . \\
5.014\end{array}$ & $\begin{array}{l}61.18 \\
1608 . \\
5.055\end{array}$ & $\begin{array}{l}62.49 \\
1623 . \\
5.094\end{array}$ & $\begin{array}{l}63.78 \\
1637 . \\
5.133\end{array}$ \\
\hline 2800 & $\begin{array}{l}\dot{y} \\
\dot{y} \\
\mathrm{~s}\end{array}$ & & $\begin{array}{l}96.10 \\
1479 . \\
4.662\end{array}$ & $\begin{array}{l}47.72 \\
1499 . \\
4.719\end{array}$ & $\begin{array}{l}49.25 \\
1517 \\
4.773\end{array}$ & $\begin{array}{l}50.72 \\
1535 . \\
4.823\end{array}$ & $\begin{array}{l}52.13 \\
1552 \\
4.871\end{array}$ & $\begin{array}{l}53.49 \\
1569 . \\
4.917\end{array}$ & $\begin{array}{l}54.81 \\
1585 . \\
4.961\end{array}$ & $\begin{array}{l}56.10 \\
1600 . \\
5.003\end{array}$ & $\begin{array}{l}57.35 \\
1615 . \\
5.049\end{array}$ & $\begin{array}{l}58.58 \\
1630 \\
5.083\end{array}$ \\
\hline 3000 & $\begin{array}{l}\text { V } \\
\text { s } \\
\text { s }\end{array}$ & . & & $\begin{array}{l}43.50 \\
1486 . \\
4.656\end{array}$ & $\begin{array}{l}45.01 \\
1506 . \\
4.713\end{array}$ & $\begin{array}{l}46.46 \\
1525 . \\
4.766\end{array}$ & $\begin{array}{l}47.83 \\
1542 . \\
4.816\end{array}$ & $\begin{array}{l}49.16 \\
1560 . \\
4-864\end{array}$ & $\begin{array}{l}50.44 \\
1576 . \\
4.909\end{array}$ & $\begin{array}{l}51.68 \\
1592 . \\
4.953\end{array}$ & $\begin{array}{l}52.89 \\
1608 . \\
4.995\end{array}$ & $\begin{array}{l}54.07 \\
1624 . \\
5.035\end{array}$ \\
\hline 3200 & $\begin{array}{l}y \\
\text { u } \\
\text { s }\end{array}$ & & & $\begin{array}{l}39.75 \\
1473 . \\
4.594\end{array}$ & $\begin{array}{l}41.27 \\
1494 \\
4.654\end{array}$ & $\begin{array}{l}42.69 \\
1514 . \\
4.7110\end{array}$ & $\begin{array}{l}44.05 \\
1532 . \\
4.763\end{array}$ & $\begin{array}{l}45.35 \\
1550 . \\
4.813\end{array}$ & $\begin{array}{l}46.60 \\
1568 . \\
4.860\end{array}$ & $\begin{array}{l}47.80 \\
1584 . \\
4.905\end{array}$ & $\begin{array}{l}48.97 \\
1609 . \\
4.948\end{array}$ & $\begin{array}{l}50.11 \\
1616 . \\
4.990\end{array}$ \\
\hline 3400 & $\begin{array}{l}\mathrm{B} \\
\mathrm{y}\end{array}$ & & & & $\begin{array}{l}37.92 \\
7482 . \\
4.595\end{array}$ & $\begin{array}{l}39.34 \\
1502 . \\
4.655\end{array}$ & $\begin{array}{l}40.69 \\
1522 . \\
4.710\end{array}$ & $\begin{array}{l}41.96 \\
1541 . \\
4.762\end{array}$ & $\begin{array}{l}43.19 \\
1559 \\
4.811\end{array}$ & $\begin{array}{l}44.36 \\
1576 . \\
4.858\end{array}$ & $\begin{array}{l}45.50 \\
1593 \\
4.903\end{array}$ & $\begin{array}{l}46.60 \\
1609 . \\
4.945\end{array}$ \\
\hline
\end{tabular}


Table 10 (Continued)

Temperature range: 60 to $110^{\circ} \mathrm{C}$

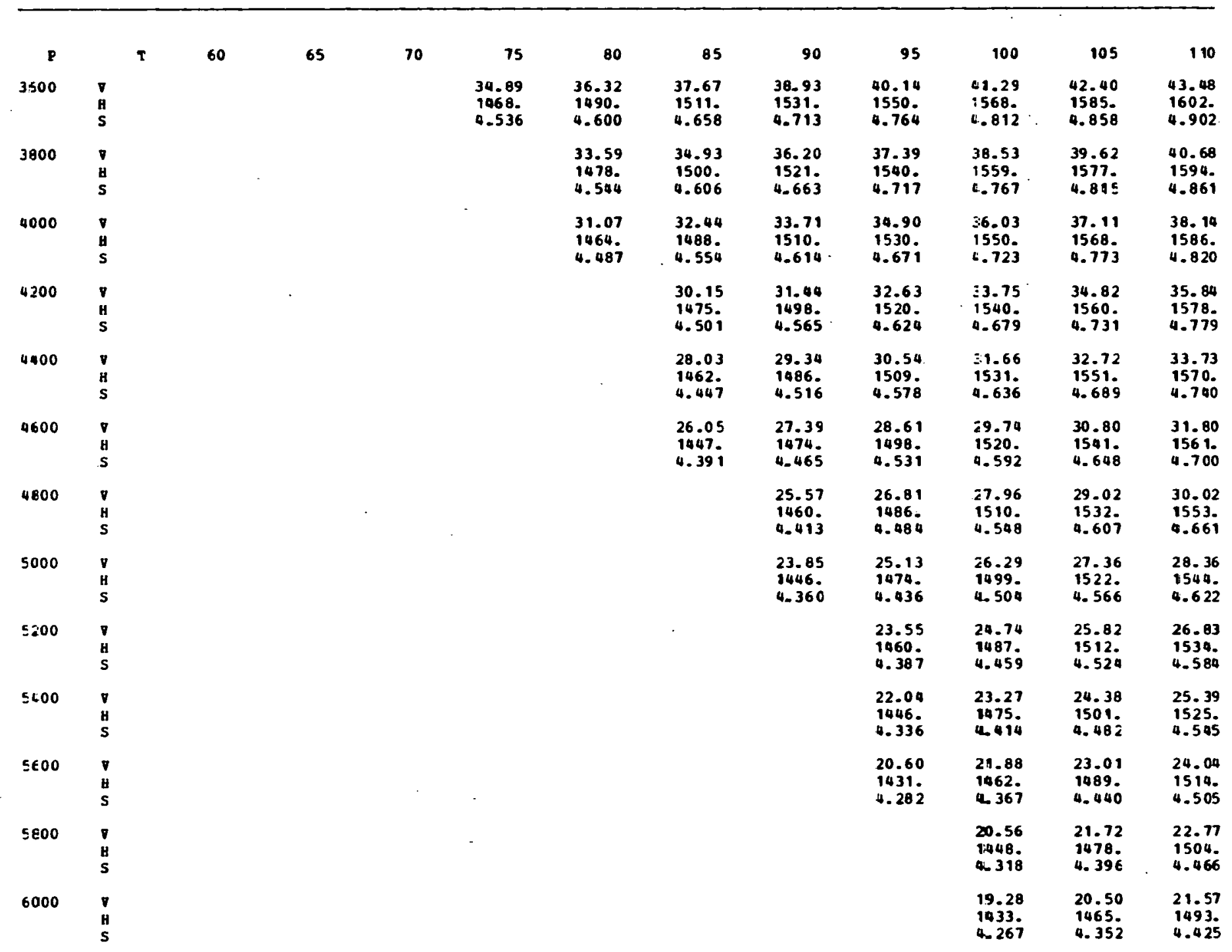


Table 10 (Continued)

Temperature range: 60 to $110^{\circ} \mathrm{C}$

\begin{tabular}{|c|c|c|c|c|c|c|c|c|c|c|c|c|c|}
\hline $\mathbf{P}$ & & $\mathbf{I}$ & 60 & 65 & 70 & 75 & 80 & 85 & 90 & 95 & 100 & 105 & 110 \\
\hline 6200 & 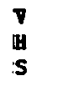 & & & & . & & & & & & $\begin{array}{l}18.04 \\
1416 . \\
4.212\end{array}$ & $\begin{array}{l}19.32 \\
71951 \\
4.306\end{array}$ & $\begin{array}{l}20.43 \\
148 . \\
4.389\end{array}$ \\
\hline 6000 & $\begin{array}{l}\nabla \\
\text { a } \\
\mathbf{s}\end{array}$ & & & $\cdot$ & & ' & & & & & . & $\begin{array}{l}18.18 \\
1437 \\
4.257\end{array}$ & $\begin{array}{l}19.33 \\
1469 . \\
4.342\end{array}$ \\
\hline 6600 & $\begin{array}{l}\mathrm{V} \\
\mathrm{B} \\
\mathrm{S}\end{array}$ & & & & & & & & & & & $\begin{array}{l}17.08 \\
1421 . \\
4.206\end{array}$ & $\begin{array}{l}18.28 \\
1456 \\
4.298\end{array}$ \\
\hline 6800 & $\begin{array}{l}\mathbf{y} \\
\mathrm{u} \\
\mathrm{s}\end{array}$ & & & & & & & & & & . & $\begin{array}{l}15.99 \\
1404 \\
4.151\end{array}$ & $\begin{array}{l}17.27 \\
1442 \\
4.253\end{array}$ \\
\hline 7000 & $\begin{array}{l}\nabla \\
\text { a } \\
\text { S }\end{array}$ & & & & . & & & & & & $\cdot \cdot$ & & $\begin{array}{l}16.28 \\
1427 \\
4.205\end{array}$ \\
\hline 7200 & $\begin{array}{l}\mathbf{v} \\
\mathbf{u} \\
\mathbf{s}\end{array}$ & . & & $\cdot \vdots$ & & , & & - & & & & $\because$ & $\begin{array}{l}15.31 \\
1471 \\
4.154\end{array}$ \\
\hline 7400 & $\begin{array}{l}\text { D } \\
\text { g. } \\
\text { s. }\end{array}$ & & & $\cdots$ & . & & & & . & & & & $\begin{array}{l}14.34 \\
139 . \\
4.099\end{array}$ \\
\hline 7600 & $\begin{array}{l}\text { I } \\
\text { s }\end{array}$ & & & . & & & & & & & & $\therefore$ & \\
\hline 7800 & $\begin{array}{l}y \\
\text { s } \\
\text { s }\end{array}$ & & & $\therefore$ & 3 & & & & 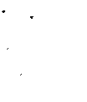 & & & 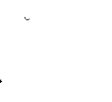 & \\
\hline 8000 & $\begin{array}{l}\nabla \\
\mathbf{B} \\
\mathbf{S}\end{array}$ & & & & & 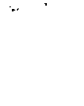 & . & & & $\cdot$ & & $\therefore$ & $\cdot$ \\
\hline 8200 & $\begin{array}{l}\text { I } \\
\text { a } \\
\text { s }\end{array}$ & & . & & & & 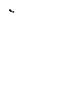 & . & , & & & . ' & \\
\hline 8400 & $\begin{array}{l}\text { D } \\
\text { B } \\
S\end{array}$ & & & & & & - & $\therefore$ & . & & & & \\
\hline 8600 & $\begin{array}{l}8 \\
8 \\
S\end{array}$ & & & & & & & & & & & & \\
\hline
\end{tabular}


Table 10 (Continued)

Temperature range: 115 to $165^{\circ} \mathrm{C}$

\begin{tabular}{|c|c|c|c|c|c|c|c|c|c|c|c|c|}
\hline P & & 115 & .120 & 125 & 130 & 135 & 140 & 145 & 150 & 155 & $160^{\circ}$ & 165 \\
\hline 5 & $\begin{array}{l}V \\
H \\
S\end{array}$ & $\begin{array}{l}5890 . \\
1727 . \\
3.366\end{array}$ & $\begin{array}{c}38380 \\
1738 \\
8.395\end{array}$ & $\begin{array}{c}38840 \\
1750 . \\
8.424\end{array}$ & $\begin{array}{r}3935 \mathrm{~J} \\
176.7 . \\
8.452\end{array}$ & $\begin{array}{r}39840 \\
1772 . \\
8.480\end{array}$ & $\begin{array}{r}40330 . \\
1784 . \\
8.508\end{array}$ & $\begin{array}{c}43820 \\
1795 \\
9.536\end{array}$ & $\begin{array}{r}41310 \\
1807 \\
3.564\end{array}$ & $\begin{array}{l}41790 . \\
1819 \\
8.591\end{array}$ & $\begin{array}{r}42280 \\
1830 \\
8.618\end{array}$ & $\begin{array}{r}42770 . \\
1842 . \\
8.645\end{array}$ \\
\hline 10 & $\begin{array}{l}y \\
H \\
S\end{array}$ & $\begin{array}{c}18940 . \\
1727 . \\
3.028\end{array}$ & $\begin{array}{c}19180 \\
1738 \\
8.057\end{array}$ & $\begin{array}{r}19430 \\
1750 . \\
8.085\end{array}$ & $\begin{array}{c}19670 . \\
1761 . \\
8.114\end{array}$ & $\begin{array}{r}19920 \\
1772= \\
8.142\end{array}$ & $\begin{array}{r}20160 \\
1784 \\
8.170\end{array}$ & $\begin{array}{r}2.3410 \\
1795 \\
3.197\end{array}$ & $\begin{array}{c}20650 \\
1807 \\
3.225\end{array}$ & $\begin{array}{r}20890 . \\
1819 . \\
8.252\end{array}$ & $\begin{array}{r}21140 . \\
1830 . \\
8.279\end{array}$ & $\begin{array}{r}21330 . \\
1842 . \\
8.306\end{array}$ \\
\hline$\leq 0$ & $\begin{array}{l}V \\
H \\
5\end{array}$ & $\begin{array}{l}3780 \\
1726 \\
1.240\end{array}$ & $\begin{array}{l}3830 . \\
1737 . \\
7.269\end{array}$ & $\begin{array}{l}3880 . \\
1749 . \\
7.298\end{array}$ & $\begin{array}{l}3933 . \\
1763 . \\
7.326\end{array}$ & $\begin{array}{l}3980 . \\
1771 . \\
7.355\end{array}$ & $\begin{array}{l}4030 \\
1783 . \\
7.383\end{array}$ & $\begin{array}{l}4080 . \\
1794 . \\
7.410\end{array}$ & $\begin{array}{l}4130 . \\
1806 . \\
7.438\end{array}$ & $\begin{array}{l}4170 \\
1818 . \\
7.465\end{array}$ & $\begin{array}{l}4220 . \\
1829 . \\
7.492\end{array}$ & $\begin{array}{l}4270 . \\
1841 . \\
7.519\end{array}$ \\
\hline 160 & $\begin{array}{l}\nabla \\
H \\
S\end{array}$ & $\begin{array}{l}1890 \\
1725 \\
5.899\end{array}$ & $\begin{array}{l}1910 . \\
1736 . \\
6.928\end{array}$ & $\begin{array}{l}19.40 \\
1747 \\
6.957\end{array}$ & $\begin{array}{l}1963 . \\
1759 . \\
6.936\end{array}$ & $\begin{array}{l}1990 . \\
1770 . \\
7.014\end{array}$ & $\begin{array}{l}2010 \\
1782 \\
7.042\end{array}$ & $\begin{array}{l}2040 . \\
3793 . \\
7.070\end{array}$ & $\begin{array}{l}2060 . \\
1805 . \\
7.098\end{array}$ & $\begin{array}{l}2030 . \\
1.817 \\
7.125\end{array}$ & $\begin{array}{l}2110 . \\
1828 . \\
7.152\end{array}$ & $\begin{array}{l}2130 . \\
1840 . \\
7.179\end{array}$ \\
\hline $1 C 1.33$ & $\begin{array}{l}\mathbf{Y} \\
H \\
S\end{array}$ & $\begin{array}{l}1860 . \\
1725 \\
6.893\end{array}$ & $\begin{array}{l}1890 . \\
1736 . \\
6.922\end{array}$ & $\begin{array}{l}1910 . \\
1747 \\
6.951\end{array}$ & $\begin{array}{l}1943 . \\
1759 . \\
6.989\end{array}$ & $\begin{array}{l}1960 . \\
1770 . \\
7.008\end{array}$ & $\begin{array}{l}1980 \\
1782 . \\
7.036\end{array}$ & $\begin{array}{l}2010 . \\
1793 . \\
7.064\end{array}$ & $\begin{array}{l}2030 . \\
1805 \\
7.091\end{array}$ & $\begin{array}{l}2060 . \\
1817 \\
7.119\end{array}$ & $\begin{array}{l}2080 . \\
1828 . \\
7.146\end{array}$ & $\begin{array}{l}2110 . \\
1840 . \\
7.173\end{array}$ \\
\hline $2 \mathrm{CO}$ & $\begin{array}{l}\mathbf{V} \\
\mathrm{H} \\
\mathrm{S}\end{array}$ & $\begin{array}{l}90 \mathrm{C} .0 \\
1722 . \\
3.556\end{array}$ & $\begin{array}{l}952.5 \\
1733 . \\
6.585\end{array}$ & $\begin{array}{l}964.9 \\
1745 . \\
6.614\end{array}$ & $\begin{array}{l}977.4 \\
1755 \\
6.643\end{array}$ & $\begin{array}{l}989.8 \\
1768 . \\
6.672\end{array}$ & $\begin{array}{l}1000 \\
1780 \\
6.700\end{array}$ & $\begin{array}{l}3010 . \\
1791 . \\
\vdots .728\end{array}$ & $\begin{array}{l}1030 . \\
1803 . \\
5.756\end{array}$ & $\begin{array}{l}1040 . \\
1815 . \\
6.783\end{array}$ & $\begin{array}{l}1050 \\
1826 \\
6.811\end{array}$ & $\begin{array}{l}1060 . \\
1838 . \\
6.338\end{array}$ \\
\hline $3 \mathrm{CO}$ & $\begin{array}{l}V \\
H \\
S\end{array}$ & $\begin{array}{l}524.2 \\
1719 . \\
6.553\end{array}$ & $\begin{array}{l}632.6 \\
1731 . \\
6.383\end{array}$ & $\begin{array}{l}641.0 \\
1742 . \\
6.412\end{array}$ & $\begin{array}{l}649.4 \\
1754 . \\
6.401\end{array}$ & $\begin{array}{l}657.7 \\
1766 . \\
6.469\end{array}$ & $\begin{array}{l}666.0 \\
1777 . \\
6.498\end{array}$ & $\begin{array}{l}574.4 \\
1789 . \\
\vdots .526\end{array}$ & $\begin{array}{l}682.7 \\
1801 . \\
6.554\end{array}$ & $\begin{array}{l}69.1 .0 \\
1812 . \\
6.582\end{array}$ & $\begin{array}{l}699.3 \\
1824 . \\
6.609\end{array}$ & $\begin{array}{l}707.7 \\
1836 . \\
6.536\end{array}$ \\
\hline 400 & $\begin{array}{l}V \\
H \\
S\end{array}$ & $\begin{array}{l}46 t .3 \\
1716 . \\
6.208\end{array}$ & $\begin{array}{l}472.7 \\
1728 . \\
6.237\end{array}$ & $\begin{array}{l}479.0 \\
1740 \\
6.267\end{array}$ & $\begin{array}{l}485.3 \\
1751 . \\
6.2 .76\end{array}$ & $\begin{array}{l}491.7 \\
1763 . \\
6.325\end{array}$ & $\begin{array}{l}498.0 \\
1775 . \\
6.353\end{array}$ & $\begin{array}{l}504.3 \\
1787 \\
\vdots .382\end{array}$ & $\begin{array}{l}510.6 \\
1799 . \\
6.410\end{array}$ & $\begin{array}{l}515.9 \\
1810 . \\
6.438\end{array}$ & $\begin{array}{l}523.1 \\
1822 . \\
6.465\end{array}$ & $\begin{array}{l}529.4 \\
1834 . \\
6.493\end{array}$ \\
\hline 500 & $\begin{array}{l}\text { V } \\
H \\
S\end{array}$ & $\begin{array}{l}371.6 \\
1714 . \\
6.094\end{array}$ & $\begin{array}{l}376.7 \\
1725 . \\
6.124\end{array}$ & $\begin{array}{l}381.8 \\
1737 . \\
6.153\end{array}$ & $\begin{array}{l}386.9 \\
174.9 . \\
6.1183\end{array}$ & $\begin{array}{l}392.0 \\
1761 . \\
6.212\end{array}$ & $\begin{array}{l}397.1 \\
1773 . \\
6.240\end{array}$ & $\begin{array}{l}402.2 \\
1784 . \\
3.269\end{array}$ & $\begin{array}{l}407.3 \\
1796 . \\
6.297\end{array}$ & $\begin{array}{l}41.2 .4 \\
18.08 . \\
6.325\end{array}$ & $\begin{array}{l}417.0 \\
1820 . \\
6.353\end{array}$ & $\begin{array}{l}422.5 \\
1832 . \\
6.380\end{array}$ \\
\hline 600 & $\begin{array}{l}V \\
H \\
S\end{array}$ & $\begin{array}{l}308.4 \\
1711 . \\
5.999\end{array}$ & $\begin{array}{l}312.7 \\
1723 \\
6.030\end{array}$ & $\begin{array}{l}317.0 \\
1735 \\
6.060\end{array}$ & $\begin{array}{l}321.3 \\
1747 . \\
6.099\end{array}$ & $\begin{array}{l}325.6 \\
1758 . \\
6.118\end{array}$ & $\begin{array}{l}329.9 \\
1770 \\
6.147\end{array}$ & $\begin{array}{l}334.2 \\
1782 \\
=176\end{array}$ & $\begin{array}{l}338.4 \\
1794 . \\
3.204\end{array}$ & $\begin{array}{l}342.7 \\
18.06 \\
6.233\end{array}$ & $\begin{array}{l}346.9 \\
1816 . \\
6.260\end{array}$ & $\begin{array}{l}351.2 \\
1830 \\
6.288\end{array}$ \\
\hline 700 & $\begin{array}{l}V \\
H \\
S\end{array}$ & $\begin{array}{l}263.3 \\
1708 . \\
5.919\end{array}$ & $\begin{array}{l}267.0 \\
1720 . \\
5.949\end{array}$ & $\begin{array}{l}270.7 \\
1732 . \\
5.980\end{array}$ & $\begin{array}{l}274.5 \\
174.4 \\
6.009\end{array}$ & $\begin{array}{l}278.2 \\
1756 . \\
6.039\end{array}$ & $\begin{array}{l}281.9 \\
1768 . \\
6.068\end{array}$ & $\begin{array}{l}385.6 \\
1780 . \\
E .097\end{array}$ & $\begin{array}{l}289.2 \\
1792 . \\
5.125\end{array}$ & $\begin{array}{l}29.2 .9 \\
18.04 . \\
6.154\end{array}$ & $\begin{array}{l}296.6 \\
1816 . \\
6.182\end{array}$ & $\begin{array}{l}300.2 \\
1828 . \\
6.209\end{array}$ \\
\hline 800 & $\begin{array}{l}V \\
H \\
S\end{array}$ & $\begin{array}{l}229.4 \\
7 C 5 . \\
5.849\end{array}$ & $\begin{array}{l}232.7 \\
1718 \\
5.879\end{array}$ & $\begin{array}{l}236.0 \\
1730 . \\
5.910\end{array}$ & $\begin{array}{l}239.3 \\
174.2 . \\
5.9 .10\end{array}$ & $\begin{array}{l}242.6 \\
1754 . \\
5.969\end{array}$ & $\begin{array}{l}245.8 \\
1766 . \\
5.999\end{array}$ & $\begin{array}{l}249.1 \\
1778 . \\
E .02 \varepsilon\end{array}$ & $\begin{array}{l}252.3 \\
1790 . \\
3.056\end{array}$ & $\begin{array}{l}255.6 \\
1852 . \\
6.385\end{array}$ & $\begin{array}{l}258.8 \\
1814 . \\
6.113\end{array}$ & $\begin{array}{l}262.0 \\
1826 . \\
6.141\end{array}$ \\
\hline 900 & $\begin{array}{l}V \\
H \\
S\end{array}$ & $\begin{array}{l}503.1 \\
1703 . \\
5.786\end{array}$ & $\begin{array}{l}206.0 \\
1715 \\
5.817\end{array}$ & $\begin{array}{l}209.0 \\
1727 \\
5.847\end{array}$ & $\begin{array}{l}212.0 \\
1737 . \\
5.878\end{array}$ & $\begin{array}{l}214.9 \\
1751 . \\
5.907\end{array}$ & $\begin{array}{l}217.8 \\
1763 . \\
5.937\end{array}$ & $\begin{array}{l}220.7 \\
1775 . \\
\equiv .966\end{array}$ & $\begin{array}{r}223.6 \\
.1788 . \\
5.995\end{array}$ & $\begin{array}{l}225.5 \\
1800 . \\
6.024\end{array}$ & $\begin{array}{l}229.4 \\
1812 . \\
6.052\end{array}$ & $\begin{array}{l}232.3 \\
1824 . \\
6.080\end{array}$ \\
\hline
\end{tabular}


Table 10 (Continued)

Temperature range: 115 to $165^{\circ} \mathrm{C}$

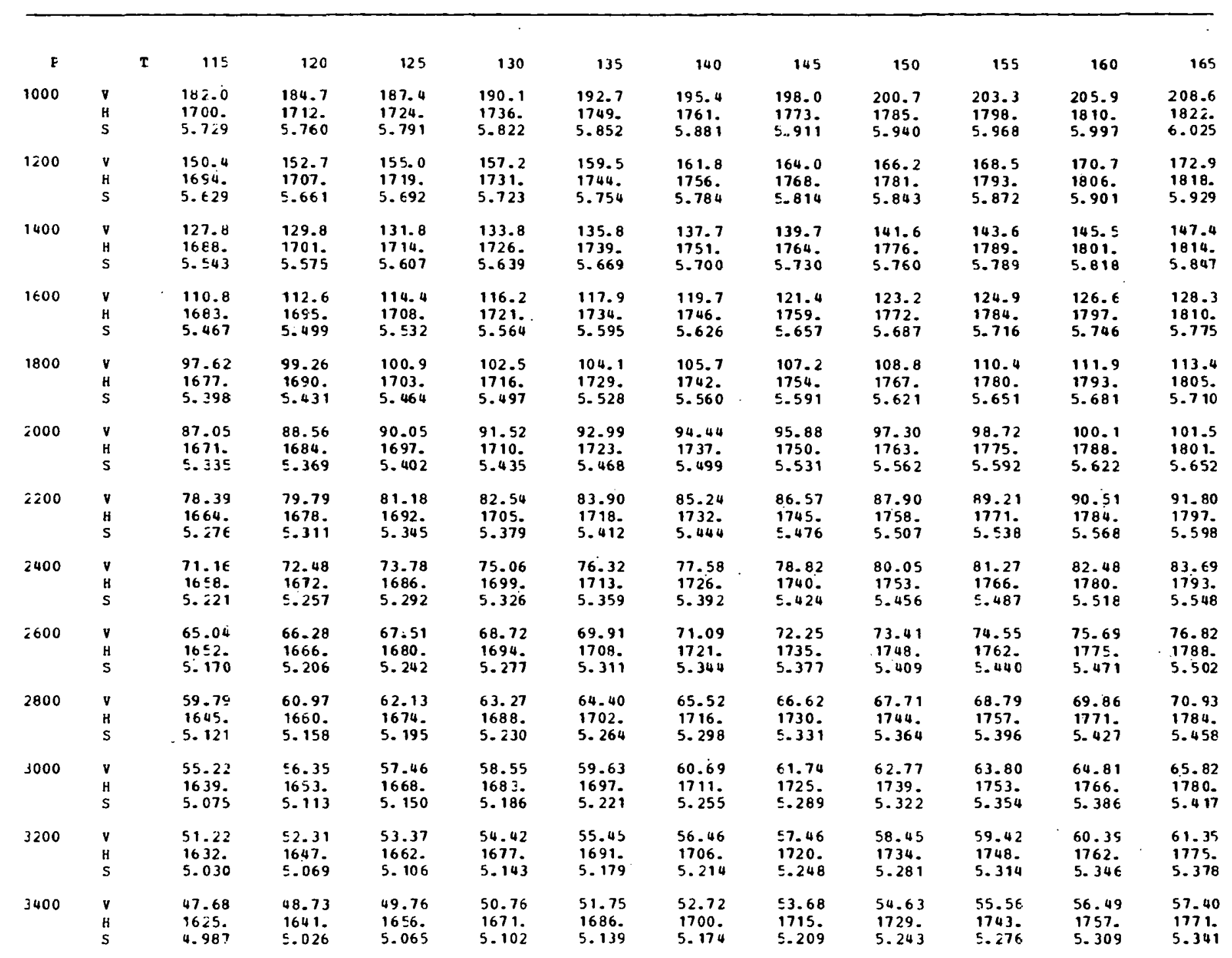


Table 10 (Continued)

Temperature range: 115 to $165^{\circ} \mathrm{C}$

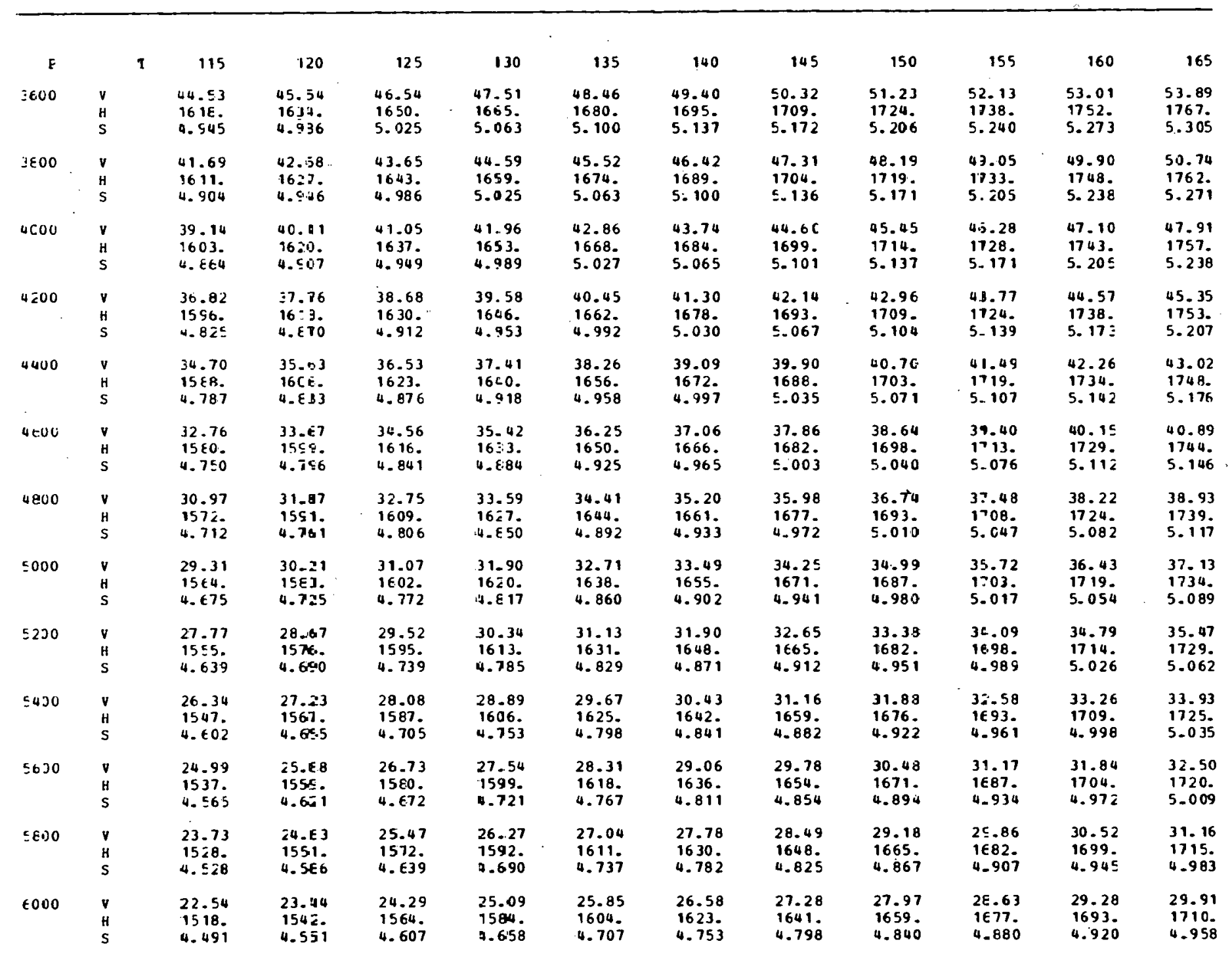


Table 10 (Continued)

Temperature range: 115 to $165^{\circ} \mathrm{C}$

\begin{tabular}{|c|c|c|c|c|c|c|c|c|c|c|c|c|}
\hline P & I & 115 & 120 & 125 & 130 & 135 & 140 & 145 & 150 & 155 & 160 & 165 \\
\hline$\epsilon 200$ & $\begin{array}{l}y \\
\text { E } \\
\text { E. } \\
\text {. }\end{array}$ & $\begin{array}{l}21.42 \\
1508 . \\
4.454\end{array}$ & $\begin{array}{l}22.32 \\
1533 . \\
4.516\end{array}$ & $\begin{array}{l}23.17 \\
1555 . \\
4.574\end{array}$ & $\begin{array}{l}23.97 \\
1577 \\
4.627\end{array}$ & $\begin{array}{l}24.73 \\
1597 \\
4.677\end{array}$ & $\begin{array}{l}25.45 \\
1617 \\
4.725\end{array}$ & $\begin{array}{l}26.15 \\
1 \in 35 \\
4.770\end{array}$ & $\begin{array}{l}26.83 \\
1653 \\
4.813\end{array}$ & $\begin{array}{l}27.48 \\
1671 . \\
4.854\end{array}$ & $\begin{array}{l}28.12 \\
1688 \\
4.894\end{array}$ & $\begin{array}{l}28.74 \\
1705 \\
4.933\end{array}$ \\
\hline$\epsilon 400$ & $\begin{array}{l}\text { Y } \\
\text { H } \\
\text { S }\end{array}$ & $\begin{array}{l}20.35 \\
1457 \\
4.416\end{array}$ & $\begin{array}{l}21.27 \\
1523 . \\
4.489\end{array}$ & $\begin{array}{l}22.12 \\
1547 \\
4.54 i\end{array}$ & $\begin{array}{l}22.92 \\
1569 . \\
4.596\end{array}$ & $\begin{array}{l}23.67 \\
1590 . \\
4.648\end{array}$ & $\begin{array}{l}24.40 \\
16.10: \\
4.696\end{array}$ & $\begin{array}{l}25.05 \\
1629 \\
4.743\end{array}$ & $\begin{array}{l}25.76 \\
1647 \\
4.787\end{array}$ & $\begin{array}{l}26.40 \\
1665 \\
4.829\end{array}$ & $\begin{array}{l}27.03 \\
1683 . \\
4.869\end{array}$ & $\begin{array}{l}27.64 \\
1700 . \\
4.909\end{array}$ \\
\hline 6600 & $\begin{array}{l}7 \\
\text { B } \\
\text { s }\end{array}$ & $\begin{array}{l}19.33 \\
1486 . \\
4.377\end{array}$ & $\begin{array}{l}20.26 \\
1513 . \\
4.446\end{array}$ & $\begin{array}{l}21.12 \\
1538 . \\
4.508\end{array}$ & $\begin{array}{l}21.92 \\
7561 \\
4.565\end{array}$ & $\begin{array}{l}22.68 \\
1582 . \\
4.618\end{array}$ & $\begin{array}{l}23.40 \\
1603 . \\
4.668\end{array}$ & $\begin{array}{l}24.09 \\
1623 \\
4.716\end{array}$ & $\begin{array}{l}24.75 \\
1641 \\
4.761\end{array}$ & $\begin{array}{l}25.39 \\
1660 \\
4.803\end{array}$ & $\begin{array}{l}26.01 \\
1678 \\
4.845\end{array}$ & $\begin{array}{l}26.61 \\
1695 . \\
4.885\end{array}$ \\
\hline 6800 & $\begin{array}{l}\mathrm{Y} \\
\mathrm{H} \\
\mathrm{S}\end{array}$ & $\begin{array}{l}18.35 \\
1875 \\
4.337\end{array}$ & $\begin{array}{l}19.31 \\
1503 . \\
4.410\end{array}$ & $\begin{array}{l}20.18 \\
1529 . \\
4.475\end{array}$ & $\begin{array}{l}20.98 \\
1553 . \\
4.534\end{array}$ & $\begin{array}{l}21.74 \\
1575 . \\
4.589\end{array}$ & $\begin{array}{l}22.45 \\
1596 . \\
4.640\end{array}$ & $\begin{array}{l}23.14 \\
1616 . \\
4.689\end{array}$ & $\begin{array}{l}23.79 \\
1635 . \\
4.735\end{array}$ & $\begin{array}{l}24.43 \\
1654 \\
4.778\end{array}$ & $\begin{array}{l}25.04 \\
1672 \\
4.820\end{array}$ & $\begin{array}{l}25.64 \\
1690 . \\
4.867\end{array}$ \\
\hline 7000 & $\begin{array}{l}y \\
\vdots \\
s\end{array}$ & $\begin{array}{l}17.41 \\
1742 . \\
4.296\end{array}$ & $\begin{array}{l}18.39 \\
1892 \\
4.373\end{array}$ & $\begin{array}{l}19.28 \\
1519 . \\
4.447\end{array}$ & $\begin{array}{l}20.09 \\
1544 . \\
.4 .503\end{array}$ & $\begin{array}{l}20.85 \\
1567 . \\
4.560\end{array}$ & $\begin{array}{l}21.56 \\
1589 . \\
4.612\end{array}$ & $\begin{array}{l}22.24 \\
1609 . \\
4.662\end{array}$ & $\begin{array}{l}22.89 \\
1629 . \\
4.709\end{array}$ & $\begin{array}{l}23.52 \\
1648 \\
4.754\end{array}$ & $\begin{array}{l}24.13 \\
1667 . \\
4.796\end{array}$ & $\begin{array}{l}24.72 \\
1685 . \\
4.837\end{array}$ \\
\hline 7200 & $\begin{array}{l}\text { H } \\
H \\
S\end{array}$ & $\begin{array}{l}16.50 \\
1649 . \\
4.253\end{array}$ & $\begin{array}{l}17.52 \\
1781 \\
4.335\end{array}$ & $\begin{array}{l}18.42 \\
1550 . \\
4.407\end{array}$ & $\begin{array}{l}19.24 \\
1535 \\
4.471\end{array}$ & $\begin{array}{l}20.00 \\
1559 . \\
4.530\end{array}$ & $\begin{array}{l}20.71 \\
1582 . \\
4.585\end{array}$ & $\begin{array}{l}21.39 \\
1603 . \\
4.635\end{array}$ & $\begin{array}{l}22.04 \\
1623 \\
4.683\end{array}$ & $\begin{array}{l}22-66 \\
1642 . \\
4.729\end{array}$ & $\begin{array}{l}23.26 \\
1661 . \\
4.77 j\end{array}$ & $\begin{array}{l}23.85 \\
1679 . \\
4.814\end{array}$ \\
\hline 7400 & $\begin{array}{l}\mathrm{H} \\
\mathrm{H} \\
\mathrm{S}\end{array}$ & $\begin{array}{l}15.62 \\
1435 . \\
4.209\end{array}$ & $\begin{array}{l}16.67 \\
1470 . \\
4.297\end{array}$ & $\begin{array}{l}17.59 \\
1799 . \\
4.373\end{array}$ & $\begin{array}{l}18.42 \\
1526 . \\
4.440\end{array}$ & $\begin{array}{l}19.19 \\
1551 \\
4.501\end{array}$ & $\begin{array}{l}19.91 \\
1574 \\
4.557\end{array}$ & $\begin{array}{l}20.59 \\
1596 . \\
4.609\end{array}$ & $\begin{array}{l}21.23 \\
1617 . \\
4.658\end{array}$ & $\begin{array}{l}21.85 \\
1636 . \\
4.705\end{array}$ & $\begin{array}{l}22.45 \\
1655 \\
4.749\end{array}$ & $\begin{array}{l}23.02 \\
1674 \\
4.792\end{array}$ \\
\hline 7600 & 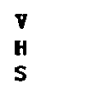 & $\begin{array}{l}14.75 \\
1420 . \\
4.162\end{array}$ & $\begin{array}{l}15.85 \\
1457 \\
4.257\end{array}$ & $\begin{array}{l}16.80 \\
1489 . \\
4.337\end{array}$ & $\begin{array}{c}17.64 \\
1517 . \\
4.408\end{array}$ & $\begin{array}{l}18.42 \\
1543 \\
4.471\end{array}$ & $\begin{array}{l}19.14 \\
1566 . \\
4.529\end{array}$ & $\begin{array}{l}19.82 \\
1589 . \\
4.583\end{array}$ & $\begin{array}{l}20.46 \\
1610 \\
4.633\end{array}$ & $\begin{array}{l}21.08 \\
1630 . \\
4.681\end{array}$ & $\begin{array}{l}21.67 \\
1650 . \\
4.726\end{array}$ & $\begin{array}{l}22.24 \\
1669 . \\
4.769\end{array}$ \\
\hline 7800 & $\begin{array}{l}V \\
H \\
S\end{array}$ & $\begin{array}{l}13.89 \\
1403 \\
4.111\end{array}$ & $\begin{array}{l}15.06 \\
1444 . \\
4.216\end{array}$ & $\begin{array}{l}16.04 \\
1478 . \\
4.301\end{array}$ & $\begin{array}{l}16.90 \\
1507 \\
4.375\end{array}$ & $\begin{array}{l}17.68 \\
1534 . \\
4.4441\end{array}$ & $\begin{array}{l}18.41 \\
1559 \\
4.50 i\end{array}$ & $\begin{array}{l}19.09 \\
1582 . \\
4.556 .\end{array}$ & $\begin{array}{l}19.73 \\
1603 . \\
4.608\end{array}$ & $\begin{array}{l}20.34 \\
1624 \\
4.657\end{array}$ & $\begin{array}{l}20.93 \\
1644 \\
4.703\end{array}$ & $\begin{array}{l}21.50 \\
1663 \\
4.7407\end{array}$ \\
\hline 8000 & $\begin{array}{l}\text { H } \\
\text { H } \\
\text { S }\end{array}$ & $\begin{array}{l}13.02 \\
1385 \\
4.05 E\end{array}$ & $\begin{array}{l}14.28 \\
1430 . \\
4.173\end{array}$ & $\begin{array}{l}15.30 \\
1466 . \\
4.264\end{array}$ & $\begin{array}{l}16.18 \\
1497 \\
4.342\end{array}$ & $\begin{array}{l}16.97 \\
1525: \\
4.411\end{array}$ & $\begin{array}{l}17.70 \\
1551 \\
4.473\end{array}$ & $\begin{array}{l}18.39 \\
1574 \\
4.530\end{array}$ & $\begin{array}{l}19.03 \\
1597 . \\
4.583\end{array}$ & $\begin{array}{l}19.64 \\
1618 . \\
4.633\end{array}$ & $\begin{array}{l}20.22 \\
1638 . \\
4.680\end{array}$ & $\begin{array}{l}20.79 \\
1658 . \\
4.724\end{array}$ \\
\hline 8200 & $\begin{array}{l}\text { V } \\
\text { H } \\
\text { S }\end{array}$ & $\begin{array}{l}12.13 \\
1363 \\
3.995\end{array}$ & $\begin{array}{l}13.51 \\
145 \\
4.127\end{array}$ & $\begin{array}{l}14.58 \\
1454 . \\
4.226\end{array}$ & $\begin{array}{l}15.49 \\
1487 \\
4.308\end{array}$ & $\begin{array}{l}16.30 \\
1516 . \\
4.380\end{array}$ & $\begin{array}{l}17.03 \\
1542 . \\
4.445\end{array}$ & $\begin{array}{l}17.72 \\
1567 \\
4.503\end{array}$ & $\begin{array}{l}18.36 \\
1590 . \\
4.558\end{array}$ & $\begin{array}{l}18.97 \\
1612 . \\
4.609\end{array}$ & $\begin{array}{l}19.55 \\
1632 \\
4.657\end{array}$ & $\begin{array}{l}20.11 \\
1652 \\
4.702\end{array}$ \\
\hline 8400 & $\begin{array}{l}7 \\
H \\
S\end{array}$ & & $\begin{array}{l}12.75 \\
1398 . \\
4.078\end{array}$ & $\begin{array}{l}13.88 \\
1444 . \\
4.186\end{array}$ & $\begin{array}{l}14.82 \\
1476 \\
4.274\end{array}$ & $\begin{array}{l}15.64 \\
1507 . \\
4.349\end{array}$ & $\begin{array}{l}16.39 \\
1534 . \\
4.416\end{array}$ & $\begin{array}{l}17.08 \\
1559 \\
4.477\end{array}$ & $\begin{array}{l}17.72 \\
1583 . \\
4.533\end{array}$ & $\begin{array}{l}18.33 \\
1605 . \\
4.585\end{array}$ & $\begin{array}{l}18.91 \\
1626 \\
4.634\end{array}$ & $\begin{array}{l}19.47 \\
1646 . \\
4.680\end{array}$ \\
\hline 8600 & $\begin{array}{l}V \\
H \\
S\end{array}$ & & $\begin{array}{l}11.97 \\
1379 . \\
4.024\end{array}$ & $\begin{array}{l}13.19 \\
1427 . \\
4.144\end{array}$ & $\begin{array}{l}14.17 \\
1465 . \\
4.238\end{array}$ & $\begin{array}{l}15.01 \\
1497 . \\
4.318\end{array}$ & $\begin{array}{l}15.77 \\
1525 \\
4.387\end{array}$ & $\begin{array}{l}16.46 \\
1552 \\
4.450\end{array}$ & $\begin{array}{l}17.11 \\
1576 \\
4.508\end{array}$ & $\begin{array}{l}17.72 \\
1598 \\
4.561\end{array}$ & $\begin{array}{l}18.30 \\
1620 \\
4.611\end{array}$ & $\begin{array}{l}18.85 \\
1649 \\
4.659\end{array}$ \\
\hline
\end{tabular}


Table 10 (Continued)

Temperature range: 115 to $165^{\circ} \mathrm{C}$

\begin{tabular}{|c|c|c|c|c|c|c|c|c|c|c|c|c|c|}
\hline $\boldsymbol{E}$ & & $I$ & 115 & 120 & 125 & 130 & 135 & 140 & 145 & 150 & 155 & 160 & 165 \\
\hline$\theta \varepsilon 00$ & $\begin{array}{l}\mathbf{1} \\
\mathrm{H} \\
\mathrm{S}\end{array}$ & & . & $\begin{array}{l}11.18 \\
1353 . \\
3.954\end{array}$ & $\begin{array}{l}12.51 \\
1412 . \\
4.100\end{array}$ & $\begin{array}{l}13.53 \\
1453 . \\
4.202\end{array}$ & $\begin{array}{l}14.40 \\
1487 \\
4.285\end{array}$ & $\begin{array}{l}15.17 \\
1516 . \\
4.358\end{array}$ & $\begin{array}{l}15.87 \\
1544 . \\
4.423\end{array}$ & $\begin{array}{l}16.52 \\
1568 . \\
4.483\end{array}$ & $\begin{array}{l}17.13 \\
1592 . \\
4.537\end{array}$ & $\begin{array}{l}17.79 \\
1614 . \\
4.589\end{array}$ & $\begin{array}{l}18.26 \\
1635 \\
4.637\end{array}$ \\
\hline $9 \mathrm{C} 00$ & $\begin{array}{l}: \\
H \\
S\end{array}$ & & & $\begin{array}{l}10.32 \\
1332 \\
3.893\end{array}$ & $\begin{array}{r}11.83 \\
1396 . \\
4.053\end{array}$ & $\begin{array}{l}12.91 \\
1440 . \\
4.164\end{array}$ & $\begin{array}{l}13.81 \\
1476 . \\
4.253\end{array}$ & $\begin{array}{l}14.59 \\
1507 \\
4.329\end{array}$ & $\begin{array}{l}15.30 \\
1535 . \\
4.396\end{array}$ & $\begin{array}{l}15.96 \\
1561 \\
4.457\end{array}$ & $\begin{array}{l}16.57 \\
1585 . \\
4.514\end{array}$ & $\begin{array}{l}17.15 \\
1608 . \\
4.566\end{array}$ & $\begin{array}{l}17.70 \\
1629 . \\
4.615\end{array}$ \\
\hline 9200 & $\begin{array}{l}p \\
\mathrm{t} \\
\mathrm{s}\end{array}$ & & & & $\begin{array}{l}11.14 \\
1378 . \\
4.002\end{array}$ & $\begin{array}{l}12.30 \\
1426 . \\
4.124\end{array}$ & $\begin{array}{l}13.23 \\
1465: \\
4.219\end{array}$ & $\begin{array}{l}14.03 \\
1498 . \\
4.299\end{array}$ & $\begin{array}{l}14.75 \\
1527 . \\
4.369\end{array}$ & $\begin{array}{l}15.41 \\
1553 . \\
4.432\end{array}$ & $\begin{array}{l}16.03 \\
1578 . \\
4.490\end{array}$ & $\begin{array}{l}16.60 \\
1601 . \\
4.544\end{array}$ & $\begin{array}{l}17.15 \\
1623 . \\
4.594\end{array}$ \\
\hline 9400 & $\begin{array}{l}y \\
H \\
\text { S }\end{array}$ & & & & $\begin{array}{l}10.44 \\
1357 . \\
3.945\end{array}$ & $\begin{array}{l}11.70 \\
1412 . \\
4.082\end{array}$ & $\begin{array}{l}12.67 \\
1053 \\
4.184\end{array}$ & $\begin{array}{l}13.49 \\
1488 . \\
4.268\end{array}$ & $\begin{array}{l}14.22 \\
1518 . \\
4.341\end{array}$ & $\begin{array}{l}14.89 \\
1546 \\
4.407\end{array}$ & $\begin{array}{l}15.51 \\
1571 . \\
4.466\end{array}$ & $\begin{array}{l}-16.08 \\
1595 . \\
4.521\end{array}$ & $\begin{array}{l}16.63 \\
1617 . \\
4.573\end{array}$ \\
\hline 96000 & $\begin{array}{l}\mathbf{y} \\
\mathbf{H} \\
\mathbf{S}\end{array}$ & & & & $\begin{array}{l}9.687 \\
1333 . \\
3.879\end{array}$ & $\begin{array}{l}11.09 \\
1396 . \\
4.038\end{array}$ & $\begin{array}{l}12.12 \\
1441 . \\
4.148\end{array}$ & $\begin{array}{l}12.97 \\
1478 \\
4.237\end{array}$ & $\begin{array}{l}13.71 \\
1509 . \\
4.313\end{array}$ & $\begin{array}{l}14.38 \\
1538 \\
4.381\end{array}$ & $\begin{array}{l}15.00 \\
1564 . \\
4.442\end{array}$ & $\begin{array}{l}15.58 \\
15.88 . \\
4: 499\end{array}$ & $\begin{array}{l}16.13 \\
1611 . \\
4.551\end{array}$ \\
\hline $98 \cdot 00$ & $\begin{array}{l}\mathbf{Y} \\
\mathbf{H} \\
\mathbf{S}\end{array}$ & & & & $\begin{array}{l}8.848 \\
1302 . \\
3.797\end{array}$ & $\begin{array}{l}10.49 \\
1379 . \\
3.989\end{array}$ & $\begin{array}{l}11.57 \\
1428 . \\
4.110\end{array}$ & $\begin{array}{l}12.45 \\
1467 \\
4.205\end{array}$ & $\begin{array}{l}13.21 \\
1500 . \\
4.285\end{array}$ & $\begin{array}{l}13.89 \\
1530 . \\
4.355\end{array}$ & $\begin{array}{l}14.52 \\
1557 . \\
4.418\end{array}$ & $\begin{array}{l}15.10 \\
1581 . \\
4.476\end{array}$ & $\begin{array}{l}15.65 \\
1605 \\
4.530\end{array}$ \\
\hline 10000 & $\begin{array}{l}\mathbf{y} \\
\mathbf{H} \\
\mathbf{S}\end{array}$ & & & & & $\begin{array}{l}9.868 \\
.1360 . \\
3.937\end{array}$ & $\begin{array}{l}11.04 \\
1414 \\
4.071\end{array}$ & $\begin{array}{l}11.95 \\
1456 . \\
4.172\end{array}$ & $\begin{array}{l}12.73 \\
1491 . \\
4.256\end{array}$ & $\begin{array}{l}13.42 \\
1521 \\
4.329\end{array}$ & $\begin{array}{l}14.05 \\
1549 . \\
4.394\end{array}$ & $\begin{array}{l}14.64 \\
1575 \\
4.45\end{array}$ & $\begin{array}{l}15.19 \\
1599 . \\
4.508\end{array}$ \\
\hline 10100 & $\begin{array}{l}\mathbf{D} \\
\mathbf{H} \\
\mathbf{S}\end{array}$ & & & & & $\begin{array}{l}9.549 \\
9349 . \\
3.908\end{array}$ & $\begin{array}{l}10.77 \\
1407 \\
4.051\end{array}$ & $\begin{array}{l}11.70 \\
1450 . \\
4.155\end{array}$ & $\begin{array}{l}12.49 \\
1486= \\
4.24 !\end{array}$ & $\begin{array}{l}13.19 \\
1517 . \\
4.316\end{array}$ & $\begin{array}{l}13.82 \\
1545 . \\
4.382\end{array}$ & $\begin{array}{l}14.41 \\
1571 . \\
4.442\end{array}$ & $\begin{array}{l}14.56 \\
1595 . \\
4.498\end{array}$ \\
\hline 10200 & $\begin{array}{l}\mathbf{D} \\
\mathbf{H} \\
\mathbf{S}\end{array}$ & & & & & $\begin{array}{l}9.222 \\
1338 . \\
1.877\end{array}$ & $\begin{array}{l}10.50 \\
1400 \\
4.030\end{array}$ & $\begin{array}{l}11.46 \\
1444 . \\
4.138\end{array}$ & $\begin{array}{l}12.26 \\
1481 \\
4.227\end{array}$ & $\begin{array}{l}12.96 \\
1513 . \\
4.302\end{array}$ & $\begin{array}{l}13.60 \\
1541 . \\
4.370\end{array}$ & $\begin{array}{l}14.19 \\
1568 . \\
4.43 .1\end{array}$ & $\begin{array}{l}14.74 \\
1592 . \\
4.487\end{array}$ \\
\hline 10360 & $\begin{array}{l}\mathbf{V} \\
\mathbf{H} \\
\mathbf{S}\end{array}$ & & & & & $\begin{array}{l}8.882 \\
1325 \\
3.840\end{array}$ & $\begin{array}{l}10.24 \\
1392 . \\
10008\end{array}$ & $\begin{array}{l}11.22 \\
1438 \\
4.121\end{array}$ & $\begin{array}{l}12.03 \\
1476 . \\
4.212\end{array}$ & $\begin{array}{l}12.74 \\
1508 \\
4.289\end{array}$ & $\begin{array}{l}13.38 \\
1538 \\
0.357\end{array}$ & $\begin{array}{l}13.97 \\
1564 . \\
4.419\end{array}$ & $\begin{array}{l}14.52 \\
1589 . \\
4.476\end{array}$ \\
\hline 10400 & $\begin{array}{l}\mathbf{V} \\
\mathrm{H} \\
\mathrm{S}\end{array}$ & & & & & $\begin{array}{l}6.524 \\
1311 \\
3.807\end{array}$ & $\begin{array}{l}.9770 \\
1383 \\
1.985\end{array}$ & $\begin{array}{l}10.98 \\
1432 \\
4.103\end{array}$ & $\begin{array}{l}11.80 \\
1471 . \\
1.196\end{array}$ & $\begin{array}{l}12.52 \\
1504 . \\
4.276\end{array}$ & $\begin{array}{l}13.16 \\
1534 . \\
4.345\end{array}$ & $\begin{array}{l}13.75 \\
1561 \\
4.408\end{array}$ & $\begin{array}{l}14.31 \\
1586 . \\
4.466\end{array}$ \\
\hline $10 \leq 00$ & $\begin{array}{l}V \\
H \\
S\end{array}$ & & & & & $\begin{array}{l}\varepsilon .141 \\
1295 . \\
3.765\end{array}$ & $\begin{array}{l}9.701 \\
1375 \\
3.962\end{array}$ & $\begin{array}{l}10.74 \\
1425 \\
4.085\end{array}$ & $\begin{array}{l}11.58 \\
1465 \\
1.181\end{array}$ & $\begin{array}{l}12.30 \\
1499 \\
7.262\end{array}$ & $\begin{array}{l}12.95 \\
1530 \\
4.333\end{array}$ & $\begin{array}{l}13.54 \\
1557 \\
4.397\end{array}$ & $\begin{array}{l}14.10 \\
1582 . \\
4.455\end{array}$ \\
\hline $1060 \mathrm{~J}$ & $\begin{array}{l}\mathbf{V} \\
H \\
\mathbf{S}\end{array}$ & & & . & & $\begin{array}{l}7.718 \\
1277 \\
3.717\end{array}$ & $\begin{array}{l}9.430 \\
=366 . \\
\equiv .937\end{array}$ & $\begin{array}{l}10.50 \\
1419 . \\
4.066\end{array}$ & $\begin{array}{l}11.35 \\
1460 . \\
1.166\end{array}$ & $\begin{array}{l}12.08 \\
1495 . \\
4.248\end{array}$ & $\begin{array}{l}12.74 \\
1526 . \\
4.320\end{array}$ & $\begin{array}{l}13.33 \\
1553 . \\
4.385\end{array}$ & $\begin{array}{l}13.89 \\
1579 . \\
4.444\end{array}$ \\
\hline
\end{tabular}


Table 10 (Continued)

Temperature range: 115 to $165^{\circ} \mathrm{C}$

\begin{tabular}{|c|c|c|c|c|c|c|c|c|c|c|c|c|c|}
\hline & & & & & & & . & & & & & . & \\
\hline P & & $\mathbf{I}$ & 115 & 120 & 125 & 130 & 135 & 140 & 145 & 150 & 155 & 160 & 165 \\
\hline 10700 & $\begin{array}{l}D \\
H \\
\text { S }\end{array}$ & & & & & $\begin{array}{l}7.222 \\
1253 . \\
3.656\end{array}$ & $\begin{array}{l}9.155 \\
1356 . \\
3.912\end{array}$ & $\begin{array}{l}10.26 \\
1412 . \\
4.048\end{array}$ & $\begin{array}{l}11.13 \\
1455 . \\
4.150\end{array}$ & $\begin{array}{l}11.87 \\
1490 \\
4.234\end{array}$ & $\begin{array}{l}12.53 \\
1521 . \\
4.308\end{array}$ & $\begin{array}{l}13.13 \\
1550 . \\
4.374\end{array}$ & $\begin{array}{l}13.69 \\
1576 . \\
4.433\end{array}$ \\
\hline 10800 & $\begin{array}{l}\mathbf{V} \\
H \\
\mathbf{S}\end{array}$ & & & & & $\begin{array}{l}6.549 \\
1217 \\
3.566\end{array}$ & $\begin{array}{l}8.875 \\
1346: \\
3.884\end{array}$ & $\begin{array}{l}10.03 \\
1405 \\
4.028\end{array}$ & $\begin{array}{l}10.91 \\
1449 . \\
4.134\end{array}$ & $\begin{array}{l}11.66 \\
1485 . \\
4.221\end{array}$ & $\begin{array}{l}12.33 \\
1517 . \\
4.295\end{array}$ & $\begin{array}{l}12.93 \\
1546 . \\
4.363\end{array}$ & $\begin{array}{l}13.48 \\
1572 . \\
4.423\end{array}$ \\
\hline 10900 & $\begin{array}{l}V \\
H \\
S\end{array}$ & & & & & & $\begin{array}{l}8.590 \\
1335 . \\
3.856\end{array}$ & $\begin{array}{l}9.794 \\
1398 . \\
4.008\end{array}$ & $\begin{array}{l}10.70 \\
1443 . \\
4.118\end{array}$ & $\begin{array}{l}11.46 \\
1481 . \\
4.206\end{array}$ & $\begin{array}{l}12.12 \\
1513 \\
4.283\end{array}$ & $\begin{array}{l}12.73 \\
1542 \\
4.351\end{array}$ & $\begin{array}{l}13.29 \\
1569 . \\
4.412\end{array}$ \\
\hline 11000 & $\begin{array}{l}V \\
H \\
S\end{array}$ & & & & & & $\begin{array}{l}8.296 \\
1324 . \\
3.825\end{array}$ & $\begin{array}{l}9.558 \\
1390 . \\
3.988\end{array}$ & $\begin{array}{l}10.48 \\
1437 \\
4.10 i\end{array}$ & $\begin{array}{l}11.25 \\
1476 . \\
4.192\end{array}$ & $\begin{array}{l}11.93 \\
1509 . \\
4.270\end{array}$ & $\begin{array}{l}12.53 \\
1539 . \\
4.335\end{array}$ & $\begin{array}{l}13.09 \\
1566 . \\
4.401\end{array}$ \\
\hline 11100 & $\begin{array}{l}V \\
H \\
S\end{array}$ & & $\cdot$ & & & & $\begin{array}{l}7.992 \\
1.311 . \\
3.792\end{array}$ & $\begin{array}{l}9.323 \\
1382 . \\
3.966\end{array}$ & $\begin{array}{l}10.27 \\
1431 \\
4.084\end{array}$ & $\begin{array}{l}11.05 \\
1471 . \\
4.178\end{array}$ & $\begin{array}{l}11.73 \\
1505 . \\
4.257\end{array}$ & $\begin{array}{l}12.34 \\
1535 . \\
4.327\end{array}$ & $\begin{array}{l}12.90 \\
1562 . \\
4.390\end{array}$ \\
\hline 11200 & $\begin{array}{l}v \\
H \\
S\end{array}$ & & & & & & $\begin{array}{l}7.673 \\
1297 . \\
3.755\end{array}$ & $\begin{array}{l}9.087 \\
1374 . \\
3.944\end{array}$ & $\begin{array}{l}10.06 \\
1425 ; \\
4.067\end{array}$ & $\begin{array}{l}10.85 \\
1466 . \\
4.163\end{array}$ & $\begin{array}{l}11.54 \\
1500 . \\
4.244\end{array}$ & $\begin{array}{l}12.15 \\
1531 . \\
4.316\end{array}$ & $\begin{array}{l}12.71 \\
1559 . \\
4.379\end{array}$ \\
\hline 11300 & $\begin{array}{l}V \\
\text { H } \\
\text { S }\end{array}$ & & & & & & $\begin{array}{l}7.334 \\
1281 . \\
3.715\end{array}$ & $\begin{array}{l}8.849 \\
1366 . \\
3.922\end{array}$ & $\begin{array}{l}5.850 \\
1419 . \\
4.050\end{array}$ & $\begin{array}{l}10.65 \\
1461 \\
4.149\end{array}$ & $\begin{array}{l}11.34 \\
1496 \\
4.231\end{array}$ & $\begin{array}{l}11: 96 \\
1527 \\
4.304\end{array}$ & $\begin{array}{l}12.53 \\
1555 . \\
4.369\end{array}$ \\
\hline 11400 & $\begin{array}{l}V \\
H \\
S\end{array}$ & & & & & & $\begin{array}{l}6.967 \\
1263 . \\
3.668\end{array}$ & $\begin{array}{l}8.610 \\
1357 . \\
3.898\end{array}$ & $\begin{array}{l}9.641 \\
1413 \\
4.032\end{array}$ & $\begin{array}{l}10.46 \\
1455 \\
4.134\end{array}$ & $\begin{array}{l}11.16 \\
1491 . \\
4.218\end{array}$ & $\begin{array}{l}11.78 \\
1523 \\
4.292\end{array}$ & $\begin{array}{l}12.35 \\
1552 . \\
4.358\end{array}$ \\
\hline 11500 & $\begin{array}{l}\nabla \\
H \\
5\end{array}$ & & & . & & 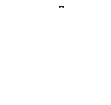 & $\begin{array}{l}6.557 \\
1241 \\
3.613\end{array}$ & $\begin{array}{r}8.369 \\
1348 \\
-3.873\end{array}$ & $\begin{array}{l}9.433 \\
1406 . \\
4.014\end{array}$ & $\begin{array}{l}10.26 \\
1450 \\
4.119\end{array}$ & $\begin{array}{l}10.97 \\
1487 . \\
4.205\end{array}$ & $\begin{array}{l}11.60 \\
1519 . \\
4.280\end{array}$ & $\begin{array}{l}12.17 \\
1548 . \\
4.347\end{array}$ \\
\hline 11600 & $\begin{array}{l}V \\
H \\
S\end{array}$ & & & & & & $\begin{array}{l}6.079 \\
1213 \\
3.544\end{array}$ & $\begin{array}{l}8.124 \\
1338 \\
3.847\end{array}$ & $\begin{array}{l}9.226 \\
1399 \\
3.995\end{array}$ & $\begin{array}{l}10.07 \\
1445 \\
4.103\end{array}$ & $\begin{array}{l}10.7 .9 \\
1482 . \\
4.192\end{array}$ & $\begin{array}{l}11.42 \\
1515 . \\
4.268\end{array}$ & $\begin{array}{l}11.99 \\
1545 . \\
4.336\end{array}$ \\
\hline 11700 & $\begin{array}{l}\mathbf{V} \\
\mathrm{H} \\
\mathrm{S}\end{array}$ & & & & & & $\begin{array}{l}5.480 \\
1175 \\
3.449\end{array}$ & $\begin{array}{l}7.875 \\
1327 \\
3.820\end{array}$ & $\begin{array}{l}9.019 \\
1392 . \\
3.976\end{array}$ & $\begin{array}{l}9.882 \\
1439 \\
4.088\end{array}$ & $\begin{array}{l}10.60 \\
1478 \\
4.179\end{array}$ & $\begin{array}{l}11.24 \\
1511 . \\
4.256\end{array}$ & $\begin{array}{l}11.82 \\
1541 . \\
4.325\end{array}$ \\
\hline 11800 & $\begin{array}{l}V \\
H \\
S\end{array}$ & & & & &. & $\begin{array}{l}4.706 \\
1119 . \\
3.310\end{array}$ & $\begin{array}{l}7.629 \\
.13 .16 . \\
3.791\end{array}$ & $\begin{array}{l}\text { e.813 } \\
1385 \\
3.957\end{array}$ & $\begin{array}{l}9.694 \\
1433 . \\
4.072\end{array}$ & $\begin{array}{l}10.43 \\
1473 . \\
4.165\end{array}$ & $\begin{array}{l}11.07 \\
1507 . \\
4.244\end{array}$ & $\begin{array}{l}11.64 \\
1537 . \\
4.314\end{array}$ \\
\hline 11900 & $\begin{array}{l}\mathbf{V} \\
\mathrm{H} \\
\mathbf{S}\end{array}$ & & & & & & $\begin{array}{l}3.992 \\
1058 . \\
3.160\end{array}$ & $\begin{array}{l}7.361 \\
1304 \\
3.761\end{array}$ & $\begin{array}{l}8.607 \\
1377 . \\
3.936\end{array}$ & $\begin{array}{l}9.507 \\
1428 . \\
0.056\end{array}$ & $\begin{array}{l}10.25 \\
1468 \\
4.151\end{array}$ & $\begin{array}{l}10.85 \\
1503 . \\
4.232\end{array}$ & $\begin{array}{l}11.48 \\
1534 . \\
4.303\end{array}$ \\
\hline
\end{tabular}


Table 10 (Continued)

Temperature range: 115 to $165^{\circ} \mathrm{C}$

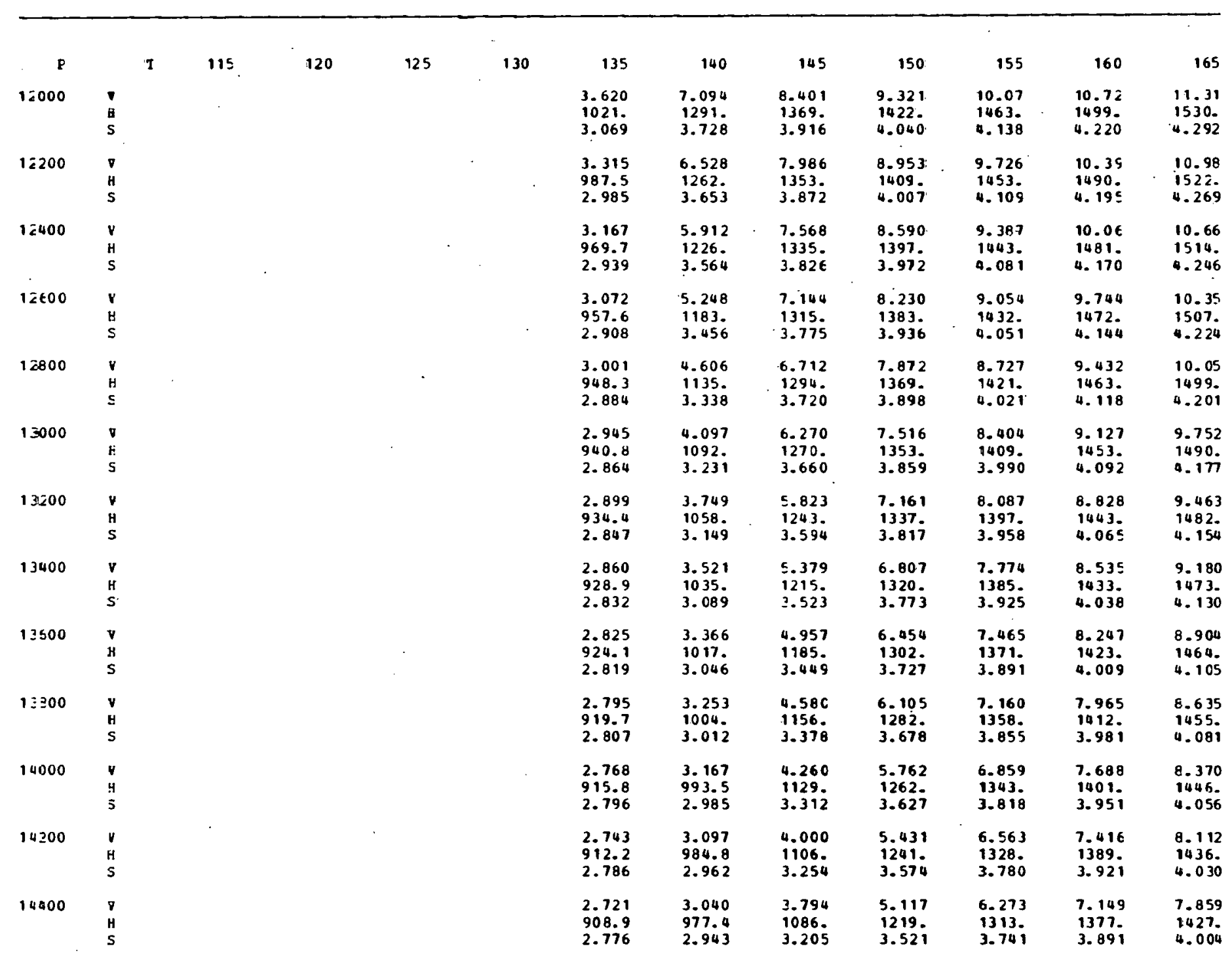


Table 10 (Continued)

Temperature range: 115 to $165^{\circ} \mathrm{C}$

\begin{tabular}{|c|c|c|c|c|c|c|c|c|c|c|c|c|c|}
\hline P & & $T$ & 115 & 120 & 125 & 133 & 135 & 140 & 145 & 150 & 155 & 160 & 165 \\
\hline 14600 & $\begin{array}{l}V \\
H \\
\text { S }\end{array}$ & & & & & & $\begin{array}{l}2.700 \\
905.8 \\
2.767\end{array}$ & $\begin{array}{l}2.991 \\
970.9 \\
2.926\end{array}$ & $\begin{array}{l}3.630 \\
1070 \\
3.163\end{array}$ & $\begin{array}{l}4.827 \\
1198 . \\
3.469\end{array}$ & $\begin{array}{l}5.990 \\
1297 \\
3.701\end{array}$ & $\begin{array}{l}6.888 \\
1365 \\
3.859\end{array}$ & $\begin{array}{l}7.611 \\
1417 . \\
3.978\end{array}$ \\
\hline 14800 & $\begin{array}{l}V \\
H \\
\text { S }\end{array}$ & . & & & & & $\begin{array}{l}2.681 \\
902.9 \\
2.759\end{array}$ & $\begin{array}{l}2.949 \\
965.2 \\
2.911\end{array}$ & $\begin{array}{l}3.499 \\
1056 . \\
3.128\end{array}$ & $\begin{array}{l}4.566 \\
1178 . \\
3.418\end{array}$ & $\begin{array}{l}5.715 \\
1281 . \\
3.660\end{array}$ & $\begin{array}{l}6.632 \\
1352 \\
3.827\end{array}$ & $\begin{array}{l}7.369 \\
1407 . \\
3.951\end{array}$ \\
\hline 15000 & $\begin{array}{l}V \\
H \\
S\end{array}$ & & & & & & $\begin{array}{l}2.663 \\
900.2 \\
2.751\end{array}$ & $\begin{array}{l}2.912 \\
960.2 \\
2.897\end{array}$ & $\begin{array}{l}3.393 \\
1044 . \\
3.098\end{array}$ & $\begin{array}{l}4.336 \\
1159 \\
3.372\end{array}$ & $\begin{array}{l}5.452 \\
1264 . \\
3.618\end{array}$ & $\begin{array}{l}6.382 \\
1340 . \\
3.794\end{array}$ & $\begin{array}{l}7.132 \\
1396 . \\
3.924\end{array}$ \\
\hline 16000 & $\begin{array}{l}\mathbf{V} \\
\text { H } \\
\text { S }\end{array}$ & & & & & & $\begin{array}{l}2.588 \\
888.9 \\
2.717\end{array}$ & $\begin{array}{l}2.774 \\
940.6 \\
2.843\end{array}$ & $\begin{array}{l}3.069 \\
1004 \\
2.996\end{array}$ & $\begin{array}{l}3.585 \\
1088 . \\
3.194\end{array}$ & $\begin{array}{l}4.378 \\
1185 . \\
3.422\end{array}$ & $\begin{array}{l}5.253 \\
1272 \\
3.625\end{array}$ & $\begin{array}{l}6.035 \\
1341 . \\
3.783\end{array}$ \\
\hline 17000 & $\begin{array}{l}\text { V } \\
\text { H } \\
\text { S }\end{array}$ & & & & & & $\begin{array}{l}2.529 \\
879.9 \\
2.689\end{array}$ & $\begin{array}{l}2.680 \\
926.9 \\
2.80 .3\end{array}$ & $\begin{array}{l}2.895 \\
981.2 \\
2.934\end{array}$ & $\begin{array}{l}3.225 \\
1047 \\
3.090\end{array}$ & $\begin{array}{l}3.733 \\
1125 . \\
3.274\end{array}$ & $\begin{array}{l}4.405 \\
1209 \\
3.467\end{array}$ & $\begin{array}{l}5.118 \\
1284 . \\
3.640\end{array}$ \\
\hline 18000 & $\begin{array}{l}\mathbf{v} \\
\mathrm{H} \\
\mathrm{s}\end{array}$ & & & & & & $\begin{array}{l}2.482 \\
872.5 \\
2.664\end{array}$ & $\begin{array}{l}2.610 \\
916.3 \\
2.771\end{array}$ & $\begin{array}{l}2.781 \\
965.0 \\
2.888\end{array}$ & $\begin{array}{l}3.020 \\
1021 \\
3.021\end{array}$ & $\begin{array}{l}3.368 \\
1086 \\
3.174\end{array}$ & $\begin{array}{l}3: 847 \\
1159 \\
3.344\end{array}$ & $\begin{array}{l}4.422 \\
123 i . \\
3.510\end{array}$ \\
\hline 19000 & $\begin{array}{l}\mathbf{y} \\
\text { H } \\
\text { s }\end{array}$ & & & & & & $\begin{array}{l}2.442 \\
866.3 \\
2.643\end{array}$ & $\begin{array}{l}2.554 \\
9077 \\
2.744\end{array}$ & $\begin{array}{l}2.697 \\
952.7 \\
2.852\end{array}$ & $\begin{array}{l}2.886 \\
1003 \\
2.971\end{array}$ & $\begin{array}{l}3.143 \\
1059 . \\
3.104\end{array}$ & $\begin{array}{l}3.492 \\
1122 . \\
3.250\end{array}$ & $\begin{array}{l}3.934 \\
1188 . \\
3.402\end{array}$ \\
\hline 20000 & $\begin{array}{l}V \\
H \\
S\end{array}$ & & & & & & $\begin{array}{l}2.407 \\
860.8 \\
2.624\end{array}$ & $\begin{array}{l}2.507 \\
900.5 \\
2.720\end{array}$ & $\begin{array}{l}2.631 \\
942.9 \\
2.822\end{array}$ & $\begin{array}{l}2.788 \\
989.0 \\
2.932\end{array}$ & $\begin{array}{l}2.992 \\
1040 . \\
3.051\end{array}$ & $\begin{array}{l}3.259 \\
1095 \\
3.180\end{array}$ & $\begin{array}{l}3.599 \\
1155 \\
3.317\end{array}$ \\
\hline$\approx 1000$ & $\begin{array}{l}V \\
H \\
S\end{array}$ & & & & & & $\begin{array}{l}2.377 \\
856.1 \\
2.606\end{array}$ & $\begin{array}{l}2.468 \\
894.4 \\
2.699\end{array}$ & $\begin{array}{l}2.577 \\
934.7 \\
2.797\end{array}$ & $\begin{array}{l}2.711 \\
978: 0 \\
2.899\end{array}$ & $\begin{array}{l}2.881 \\
1025 \\
3.009\end{array}$ & $\begin{array}{l}3.095 \\
1075 \\
3.126\end{array}$ & $\begin{array}{l}3.364 \\
1129 . \\
3.250\end{array}$ \\
\hline 22000 & $\begin{array}{l}\mathbf{v} \\
\mathrm{H} \\
\mathrm{s}\end{array}$ & & & & & & $\begin{array}{l}2.349 \\
851.8 \\
2.590\end{array}$ & $\begin{array}{l}2.433 \\
889.0 \\
2.681\end{array}$ & $\begin{array}{l}2.531 \\
927.8 \\
2.774\end{array}$ & $\begin{array}{l}2.650 \\
968.8 \\
2.871\end{array}$ & $\begin{array}{l}2.794 \\
1013 . \\
2.974\end{array}$ & $\begin{array}{l}2.973 \\
1059 . \\
3.083\end{array}$ & $\begin{array}{l}3.193 \\
1109 . \\
3.196\end{array}$ \\
\hline 23000 & $\begin{array}{l}\mathrm{V} \\
\mathrm{H} \\
\mathrm{S}\end{array}$ & & & & & & $\begin{array}{l}2.325 \\
847.9 \\
2.575\end{array}$ & $\begin{array}{l}2.403 \\
884.3 \\
2.663\end{array}$ & $\begin{array}{l}2.492 \\
921.8 \\
2.753\end{array}$ & $\begin{array}{l}2.598 \\
961.1 \\
2.847\end{array}$ & $\begin{array}{l}2.725 \\
1003 . \\
2.944\end{array}$ & $\begin{array}{l}2.878 \\
.1046 . \\
3.046\end{array}$ & $\begin{array}{l}3.063 \\
1093 . \\
3.152\end{array}$ \\
\hline 24000 & $\begin{array}{l}\mathbf{V} \\
\mathrm{H} \\
\mathrm{S}\end{array}$ & & & & & & $\begin{array}{l}2.302 \\
844.4 \\
2.560\end{array}$ & $\begin{array}{l}2.375 \\
880.1 \\
2.647\end{array}$ & $\begin{array}{l}2.458 \\
916.5 \\
2.735\end{array}$ & $\begin{array}{l}2.554 \\
954.4 \\
2.825\end{array}$ & $\begin{array}{l}2.667 \\
994.0 \\
2.918\end{array}$ & $\begin{array}{l}2.801 \\
1036 \\
3.015\end{array}$ & $\begin{array}{l}2.960 \\
1079 . \\
3.115\end{array}$ \\
\hline 25000 & $\begin{array}{l}\mathbf{V} \\
\mathrm{H} \\
\mathrm{S}\end{array}$ & & & & & & $\begin{array}{l}2.282 \\
849.2 \\
2.547\end{array}$ & $\begin{array}{l}2.351 \\
876.3 \\
2.632\end{array}$ & $\begin{array}{l}2.427 \\
911.8 \\
2.718\end{array}$ & $\begin{array}{l}2.515 \\
948.5 \\
2.805\end{array}$ & $\begin{array}{l}2.617 \\
986.7 \\
2.895\end{array}$ & $\begin{array}{l}2.737 \\
1027 \\
2.987\end{array}$ & $\begin{array}{l}2.877 \\
1068 . \\
3.082\end{array}$ \\
\hline
\end{tabular}


Table :0 (Continued)

Temperature range: 115 to $165^{\circ} \mathrm{C}$

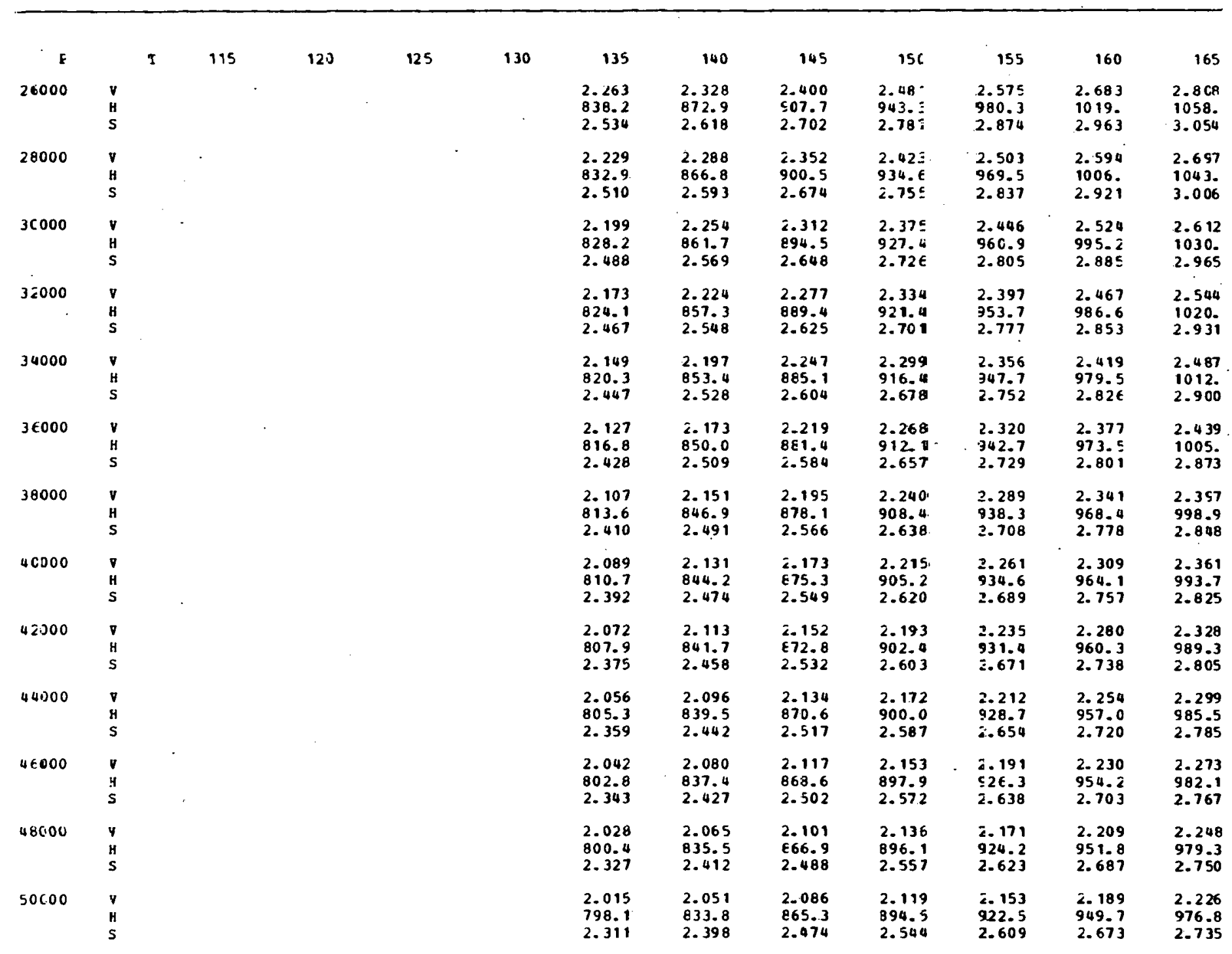


Table 10 (Continued)

Temperature range: 170 to $220^{\circ} \mathrm{C}$

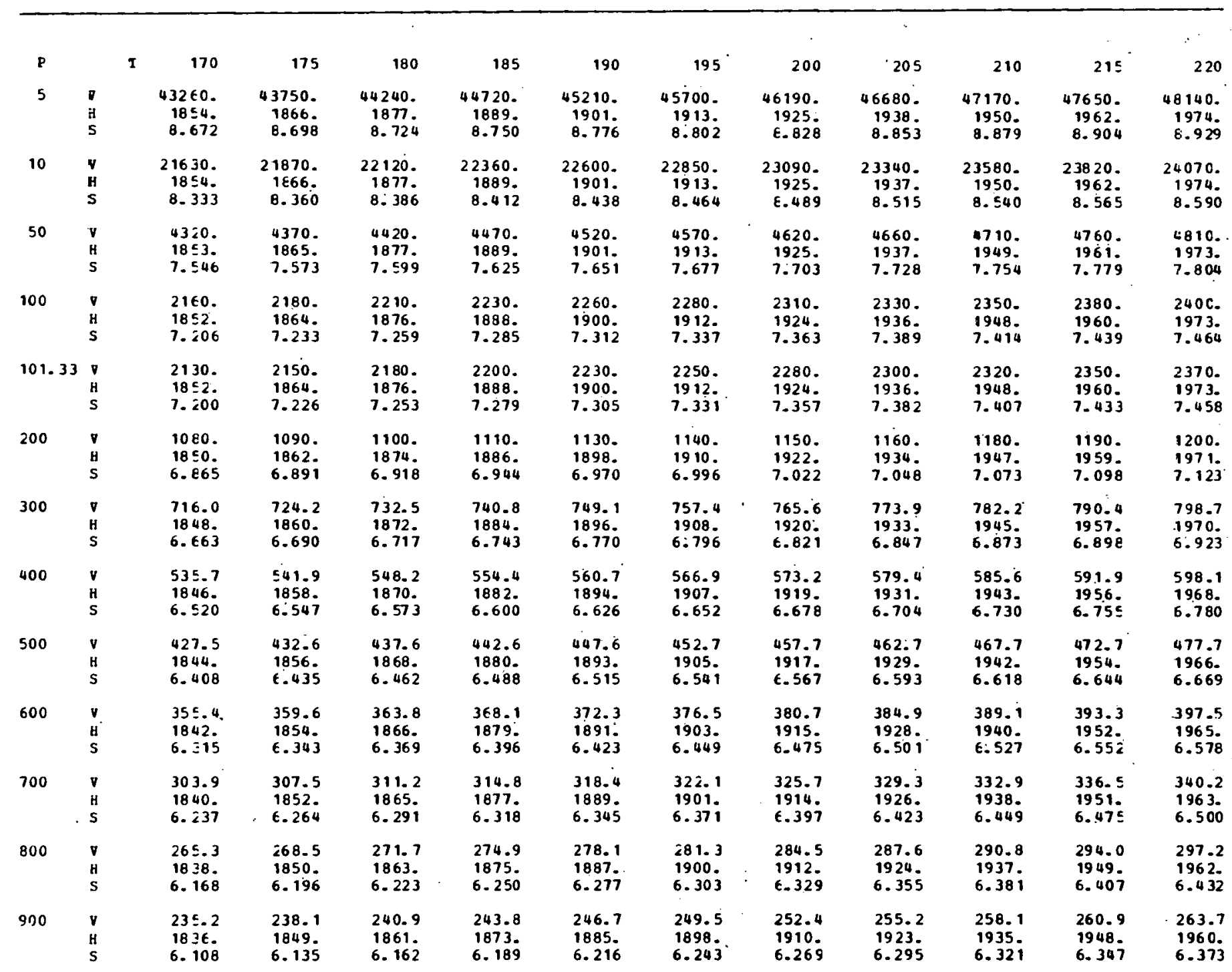


Tæble 10 (Continued)

Temperature range: 170 tc $220^{\circ} \mathrm{C}$

\begin{tabular}{|c|c|c|c|c|c|c|c|c|c|c|c|c|c|}
\hline F & & $\mathbf{T}$ & 170 & 175 & 180 & 185 & 190 & 195 & 200 & 205 & 210 & 215 & 220 \\
\hline 1000 & $\begin{array}{l}\mathbf{H} \\
H \\
\mathbf{S}\end{array}$ & & $\begin{array}{l}211.2 \\
1834 . \\
6.053\end{array}$ & $\begin{array}{l}213.8 \\
1847 . \\
6.080\end{array}$ & $\begin{array}{l}216.4 \\
1859 . \\
6.108\end{array}$ & $\begin{array}{l}2: 9.0 \\
18.71 . \\
6.135\end{array}$ & $\begin{array}{l}221.5 \\
1884 . \\
6.162\end{array}$ & $\begin{array}{l}224.1 \\
1896 . \\
6.189\end{array}$ & $\begin{array}{l}226.7 \\
1909 . \\
6.215\end{array}$ & $\begin{array}{l}229.3 \\
1921 . \\
6.24 i\end{array}$ & $\begin{array}{l}231.8 \\
1934 . \\
6.267\end{array}$ & $\begin{array}{l}234.4 \\
1946 . \\
6.293\end{array}$ & $\begin{array}{l}237.0 \\
1959 . \\
6.319\end{array}$ \\
\hline 1200 & $\begin{array}{l}v \\
H \\
\text { S }\end{array}$ & & $\begin{array}{l}175.1 \\
1830 \\
5.957\end{array}$ & $\begin{array}{l}177.3 \\
1843 . \\
5.985\end{array}$ & $\begin{array}{l}179.5 \\
1855 . \\
6.013\end{array}$ & $\begin{array}{l}\text { IE } 1.7 \\
1 E 68 . \\
6.040\end{array}$ & $\begin{array}{l}183.9 \\
1880 \\
6.067\end{array}$ & $\begin{array}{l}186.0 \\
189.3 . \\
6.094\end{array}$ & $\begin{array}{l}188.2 \\
1905 \\
6.12 i\end{array}$ & $\begin{array}{l}190.4 \\
1918 \\
6.147\end{array}$ & $\begin{array}{l}192.5 \\
1930 . \\
6.173\end{array}$ & $\begin{array}{l}194.7 \\
1943 . \\
6.199\end{array}$ & $\begin{array}{l}196.8 \\
1956 . \\
6.225\end{array}$ \\
\hline 1400 & $\begin{array}{l}v \\
H \\
5\end{array}$ & & $\begin{array}{l}149.3 \\
182 t . \\
5.875\end{array}$ & $\begin{array}{l}151.2 \\
1839 \\
5.903\end{array}$ & $\begin{array}{l}153.1 \\
1851 . \\
5.931\end{array}$ & $\begin{array}{l}1 \leq 5.0 \\
1 E 64 . \\
5.959\end{array}$ & $\begin{array}{l}156.9 \\
1877 . \\
5.986\end{array}$ & $\begin{array}{l}158.8 \\
1889 . \\
6.013\end{array}$ & $\begin{array}{l}160.7 \\
1902 . \\
\epsilon .040\end{array}$ & $\begin{array}{l}162.6 \\
1914 . \\
6.066\end{array}$ & $\begin{array}{l}164.4 \\
1927 \\
6.093\end{array}$ & $\begin{array}{l}166.3 \\
1940 . \\
6.119\end{array}$ & $\begin{array}{l}168.2 \\
1952 . \\
6.145\end{array}$ \\
\hline 1500 & $\begin{array}{l}\mathbf{v} \\
\mathbf{H} \\
\mathbf{S}\end{array}$ & & $\begin{array}{l}130.0 \\
1822 . \\
5.803\end{array}$ & $\begin{array}{l}131.7 \\
1835 . \\
5.832\end{array}$ & $\begin{array}{l}133.4 \\
1848 . \\
5.860\end{array}$ & $\begin{array}{l}1 \equiv 5.1 \\
1 \in 60 . \\
5.888\end{array}$ & $\begin{array}{l}136.7 \\
1873 . \\
5.915\end{array}$ & $\begin{array}{l}138.4 \\
1886 . \\
5.942\end{array}$ & $\begin{array}{l}140.1 \\
1898 . \\
5.969\end{array}$ & $\begin{array}{l}141.7 \\
1911 . \\
5.996\end{array}$ & $\begin{array}{l}143.4 \\
1924 . \\
6.022\end{array}$ & $\begin{array}{l}145.0 \\
1936 . \\
6.049\end{array}$ & $\begin{array}{l}146.7 \\
1949 . \\
6.075\end{array}$ \\
\hline 1.300 & $\begin{array}{l}\nabla \\
\mathbf{H} \\
\mathbf{S}\end{array}$ & & $\begin{array}{l}115.0 \\
1818 . \\
5.739\end{array}$ & $\begin{array}{l}116.5 \\
1831 . \\
5.768\end{array}$ & $\begin{array}{l}118.0 \\
1844 . \\
5.796\end{array}$ & $\begin{array}{l}119.5 \\
1856 . \\
5-824\end{array}$ & $\begin{array}{l}121.0 \\
1869 . \\
5.852\end{array}$ & $\begin{array}{l}122.5 \\
1882 . \\
5.879\end{array}$ & $\begin{array}{l}124.0 \\
1895 . \\
5.906\end{array}$ & $\begin{array}{l}125.5 \\
1908 \\
5.933\end{array}$ & $\begin{array}{l}127.0 \\
1920 . \\
5.960\end{array}$ & $\begin{array}{l}128.5 \\
1933 . \\
5.986\end{array}$ & $\begin{array}{l}130.0 \\
1946 . \\
6.012\end{array}$ \\
\hline 2000 & $\begin{array}{l}v \\
\mathbf{H} \\
5\end{array}$ & & $\begin{array}{l}102.9 \\
1814 . \\
5.681\end{array}$ & $\begin{array}{l}104.3 \\
1827 . \\
5.710\end{array}$ & $\begin{array}{l}10 \leq .7 \\
1840 . \\
5.738\end{array}$ & $\begin{array}{l}107.1 \\
1853 . \\
5.766\end{array}$ & $\begin{array}{l}108.5 \\
1866 . \\
5.794\end{array}$ & $\begin{array}{l}109.8 \\
1878 . \\
5.822\end{array}$ & $\begin{array}{l}111.2 \\
1891 . \\
5.849\end{array}$ & $\begin{array}{l}112.5 \\
1904 . \\
5.876\end{array}$ & $\begin{array}{l}113.9 \\
1917 . \\
5.903\end{array}$ & $\begin{array}{l}115.2 \\
1930 . \\
5.930\end{array}$ & $\begin{array}{l}116.6 \\
1943 . \\
5.956\end{array}$ \\
\hline $2 \approx 00$ & $\begin{array}{l}y \\
y \\
\text { s }\end{array}$ & & $\begin{array}{l}93.09 \\
1810 \\
5.627\end{array}$ & $\begin{array}{l}94.37 \\
1823 . \\
5.656\end{array}$ & $\begin{array}{l}95.64 \\
1836 . \\
5.685\end{array}$ & $\begin{array}{l}96.91 \\
1879 . \\
5.714\end{array}$ & $\begin{array}{l}98.17 \\
1862 . \\
5.742\end{array}$ & $\begin{array}{l}99.43 \\
1875 . \\
5.770\end{array}$ & $\begin{array}{l}100.7 \\
1888 . \\
5.797\end{array}$ & $\begin{array}{l}101.9 \\
1901: \\
5.824\end{array}$ & $\begin{array}{l}103.2 \\
1914 . \\
5.851\end{array}$ & $\begin{array}{l}104.4 \\
1927 . \\
5.879\end{array}$ & $\begin{array}{l}105.6 \\
1940 . \\
5.905\end{array}$ \\
\hline $\begin{array}{c}2400 \\
.\end{array}$ & $\begin{array}{l}y \\
H \\
S\end{array}$ & & $\begin{array}{l}84.88 \\
1806 . \\
5.578\end{array}$ & $\begin{array}{l}86.07 \\
1819 \\
5.607\end{array}$ & $\begin{array}{l}87.25 \\
1332 . \\
5.636\end{array}$ & $\begin{array}{r}88.43 \\
1845 . \\
.5 .565\end{array}$ & $\begin{array}{l}89.60 \\
1858 . \\
5.693\end{array}$ & $\begin{array}{l}90.76 \\
1871 . \\
5.721\end{array}$ & $\begin{array}{l}91.92 \\
1884 \\
5.749\end{array}$ & $\begin{array}{l}93.07 \\
1897 \\
5.777\end{array}$ & $\begin{array}{l}94.22 \\
1910 . \\
5.804\end{array}$ & $\begin{array}{l}95.37 \\
1924 \\
5.831\end{array}$ & $\begin{array}{l}96.51 \\
1937 \\
5.857\end{array}$ \\
\hline $2 \in 00$ & $\begin{array}{l}\mathbf{Y} \\
\mathbf{H} \\
\mathrm{S}\end{array}$ & & $\begin{array}{l}37.94 \\
1802 . \\
5.532\end{array}$ & $\begin{array}{l}79.05 \\
1815 \\
5.561\end{array}$ & $\begin{array}{l}80.15 \\
1828 . \\
5.591\end{array}$ & $\begin{array}{l}81.25 \\
18+1 . \\
5.520\end{array}$ & $\begin{array}{l}82.34 \\
1855 . \\
5.648\end{array}$ & $\begin{array}{l}83.43 \\
1868 . \\
5.677\end{array}$ & $\begin{array}{l}84.51 \\
1881 . \\
5.704\end{array}$ & $\begin{array}{l}85.59 \\
1894 . \\
5.732\end{array}$ & $\begin{array}{l}86.66 \\
1907 \\
5.759\end{array}$ & $\begin{array}{l}87.72 \\
1920 . \\
5.787\end{array}$ & $\begin{array}{l}88.79 \\
1933 . \\
5.813\end{array}$ \\
\hline $2 E 00$ & $\begin{array}{l}y \\
y \\
s\end{array}$ & & $\begin{array}{l}71.98 \\
1798 . \\
5.489\end{array}$ & $\begin{array}{l}73.02 \\
1811 . \\
5.519\end{array}$ & $\begin{array}{l}74.06 \\
1824 \\
5.548\end{array}$ & $\begin{array}{l}75.09 \\
1838 . \\
5.577\end{array}$ & $\begin{array}{l}76.12 \\
1851 . \\
5.606\end{array}$ & $\begin{array}{l}77.14 \\
1864 . \\
5.635\end{array}$ & $\begin{array}{l}78.16 \\
1877 . \\
5.663\end{array}$ & $\begin{array}{l}79.17 \\
1891 \\
5.691\end{array}$ & $\begin{array}{l}80.17 \\
1904 . \\
5.718\end{array}$ & $\begin{array}{l}81.17 \\
1917 \\
5.745\end{array}$ & $\begin{array}{l}82.17 \\
1930 . \\
5.772\end{array}$ \\
\hline 3000 & $\begin{array}{l}V \\
H \\
S\end{array}$ & . & $\begin{array}{l}66.81 \\
1793 . \\
5.448\end{array}$ & $\begin{array}{l}67.80 \\
1807 \\
5.478\end{array}$ & $\begin{array}{l}68.78 \\
1820 \\
5.508\end{array}$ & $\begin{array}{l}69.76 \\
18.14 \\
5.537\end{array}$ & $\begin{array}{l}70.73 \\
1847 . \\
5.566\end{array}$ & $\begin{array}{l}71.69 \\
1860 . \\
5.595\end{array}$ & $\begin{array}{l}72.65 \\
1874 . \\
5.623\end{array}$ & $\begin{array}{l}73.60 \\
1887 \\
5.651\end{array}$ & $\begin{array}{l}74.55 \\
1900 \\
5.679\end{array}$ & $\begin{array}{l}75.45 \\
1914 \\
5.707\end{array}$ & $\begin{array}{l}76.43 \\
1927 . \\
5.734\end{array}$ \\
\hline 3200 & $\begin{array}{l}\mathbf{V} \\
\mathbf{H} \\
\mathbf{S}\end{array}$ & & $\begin{array}{l}62.29 \\
1789 . \\
5.409\end{array}$ & $\begin{array}{l}63.23 \\
1803 . \\
5.440\end{array}$ & $\begin{array}{l}64.16 \\
18.16 . \\
5.470\end{array}$ & $\begin{array}{l}65.09 \\
18.80 . \\
5.499\end{array}$ & $\begin{array}{l}66.01 \\
1843 . \\
5.529\end{array}$ & $\begin{array}{l}66.92 \\
1857 . \\
5.558\end{array}$ & $\begin{array}{l}67.83 \\
1870 \\
5.586\end{array}$ & $\begin{array}{l}68.73 \\
1884 \\
5.614\end{array}$ & $\begin{array}{l}69.63 \\
1897 . \\
5.642\end{array}$ & $\begin{array}{l}70.52 \\
1910 . \\
5.670\end{array}$ & $\begin{array}{l}71.4 .1 \\
1924 \\
5.657\end{array}$ \\
\hline 3430 & $\begin{array}{l}P \\
H \\
S\end{array}$ & & $\begin{array}{l}58.30 \\
17 \varepsilon 5 . \\
5 . \equiv 72\end{array}$ & $\begin{array}{l}59.20 \\
1799 . \\
5.4 \mathrm{C}\end{array}$ & $\begin{array}{l}60.09 \\
1812 \\
5.434\end{array}$ & $\begin{array}{l}60.97 \\
1826 . \\
5.463\end{array}$ & $\begin{array}{l}61.84 \\
1800 . \\
5.493\end{array}$ & $\begin{array}{l}62.71 \\
1853 . \\
5.522\end{array}$ & $\begin{array}{l}63.57 \\
1867 \\
5.551\end{array}$ & $\begin{array}{l}64.43 \\
1880 \\
5.579\end{array}$ & $\begin{array}{l}65.29 \\
1894 \\
5.607\end{array}$ & $\begin{array}{l}66.13 \\
1907 \\
5.635\end{array}$ & $\begin{array}{l}66.98 \\
1921 . \\
5.663\end{array}$ \\
\hline
\end{tabular}


Table 10 (Continued)

Temperature range: 170 to $220^{\circ} \mathrm{C}$

\begin{tabular}{|c|c|c|c|c|c|c|c|c|c|c|c|c|}
\hline$P$ & $\mathbf{I}$ & 170 & 175 & 180 & 185 & 190 & 195 & 200 & 205 & 210 & 215 & 220 \\
\hline$\Xi 600$ & $\begin{array}{l}y \\
\mathbf{H} \\
\mathbf{5}\end{array}$ & $\begin{array}{l}54.75 \\
1781 \\
5.337\end{array}$ & $\begin{array}{l}55.61 \\
: 794 . \\
5.368\end{array}$ & $\begin{array}{l}56.46 \\
1808 . \\
5.399\end{array}$ & $\begin{array}{l}57.30 \\
1822 . \\
5.429\end{array}$ & $\begin{array}{l}58.14 \\
1836 . \\
5.459\end{array}$ & $\begin{array}{l}58.97 \\
1849 \\
5.488\end{array}$ & $\begin{array}{l}59.79 \\
1863 . \\
5.517\end{array}$ & $\begin{array}{l}60.61 \\
1877 \\
5.546\end{array}$ & $\begin{array}{l}61.43 \\
1890 \\
5.574\end{array}$ & $\begin{array}{l}62.23 \\
1904 . \\
5.602\end{array}$ & $\begin{array}{l}63.04 \\
1917 . \\
5.630\end{array}$ \\
\hline 3800 & $\begin{array}{l}\mathbf{D} \\
\mathrm{H} \\
\mathbf{S}\end{array}$ & $\begin{array}{l}51.58 \\
1776 \\
5.303\end{array}$ & $\begin{array}{l}52.40 \\
1790 . \\
5.335\end{array}$ & $\begin{array}{l}53.22 \\
1804 \\
5.366\end{array}$ & $\begin{array}{l}54.02 \\
1818 \\
5.396\end{array}$ & $\begin{array}{l}54.82 \\
1832 . \\
5.426\end{array}$ & $\begin{array}{l}55.62 \\
1846 . \\
5.456\end{array}$ & $\begin{array}{l}56.41 \\
1860 . \\
5.485\end{array}$ & $\begin{array}{l}57.19 \\
1873 . \\
5.514\end{array}$ & $\begin{array}{l}57.97 \\
1887 \\
5.542\end{array}$ & $\begin{array}{l}58.74 \\
1901 \\
5.571\end{array}$ & $\begin{array}{l}59.51 \\
1914 . \\
5.598\end{array}$ \\
\hline 4000 & $\begin{array}{l}\mathbf{Y} \\
H \\
\mathbf{S}\end{array}$ & $\begin{array}{l}48.72 \\
1772 \\
5.271\end{array}$ & $\begin{array}{l}49.51 \\
1786 \\
5.303\end{array}$ & $\begin{array}{l}50.29 \\
1800 \\
5.334\end{array}$ & $\begin{array}{l}51.07 \\
1814 \\
5.365\end{array}$ & $\begin{array}{l}51.84 \\
1828 . \\
5.395\end{array}$ & $\begin{array}{l}52: 60 \\
1842 \\
5.425\end{array}$ & $\begin{array}{l}53.36 \\
1856 \\
5.454\end{array}$ & $\begin{array}{l}54.11 \\
1870 \\
5.483\end{array}$ & $\begin{array}{l}54.86 \\
1883 . \\
5.512\end{array}$ & $\begin{array}{l}55.60 \\
1897 \\
5.540\end{array}$ & $\begin{array}{l}56.34 \\
1911 . \\
5.568\end{array}$ \\
\hline 4200 & $\begin{array}{l}\mathbf{V} \\
\mathrm{H} \\
\mathbf{S}\end{array}$ & $\begin{array}{l}46.13 \\
17 \notin 7 \\
5.239\end{array}$ & $\begin{array}{l}46.89 \\
1782 . \\
5.272\end{array}$ & $\begin{array}{l}47.65 \\
1796 . \\
5.303\end{array}$ & $\begin{array}{l}48.40 \\
1810 . \\
5.334\end{array}$ & $\begin{array}{l}49.14 \\
1824 \\
5.365\end{array}$ & $\begin{array}{l}49.87 \\
1838 . \\
5.395\end{array}$ & $\begin{array}{l}50.60 \\
1852 . \\
5.425\end{array}$ & $\begin{array}{l}51.33 \\
1866 . \\
5.454\end{array}$ & $\begin{array}{l}52.04 \\
1880 \\
5.483\end{array}$ & $\begin{array}{l}52.76 \\
1894 \\
5.511\end{array}$ & $\begin{array}{l}53.47 \\
1908 . \\
5.539\end{array}$ \\
\hline 4400 & $\begin{array}{l}\mathbf{I} \\
\mathbf{H} \\
\mathbf{S}\end{array}$ & $\begin{array}{l}43.77 \\
17 \epsilon 3 . \\
5.209\end{array}$ & $\begin{array}{l}44.51 \\
1778 . \\
5.242\end{array}$ & $\begin{array}{l}45.24 \\
1792 . \\
5.274\end{array}$ & $\begin{array}{l}45.96 \\
1806 . \\
5.305\end{array}$ & $\begin{array}{l}46.68 \\
1820 . \\
5.336\end{array}$ & $\begin{array}{l}47.39 \\
1835 . \\
5.366\end{array}$ & $\begin{array}{l}48.09 \\
1849 . \\
5.396\end{array}$ & $\begin{array}{l}48.79 \\
1863 \\
5.426\end{array}$ & $\begin{array}{l}49.49 \\
1877 \\
5.455\end{array}$ & $\begin{array}{l}50.17 \\
18911 \\
5.483\end{array}$ & $\begin{array}{l}50.86 \\
1904 . \\
5.512\end{array}$ \\
\hline 4600 & $\begin{array}{l}\mathbf{V} \\
\mathrm{H} \\
\mathbf{S}\end{array}$ & $\begin{array}{l}41.62 \\
1759 . \\
5.180\end{array}$ & $\begin{array}{l}42.33 \\
1773 \\
5.213\end{array}$ & $\begin{array}{l}43.04 \\
1788 . \\
5.245\end{array}$ & $\begin{array}{l}43.74 \\
1802 . \\
5.277\end{array}$ & $\begin{array}{l}44.44 \\
18.17 \\
5.308\end{array}$ & $\begin{array}{l}45.12 \\
1831 . \\
5.339\end{array}$ & $\begin{array}{l}45.80 \\
1845 . \\
5.369\end{array}$ & $\begin{array}{l}46.48 \\
1859 . \\
5.398\end{array}$ & $\begin{array}{l}47.15 \\
1873 . \\
5.428\end{array}$ & $\begin{array}{l}47.81 \\
1887 \\
5.456\end{array}$ & $\begin{array}{l}48.47 \\
1901 . \\
5.485\end{array}$ \\
\hline 4800 & $\begin{array}{l}\mathbf{y} \\
\mathbf{H} \\
\mathbf{S}\end{array}$ & $\begin{array}{l}39.64 \\
17 \leqslant 4 \\
5.151\end{array}$ & $\begin{array}{l}40.34 \\
1769 \\
5.185\end{array}$ & $\begin{array}{l}41.03 \\
1784 . \\
5.217\end{array}$ & $\begin{array}{l}41.71 \\
1798 . \\
5.249\end{array}$ & $\begin{array}{l}42.38 \\
1813 . \\
5.281\end{array}$ & $\begin{array}{l}43.04 \\
1827 \\
5.312\end{array}$ & $\begin{array}{l}43.70 \\
1841 \\
5.342\end{array}$ & $\begin{array}{l}44.36 \\
1856 \\
5.372\end{array}$ & $\begin{array}{l}45.0 \mathrm{C} \\
1870 \\
5.401\end{array}$ & $\begin{array}{l}45.65 \\
1884 . \\
5.430\end{array}$ & $\begin{array}{l}46.28 \\
1898 . \\
5.459\end{array}$ \\
\hline$\leq 000$ & $\begin{array}{l}\mathbf{V} \\
\mathbf{H} \\
\mathbf{S}\end{array}$ & $\begin{array}{l}37.82 \\
1749 \\
5.124\end{array}$ & $\begin{array}{l}38.50 \\
1764 \\
5.157\end{array}$ & $\begin{array}{l}39.17 \\
1779 . \\
5.190\end{array}$ & $\begin{array}{l}39.83 \\
1794 . \\
5.223\end{array}$ & $\begin{array}{l}40.48 \\
1809 . \\
5.254\end{array}$ & $\begin{array}{l}41.13 \\
1823 . \\
5.286\end{array}$ & $\begin{array}{l}41.77 \\
1838 \\
5.316\end{array}$ & $\begin{array}{l}42.40 \\
1852 \\
5.346\end{array}$ & $\begin{array}{l}43.03 \\
1866 \\
5.376\end{array}$ & $\begin{array}{l}43.65 \\
1880 \\
5.405\end{array}$ & $\begin{array}{l}44.27 \\
1895 . \\
5.434\end{array}$ \\
\hline$\leq 200$ & $\begin{array}{l}v \\
H \\
5\end{array}$ & $\begin{array}{l}36.14 \\
1745 \\
5.097\end{array}$ & $\begin{array}{l}36.80 \\
1760 \\
5.131\end{array}$ & $\begin{array}{l}37.46 \\
1775 . \\
5.164\end{array}$ & $\begin{array}{l}38.10 \\
1790 \\
5.197\end{array}$ & $\begin{array}{l}38.74 \\
1805 \\
5.229\end{array}$ & $\begin{array}{l}39.36 \\
1819 . \\
5.260\end{array}$ & $\begin{array}{l}39.98 \\
1834 \\
5.291\end{array}$ & $\begin{array}{l}40.60 \\
1848 \\
5.321\end{array}$ & $\begin{array}{l}41.21 \\
1863 \\
5.351\end{array}$ & $\begin{array}{l}41.81 \\
1877 \\
5.381\end{array}$ & $\begin{array}{l}42.41 \\
1891 . \\
5.410\end{array}$ \\
\hline 5400 & $\begin{array}{l}\mathbf{V} \\
\mathbf{H} \\
\mathbf{S}\end{array}$ & $\begin{array}{l}34.59 \\
1740 . \\
5.070\end{array}$ & $\begin{array}{l}35.23 \\
1756- \\
5.105\end{array}$ & $\begin{array}{r}35.87 \\
.1771 \\
5.138\end{array}$ & $\begin{array}{l}36.50 \\
1786 \\
5.172\end{array}$ & $\begin{array}{l}37.11 \\
18.01 . \\
5.204\end{array}$ & $\begin{array}{l}37.73 \\
1816 \\
5.236\end{array}$ & $\begin{array}{l}38.33 \\
1830 \\
5.267\end{array}$ & $\begin{array}{l}38.93 \\
1845 \\
5.297\end{array}$ & $\begin{array}{l}39.52 \\
1859 \\
5.327\end{array}$ & $\begin{array}{l}40.11 \\
1874 \\
5.357\end{array}$ & $\begin{array}{l}40.69 \\
1888 . \\
5.386\end{array}$ \\
\hline 5600 & $\begin{array}{l}\mathbf{v} \\
\mathrm{H} \\
\mathrm{S}\end{array}$ & $\begin{array}{l}33.14 \\
1736 . \\
5.044\end{array}$ & $\begin{array}{l}33.77 \\
1751 . \\
5.079\end{array}$ & $\begin{array}{l}34.39 \\
1767 \\
5.114\end{array}$ & $\begin{array}{l}35.00 \\
1782 . \\
5.147\end{array}$ & $\begin{array}{l}35.61 \\
1797 . \\
5.980\end{array}$ & $\begin{array}{l}36.21 \\
1812 . \\
5.212\end{array}$ & $\begin{array}{l}36.79 \\
1827 \\
5.243\end{array}$ & $\begin{array}{l}37.38 \\
1841 . \\
5.274\end{array}$ & $\begin{array}{l}37.96 \\
1856 \\
5.304\end{array}$ & $\begin{array}{l}38.53 \\
1870 \\
5.334\end{array}$ & $\begin{array}{l}39.09 \\
1885 \\
5.363\end{array}$ \\
\hline 5800 & $\begin{array}{l}\mathbf{V} \\
\mathbf{H} \\
\mathbf{S}\end{array}$ & $\begin{array}{l}31.79 \\
1731 . \\
5.019\end{array}$ & $\begin{array}{l}32.4 i \\
1747 \\
5.055\end{array}$ & $\begin{array}{l}33.02 \\
1762 . \\
5.089\end{array}$ & $\begin{array}{r}33.62 \\
1778 . \\
-5.123\end{array}$ & $\begin{array}{l}34.21 \\
1793 . \\
5.156\end{array}$ & $\begin{array}{l}34.79 \\
1808 \\
5.188\end{array}$ & $\begin{array}{l}35.36 \\
1823 \\
5.220\end{array}$ & $\begin{array}{l}35.93 \\
1838 \\
5.251\end{array}$ & $\begin{array}{l}36.50 \\
1852 . \\
5.281\end{array}$ & $\begin{array}{l}37.05 \\
1867 . \\
5.312\end{array}$ & $\begin{array}{l}37.61 \\
1881 . \\
5.341\end{array}$ \\
\hline 6000 & $\begin{array}{l}\nabla \\
H \\
\text { S }\end{array}$ & $\begin{array}{l}30.5 .3 \\
1726 . \\
4.955\end{array}$ & $\begin{array}{l}31.16 \\
1742 . \\
5.030\end{array}$ & $\begin{array}{l}31.73 \\
1758 . \\
5.065\end{array}$ & $\begin{array}{l}32.32 \\
1773 . \\
5.099\end{array}$ & $\begin{array}{l}32.90 \\
1789 . \\
5.133\end{array}$ & $\begin{array}{l}33.47 \\
180.4 . \\
5.165\end{array}$ & $\begin{array}{l}34.03 \\
1819 \\
5.197\end{array}$ & $\begin{array}{l}34.58 \\
1834 . \\
5.229\end{array}$ & $\begin{array}{l}35.13 \\
1849 . \\
5.259\end{array}$ & $\begin{array}{l}35.68 \\
1863 . \\
5.290\end{array}$ & $\begin{array}{l}36.22 \\
1878 . \\
5.319\end{array}$ \\
\hline
\end{tabular}


Table 10 (Continued)

Temperature range: 170 to $220^{\circ} \mathrm{C}$

\begin{tabular}{|c|c|c|c|c|c|c|c|c|c|c|c|c|}
\hline $\mathbf{P}$ & $I$ & 170 & 175 & 180 & 185 & 190 & 195 & 200 & 205 & 210 & 215 & 220 \\
\hline $6: 00$ & $\begin{array}{l}\mathbf{Y} \\
H \\
S\end{array}$ & $\begin{array}{l}29.35 \\
1721 . \\
4.970\end{array}$ & $\begin{array}{l}29.94 \\
1738 \\
5.007\end{array}$ & $\begin{array}{l}30.53 \\
1754 \\
5.042\end{array}$ & $\begin{array}{l}31.10 \\
1759 . \\
5.376\end{array}$ & $\begin{array}{l}31.67 \\
1785 . \\
5.110\end{array}$ & $\begin{array}{l}32.23 \\
1800 \\
5.143\end{array}$ & $\begin{array}{l}32.78 \\
1815 . \\
5.175\end{array}$ & $\begin{array}{c}33.32 \\
1830 \\
5.207\end{array}$ & $\begin{array}{l}33.86 \\
1845 . \\
5.238\end{array}$ & $\begin{array}{l}34.39 \\
1860 . \\
5.268\end{array}$ & $\begin{array}{l}34.91 \\
1875 . \\
5.298\end{array}$ \\
\hline 6400 & $\begin{array}{l}7 \\
E \\
S\end{array}$ & $\begin{array}{l}28.24 \\
1717 . \\
4.946\end{array}$ & $\begin{array}{l}28.93 \\
1733 . \\
4.933\end{array}$ & $\begin{array}{l}29.40 \\
1749 . \\
5.019\end{array}$ & $\begin{array}{l}29.96 \\
1755 . \\
5.354\end{array}$ & $\begin{array}{l}30.52 \\
1781 . \\
5.088\end{array}$ & $\begin{array}{l}31.06 \\
1796 . \\
5.121\end{array}$ & $\begin{array}{l}31.60 \\
1891 . \\
5.953\end{array}$ & $\begin{array}{l}32.14 \\
1827 \\
5.185\end{array}$ & $\begin{array}{l}32.66 \\
1842 \\
5.217\end{array}$ & $\begin{array}{l}33.18 \\
1857 \\
5.247\end{array}$ & $\begin{array}{l}33.69 \\
1871 . \\
5.278\end{array}$ \\
\hline $6 \in 00$ & $\begin{array}{l}7 \\
\mathrm{Z} \\
\mathrm{S}\end{array}$ & $\begin{array}{l}27.20 \\
1712 . \\
4.923\end{array}$ & $\begin{array}{l}27.77 \\
1723 . \\
4.950\end{array}$ & $\begin{array}{l}28.34 \\
1745 . \\
4.996\end{array}$ & $\begin{array}{l}28.89 \\
1751 . \\
5.332\end{array}$ & $\begin{array}{l}29.44 \\
1777 \\
5.066\end{array}$ & $\begin{array}{l}29.97 \\
1792 . \\
5.099\end{array}$ & $\begin{array}{l}30.50 \\
1808 . \\
5.132\end{array}$ & $\begin{array}{l}31.02 \\
1823 . \\
5.164\end{array}$ & $\begin{array}{l}31.54 \\
1838 . \\
5.196\end{array}$ & $\begin{array}{l}32.05 \\
1853 \\
5.227\end{array}$ & $\begin{array}{l}32.55 \\
1868 . \\
5.257\end{array}$ \\
\hline $6 \varepsilon 00$ & $\begin{array}{l}7 \\
\text { a } \\
\text { s }\end{array}$ & $\begin{array}{l}26.22 \\
17 \mathrm{C7} \\
4.900\end{array}$ & $\begin{array}{l}26.78 \\
1724 . \\
4.938\end{array}$ & $\begin{array}{l}27.34 \\
1740 . \\
4.974\end{array}$ & $\begin{array}{l}27.88 \\
1756 . \\
5.310\end{array}$ & $\begin{array}{l}28.42 \\
1772 . \\
5.044\end{array}$ & $\begin{array}{l}28.94 \\
1788 . \\
5.078\end{array}$ & $\begin{array}{l}29.46 \\
1804 . \\
5.111\end{array}$ & $\begin{array}{l}29.97 \\
1819 . \\
5.144\end{array}$ & $\begin{array}{l}30.48 \\
1834 . \\
5.176\end{array}$ & $\begin{array}{l}30.98 \\
1850 . \\
5.207\end{array}$ & $\begin{array}{l}31.47 \\
1865 . \\
5.238\end{array}$ \\
\hline $7 \times 60$ & $\begin{array}{l}4 \\
y \\
5\end{array}$ & $\begin{array}{l}25.29 \\
1762 \\
4.877\end{array}$ & $\begin{array}{l}25.85 \\
1719 . \\
4.915\end{array}$ & $\begin{array}{l}26.40 \\
1736 \\
4.952\end{array}$ & $\begin{array}{l}26.93 \\
1752 . \\
4.788\end{array}$ & $\begin{array}{l}27.46 \\
1768 \\
5.023\end{array}$ & $\begin{array}{l}27.97 \\
1784 . \\
5.058\end{array}$ & $\begin{array}{l}28.48 \\
1800 \\
5.091\end{array}$ & $\begin{array}{l}28.98 \\
1815 \\
5.124\end{array}$ & $\begin{array}{l}29.48 \\
1831 . \\
5.156\end{array}$ & $\begin{array}{l}29.97 \\
1846 \\
5.187\end{array}$ & $\begin{array}{l}30.45 \\
1861 . \\
5.218\end{array}$ \\
\hline $7: 00$ & $\begin{array}{l}y \\
4 \\
5\end{array}$ & $\begin{array}{l}24.41 \\
1697 \\
4.855\end{array}$ & $\begin{array}{l}24.96 \\
1714 \\
4.893\end{array}$ & $\begin{array}{l}25.50 \\
1731 \\
4.931\end{array}$ & $\begin{array}{l}26.03 \\
1748 . \\
4.767\end{array}$ & $\begin{array}{l}26.55 \\
1764 \\
5.003\end{array}$ & $\begin{array}{l}27.06 \\
1780 \\
5.037\end{array}$ & $\begin{array}{l}27.56 \\
1796 \\
5.07 i\end{array}$ & $\begin{array}{l}28.05 \\
1812 \\
5.104\end{array}$ & $\begin{array}{l}28.54 \\
1827 . \\
5.136\end{array}$ & $\begin{array}{l}29.02 \\
1843 . \\
5.168\end{array}$ & $\begin{array}{l}29.49 \\
1858 . \\
5.199\end{array}$ \\
\hline 7400 & $\begin{array}{l}y \\
\mathbf{y} \\
5\end{array}$ & $\begin{array}{l}23.58 \\
1692 . \\
4.832\end{array}$ & $\begin{array}{l}24.13 \\
1709 \\
4.872\end{array}$ & $\begin{array}{l}24.66 \\
1727 . \\
4.910\end{array}$ & $\begin{array}{l}25.18 \\
17 \div 3 . \\
4.947\end{array}$ & $\begin{array}{l}25.69 \\
1760 . \\
4.982\end{array}$ & $\begin{array}{l}26.19 \\
1776 \\
5.017\end{array}$ & $\begin{array}{l}26.68 \\
1792 \\
5.051\end{array}$ & $\begin{array}{l}27.17 \\
1808 \\
5.085\end{array}$ & $\begin{array}{l}27.64 \\
1824 \\
5.117\end{array}$ & $\begin{array}{l}28.11 \\
1839 . \\
5.149\end{array}$ & $\begin{array}{l}28.58 \\
1854 . \\
5.180\end{array}$ \\
\hline $7 \in 00$ & $\begin{array}{l}1 \\
y \\
5\end{array}$ & $\begin{array}{l}22.79 \\
1687 \\
4.810\end{array}$ & $\begin{array}{l}23.33 \\
9705 . \\
4.850\end{array}$ & $\begin{array}{l}23.86 \\
1722 . \\
4.889\end{array}$ & $\begin{array}{l}24.37 \\
1739 \\
4.926\end{array}$ & $\begin{array}{l}24.87 \\
1756 . \\
4.962\end{array}$ & $\begin{array}{l}25.37 \\
1772 . \\
4.998\end{array}$ & $\begin{array}{l}25.85 \\
1788 . \\
5.032\end{array}$ & $\begin{array}{l}26.33 \\
1804 \\
5.066\end{array}$ & $\begin{array}{l}26.80 \\
1320 \\
5.098\end{array}$ & $\begin{array}{l}27.26 \\
1836 \\
5.131\end{array}$ & $\begin{array}{l}27.72 \\
1851 . \\
5.162\end{array}$ \\
\hline $7 \varepsilon a 0$ & $\begin{array}{l}\text { : } \\
\text { s }\end{array}$ & $\begin{array}{l}22.04 \\
1682 \\
4.789\end{array}$ & $\begin{array}{l}22.58 \\
1705 \\
4.829\end{array}$ & $\begin{array}{l}23.10 \\
1717 . \\
4.868\end{array}$ & $\begin{array}{l}23.60 \\
1735 . \\
4.706\end{array}$ & $\begin{array}{l}24.10 \\
1751 . \\
4.943\end{array}$ & $\begin{array}{l}24.58 \\
1768 . \\
4.978\end{array}$ & $\begin{array}{l}25.06 \\
1784 \\
5.013\end{array}$ & $\begin{array}{l}25.53 \\
1800 \\
5.047\end{array}$ & $\begin{array}{l}25: 99 \\
1816 \\
5.080\end{array}$ & $\begin{array}{l}26.45 \\
1832 . \\
5.112\end{array}$ & $\begin{array}{l}26.90 \\
1848 . \\
5.144\end{array}$ \\
\hline $8 C 00$ & $\begin{array}{l}\mathbf{1} \\
\mathbf{B}\end{array}$ & $\begin{array}{l}21.33 \\
1676 \\
4.767\end{array}$ & $\begin{array}{l}21.96 \\
1695 \\
4.858\end{array}$ & $\begin{array}{l}22.37 \\
1713 . \\
4.848\end{array}$ & $\begin{array}{l}22.87 \\
1730 . \\
4.386\end{array}$ & $\begin{array}{l}23.36 \\
1747 \\
4.923\end{array}$ & $\begin{array}{l}23.84 \\
1764 . \\
4.959\end{array}$ & $\begin{array}{l}24.31 \\
1780 . \\
4.994\end{array}$ & $\begin{array}{l}24.77 \\
1797 \\
5.028\end{array}$ & $\begin{array}{l}25.23 \\
1813 . \\
5.062\end{array}$ & $\begin{array}{l}25.68 \\
1829 \\
5.094\end{array}$ & $\begin{array}{l}26.12 \\
1844 . \\
5.126\end{array}$ \\
\hline$\theta=00$ & $\begin{array}{l}\text { i } \\
\text { s }\end{array}$ & $\begin{array}{l}20.65 \\
1671 . \\
4.746\end{array}$ & $\begin{array}{l}21.17 \\
1699 \\
4.738\end{array}$ & $\begin{array}{l}21.68 \\
1708 . \\
4.828\end{array}$ & $\begin{array}{l}22.18 \\
1726 \\
4.366\end{array}$ & $\begin{array}{l}22.66 \\
1743 . \\
4.904\end{array}$ & $\begin{array}{l}23.13 \\
1760 . \\
4.940\end{array}$ & $\begin{array}{l}23.60 \\
1776 . \\
4.976\end{array}$ & $\begin{array}{l}24.05 \\
1793 . \\
5.010\end{array}$ & $\begin{array}{l}24.50 \\
1809 . \\
5.044\end{array}$ & $\begin{array}{l}24.94 \\
1825 \\
5.077\end{array}$ & $\begin{array}{l}25.38 \\
1841 . \\
5.109\end{array}$ \\
\hline 8400 & $\begin{array}{l}\mathbf{P} \\
\mathbf{8} \\
\mathbf{S}\end{array}$ & $\begin{array}{l}20.00 \\
16 \in \epsilon \\
4.725\end{array}$ & $\begin{array}{l}20.52 \\
1685 \\
4.757\end{array}$ & $\begin{array}{l}21.02 \\
1703 \\
4.808\end{array}$ & $\begin{array}{l}21.51 \\
1721 \\
4.347\end{array}$ & $\begin{array}{l}21.99 \\
1738 \\
4.885\end{array}$ & $\begin{array}{l}22.46 \\
1756 . \\
4.922\end{array}$ & $\begin{array}{l}22.92 \\
1772 \\
4.957\end{array}$ & $\begin{array}{l}23.37 \\
1789 \\
4.992\end{array}$ & $\begin{array}{l}23.81 \\
1805 \\
5.026\end{array}$ & $\begin{array}{l}24.24 \\
1821 . \\
5.059\end{array}$ & $\begin{array}{l}24.67 \\
1837 . \\
5.092\end{array}$ \\
\hline $8 \in 00$ & $\begin{array}{l}\mathbf{r} \\
H \\
5\end{array}$ & $\begin{array}{l}19.38 \\
1661 . \\
4.704\end{array}$ & $\begin{array}{l}19.70 \\
1687 \\
4.747\end{array}$ & $\begin{array}{l}20.40 \\
1698 . \\
4.788\end{array}$ & $\begin{array}{l}20.88 \\
1776 . \\
4.328\end{array}$ & $\begin{array}{l}21.35 \\
1734 . \\
4.866\end{array}$ & $\begin{array}{l}21.82 \\
1751 . \\
4.903\end{array}$ & $\begin{array}{l}22.27 \\
1768 . \\
4.939\end{array}$ & $\begin{array}{l}22.71 \\
1785 \\
4.974\end{array}$ & $\begin{array}{l}23.15 \\
1802 . \\
5.009\end{array}$ & $\begin{array}{l}23.58 \\
1818 . \\
5.042\end{array}$ & $\begin{array}{l}24.00 \\
1834 . \\
5.075\end{array}$ \\
\hline
\end{tabular}


Table 10 (Continued)

Temperature range: 170 to $220^{\circ} \mathrm{C}$

\begin{tabular}{|c|c|c|c|c|c|c|c|c|c|c|c|c|}
\hline P & $\mathrm{T}$ & 170 & 175 & 180 & 185 & 190 & 195 & 200 & 205 & 210 & 215 & 220 \\
\hline 8800 & $\begin{array}{l}y \\
4 \\
5\end{array}$ & $\begin{array}{l}18.79 \\
1655 \\
4.683\end{array}$ & $\begin{array}{l}19.30 \\
1675 \\
4.727\end{array}$ & $\begin{array}{l}19.80 \\
1693 . \\
4.768\end{array}$ & $\begin{array}{l}20.28 \\
1712 . \\
4.809\end{array}$ & $\begin{array}{l}20.74 \\
1730 . \\
4.848\end{array}$ & $\begin{array}{l}21.20 \\
1747 \\
4.885\end{array}$ & $\begin{array}{l}21.65 \\
1764 . \\
4.922 .\end{array}$ & $\begin{array}{l}22.09 \\
1781 \\
4.957\end{array}$ & $\begin{array}{l}22.52 \\
1798 . \\
4.992\end{array}$ & $\begin{array}{l}22.94 \\
1814 \\
5.025\end{array}$ & $\begin{array}{l}23.36 \\
1830 \\
5.058\end{array}$ \\
\hline 9000 & $\begin{array}{l}\mathrm{V} \\
\mathrm{H} \\
\mathrm{S}\end{array}$ & $\begin{array}{l}18.22 \\
16 \leq 0 . \\
4.662\end{array}$ & $\begin{array}{l}18.73 \\
1669 \\
9.707\end{array}$ & $\begin{array}{l}19.22 \\
1689 . \\
4.749\end{array}$ & $\begin{array}{l}19.70 \\
1707 . \\
4.790\end{array}$ & $\begin{array}{l}20.16 \\
1725 . \\
4.829\end{array}$ & $\begin{array}{l}20.61 \\
1743 \\
4.867\end{array}$ & $\begin{array}{l}21.06 \\
1760 . \\
4.904\end{array}$ & $\begin{array}{l}21.49 \\
1777 . \\
4.940\end{array}$ & $\begin{array}{l}21.91 \\
1794 . \\
9.975\end{array}$ & $\begin{array}{l}22.33 \\
1811 . \\
5.009\end{array}$ & $\begin{array}{l}22.74 \\
1827 \\
5.042\end{array}$ \\
\hline 9200 & $\begin{array}{l}\text { D } \\
\text { H } \\
\text { S }\end{array}$ & $\begin{array}{l}17.68 \\
1644 . \\
4.642\end{array}$ & $\begin{array}{l}18.18 \\
1664 \\
4.687\end{array}$ & $\begin{array}{l}18.67 \\
1684 . \\
4.730\end{array}$ & $\begin{array}{l}19.14 \\
1702 . \\
4.771\end{array}$ & $\begin{array}{l}19.60 \\
1721 \\
4.811\end{array}$ & $\begin{array}{l}20.05 \\
1739 . \\
4.849\end{array}$ & $\begin{array}{l}20.49 \\
1756 . \\
4.887\end{array}$ & $\begin{array}{l}20.92 . \\
1773 . \\
4.923\end{array}$ & $\begin{array}{l}21.34 \\
1790 . \\
4.958\end{array}$ & $\begin{array}{l}21.75 \\
1807 \\
4.992\end{array}$ & $\begin{array}{l}22.15 \\
1823 . \\
5.026\end{array}$ \\
\hline 9400 & $\begin{array}{l}D \\
H \\
S\end{array}$ & $\begin{array}{l}17.16 \\
1638 \\
4.62 i\end{array}$ & $\begin{array}{l}17.66 \\
1659 . \\
4.667\end{array}$ & $\begin{array}{l}18.14 \\
1679 \\
4.711\end{array}$ & $\begin{array}{l}18.61 \\
1698 \\
4.753\end{array}$ & $\begin{array}{l}19.07 \\
1716 . \\
4.793\end{array}$ & $\begin{array}{l}19.51 \\
1734 . \\
4.832\end{array}$ & $\begin{array}{l}19.94 \\
1752 . \\
4.869\end{array}$ & $\begin{array}{l}20.37 \\
1769 . \\
4.906\end{array}$ & $\begin{array}{l}20.78 \\
1787 \\
4.941\end{array}$ & $\begin{array}{l}21.19 \\
1803 . \\
4.976\end{array}$ & $\begin{array}{l}21.59 \\
1820 . \\
5.010\end{array}$ \\
\hline 9600 & $\begin{array}{l}\mathbf{V} \\
\mathrm{H} \\
\mathrm{S}\end{array}$ & $\begin{array}{l}16.65 \\
16 \equiv 3 . \\
4.601\end{array}$ & $\begin{array}{l}17.15 \\
1654 \\
4.647\end{array}$ & $\begin{array}{l}17.64 \\
1674 . \\
4.692\end{array}$ & $\begin{array}{l}18.10 \\
1693 . \\
4.734\end{array}$ & $\begin{array}{l}18.55 \\
7712 . \\
4.775\end{array}$ & $\begin{array}{l}18.99 \\
1730 \\
4.814\end{array}$ & $\begin{array}{l}19.42 \\
1748 . \\
4.852\end{array}$ & $\begin{array}{l}19.84 \\
1766 . \\
4.889\end{array}$ & $\begin{array}{l}20.25 \\
1783 . \\
4.925\end{array}$ & $\begin{array}{l}20.65 \\
1800 \\
4.960\end{array}$ & $\begin{array}{l}21.05 \\
1816 . \\
4.994\end{array}$ \\
\hline 9800 & $\begin{array}{l}0 \\
\text { B } \\
5\end{array}$ & $\begin{array}{l}16.17 \\
16.77 \\
4.580\end{array}$ & $\begin{array}{l}16.67 \\
1648 . \\
4.628\end{array}$ & $\begin{array}{l}17.15 \\
1669 \\
4.673\end{array}$ & $\begin{array}{l}17.61 \\
1688 . \\
4.716\end{array}$ & $\begin{array}{l}18.06 \\
1707 . \\
4.757\end{array}$ & $\begin{array}{l}18.50 \\
1726 . \\
4.797\end{array}$ & $\begin{array}{l}18.92 \\
1744 . \\
4.835\end{array}$ & $\begin{array}{l}19.34 \\
1762 . \\
4.873\end{array}$ & $\begin{array}{l}19.74 \\
1779 . \\
4.909\end{array}$ & $\begin{array}{l}20.14 \\
1796 . \\
4.944\end{array}$ & $\begin{array}{l}20.53 \\
1813 . \\
4.978\end{array}$ \\
\hline 10000 & $\begin{array}{l}\nabla \\
\text { H } \\
s\end{array}$ & $\begin{array}{l}15.71 \\
1621 . \\
4.560\end{array}$ & $\begin{array}{l}16.20 \\
1643 . \\
4.608\end{array}$ & $\begin{array}{l}16.68 \\
1663 . \\
4.654\end{array}$ & $\begin{array}{l}17.14 \\
1683 . \\
4.698\end{array}$ & $\begin{array}{l}17.59 \\
1703 . \\
4.740\end{array}$ & $\begin{array}{l}18.02 \\
1721 \\
4.780\end{array}$ & $\begin{array}{l}18.44 \\
1740 . \\
4.815\end{array}$ & $\begin{array}{l}18.85 \\
1758 . \\
4.856\end{array}$ & $\begin{array}{l}19.25 \\
1775 . \\
4.893\end{array}$ & $\begin{array}{l}19.65 \\
1792 . \\
4.928\end{array}$ & $\begin{array}{l}20.03 \\
1809 . \\
4.963\end{array}$ \\
\hline 10100 & $\begin{array}{l}\mathbf{y} \\
\mathrm{H} \\
\mathbf{s}\end{array}$ & $\begin{array}{l}15.48 \\
16 i 8 . \\
4.550\end{array}$ & $\begin{array}{l}15.98 \\
1640 . \\
4.598\end{array}$ & $\begin{array}{l}16.45 \\
1661 . \\
9.645\end{array}$ & $\begin{array}{l}16.91 \\
1681 . \\
4.689\end{array}$ & $\begin{array}{l}17.36 \\
1700 . \\
4.731\end{array}$ & $\begin{array}{l}17.79 \\
1779 . \\
4.771\end{array}$ & $\begin{array}{l}18.21 \\
1738 \\
4.810\end{array}$ & $\begin{array}{l}18.61 \\
1756 . \\
4.848\end{array}$ & $\begin{array}{l}19.02 \\
1773 . \\
4.885\end{array}$ & $\begin{array}{l}19.41 \\
1790 . \\
4.920\end{array}$ & $\begin{array}{l}19.79 \\
1807 . \\
4.955\end{array}$ \\
\hline 10200 & $\begin{array}{l}\mathrm{T} \\
\mathrm{H} \\
\mathrm{S}\end{array}$ & $\begin{array}{l}15.26 \\
1615 . \\
4.539\end{array}$ & $\begin{array}{l}15.75 \\
1637 . \\
4.589\end{array}$ & $\begin{array}{l}16.23 \\
1658 . \\
4.635\end{array}$ & $\begin{array}{l}16.69 \\
1678 . \\
4.680\end{array}$ & $\begin{array}{l}17.13 \\
1699 . \\
4.722\end{array}$ & $\begin{array}{l}17.56 \\
177 . \\
4.763\end{array}$ & $\begin{array}{l}17.98 \\
1735 . \\
4.802\end{array}$ & $\begin{array}{l}18.38 \\
1754 . \\
4.840\end{array}$ & $\begin{array}{l}18.78 \\
1771 \\
4.877\end{array}$ & $\begin{array}{l}19.17 \\
1789 . \\
4.913\end{array}$ & $\begin{array}{l}19.56 \\
1806 . \\
4.947\end{array}$ \\
\hline 10300 & $\begin{array}{l}\mathbf{v} \\
\mathrm{H} \\
\mathrm{s}\end{array}$ & $\begin{array}{l}15.04 \\
1612 . \\
4.529\end{array}$ & $\begin{array}{l}15.54 \\
1634 . \\
4.579\end{array}$ & $\begin{array}{l}16.01 \\
1656 . \\
4.626\end{array}$ & $\begin{array}{l}16.47 \\
1676 \\
4.677\end{array}$ & $\begin{array}{l}16.91 \\
1696 \\
4.713\end{array}$ & $\begin{array}{l}17.34 \\
175.5 \\
4.754\end{array}$ & $\begin{array}{l}17.75 \\
1733 . \\
4.794\end{array}$ & $\begin{array}{l}18.16 \\
1752 . \\
4.832\end{array}$ & $\begin{array}{l}18.55 \\
1769 . \\
4.869\end{array}$ & $\begin{array}{l}18.94 \\
187 . \\
4.905\end{array}$ & $\begin{array}{l}19.32 \\
1804 \\
4.940\end{array}$ \\
\hline 10400 & $\begin{array}{l}\mathrm{v} \\
\mathrm{H} \\
\mathrm{s}\end{array}$ & $\begin{array}{l}14.83 \\
1609 . \\
4.519\end{array}$ & $\begin{array}{l}15.32 \\
1632 . \\
4.569\end{array}$ & $\begin{array}{l}15.80 \\
1653 . \\
4.617\end{array}$ & $\begin{array}{l}16.25 \\
1674 . \\
4.662\end{array}$ & $\begin{array}{l}16.69 \\
1693 . \\
4.705\end{array}$ & $\begin{array}{l}17.12 \\
1773 . \\
4.746\end{array}$ & $\begin{array}{l}17.53 \\
1731 . \\
4.786\end{array}$ & $\begin{array}{l}17.93 \\
1750 . \\
4.824\end{array}$ & $\begin{array}{l}18.33 \\
1767 . \\
4.861\end{array}$ & $\begin{array}{l}18.72 \\
1785 . \\
4.897\end{array}$ & $\begin{array}{l}19.09 \\
1802 \\
4.932\end{array}$ \\
\hline $10 \leq 00$ & $\begin{array}{l}\mathbf{y} \\
\mathrm{H} \\
\mathrm{S}\end{array}$ & $\begin{array}{l}14.62 \\
1606 . \\
4.509\end{array}$ & $\begin{array}{l}15.11 \\
1629 . \\
4.560\end{array}$ & $\begin{array}{l}15.58 \\
1650 \\
4.607\end{array}$ & $\begin{array}{l}16.04 \\
1671 . \\
4.653\end{array}$ & $\begin{array}{l}16-48 \\
1691 . \\
4.696\end{array}$ & $\begin{array}{l}16.90 \\
170 . \\
4.738\end{array}$ & $\begin{array}{l}17.31 \\
1729 . \\
4.778\end{array}$ & $\begin{array}{l}17.72 \\
7747 . \\
4.816\end{array}$ & $\begin{array}{l}18.11 \\
1765 . \\
4.853\end{array}$ & $\begin{array}{l}18.49 \\
1783 \\
4.890\end{array}$ & $\begin{array}{l}18.87 \\
1800 \\
4.925\end{array}$ \\
\hline 10600 & $\begin{array}{l}\nabla \\
H \\
S\end{array}$ & $\begin{array}{l}14.41 \\
1603 . \\
4.499\end{array}$ & $\begin{array}{l}14.90 \\
1626 . \\
4.550\end{array}$ & $\begin{array}{l}15.38 \\
1648 . \\
4.598\end{array}$ & $\begin{array}{l}15.83 \\
1669 . \\
4.644\end{array}$ & $\begin{array}{l}16.27 \\
1689 . \\
4.688\end{array}$ & $\begin{array}{l}16.69 \\
1708 . \\
4.729\end{array}$ & $\begin{array}{l}17.10 \\
1727 . \\
4.769\end{array}$ & $\begin{array}{l}17.50 \\
1745 . \\
4.808\end{array}$ & $\begin{array}{l}17.89 \\
1763 . \\
4.846\end{array}$ & $\begin{array}{l}18.28 \\
1781 \\
4.882\end{array}$ & $\begin{array}{l}18.65 \\
1798 \\
4.917\end{array}$ \\
\hline
\end{tabular}


Table 10 (Continued)

Temperature range: 170 to $220^{\circ} \mathrm{C}$

\begin{tabular}{|c|c|c|c|c|c|c|c|c|c|c|c|c|c|}
\hline P & & $\mathbf{I}$ & 170 & 175 & 180 & 185 & 190 & 195 & 200 & 205 & 210 & 215 & 220 \\
\hline 16700 & $\begin{array}{l}\mathbf{7} \\
\mathbf{q} \\
\mathbf{5}\end{array}$ & & $\begin{array}{l}16.21 \\
1 \in 00 \\
4=489\end{array}$ & $\begin{array}{l}14.70 \\
16.23 . \\
4.540\end{array}$ & $\begin{array}{l}15.17 \\
1645 . \\
4.589\end{array}$ & $\begin{array}{l}15.63 \\
1566 . \\
4.635\end{array}$ & $\begin{array}{l}16.06 \\
1686 . \\
4.679\end{array}$ & $\begin{array}{l}16.48 \\
1706 . \\
4.721\end{array}$ & $\begin{array}{l}16.89 \\
1725 \\
4.761\end{array}$ & $\begin{array}{l}17.29 \\
1743 . \\
4.800\end{array}$ & $\begin{array}{l}17.68 \\
1762 . \\
2.838\end{array}$ & $\begin{array}{l}18.06 \\
1779 . \\
4.875\end{array}$ & $\begin{array}{l}18.44 \\
1797 . \\
4.910\end{array}$ \\
\hline 10800 & $\begin{array}{l}\mathbf{P} \\
H \\
S\end{array}$ & & $\begin{array}{l}14.01 \\
1557 . \\
4.479\end{array}$ & $\begin{array}{l}14.50 \\
16.20 . \\
4.531\end{array}$ & $\begin{array}{l}14.97 \\
1642 . \\
4.580\end{array}$ & $\begin{array}{l}15.42 \\
1664 . \\
4.626\end{array}$ & $\begin{array}{l}15.86 \\
1684 \\
4.670\end{array}$ & $\begin{array}{l}16.28 \\
1704 \\
4.713\end{array}$ & $\begin{array}{l}16.69 \\
1723 \\
4.753\end{array}$ & $\begin{array}{l}17.09 \\
1741 . \\
4.792\end{array}$ & $\begin{array}{l}17.47 \\
1760 . \\
4.830\end{array}$ & $\begin{array}{l}17.85 \\
1777 \\
4.867\end{array}$ & $\begin{array}{l}18.22 \\
1795 . \\
4.903\end{array}$ \\
\hline 10900 & $\begin{array}{l}\mathrm{I} \\
\mathrm{E} \\
\mathrm{S}\end{array}$ & & $\begin{array}{l}13.81 \\
1594 . \\
4.468\end{array}$ & $\begin{array}{l}14.36 \\
16.17 \\
4.52 i\end{array}$ & $\begin{array}{l}14.78 \\
1640 \\
4.571\end{array}$ & $\begin{array}{l}15.23 \\
1661 \\
4.617\end{array}$ & $\begin{array}{l}15.66 \\
1682 . \\
4.662\end{array}$ & $\begin{array}{l}16.08 \\
1701 . \\
4.704\end{array}$ & $\begin{array}{l}16.49 \\
1721 . \\
4.745\end{array}$ & $\begin{array}{l}16.88 \\
1739 \\
4.785\end{array}$ & $\begin{array}{l}17.27 \\
1758 \\
0.823\end{array}$ & $\begin{array}{l}17.65 \\
1776 \\
4.860\end{array}$ & $\begin{array}{l}18.02 \\
1793 . \\
4.895\end{array}$ \\
\hline 11000 & $\begin{array}{l}D \\
H \\
S\end{array}$ & & $\begin{array}{l}13.62 \\
1591 . \\
4.458\end{array}$ & $\begin{array}{l}14.11 \\
16: 5 . \\
4.5 .12\end{array}$ & $\begin{array}{l}14.58 \\
1637 . \\
4.561\end{array}$ & $\begin{array}{l}15.03 \\
1659 . \\
4.609\end{array}$ & $\begin{array}{l}15.46 \\
1679 \\
4.653\end{array}$ & $\begin{array}{l}15.88 \\
1699 \\
4.696\end{array}$ & $\begin{array}{l}16.29 \\
1718 \\
4.737\end{array}$ & $\begin{array}{l}16.68 \\
1737 \\
4.777\end{array}$ & $\begin{array}{l}17.07 \\
1756 . \\
4.815\end{array}$ & $\begin{array}{l}17.44 \\
1774 \\
4.852\end{array}$ & $\begin{array}{l}17.81 \\
1791 . \\
4.888\end{array}$ \\
\hline 11100 & $\begin{array}{l}\mathbf{Y} \\
\mathbf{H} \\
\mathrm{S}\end{array}$ & & $\begin{array}{l}13.43 \\
15.8 . \\
4.448\end{array}$ & $\begin{array}{l}13.92 \\
16: 2 . \\
4.502\end{array}$ & $\begin{array}{l}14.39 \\
1634 \\
4.552\end{array}$ & $\begin{array}{l}14.84 \\
1 \in 56 . \\
4.600\end{array}$ & $\begin{array}{l}15.27 \\
1677 \\
4.645\end{array}$ & $\begin{array}{l}15.69 \\
1697 \\
4.688\end{array}$ & $\begin{array}{l}16.09 \\
1716 . \\
4.725\end{array}$ & $\begin{array}{l}16.49 \\
1735 . \\
4.769\end{array}$ & $\begin{array}{l}16.87 \\
1754 \\
0.807\end{array}$ & $\begin{array}{l}17.25 \\
1772 . \\
4.845\end{array}$ & $\begin{array}{l}17.61 \\
1789 . \\
4.881\end{array}$ \\
\hline 11200 & $\begin{array}{l}V \\
H \\
S\end{array}$ & & $\begin{array}{l}13.24 \\
15 E 5 . \\
4.438\end{array}$ & $\begin{array}{l}13.73 \\
16 C 9 \\
4.492\end{array}$ & $\begin{array}{l}14.20 \\
1632 . \\
4.543\end{array}$ & $\begin{array}{l}14.65 \\
1653 \\
4.591\end{array}$ & $\begin{array}{l}15.08 \\
1674 \\
4.636\end{array}$ & $\begin{array}{l}15.50 \\
1695 \\
4.680\end{array}$ & $\begin{array}{l}15.90 \\
1714 \\
4.721\end{array}$ & $\begin{array}{l}16.30 \\
1733 . \\
4.761\end{array}$ & $\begin{array}{l}16.68 \\
-752 . \\
4.800\end{array}$ & $\begin{array}{l}17.05 \\
1770 \\
4.837\end{array}$ & $\begin{array}{l}17.41 \\
1788 . \\
4.874\end{array}$ \\
\hline 11300 & $\begin{array}{l}V \\
H \\
5\end{array}$ & & $\begin{array}{l}13.0 E \\
15 E 1 \\
4.428\end{array}$ & $\begin{array}{l}13.55 \\
1606 . \\
4.483\end{array}$ & $\begin{array}{l}14.02 \\
1629 . \\
4.534\end{array}$ & $\begin{array}{l}14.47 \\
16511 . \\
4.582\end{array}$ & $\begin{array}{l}14.90 \\
1672 . \\
4.628\end{array}$ & $\begin{array}{l}15.31 \\
1692 . \\
4.672\end{array}$ & $\begin{array}{l}15.72 \\
1712 . \\
4.713\end{array}$ & $\begin{array}{l}16.11 \\
1731 . \\
4.754\end{array}$ & $\begin{array}{l}16.49 \\
1750 . \\
4.792\end{array}$ & $\begin{array}{l}16.8 E \\
1768 . \\
4.830\end{array}$ & $\begin{array}{l}17.22 \\
1786 . \\
4.866\end{array}$ \\
\hline 11400 & $\begin{array}{l}7 \\
3 \\
5\end{array}$ & & $\begin{array}{l}12.88 \\
1578 . \\
4.478\end{array}$ & $\begin{array}{l}13.37 \\
1603 . \\
4.473\end{array}$ & $\begin{array}{l}13.84 \\
1626 \\
4.525\end{array}$ & $\begin{array}{l}14.29 \\
1648 \\
4.573\end{array}$ & $\begin{array}{l}14.72 \\
1670 . \\
4.619\end{array}$ & $\begin{array}{l}15.13 \\
1690 . \\
4.663\end{array}$ & $\begin{array}{l}15.53 \\
1710 \\
4.705\end{array}$ & $\begin{array}{l}15.92 \\
1729 . \\
4.746\end{array}$ & $\begin{array}{l}16.30 \\
1748 \\
.4 .785\end{array}$ & $\begin{array}{l}16.67 \\
1766 . \\
4.823\end{array}$ & $\begin{array}{r}17.03 \\
1784 . \\
.4 .859\end{array}$ \\
\hline $11 \leq 00$ & $\begin{array}{l}7 \\
\text { i } \\
\text { in }\end{array}$ & & $\begin{array}{l}12.70 \\
1575 . \\
4.007\end{array}$ & $\begin{array}{l}13.19 \\
1600 \\
4.463\end{array}$ & $\begin{array}{l}13.66 \\
1623 . \\
4.516\end{array}$ & $\begin{array}{l}14.11 \\
16.96 . \\
4.565\end{array}$ & $\begin{array}{l}14.54 \\
1667 \\
4.611\end{array}$ & $\begin{array}{l}14.95 \\
1688 . \\
4.655\end{array}$ & $\begin{array}{l}15.35 \\
1708 . \\
4.698\end{array}$ & $\begin{array}{l}15.74 \\
1727 \\
4.738\end{array}$ & $\begin{array}{l}16.12 \\
1746 . \\
4.777\end{array}$ & $\begin{array}{l}16.48 \\
1764 . \\
4.815\end{array}$ & $\begin{array}{l}16.84 \\
1782 \\
4.852\end{array}$ \\
\hline 11600 & $\begin{array}{l}y \\
y \\
s\end{array}$ & & $\begin{array}{l}12.52 \\
1572 . \\
4.397\end{array}$ & $\begin{array}{l}13.02 \\
1597 \\
4.454\end{array}$ & $\begin{array}{l}13.49 \\
1621 . \\
4.506\end{array}$ & $\begin{array}{l}13.93 \\
16.33 \\
4.556\end{array}$ & $\begin{array}{l}14.36 \\
1665 . \\
4.603\end{array}$ & $\begin{array}{l}14.77 \\
1685 \\
4.647\end{array}$ & $\begin{array}{l}15.17 \\
1705 \\
4.690\end{array}$ & $\begin{array}{l}15.56 \\
1725 \\
4.73 i\end{array}$ & $\begin{array}{l}15.93 \\
1744 . \\
4.770\end{array}$ & $\begin{array}{l}16.30 \\
1762 . \\
4.808\end{array}$ & $\begin{array}{l}16.66 \\
1780 . \\
4.845\end{array}$ \\
\hline 11700 & $\begin{array}{l}T \\
H \\
S\end{array}$ & & $\begin{array}{l}12.35 \\
15 t \varepsilon \\
4.387\end{array}$ & $\begin{array}{l}12.34 \\
1597 . \\
4.474\end{array}$ & $\begin{array}{l}13.31 \\
1618 \\
4.497\end{array}$ & $\begin{array}{l}13.76 \\
16.00 \\
4.347\end{array}$ & $\begin{array}{l}14.19 \\
1662 . \\
4.594\end{array}$ & $\begin{array}{l}14.60 \\
1683 \\
4.639\end{array}$ & $\begin{array}{l}15.00 \\
1703 \\
4.682\end{array}$ & $\begin{array}{l}15.38 \\
1723 \\
4.723\end{array}$ & $\begin{array}{l}15.76 \\
1742 . \\
4.763\end{array}$ & $\begin{array}{l}16.12 \\
1760 . \\
4.801\end{array}$ & $\begin{array}{l}16.48 \\
1778 . \\
4.838\end{array}$ \\
\hline 11800 & $\begin{array}{l}\mathbf{y} \\
\mathbf{t} \\
\mathbf{S}\end{array}$ & & $\begin{array}{l}12.18 \\
15 \in 5 \\
6.577\end{array}$ & $\begin{array}{l}12.57 \\
1591 . \\
4.434\end{array}$ & $\begin{array}{l}13.14 \\
1615 . \\
4.488\end{array}$ & $\begin{array}{l}13.59 \\
1638 \\
4.538\end{array}$ & $\begin{array}{l}14.02 \\
1660 . \\
4.586\end{array}$ & $\begin{array}{l}14.43 \\
1681 . \\
4.631\end{array}$ & $\begin{array}{l}14.82 \\
1701 \\
4.674\end{array}$ & $\begin{array}{l}15.21 \\
1721 . \\
4.715\end{array}$ & $\begin{array}{l}15.58 \\
1740 . \\
4.755\end{array}$ & $\begin{array}{l}15.95 \\
1758 \\
4.794\end{array}$ & $\begin{array}{l}16.30 \\
1777 \\
4.831\end{array}$ \\
\hline 11900 & $\begin{array}{l}V \\
H \\
\text { S }\end{array}$ & & $\begin{array}{l}12.01 \\
15 E 2 . \\
4 . \equiv 66\end{array}$ & $\begin{array}{l}12.51 \\
1588 . \\
4.425\end{array}$ & $\begin{array}{l}12.98 \\
1612 . \\
4.479\end{array}$ & $\begin{array}{l}13.42 \\
1635 . \\
4.530\end{array}$ & $\begin{array}{l}13.85 \\
1657 . \\
4.578\end{array}$ & $\begin{array}{l}14.26 \\
1678 . \\
4.623\end{array}$ & $\begin{array}{l}14.65 \\
1699 . \\
4.666\end{array}$ & $\begin{array}{l}15.04 \\
1719 . \\
4.708\end{array}$ & $\begin{array}{l}15.41 \\
1738 . \\
4.748\end{array}$ & $\begin{array}{l}15.77 \\
1756 . \\
4.786\end{array}$ & $\begin{array}{l}16.13 \\
1775 . \\
4.824\end{array}$ \\
\hline
\end{tabular}


Table 10 (Continued)

Temperature range: 170 to $220^{\circ} \mathrm{C}$

\begin{tabular}{|c|c|c|c|c|c|c|c|c|c|c|c|c|c|}
\hline P & & $\mathbf{T}$ & 170 & 175 & 180 & 185 & 190 & 195 & 200 & 205 & 210 & 215 & 220 \\
\hline 12000 & $\begin{array}{l}7 \\
1 \\
5\end{array}$ & & $\begin{array}{l}11.84 \\
1558 . \\
4.356\end{array}$ & $\begin{array}{l}12.34 \\
1585 \\
4.415\end{array}$ & $\begin{array}{l}12.81 \\
1609 \\
4.470\end{array}$ & $\begin{array}{l}13.26 \\
1633 \\
4.521\end{array}$ & $\begin{array}{l}13.68 \\
1655 \\
4.569\end{array}$ & $\begin{array}{l}14.09 \\
1676 \\
4.615\end{array}$ & $\begin{array}{l}14.49 \\
1697 \\
4.659\end{array}$ & $\begin{array}{l}14.87 \\
1716 . \\
4.700\end{array}$ & $\begin{array}{l}15.24 \\
1736 \\
4.740\end{array}$ & $\begin{array}{l}15.60 \\
1755 \\
4.779\end{array}$ & $\begin{array}{l}15.96 \\
1773 \\
4.817\end{array}$ \\
\hline 12200 & $\begin{array}{l}y \\
y \\
S\end{array}$ & & $\begin{array}{l}11.52 \\
1551 . \\
4.335\end{array}$ & $\begin{array}{l}12.02 . \\
1578 . \\
4.396\end{array}$ & $\begin{array}{l}12.49 \\
1603 . \\
4.452\end{array}$ & $\begin{array}{l}12.94 \\
1627 . \\
4.504\end{array}$ & $\begin{array}{l}13.36 \\
1650 . \\
4.553\end{array}$ & $\begin{array}{l}13.77 \\
1671 . \\
4.599\end{array}$ & $\begin{array}{l}14.16 \\
1692 . \\
4.643\end{array}$ & $\begin{array}{l}14.54 \\
1712 . \\
4.685\end{array}$ & $\begin{array}{l}14.91 \\
1732 . \\
4.726\end{array}$ & $\begin{array}{l}15.27 \\
1751 . \\
4.765\end{array}$ & $\begin{array}{l}15.62 \\
1769 . \\
4.803\end{array}$ \\
\hline 12400 & $\begin{array}{l}\mathbf{y} \\
\mathbf{y} \\
\mathrm{s}\end{array}$ & & $\begin{array}{l}11.21 \\
1545 . \\
4.315\end{array}$ & $\begin{array}{l}11.71 \\
1572 . \\
4.376\end{array}$ & $\begin{array}{l}12.18 \\
1598 . \\
4.433\end{array}$ & $\begin{array}{l}12.62 \\
1622 . \\
4.486\end{array}$ & $\begin{array}{l}13.05 \\
1645 . \\
4.536\end{array}$ & $\begin{array}{l}13.45 \\
1667 . \\
4.583\end{array}$ & $\begin{array}{l}13.85 \\
1688 . \\
4.628\end{array}$ & $\begin{array}{l}14.22 \\
1708 . \\
4.670\end{array}$ & $\begin{array}{l}14.59 \\
1728 \\
4.711\end{array}$ & $\begin{array}{l}14.95 \\
1747 \\
4.751\end{array}$ & $\begin{array}{l}15.30 \\
1766 . \\
4.789\end{array}$ \\
\hline 12600 & $\begin{array}{l}\mathbf{T} \\
H \\
S\end{array}$ & & $\begin{array}{l}10.90 \\
1537 \\
4.294\end{array}$ & $\begin{array}{l}11.40 \\
1566 \\
4.357\end{array}$ & $\begin{array}{l}11.88 \\
1592 . \\
4.415\end{array}$ & $\begin{array}{l}12.32 \\
1616 . \\
4.469\end{array}$ & $\begin{array}{l}12.74 \\
1640 \\
4.519\end{array}$ & $\begin{array}{l}13.15 \\
1662 . \\
4.567\end{array}$ & $\begin{array}{l}13.54 \\
1683 \\
4.612\end{array}$ & $\begin{array}{l}13.92 \\
1704 . \\
4.656\end{array}$ & $\begin{array}{l}14.28 \\
1724 \\
4.697\end{array}$ & $\begin{array}{l}14.64 \\
1743 \\
4.737\end{array}$ & $\begin{array}{l}14.98 \\
1762 . \\
4.775\end{array}$ \\
\hline 12800 & $\begin{array}{l}\mathbf{D} \\
\mathbf{H} \\
\mathbf{S}\end{array}$ & & $\begin{array}{l}10.60 \\
1530 . \\
4.273\end{array}$ & $\begin{array}{l}11.11 \\
1559 \\
4.337\end{array}$ & $\begin{array}{l}11.58 \\
1586 . \\
4.397\end{array}$ & $\begin{array}{l}12.03 \\
1611 . \\
4.452\end{array}$ & $\begin{array}{l}12.45 \\
1634 . \\
4.503\end{array}$ & $\begin{array}{l}12.85 \\
1657 \\
4.551\end{array}$ & $\begin{array}{l}13.24 \\
1679 . \\
4.597\end{array}$ & $\begin{array}{l}13.62 \\
1699 \\
4.641\end{array}$ & $\begin{array}{l}13.98 \\
1719 \\
4.683\end{array}$ & $\begin{array}{l}14.33 \\
1739 \\
4.723\end{array}$ & $\begin{array}{l}14.68 \\
1758 . \\
4.762\end{array}$ \\
\hline 13000 & $\begin{array}{l}\nabla \\
\text { U } \\
5\end{array}$ & & $\begin{array}{l}10.31 \\
1523 \\
4.251\end{array}$ & $\begin{array}{l}10.82 \\
1553 \\
4.318\end{array}$ & $\begin{array}{l}11.30 \\
1580 . \\
4.378\end{array}$ & $\begin{array}{l}11.74 \\
1605 . \\
4.434\end{array}$ & $\begin{array}{l}12.16 \\
1629 . \\
4.486\end{array}$ & $\begin{array}{l}12.57 \\
1652 . \\
4.535\end{array}$ & $\begin{array}{l}12.95 \\
1674 . \\
4.582\end{array}$ & $\begin{array}{l}13.33 \\
1695 . \\
4.626\end{array}$ & $\begin{array}{l}13.69 \\
1715 . \\
4.668\end{array}$ & $\begin{array}{l}14.04 \\
1735 . \\
4.709\end{array}$ & $\begin{array}{l}14.38 \\
1754 . \\
4.748\end{array}$ \\
\hline $1: 200$ & $\begin{array}{l}\mathbf{D} \\
\mathbf{H} \\
\mathbf{S}\end{array}$ & & $\begin{array}{l}10.03 \\
1516 . \\
4.230\end{array}$ & $\begin{array}{l}10.54 \\
1546 . \\
4.298\end{array}$ & $\begin{array}{l}11.02 \\
1574 . \\
4.360\end{array}$ & $\begin{array}{l}11.46 \\
1600 . \\
4.417\end{array}$ & $\begin{array}{l}11.89 \\
1624 \\
4.470\end{array}$ & $\begin{array}{l}12.29 \\
1647 . \\
4.520\end{array}$ & $\begin{array}{l}12.68 \\
1669 \\
4.567\end{array}$ & $\begin{array}{l}13.05 \\
1691 . \\
4.611\end{array}$ & $\begin{array}{l}13.41 \\
1711 \\
4.654\end{array}$ & $\begin{array}{l}13.75 \\
1731 . \\
4.695\end{array}$ & $\begin{array}{l}14.09 \\
1751 . \\
4.735\end{array}$ \\
\hline 13400 & $\begin{array}{l}\mathbf{I} \\
\mathbf{H} \\
\mathbf{5}\end{array}$ & & $\begin{array}{l}9.751 \\
1508 \\
4.208\end{array}$ & $\begin{array}{l}10.27 \\
1539 \\
4.278\end{array}$ & $\begin{array}{l}10.75 \\
1568 . \\
4.341\end{array}$ & $\begin{array}{l}11.19 . \\
1594 . \\
4.399\end{array}$ & $\begin{array}{l}11.62 \\
1619 \\
4.453\end{array}$ & $\begin{array}{l}12.02 \\
1642 . \\
4.504\end{array}$ & $\begin{array}{l}12.40 \\
1665 . \\
4.552\end{array}$ & $\begin{array}{l}12.77 \\
1686 \\
4.597\end{array}$ & $\begin{array}{l}13.13 \\
1707 . \\
4.640\end{array}$ & $\begin{array}{l}13.48 \\
1727 . \\
4.682\end{array}$ & $\begin{array}{l}13.81 \\
1747 . \\
4.721\end{array}$ \\
\hline $1 \equiv 600$ & $\begin{array}{l}\mathbf{D} \\
\mathbf{H} \\
\mathbf{S}\end{array}$ & & $\begin{array}{l}9.482 \\
1500 \\
4.187\end{array}$ & $\begin{array}{l}10.00 \\
1532 . \\
4.258\end{array}$ & $\begin{array}{l}10.48 \\
1561 \\
4.323\end{array}$ & $\begin{array}{l}10.93 \\
1588 . \\
4.382\end{array}$ & $\begin{array}{l}11.35 \\
1613 \\
4.437\end{array}$ & $\begin{array}{l}11.76 \\
1637 . \\
4.488\end{array}$ & $\begin{array}{l}12.14 \\
1660 . \\
4.536\end{array}$ & $\begin{array}{l}12.51 \\
1682 . \\
4.582\end{array}$ & $\begin{array}{l}12.86 \\
1703 . \\
4.626\end{array}$ & $\begin{array}{l}13.21 \\
1723 . \\
4.668\end{array}$ & $\begin{array}{l}13.54 \\
1743 . \\
4.7 \mathrm{c8}\end{array}$ \\
\hline 13800 & $\begin{array}{l}\nabla \\
\text { t } \\
\text { S }\end{array}$ & & $\begin{array}{l}9.219 \\
1492 . \\
4.165\end{array}$ & $\begin{array}{l}9.745 \\
1525 . \\
4.238\end{array}$ & $\begin{array}{l}10.23 \\
1555 \\
4.304\end{array}$ & $\begin{array}{l}10.68 \\
1582 . \\
4.365\end{array}$ & $\begin{array}{l}11.10 \\
1608 . \\
4.420\end{array}$ & $\begin{array}{l}11.50 \\
1632 \\
4.472\end{array}$ & $\begin{array}{l}11.88 \\
1655 \\
4.521\end{array}$ & $\begin{array}{l}12.25 \\
1677 . \\
4.568\end{array}$ & $\begin{array}{l}12.61 \\
1699 . \\
4.612\end{array}$ & $\begin{array}{l}12.95 \\
1719 \\
4.654\end{array}$ & $\begin{array}{l}13.28 \\
1739 . \\
4.695\end{array}$ \\
\hline 14000 & $\begin{array}{l}\mathbf{y} \\
\mathbf{H} \\
\mathbf{S}\end{array}$ & & $\begin{array}{l}8.962 \\
1484 . \\
4.143\end{array}$ & $\begin{array}{l}9.493 \\
1518 \\
4.218\end{array}$ & $\begin{array}{l}9.978 \\
1549 . \\
4.286\end{array}$ & $\begin{array}{l}10.43 \\
1577 \\
4.347\end{array}$ & $\begin{array}{l}10.85^{\circ} \\
1603 . \\
4.404\end{array}$ & $\begin{array}{l}11.25 \\
1627 \\
4.457\end{array}$ & $\begin{array}{l}11.63 \\
1651 . \\
4.506\end{array}$ & $\begin{array}{l}12.00 \\
1673 \\
4.553\end{array}$ & $\begin{array}{l}12.35 \\
1694 . \\
4.598\end{array}$ & $\begin{array}{l}12 . E S \\
1715 \\
4.641\end{array}$ & $\begin{array}{l}13.03 \\
1735 \\
4.682\end{array}$ \\
\hline 14200 & $\begin{array}{l}P \\
\mathbf{H} \\
\mathbf{S}\end{array}$ & & $\begin{array}{l}8.712 \\
1476 . \\
4.120\end{array}$ & $\begin{array}{l}9.247 \\
1511 . \\
4.198\end{array}$ & $\begin{array}{l}9.734 \\
1542 \\
4.267\end{array}$ & $\begin{array}{l}10.19 \\
1571 . \\
0.330\end{array}$ & $\begin{array}{l}10.61 \\
1597 . \\
4.388\end{array}$ & $\begin{array}{l}11.01 \\
1622 . \\
4.441\end{array}$ & $\begin{array}{l}11.39 \\
1646 . \\
4.492\end{array}$ & $\begin{array}{l}11.76 \\
1668 \\
4.539\end{array}$ & $\begin{array}{l}12.11 \\
1690 . \\
4.584\end{array}$ & $\begin{array}{l}12.45 \\
1711 \\
4.627\end{array}$ & $\begin{array}{l}12.78 \\
1731 . \\
4.669\end{array}$ \\
\hline 14400 & $\begin{array}{l}V \\
H \\
S\end{array}$ & & $\begin{array}{l}8.467 \\
1468 . \\
4.097\end{array}$ & $\begin{array}{l}9.007 \\
1504 . \\
4.178\end{array}$ & $\begin{array}{l}9.497 \\
1535 . \\
4.248\end{array}$ & $\begin{array}{l}9.950 \\
1565 . \\
4.312\end{array}$ & $\begin{array}{l}10.37 \\
1592 . \\
4.371\end{array}$ & $\begin{array}{l}10.77 \\
1617 . \\
4.426\end{array}$ & $\begin{array}{l}11.16 \\
1641 \\
4.477\end{array}$ & $\begin{array}{l}11.52 \\
1664 . \\
4.525\end{array}$ & $\begin{array}{l}11.87 \\
1686 . \\
4.571\end{array}$ & $\begin{array}{l}12.21 \\
1707 \\
4.614\end{array}$ & $\begin{array}{l}12.54 \\
1728 \\
4.656\end{array}$ \\
\hline
\end{tabular}


Table 10 (Continued)

Temperature range: 170 to $220^{\circ} \mathrm{C}$

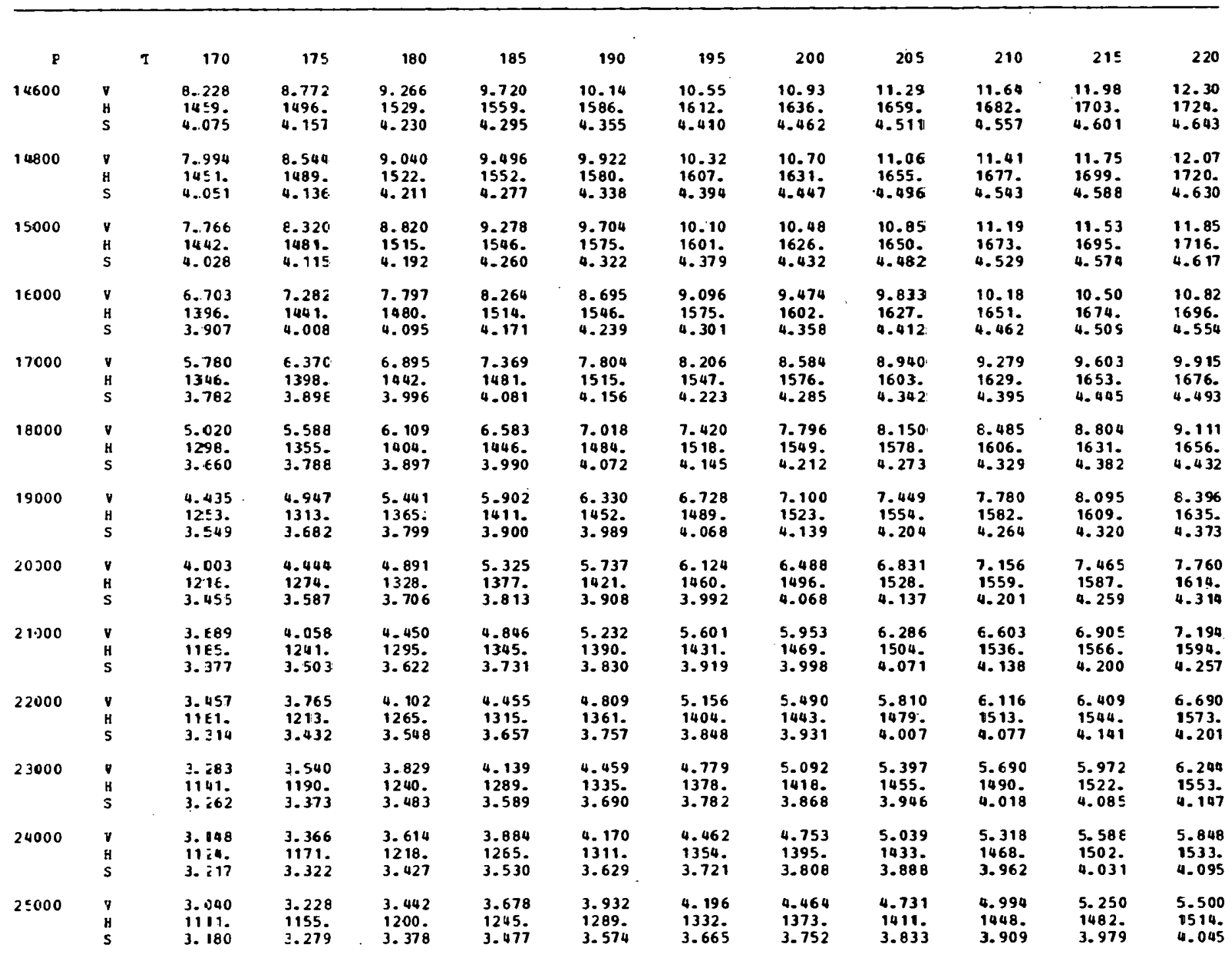


Table 10 (Continued)

Temperature range: 170 to $220^{\circ} \mathrm{C}$

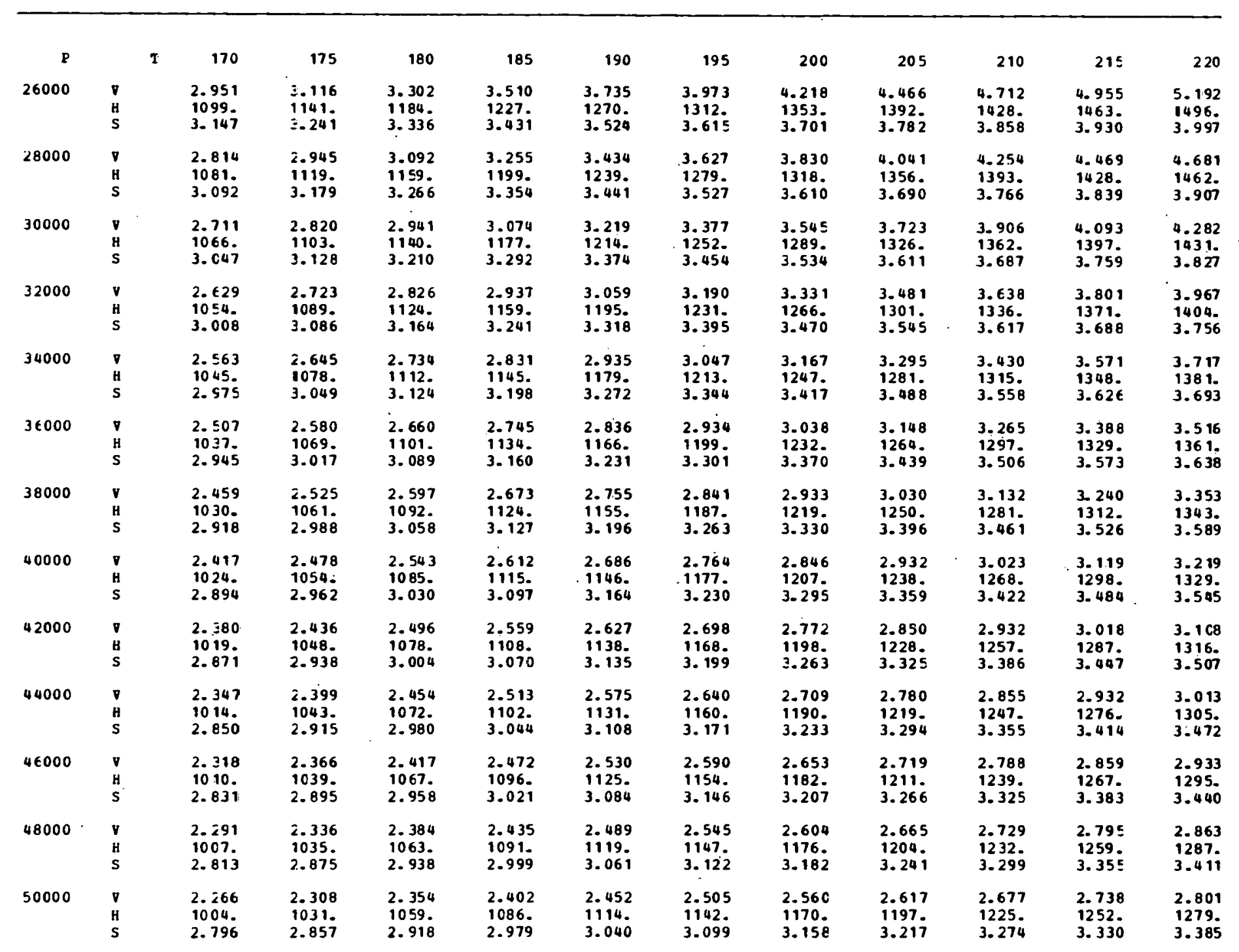


Table 10 (Continued)

Temperature range: 225 to $275^{\circ} \mathrm{C}$

\begin{tabular}{|c|c|c|c|c|c|c|c|c|c|c|c|c|}
\hline P & & 225 & 23:3 & 235 & 240 & 245 & 250 & 255 & 260 & 265 & 270 & 275 \\
\hline 5 & $\begin{array}{l}\mathbf{Y} \\
\mathbf{H} \\
\mathbf{S}\end{array}$ & $\begin{array}{r}48630 \\
1986 \\
8.954\end{array}$ & $\begin{array}{r}49120 . \\
1999 . \\
8.973\end{array}$ & $\begin{array}{r}49610 . \\
2011 . \\
9.003\end{array}$ & $\begin{array}{r}50090 . \\
2024 \\
9.027\end{array}$ & $\begin{array}{r}50580 \\
2036 \\
9.05 i\end{array}$ & $\begin{array}{r}51070 \\
2049 \\
9.075\end{array}$ & $\begin{array}{r}51560 . \\
2061 . \\
9.099\end{array}$ & $\begin{array}{r}52050 \\
2074 . \\
9.123\end{array}$ & $\begin{array}{r}52540 \\
2086 \\
9.147\end{array}$ & $\begin{array}{r}53020 \\
2099 \\
9.170\end{array}$ & $\begin{array}{r}53510 . \\
2112 . \\
9.194\end{array}$ \\
\hline 10 & $\begin{array}{l}\mathrm{V} \\
\mathrm{u} \\
\mathrm{S}\end{array}$ & $\begin{array}{r}24330 \\
1986 \\
8.615\end{array}$ & $\begin{array}{r}24560 \\
1999 . \\
8.640\end{array}$ & $\begin{array}{r}24800 \\
2011= \\
8.664\end{array}$ & $\begin{array}{r}25050 \\
2024 . \\
8.6 \pm 9\end{array}$ & $\begin{array}{r}25290 \\
2036 \\
8.713\end{array}$ & $\begin{array}{r}25530 \\
2049 \\
8.737\end{array}$ & $\begin{array}{r}25780 \\
2061 \\
8.761\end{array}$ & $\begin{array}{r}26020 \\
2074 \\
8.785\end{array}$ & $\begin{array}{c}26270 \\
2086 \\
8.808\end{array}$ & $\begin{array}{r}26510 \\
2099 \\
8.832\end{array}$ & $\begin{array}{r}26750 . \\
2112 . \\
8.855\end{array}$ \\
\hline so & $\begin{array}{l}y \\
y \\
5\end{array}$ & $\begin{array}{l}48 E 0 . \\
19 E \epsilon . \\
7.829\end{array}$ & $\begin{array}{l}4910 . \\
1998 . \\
7.853\end{array}$ & $\begin{array}{l}4960 . \\
2011 . \\
7.878\end{array}$ & $\begin{array}{l}5011 . \\
2023 . \\
7.992\end{array}$ & $\begin{array}{l}5060 . \\
2035 . \\
7.926\end{array}$ & $\begin{array}{l}5100 . \\
2048 . \\
7.950\end{array}$ & $\begin{array}{l}5150 . \\
2061 . \\
7.974\end{array}$ & $\begin{array}{l}5200 . \\
2073 . \\
7.998\end{array}$ & $\begin{array}{l}5250 . \\
2086 \\
8.022\end{array}$ & $\begin{array}{l}5300 . \\
2099 \\
8.045\end{array}$ & $\begin{array}{l}5350 . \\
2111 . \\
8.069\end{array}$ \\
\hline 100 & $\begin{array}{l}V \\
H \\
S\end{array}$ & $\begin{array}{l}2430 . \\
1985 . \\
7.489\end{array}$ & $\begin{array}{l}2450 . \\
1997 . \\
7.51\end{array}$ & $\begin{array}{l}2480 . \\
2010 . \\
7.538\end{array}$ & $\begin{array}{l}2500 . \\
2022 . \\
7.563\end{array}$ & $\begin{array}{l}2530 . \\
2035 . \\
7.587\end{array}$ & $\begin{array}{l}2550 \\
2047 \\
7.611\end{array}$ & $\begin{array}{l}2580 . \\
2060 \\
7.635\end{array}$ & $\begin{array}{l}2600 \\
2073 \\
7.659\end{array}$ & $\begin{array}{l}2620 \\
2085 \\
7.683\end{array}$ & $\begin{array}{l}2650 . \\
2098 . \\
7.70 €\end{array}$ & $\begin{array}{l}2670 . \\
2111 . \\
7.730\end{array}$ \\
\hline 101.33 & $\begin{array}{l}v \\
\mathrm{u} \\
\mathrm{s}\end{array}$ & $\begin{array}{l}2400 . \\
1985 . \\
7.483\end{array}$ & $\begin{array}{l}2420- \\
1997 . \\
7.507\end{array}$ & $\begin{array}{l}2440 . \\
2010 . \\
7.532\end{array}$ & $\begin{array}{l}2470 . \\
2022 . \\
7.556\end{array}$ & $\begin{array}{l}2490 . \\
2035 . \\
7.580\end{array}$ & $\begin{array}{l}2520 . \\
2047 . \\
7.605\end{array}$ & $\begin{array}{l}2540 . \\
2060 . \\
7.629\end{array}$ & $\begin{array}{l}2570 . \\
2073 . \\
7.652\end{array}$ & $\begin{array}{l}2590 . \\
2085 \\
7.676\end{array}$ & $\begin{array}{l}2610 . \\
2098 . \\
7.700\end{array}$ & $\begin{array}{l}2640 . \\
2111 . \\
7.723\end{array}$ \\
\hline 200 & $\begin{array}{l}V \\
H \\
S\end{array}$ & $\begin{array}{l}1210 . \\
1983 . \\
7.148\end{array}$ & $\begin{array}{l}1220- \\
19965 \\
7.17 \mathrm{I}\end{array}$ & $\begin{array}{l}1240 . \\
2008 . \\
7.198\end{array}$ & $\begin{array}{l}1250 . \\
2021 . \\
7.212\end{array}$ & $\begin{array}{l}1260 . \\
2033 . \\
7.247\end{array}$ & $\begin{array}{l}1270 . \\
2046 \\
7.271\end{array}$ & $\begin{array}{l}1290 . \\
2059 . \\
7.295\end{array}$ & $\begin{array}{l}1300 \\
2071: \\
7.319\end{array}$ & $\begin{array}{l}1310 . \\
2084 \\
7.342\end{array}$ & $\begin{array}{l}1320 \\
2097 \\
7.366\end{array}$ & $\begin{array}{l}1340 . \\
2109 . \\
7.389\end{array}$ \\
\hline 300 & $\begin{array}{l}\mathrm{I} \\
\mathrm{H} \\
\mathrm{S}\end{array}$ & $\begin{array}{l}80 \epsilon .9 \\
1982 . \\
6.948\end{array}$ & $\begin{array}{l}\text { E15.5 } \\
1994 . \\
6.973\end{array}$ & $\begin{array}{l}823.4 \\
2007 . \\
6.998\end{array}$ & $\begin{array}{l}831.7 \\
2019.7 \\
7.022\end{array}$ & $\begin{array}{l}839.9 \\
2032 . \\
7.047\end{array}$ & $\begin{array}{l}848.2 \\
2045 . \\
7.071\end{array}$ & $\begin{array}{l}856.4 \\
2057 \\
7.095\end{array}$ & $\begin{array}{l}864.6 \\
2070 . \\
7.119\end{array}$ & $\begin{array}{l}872.9 \\
2083 . \\
7.142\end{array}$ & $\begin{array}{l}881.1 \\
2095 . \\
7.166\end{array}$ & $\begin{array}{l}889.3 \\
2108 . \\
7.190\end{array}$ \\
\hline 400 & $\begin{array}{l}7 \\
H \\
S\end{array}$ & $\begin{array}{l}604.3 \\
1980 . \\
6.805\end{array}$ & $\begin{array}{l}610.5 \\
1993 . \\
6.830\end{array}$ & $\begin{array}{l}616.7 \\
2005 . \\
6.855\end{array}$ & $\begin{array}{l}622.9 \\
2018 . \\
6.880\end{array}$ & $\begin{array}{l}629.2 \\
2031 . \\
6.904\end{array}$ & $\begin{array}{l}635.4 \\
2043 . \\
6.928\end{array}$ & $\begin{array}{l}641.6 \\
2056 . \\
6.952\end{array}$ & $\begin{array}{l}647.8 \\
2069 . \\
6.976\end{array}$ & $\begin{array}{l}654.0 \\
2081 . \\
7.000\end{array}$ & $\begin{array}{l}660.2 \\
2094 . \\
7.024\end{array}$ & $\begin{array}{l}666.4 \\
2107 \\
7.047\end{array}$ \\
\hline 500 & $\begin{array}{l}7 \\
4 \\
S\end{array}$ & $\begin{array}{l}482.7 \\
1979 . \\
5.694\end{array}$ & $\begin{array}{l}487.7 \\
1991 . \\
6.719\end{array}$ & $\begin{array}{l}492.7 \\
2004 . \\
6.764\end{array}$ & $\begin{array}{l}497.7 \\
2017 \\
6.719\end{array}$ & $\begin{array}{l}502.7 \\
2029 . \\
6.793\end{array}$ & $\begin{array}{l}507.7 \\
2042 . \\
6.817\end{array}$ & $\begin{array}{l}512.7 \\
2054 . \\
8.841\end{array}$ & $\begin{array}{l}517.6 \\
2067 . \\
6.865\end{array}$ & $\begin{array}{l}522.6 \\
2080 . \\
6.889\end{array}$ & $\begin{array}{l}527.6 \\
2093 . \\
6.913\end{array}$ & $\begin{array}{l}532.6 \\
2106 . \\
6.937\end{array}$ \\
\hline 600 & $\begin{array}{l}V \\
H \\
S\end{array}$ & $\begin{array}{l}401.7 \\
1977 . \\
6.603\end{array}$ & $\begin{array}{l}405.8 \\
1990 . \\
6.628\end{array}$ & $\begin{array}{l}410.0 \\
2002 . \\
6.653\end{array}$ & $\begin{array}{l}414.2 \\
2015.5 \\
6.6 \% 7\end{array}$ & $\begin{array}{l}18.4 \\
2028 . \\
6.702\end{array}$ & $\begin{array}{l}422.5 \\
2040 . \\
6.726\end{array}$ & $\begin{array}{l}126.7 \\
2053 . \\
6.750\end{array}$ & $\begin{array}{l}430.9 \\
2066 \\
6.774\end{array}$ & $\begin{array}{l}435.0 \\
2079 . \\
6.798\end{array}$ & $\begin{array}{l}439.2 \\
2092 . \\
6.822\end{array}$ & $\begin{array}{l}443.4 \\
2104 . \\
6.846\end{array}$ \\
\hline 705 & $\begin{array}{l}\nabla \\
H \\
S\end{array}$ & $\begin{array}{l}343.8 \\
1976 . \\
5.525\end{array}$ & $\begin{array}{l}347.5 \\
1988 . \\
6.550\end{array}$ & $\begin{array}{l}351.0 \\
2001 . \\
6.575\end{array}$ & $\begin{array}{l}354.6 \\
2012 . \\
6.600\end{array}$ & $\begin{array}{l}358.1 \\
2026 . \\
6.625\end{array}$ & $\begin{array}{l}361.7 \\
2039 \\
6.649\end{array}$ & $\begin{array}{l}165.3 \\
2052 . \\
6.673\end{array}$ & $\begin{array}{l}368.9 \\
2065 \\
6.697\end{array}$ & $\begin{array}{l}372.5 \\
2077 . \\
6.72 i\end{array}$ & $\begin{array}{l}376.1 \\
2090 . \\
6.745\end{array}$ & $\begin{array}{l}379.7 \\
2103 . \\
6.769\end{array}$ \\
\hline $80 \mathrm{~J}$ & $\begin{array}{l}\text { V } \\
\text { H } \\
\text { S }\end{array}$ & $\begin{array}{l}300.3 \\
1974 . \\
5.458\end{array}$ & $\begin{array}{l}303.5 \\
1987 \\
6.485\end{array}$ & $\begin{array}{l}306.7 \\
2006 . \\
6.5 C 8\end{array}$ & $\begin{array}{l}309.8 \\
2012 . \\
6.5 \$ 3\end{array}$ & $\begin{array}{l}313.0 \\
2025 . \\
6.557\end{array}$ & $\begin{array}{l}316.1 \\
2038 . \\
6.582\end{array}$ & $\begin{array}{l}319.3 \\
2050 . \\
E .606\end{array}$ & $\begin{array}{l}322.4 \\
2063 . \\
6.630\end{array}$ & $\begin{array}{l}325.6 \\
2076 \\
6.654\end{array}$ & $\begin{array}{l}328.7 \\
2089 . \\
6.678\end{array}$ & $\begin{array}{l}331.9 \\
2102 . \\
6.702\end{array}$ \\
\hline 901 & $\begin{array}{l}7 \\
H \\
5\end{array}$ & $\begin{array}{l}261.6 \\
1973 . \\
0.398\end{array}$ & $\begin{array}{l}269.5 \\
1985 . \\
6.42 \vdots\end{array}$ & $\begin{array}{l}272.2 \\
1998 . \\
6.448\end{array}$ & $\begin{array}{l}275.0 \\
2019 \\
6.4: 3\end{array}$ & $\begin{array}{l}277.8 \\
2023 . \\
6.498\end{array}$ & $\begin{array}{l}280.7 \\
2036 . \\
6.522\end{array}$ & $\begin{array}{l}283.5 \\
2049 . \\
t .546\end{array}$ & $\begin{array}{l}286.3 \\
2062 . \\
6.571\end{array}$ & $\begin{array}{l}289.1 \\
2075 . \\
6.595\end{array}$ & $\begin{array}{l}291.9 \\
2088 . \\
6.619\end{array}$ & $\begin{array}{l}294.7 \\
2101 . \\
6.642\end{array}$ \\
\hline
\end{tabular}


Table 10 (Continued)

Temperature range: 225 to $275^{\circ} \mathrm{C}$

\begin{tabular}{|c|c|c|c|c|c|c|c|c|c|c|c|c|}
\hline P & I & 225 & 230 & 235 & 240 & 245 & 250 & 255 & 260 & 265 & 270 & 275 \\
\hline 1000 & $\begin{array}{l}7 \\
H \\
S\end{array}$ & $\begin{array}{l}239.5 \\
1971 . \\
6.544\end{array}$ & $\begin{array}{l}242.1 \\
1984 . \\
\epsilon .369\end{array}$ & $\begin{array}{l}244.6 \\
1997 . \\
6.394\end{array}$ & $\begin{array}{l}247.2 \\
2009 . \\
6.419\end{array}$ & $\begin{array}{l}249.7 \\
2022 . \\
6.444\end{array}$ & $\begin{array}{l}252.3 \\
2035 . \\
6.469\end{array}$ & $\begin{array}{l}254.8 \\
2048 . \\
6.493\end{array}$ & $\begin{array}{l}257.4 \\
2061 . \\
6.517\end{array}$ & $\begin{array}{l}259.9 \\
2073 . \\
6.541\end{array}$ & $\begin{array}{l}262.4 \\
2086 \\
6.56 \leq\end{array}$ & $\begin{array}{l}265.0 \\
2099 \\
6.589\end{array}$ \\
\hline 1200 & $\begin{array}{l}7 \\
3 \\
3\end{array}$ & $\begin{array}{l}199.0 \\
19 \notin 8 . \\
6.250\end{array}$ & $\begin{array}{l}201.2 \\
1981 . \\
6.276\end{array}$ & $\begin{array}{l}203.3 \\
1994 . \\
6.301\end{array}$ & $\begin{array}{l}205.4 \\
2006 . \\
6.326\end{array}$ & $\begin{array}{l}207.6 \\
2019 . \\
6.351\end{array}$ & $\begin{array}{l}209.7 \\
2032 . \\
6.376\end{array}$ & $\begin{array}{l}211.9 \\
2045 . \\
6.400\end{array}$ & $\begin{array}{l}214.0 \\
2058 . \\
6.424\end{array}$ & $\begin{array}{l}216.1 \\
2071 . \\
6.449\end{array}$ & $\begin{array}{l}218.3 \\
2084 . \\
6.473\end{array}$ & $\begin{array}{l}220.4 \\
2097 \\
6.496\end{array}$ \\
\hline 1400 & $\begin{array}{l}7 \\
\mathrm{~B} \\
5\end{array}$ & $\begin{array}{l}17 c .0 \\
1965 . \\
6.170\end{array}$ & $\begin{array}{l}171.9 \\
1978 . \\
6.196\end{array}$ & $\begin{array}{l}173.8 \\
1991 . \\
6.221 .\end{array}$ & $\begin{array}{l}175.6 \\
2004 . \\
6.246\end{array}$ & $\begin{array}{l}177.5 \\
2016 . \\
6.271\end{array}$ & $\begin{array}{l}179.3 \\
2029 . \\
6.296\end{array}$ & $\begin{array}{l}181.2 \\
2042 . \\
6.32 i\end{array}$ & $\begin{array}{l}183.0 \\
2055 . \\
6.345\end{array}$ & $\begin{array}{l}184.8 \\
2068 . \\
6.369\end{array}$ & $\begin{array}{l}186.7 \\
2081 . \\
6.394\end{array}$ & $\begin{array}{l}188.5 \\
2094 . \\
6.417\end{array}$ \\
\hline 1600 & $\begin{array}{l}n \\
H \\
5\end{array}$ & $\begin{array}{l}148.3 \\
1962 . \\
6.101\end{array}$ & $\begin{array}{l}150.0 \\
1975 \\
6.126\end{array}$ & $\begin{array}{l}151.6 \\
1988 . \\
6.152\end{array}$ & $\begin{array}{l}153.2 \\
2001 . \\
6.177\end{array}$ & $\begin{array}{l}154.9 \\
2014 . \\
6.202\end{array}$ & $\begin{array}{l}156.5 \\
2027 . \\
6.227\end{array}$ & $\begin{array}{l}158.1 \\
2040 . \\
6.252\end{array}$ & $\begin{array}{l}159.8 \\
2053 . \\
6.276\end{array}$ & $\begin{array}{l}161.4 \\
2066 \\
6.300\end{array}$ & $\begin{array}{l}163.0 \\
2079 . \\
6.325\end{array}$ & $\begin{array}{l}164.6 \\
2092 . \\
6.349\end{array}$ \\
\hline 1800 & $\begin{array}{l}v \\
H \\
S\end{array}$ & $\begin{array}{l}131.4 \\
1959 . \\
6.038\end{array}$ & $\begin{array}{l}132.9 \\
1972 . \\
6.064\end{array}$ & $\begin{array}{l}134.4 \\
1985 \\
6.090\end{array}$ & $\begin{array}{l}135.8 \\
1998 . \\
6.115\end{array}$ & $\begin{array}{l}137.3 \\
2011 . \\
6.140\end{array}$ & $\begin{array}{l}138.8 \\
2024 . \\
6.165\end{array}$ & $\begin{array}{l}140.2 \\
2037 \\
6.190\end{array}$ & $\begin{array}{l}141.7 \\
2050 . \\
6.215\end{array}$ & $\begin{array}{l}143.1 \\
2063 . \\
6.239\end{array}$ & $\begin{array}{l}144.6 \\
2076 \\
6.263\end{array}$ & $\begin{array}{l}146.0 \\
2089 . \\
6.287\end{array}$ \\
\hline 2000 & $\begin{array}{l}\mathbf{v} \\
H \\
S\end{array}$ & $\begin{array}{l}117.9 \\
1956 . \\
5.982\end{array}$ & $\begin{array}{l}119.3 \\
1969 . \\
6.008\end{array}$ & $\begin{array}{l}120.6 \\
1982 . \\
6.034\end{array}$ & $\begin{array}{l}121.9 \\
1995 . \\
6.059\end{array}$ & $\begin{array}{l}123.3 \\
2008 . \\
6.085\end{array}$ & $\begin{array}{l}124.6 \\
2021 . \\
6.110\end{array}$ & $\begin{array}{l}125.9 \\
2034 . \\
6.135\end{array}$ & $\begin{array}{l}127.2 \\
2047 . \\
6.159\end{array}$ & $\begin{array}{l}128.5 \\
2060 . \\
6.184\end{array}$ & $\begin{array}{l}129.9 \\
2073 . \\
6.208\end{array}$ & $\begin{array}{l}131.2 \\
2087 . \\
6.232\end{array}$ \\
\hline 2200 & $\begin{array}{l}D \\
H \\
S\end{array}$ & $\begin{array}{l}106.9 \\
1953 . \\
5.931\end{array}$ & $\begin{array}{l}108.1 \\
1966 . \\
5.957\end{array}$ & $\begin{array}{l}109.3 \\
1979 . \\
5.983\end{array}$ & $\begin{array}{l}110.5 \\
1992 . \\
6.008\end{array}$ & $\begin{array}{l}111.8 \\
2005 \\
6.034\end{array}$ & $\begin{array}{l}113.0 \\
2018 . \\
6.059\end{array}$ & $\begin{array}{l}114.2 \\
2031 . \\
6.084\end{array}$ & $\begin{array}{l}115.4 \\
2044 \\
6.109\end{array}$ & $\begin{array}{l}116.6 \\
2058 . \\
6.133\end{array}$ & $\begin{array}{l}117.8 \\
2071 . \\
6.158\end{array}$ & $\begin{array}{l}119.0 \\
2084 . \\
6.182\end{array}$ \\
\hline 2400 & $\begin{array}{l}\mathbf{V} \\
\mathrm{H} \\
\mathrm{S}\end{array}$ & $\begin{array}{l}97.65 \\
19 \leq 0 \\
5.884\end{array}$ & $\begin{array}{l}98.78 \\
1963 \\
5.940\end{array}$ & $\begin{array}{l}99.91 \\
1976 . \\
5.936\end{array}$ & $\begin{array}{l}101.0 \\
1989 . \\
5.962\end{array}$ & $\begin{array}{l}102.2 \\
2002 . \\
5.987\end{array}$ & $\begin{array}{l}103.3 \\
2015 . \\
6.012\end{array}$ & $\begin{array}{l}104.4 \\
2029 . \\
6.038\end{array}$ & $\begin{array}{l}105.5 \\
2042 . \\
6.062\end{array}$ & $\begin{array}{l}106.6 \\
2055 . \\
6.087\end{array}$ & $\begin{array}{l}107.7 \\
2068 . \\
6.112\end{array}$ & $\begin{array}{l}108.9 \\
2082 . \\
6.136\end{array}$ \\
\hline 2600 & $\begin{array}{l}\mathbf{V} \\
H \\
\mathbf{S}\end{array}$ & $\begin{array}{c}89.85 \\
1947 \\
5.840\end{array}$ & $\begin{array}{l}90.90 \\
1960 \\
5.866\end{array}$ & $\begin{array}{l}91.96 \\
1973 . \\
5.892\end{array}$ & $\begin{array}{l}93.01 \\
1986 . \\
5.918\end{array}$ & $\begin{array}{l}94.05 \\
1999 . \\
5.944\end{array}$ & $\begin{array}{l}95.10 \\
2013 . \\
5.969\end{array}$ & $\begin{array}{l}96.14 \\
2026 . \\
5.994\end{array}$ & $\begin{array}{l}97.18 \\
2039 \\
6.019\end{array}$ & $\begin{array}{l}98.21 \\
2052 . \\
6.044\end{array}$ & $\begin{array}{l}99.25 \\
2066 \\
6.069\end{array}$ & $\begin{array}{l}100.3 \\
2079 . \\
6.093\end{array}$ \\
\hline 2800 & $\begin{array}{l}\nabla \\
H \\
S\end{array}$ & $\begin{array}{l}83.16 \\
1943 . \\
5.799\end{array}$ & $\begin{array}{l}84.15 \\
1957 \\
5.825\end{array}$ & $\begin{array}{l}85.14 \\
1970 \\
5.852\end{array}$ & $\begin{array}{l}86.12 \\
1983 . \\
5.878\end{array}$ & $\begin{array}{l}87.10 \\
1997 . \\
5.903\end{array}$ & $\begin{array}{l}88.08 \\
2010 \\
5.929\end{array}$ & $\begin{array}{l}89.05 \\
2023 \\
5.954\end{array}$ & $\begin{array}{l}90.02 \\
2036 \\
5.979\end{array}$ & $\begin{array}{l}90.99 \\
2050 \\
6.004\end{array}$ & $\begin{array}{l}91.96 \\
2063 . \\
6.029\end{array}$ & $\begin{array}{l}92.92 \\
2077 . \\
6.053\end{array}$ \\
\hline 3000 & $\begin{array}{l}7 \\
H \\
S\end{array}$ & $\begin{array}{l}77.37 \\
1940 . \\
5.760\end{array}$ & $\begin{array}{l}78.30 \\
1954 \\
5.787\end{array}$ & $\begin{array}{l}79.23 \\
1967 . \\
5.813\end{array}$ & $\begin{array}{l}80.15 \\
1980 \\
5.840\end{array}$ & $\begin{array}{l}81.07 \\
1994 . \\
5.865\end{array}$ & $\begin{array}{l}81.99 \\
2007 \\
5.891\end{array}$ & $\begin{array}{l}82.91 \\
2020 . \\
5.916\end{array}$ & $\begin{array}{l}83.82 \\
2034 \\
5.942\end{array}$ & $\begin{array}{l}84.73 \\
2047 \\
5.967\end{array}$ & $\begin{array}{l}85.64 \\
2061 . \\
5.992\end{array}$ & $\begin{array}{l}86.55 \\
2074 . \\
6.016\end{array}$ \\
\hline 3200 & $\begin{array}{l}V \\
H \\
S\end{array}$ & $\begin{array}{l}72.29 \\
1937 \\
5.724\end{array}$ & $\begin{array}{l}73.18 \\
1951 \\
5.751\end{array}$ & $\begin{array}{l}74.05 \\
1964 \\
5.777\end{array}$ & $\begin{array}{l}74.93 \\
1977 \\
5.804\end{array}$ & $\begin{array}{l}75.80 \\
1991 . \\
5.830\end{array}$ & $\begin{array}{l}76.67 \\
2004 \\
5.855\end{array}$ & $\begin{array}{l}77.53 \\
2018 . \\
5.881\end{array}$ & $\begin{array}{l}78.39 \\
2031 \\
5.906\end{array}$ & $\begin{array}{l}79.25 \\
2045 \\
5.931\end{array}$ & $\begin{array}{l}80.11 \\
2058 . \\
5.956\end{array}$ & $\begin{array}{l}80.97 \\
2071 . \\
5.981\end{array}$ \\
\hline 3400 & $\begin{array}{l}V \\
H \\
S\end{array}$ & $\begin{array}{l}67.82 \\
1934 . \\
5.690\end{array}$ & $\begin{array}{l}68.66 \\
1948 . \\
5.717\end{array}$ & $\begin{array}{l}69.49 \\
1961 . \\
5.743\end{array}$ & $\begin{array}{l}70.32 \\
1974 . \\
5.770\end{array}$ & $\begin{array}{l}71.14 \\
1988 . \\
5.796\end{array}$ & $\begin{array}{l}71.97 \\
2001 . \\
5.822\end{array}$ & $\begin{array}{l}72.79 \\
2015 . \\
5.847\end{array}$ & $\begin{array}{l}73.61 \\
2028 . \\
5.873\end{array}$ & $\begin{array}{l}74.42 \\
2042 . \\
5.898\end{array}$ & $\begin{array}{l}75.23 \\
2055 . \\
5.923\end{array}$ & $\begin{array}{l}76.04 \\
2069 . \\
5.948\end{array}$ \\
\hline
\end{tabular}


Table 10 (Continued)

Temperature range: 225 to $275^{\circ} \mathrm{C}$

\begin{tabular}{|c|c|c|c|c|c|c|c|c|c|c|c|c|}
\hline $\mathbf{P}$ & I & 225 & 230 & 235 & 240 & 245 & 250 & 255 & 260 & 265 & 270 & 275 \\
\hline 3600 & $\begin{array}{l}\text { i } \\
\text { y } \\
\text { s }\end{array}$ & $\begin{array}{l}63.84 \\
1931 \\
5.657\end{array}$ & $\begin{array}{l}64.64 \\
1944 . \\
5.6894\end{array}$ & $\begin{array}{l}65.43 \\
1958 . \\
5.711\end{array}$ & $\begin{array}{l}66.22 \\
1972 \\
5.737\end{array}$ & $\begin{array}{l}67.01 \\
1985 . \\
5.764\end{array}$ & $\begin{array}{l}67.79 \\
1999 . \\
5.790\end{array}$ & $\begin{array}{l}68.57 \\
2012 \\
5-815\end{array}$ & $\begin{array}{l}69.35 \\
2026 \\
5.84 i\end{array}$ & $\begin{array}{l}70.12 \\
2039 . \\
5.866\end{array}$ & $\begin{array}{l}70.90 \\
2053 \\
5.891\end{array}$ & $\begin{array}{l}71.67 \\
2066 . \\
5.916\end{array}$ \\
\hline 3800 & $\begin{array}{l}\text { V } \\
\text { H } \\
\text { S }\end{array}$ & $\begin{array}{l}50.28 \\
1998 . \\
5.626\end{array}$ & $\begin{array}{l}61.04 \\
1941 \\
5.653\end{array}$ & $\begin{array}{l}61.80 \\
1955 . \\
5.680\end{array}$ & $\begin{array}{l}62.55 \\
1969 \\
5.707\end{array}$ & $\begin{array}{l}63.30 \\
1982 . \\
5.733\end{array}$ & $\begin{array}{l}64.05 \\
1996 . \\
5.759\end{array}$ & $\begin{array}{l}64.80 \\
2009 . \\
5.785\end{array}$ & $\begin{array}{l}65.54 \\
2023 . \\
5.810\end{array}$ & $\begin{array}{l}66.28 \\
2037 . \\
5.836\end{array}$ & $\begin{array}{l}67.02 \\
2050 \\
5.86 i\end{array}$ & $\begin{array}{l}67.75 \\
2064 . \\
5.888\end{array}$ \\
\hline 4000 & $\begin{array}{l}y \\
y \\
s\end{array}$ & $\begin{array}{l}57.07 \\
1925 . \\
5.596\end{array}$ & $\begin{array}{l}57.80 \\
1938 . \\
5.623\end{array}$ & $\begin{array}{l}58.53 \\
1952 . \\
5.650\end{array}$ & $\begin{array}{l}59.25 \\
1966 \\
5.677\end{array}$ & $\begin{array}{l}59.97 \\
1979 . \\
5.704\end{array}$ & $\begin{array}{l}60.69 \\
1993 . \\
5.730\end{array}$ & 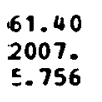 & $\begin{array}{l}62.11 \\
2020 \\
5.782\end{array}$ & $\begin{array}{l}62.82 \\
2034 \\
5.807\end{array}$ & $\begin{array}{l}63.52 \\
2048 . \\
5.832\end{array}$ & $\begin{array}{l}64.23 \\
2061 \\
5.857\end{array}$ \\
\hline 4200 & $\begin{array}{l}\text { D } \\
\text { H } \\
\text { S }\end{array}$ & $\begin{array}{l}54.17 \\
1921 \\
5.567\end{array}$ & $\begin{array}{l}54.87 \\
1935 \\
5.595\end{array}$ & $\begin{array}{l}55.57 \\
1949 . \\
5.622\end{array}$ & $\begin{array}{l}56.26 \\
1963 . \\
5.649\end{array}$ & $\begin{array}{l}56.95 \\
1976 . \\
5.675\end{array}$ & $\begin{array}{l}57.64 \\
1990 \\
5.702\end{array}$ & $\begin{array}{l}58.33 \\
2004 . \\
5.728\end{array}$ & $\begin{array}{l}59.01 \\
2018 . \\
5.754\end{array}$ & $\begin{array}{l}59.69 \\
2031 . \\
5.779\end{array}$ & $\begin{array}{l}60.36 \\
2045 . \\
5.805\end{array}$ & $\begin{array}{l}61.04 \\
2059 . \\
5.830\end{array}$ \\
\hline 4400 & $\begin{array}{l}\text { y } \\
\text { u } \\
\text { s. }\end{array}$ & $\begin{array}{l}51.53 \\
1918 . \\
5.540\end{array}$ & $\begin{array}{l}52.21 \\
1932 \\
5.56 .7\end{array}$ & $\begin{array}{l}52.88 \\
1946 . \\
5.595\end{array}$ & $\begin{array}{l}53.55 \\
1960 \\
5.622\end{array}$ & $\begin{array}{l}54.21 \\
1974 . \\
5.648\end{array}$ & $\begin{array}{l}54.87 \\
1987 \\
5.675\end{array}$ & $\begin{array}{l}55.53 \\
2001 \\
5.701\end{array}$ & $\begin{array}{l}56.19 \\
2015 \\
5.727\end{array}$ & $\begin{array}{l}56.84 \\
20.29 . \\
5.753\end{array}$ & $\begin{array}{l}57.49 \\
2042 . \\
5.778\end{array}$ & $\begin{array}{l}58.14 \\
2056 . \\
5.804\end{array}$ \\
\hline 4600 & $\begin{array}{l}\text { V } \\
\text { H } \\
\text { S }\end{array}$ & $\begin{array}{l}49.13 \\
1995 \\
5.513\end{array}$ & $\begin{array}{l}49.78 \\
1929 . \\
5.54 .1\end{array}$ & $\begin{array}{l}50.42 \\
19.43 . \\
5.568\end{array}$ & $\begin{array}{l}51.37 \\
1957 . \\
5.596\end{array}$ & $\begin{array}{l}51.71 \\
1971 . \\
5.622\end{array}$ & $\begin{array}{l}52.34 \\
1984 . \\
5.649\end{array}$ & $\begin{array}{l}\$ 2.98 \\
1998 \\
\leq .675\end{array}$ & $\begin{array}{l}53.61 \\
2012 . \\
5.701\end{array}$ & $\begin{array}{l}54.24 \\
2026 . \\
5.727\end{array}$ & $\begin{array}{l}54.86 \\
2040 . \\
5.753\end{array}$ & $\begin{array}{l}55.49 \\
2054 \\
5.778\end{array}$ \\
\hline 4800 & $\begin{array}{l}y \\
\text { u } \\
\text { s }\end{array}$ & $\begin{array}{l}46.92 \\
1912 . \\
5.487\end{array}$ & $\begin{array}{l}47.55 \\
1926 \\
5.515\end{array}$ & $\begin{array}{l}48.17 \\
1940 \\
5.543\end{array}$ & $\begin{array}{l}48.79 \\
1954 . \\
5.570\end{array}$ & $\begin{array}{l}49.41 \\
1968 . \\
5.597\end{array}$ & $\begin{array}{l}50.03 \\
1982 . \\
5.624\end{array}$ & $\begin{array}{l}50.64 \\
1996 . \\
5.650\end{array}$ & $\begin{array}{l}51.25 \\
2009 \\
5.677\end{array}$ & $\begin{array}{l}51.85 \\
2023 . \\
5.703\end{array}$ & $\begin{array}{l}52.46 \\
2037 . \\
5.728\end{array}$ & $\begin{array}{l}53.06 \\
2051 \\
5.754\end{array}$ \\
\hline SOCO & $\begin{array}{l}\text { D } \\
\text { H } \\
\text { S }\end{array}$ & $\begin{array}{l}44.86 \\
1909 . \\
5.463\end{array}$ & $\begin{array}{l}45.49 \\
1923 . \\
5.491\end{array}$ & $\begin{array}{l}46.10 \\
1937 . \\
5.518\end{array}$ & $\begin{array}{l}46.70 \\
1951 . \\
5.596\end{array}$ & 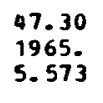 & $\begin{array}{l}47.89 \\
1979 . \\
5.600\end{array}$ & $\begin{array}{l}48.49 \\
1993 . \\
-.626\end{array}$ & $\begin{array}{l}49.07 \\
2007 \\
5.653\end{array}$ & $\begin{array}{l}49.66 \\
2001 \\
5.679\end{array}$ & $\begin{array}{l}50.24 \\
2035 . \\
5.705\end{array}$ & $\begin{array}{l}50.82 \\
2049 . \\
5.730\end{array}$ \\
\hline $52 c 0$ & $\begin{array}{l}\text { D } \\
\text { H } \\
\text { S }\end{array}$ & $\begin{array}{l}43.01 \\
1906 . \\
5.439\end{array}$ & $\begin{array}{l}43.6 \mathrm{C} \\
1920 \mathrm{C} \\
5.467\end{array}$ & $\begin{array}{l}44.19 \\
1934 . \\
5.495\end{array}$ & $\begin{array}{l}94.77 \\
1943 . \\
5.522\end{array}$ & 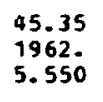 & $\begin{array}{l}45.92 \\
1976 . \\
5.577\end{array}$ & $\begin{array}{l}46.50 \\
1990 . \\
5.603\end{array}$ & $\begin{array}{l}47.07 \\
2004 . \\
5.630\end{array}$ & $\begin{array}{l}47.63 \\
20.18 . \\
5.656\end{array}$ & $\begin{array}{l}48.20 \\
2032 . \\
5.682\end{array}$ & $\begin{array}{l}48.76 \\
2046 \\
5.707\end{array}$ \\
\hline E4c0 & $\begin{array}{l}y \\
y \\
s\end{array}$ & $\begin{array}{l}41.27 \\
1902 . \\
5.415\end{array}$ & $\begin{array}{l}41.84 \\
1917 . \\
5.444\end{array}$ & $\begin{array}{l}42.41 \\
1931 . \\
5.472\end{array}$ & $\begin{array}{l}42.98 \\
1945 . \\
5.439\end{array}$ & $\begin{array}{l}93.54 \\
1959 . \\
5.527\end{array}$ & $\begin{array}{l}44.10 \\
1973 . \\
5.554\end{array}$ & $\begin{array}{l}44.66 \\
7987 \\
5.581\end{array}$ & $\begin{array}{l}45.21 \\
2001 . \\
5.607\end{array}$ & $\begin{array}{l}45.76 \\
20.15 . \\
5.633\end{array}$ & $\begin{array}{l}46.31 \\
2029 . \\
5.659\end{array}$ & $\begin{array}{l}46.85 \\
2043 . \\
5.685\end{array}$ \\
\hline$\leq 600$ & $\begin{array}{l}\text { V } \\
\text { H } \\
S\end{array}$ & $\begin{array}{l}39.6 \epsilon \\
1899 . \\
5.392\end{array}$ & $\begin{array}{l}40.21 \\
19.13 \\
5.421\end{array}$ & $\begin{array}{l}40.77 \\
1928 . \\
5.449\end{array}$ & $\begin{array}{l}41.32 \\
1992 \\
5.477\end{array}$ & $\begin{array}{l}41.86 \\
1956 . \\
5.505\end{array}$ & $\begin{array}{l}42.41 \\
1970 . \\
5.532\end{array}$ & $\begin{array}{l}42.95 \\
1984 . \\
5.559\end{array}$ & $\begin{array}{l}43.48 \\
1999 . \\
5.586\end{array}$ & $\begin{array}{l}40.02 \\
20.33 \\
5.612\end{array}$ & $\begin{array}{l}44.55 \\
2027 . \\
5.638\end{array}$ & $\begin{array}{l}45 . c 8 \\
20.41 \\
5.664\end{array}$ \\
\hline$\leq 800$ & $\begin{array}{l}\text { v } \\
\text { s } \\
\text { s }\end{array}$ & $\begin{array}{l}38.15 \\
1696 \\
5.370\end{array}$ & $\begin{array}{l}38.70 \\
1910 . \\
5.399\end{array}$ & $\begin{array}{l}39.23 \\
1925 . \\
5.428\end{array}$ & $\begin{array}{l}39.77 \\
1939 . \\
5.456\end{array}$ & $\begin{array}{l}40.30 \\
1953 . \\
5.483\end{array}$ & $\begin{array}{l}40.83 \\
1967 . \\
5.511\end{array}$ & $\begin{array}{l}41.35 \\
1982 . \\
5.538\end{array}$ & $\begin{array}{l}41.88 \\
1996 . \\
5.564\end{array}$ & $\begin{array}{l}42.39 \\
20100 \\
5.591\end{array}$ & $\begin{array}{l}42.91 \\
2024 . \\
5.617\end{array}$ & $\begin{array}{l}43.43 \\
2038 . \\
5.643\end{array}$ \\
\hline 6000 & $\begin{array}{l}\mathbf{v} \\
\text { H } \\
\text { S }\end{array}$ & $\begin{array}{l}36.75 \\
1893 \\
5.349\end{array}$ & $\begin{array}{l}37.23 \\
1907 . \\
5.373\end{array}$ & $\begin{array}{l}37.80 \\
1922 . \\
5.40 E\end{array}$ & $\begin{array}{l}38.32 \\
1936 . \\
5.435\end{array}$ & $\begin{array}{l}38.84 \\
1950 . \\
5.462\end{array}$ & $\begin{array}{l}39.36 \\
1965 . \\
5.490\end{array}$ & $\begin{array}{l}39.87 \\
1979 \\
5.517\end{array}$ & $\begin{array}{l}50.38 \\
1993 . \\
5.544\end{array}$ & $\begin{array}{l}40.88 \\
2007 \\
5.570\end{array}$ & $\begin{array}{l}41.38 \\
2022 . \\
5.597\end{array}$ & $\begin{array}{l}41.88 \\
2036 . \\
5.623\end{array}$ \\
\hline
\end{tabular}


Table 10 (Continued)

Temperature range: 225 to $275^{\circ} \mathrm{C}$

\begin{tabular}{|c|c|c|c|c|c|c|c|c|c|c|c|c|c|}
\hline & & & & & & . & & & & & & & \\
\hline $\mathbf{P}$ & & $\mathbf{I}$ & 225 & 230 & 235 & 240 & 245 & 250 & 255 & 260 & 265 & 270 & 275 \\
\hline 6200 & $\begin{array}{l}\mathbf{T} \\
\mathrm{H} \\
\mathbf{5}\end{array}$ & & $\begin{array}{l}35.44 \\
1889 . \\
5.328\end{array}$ & $\begin{array}{l}35.95 \\
1904 \\
5.357\end{array}$ & $\begin{array}{l}36.46 \\
1918 . \\
5.386\end{array}$ & $\begin{array}{l}36.97 \\
1933 . \\
5.414\end{array}$ & $\begin{array}{l}37.48 \\
1947 . \\
5.442\end{array}$ & $\begin{array}{l}37.98 \\
1962 . \\
5.470\end{array}$ & $\begin{array}{l}38.48 \\
1976 \\
5.497\end{array}$ & $\begin{array}{l}38.97 \\
1990 . \\
5.524\end{array}$ & $\begin{array}{l}39.46 \\
2005 \\
5.55 i\end{array}$ & $\begin{array}{l}39.95 \\
2019 \\
5.577\end{array}$ & $\begin{array}{l}40.44 \\
2033 . \\
5.603\end{array}$ \\
\hline 6400 & $\begin{array}{l}\mathbf{y} \\
\mathbf{y} \\
\mathrm{s}\end{array}$ & & $\begin{array}{l}34.20 \\
18 E \epsilon \\
5.307\end{array}$ & $\begin{array}{l}34.71 \\
19011 \\
5.337\end{array}$ & $\begin{array}{l}35.21 \\
1915 . \\
5.365\end{array}$ & $\begin{array}{l}35.71 \\
1930 . \\
5.390\end{array}$ & $\begin{array}{l}36.20 \\
1944 . \\
5.422\end{array}$ & $\begin{array}{l}36.69 \\
1959 . \\
5.450\end{array}$ & $\begin{array}{l}37.17 \\
1973 \\
5.477\end{array}$ & $\begin{array}{l}37.66 \\
1988 . \\
5.504\end{array}$ & $\begin{array}{l}38.14 \\
2002 \\
5.531\end{array}$ & $\begin{array}{l}38.61 \\
2016 . \\
5.558\end{array}$ & $\begin{array}{l}39.09 \\
2031 . \\
5.584\end{array}$ \\
\hline 6600 & $\begin{array}{l}\mathbf{y} \\
\mathbf{H} \\
\mathbf{S}\end{array}$ & & $\begin{array}{l}33.05 \\
1883 . \\
5.287\end{array}$ & $\begin{array}{l}33.54 \\
1898 . \\
5.317\end{array}$ & $\begin{array}{l}34.03 \\
1912 . \\
5.346\end{array}$ & $\begin{array}{l}34.51 \\
1927 . \\
5.374\end{array}$ & $\begin{array}{l}35.00 \\
1942 . \\
5.403\end{array}$ & $\begin{array}{l}35.47 \\
1956 \\
5.431\end{array}$ & $\begin{array}{l}35.95 \\
1971 . \\
5-458\end{array}$ & $\begin{array}{l}36.42 \\
1985 . \\
5.485\end{array}$ & $\begin{array}{l}36.89 \\
1999 . \\
5.512\end{array}$ & $\begin{array}{l}37.35 \\
2014 . \\
5.539\end{array}$ & $\begin{array}{l}37.82 \\
2028 . \\
5.565\end{array}$ \\
\hline 6800 & $\begin{array}{l}\mathbf{v} \\
\mathbf{u} \\
\mathbf{S}\end{array}$ & & $\begin{array}{l}31.96 \\
1880 . \\
5.268\end{array}$ & $\begin{array}{l}32.44 \\
189.4 \\
5.297\end{array}$ & $\begin{array}{l}32.92 \\
1909 \\
5.327\end{array}$ & $\begin{array}{l}33.39 \\
1924 . \\
5.355\end{array}$ & $\begin{array}{l}33.86 \\
1939 . \\
5.384\end{array}$ & $\begin{array}{l}34.33 \\
1953 . \\
5.412\end{array}$ & $\begin{array}{l}34.79 \\
1968 . \\
5.439\end{array}$ & $\begin{array}{l}35.25 \\
1982 \\
5.467\end{array}$ & $\begin{array}{l}35.71 \\
1997 . \\
5.494\end{array}$ & $\begin{array}{l}36.17 \\
2011 \\
5.521\end{array}$ & $\begin{array}{l}36.62 \\
2026 . \\
5.547\end{array}$ \\
\hline 7000 & $\begin{array}{l}\mathbf{V} \\
\mathbf{H} \\
\mathbf{S}\end{array}$ & & $\begin{array}{l}30.93 \\
1876 . \\
5.248\end{array}$ & $\begin{array}{l}31.40 \\
1891 . \\
5.278\end{array}$ & $\begin{array}{l}31.87 \\
1906 . \\
5.308\end{array}$ & $\begin{array}{l}32.33 \\
1921 . \\
5.337\end{array}$ & $\begin{array}{l}32.80 \\
1936 . \\
5.365\end{array}$ & $\begin{array}{l}33.25 \\
1950: \\
5.393\end{array}$ & $\begin{array}{l}33.71 \\
1965 . \\
5.421\end{array}$ & $\begin{array}{l}34.16 \\
1979 . \\
5.449\end{array}$ & $\begin{array}{l}34.60 \\
1994 . \\
5.476\end{array}$ & $\begin{array}{l}35.0 .5 \\
2009 . \\
5.503\end{array}$ & $\begin{array}{l}35.49 \\
2023 . \\
5.529\end{array}$ \\
\hline 7200 & $\begin{array}{l}\mathbf{v} \\
\mathbf{y} \\
\mathbf{S}\end{array}$ & & $\begin{array}{l}29.96 \\
1873 . \\
5.830\end{array}$ & $\begin{array}{l}30.42 \\
1888 . \\
5.260\end{array}$ & $\begin{array}{l}30.88 \\
1903: \\
5.289\end{array}$ & $\begin{array}{l}31.34 \\
1918 . \\
5.318\end{array}$ & $\begin{array}{l}3.1 .79 \\
1933 . \\
5.347\end{array}$ & $\begin{array}{l}32.23 \\
1947 \\
5.375\end{array}$ & $\begin{array}{l}32.68 \\
1962 . \\
5.403\end{array}$ & $\begin{array}{l}33.12 \\
1977 . \\
5.431\end{array}$ & $\begin{array}{l}33.56 \\
1991 . \\
5.458\end{array}$ & $\begin{array}{l}33.99 \\
2006 . \\
5.485\end{array}$ & $\begin{array}{l}34.42 \\
2020 . \\
5.512\end{array}$ \\
\hline 7000 & $\begin{array}{l}\mathbf{V} \\
\mathrm{H} \\
\mathrm{S}\end{array}$ & & $\begin{array}{l}29.04 \\
1870 . \\
5.211\end{array}$ & $\begin{array}{l}29.49 \\
1885 \\
5.24 i\end{array}$ & $\begin{array}{l}29.94 \\
1900 \\
5.271\end{array}$ & $\begin{array}{l}30.39 \\
1915 . \\
5.300\end{array}$ & $\begin{array}{l}30.83 \\
1930 . \\
5.329\end{array}$ & $\begin{array}{l}31.27 \\
1945 . \\
5.358\end{array}$ & $\begin{array}{l}31.71 \\
1959 . \\
5.386\end{array}$ & $\begin{array}{l}32.14 \\
1974 . \\
5.413\end{array}$ & $\begin{array}{l}32.57 \\
1989 . \\
5.441\end{array}$ & $\begin{array}{l}32.99 \\
2003 . \\
5.468\end{array}$ & $\begin{array}{l}33.41 \\
2018 . \\
5.495\end{array}$ \\
\hline 7600 & $\begin{array}{l}\mathbf{y} \\
\mathbf{H} \\
\mathbf{s}\end{array}$ & & $\begin{array}{l}28.11 \\
1866 . \\
5.193\end{array}$ & $\begin{array}{l}28.61 \\
1882 . \\
5.223\end{array}$ & $\begin{array}{l}29.06 \\
1897 . \\
5.253\end{array}$ & $\begin{array}{l}29.49 \\
1912 . \\
5.283\end{array}$ & $\begin{array}{l}29.93 \\
1927 . \\
5.312\end{array}$ & $\begin{array}{l}30.36 \\
1942 . \\
5.340\end{array}$ & $\begin{array}{l}30.78 \\
1956 . \\
5.369\end{array}$ & $\begin{array}{l}31.21 \\
1971 . \\
5.396\end{array}$ & $\begin{array}{l}31.63 \\
1986 . \\
5.424\end{array}$ & $\begin{array}{l}32.04 \\
2001 \\
5.451\end{array}$ & $\begin{array}{l}32.46 \\
2015 . \\
5.478\end{array}$ \\
\hline 7800 & $\begin{array}{l}7 \\
8 \\
\text { S }\end{array}$ & & $\begin{array}{l}27.34 \\
1863 \\
5.175\end{array}$ & $\begin{array}{l}27.78 \\
1878 . \\
5.206\end{array}$ & $\begin{array}{l}28.21 \\
1894 . \\
5.236\end{array}$ & $\begin{array}{l}28.64 \\
1909 . \\
5.266\end{array}$ & $\begin{array}{l}29.07 \\
1924 . \\
5.295\end{array}$ & $\begin{array}{l}29.49 \\
1939 . \\
5.323\end{array}$ & $\begin{array}{l}29.91 \\
1954 . \\
5.352\end{array}$ & $\begin{array}{l}30.32 \\
1968 . \\
5.380\end{array}$ & $\begin{array}{l}30.74 \\
1983 . \\
5.407\end{array}$ & $\begin{array}{l}31.15 \\
1998 . \\
5.435\end{array}$ & $\begin{array}{l}31.55 \\
2013 . \\
5.462\end{array}$ \\
\hline 8000 & $\begin{array}{l}\mathbf{v} \\
\mathbf{H} \\
\mathbf{S}\end{array}$ & & $\begin{array}{l}26.56 \\
1860 . \\
5.158\end{array}$ & $\begin{array}{l}26.99 \\
1875 \\
5.189\end{array}$ & $\begin{array}{l}27.41 \\
1890 . \\
5.219\end{array}$ & $\begin{array}{l}27.84 \\
1906 . \\
5.249\end{array}$ & $\begin{array}{l}28.25 \\
1921 . \\
5.27 .8\end{array}$ & $\begin{array}{l}28.67 \\
1936 \\
5.307\end{array}$ & $\begin{array}{l}29.08 \\
1951 . \\
5.335\end{array}$ & $\begin{array}{l}29.49 \\
1966 . \\
5.363\end{array}$ & $\begin{array}{l}29.89 \\
1981 \\
5.391\end{array}$ & $\begin{array}{l}30.29 \\
1995 . \\
5.419\end{array}$ & $\begin{array}{l}30.69 \\
2010 . \\
5.446\end{array}$ \\
\hline 8200 & $\begin{array}{l}y \\
t \\
s\end{array}$ & & $\begin{array}{l}25.81 \\
1856 . \\
5.141\end{array}$ & $\begin{array}{l}26.23 \\
1872 . \\
5.172\end{array}$ & $\begin{array}{l}26.65 \\
1887 . \\
5.202\end{array}$ & $\begin{array}{l}27.07 \\
1903 . \\
5.232\end{array}$ & $\begin{array}{l}27.48 \\
1918 . \\
5.261\end{array}$ & $\begin{array}{l}27.88 \\
1933 . \\
5.290\end{array}$ & $\begin{array}{l}28.29 \\
1948 . \\
5.319\end{array}$ & $\begin{array}{l}28.69 \\
1963 . \\
5.347\end{array}$ & $\begin{array}{l}29.08 \\
1978 . \\
5.375\end{array}$ & $\begin{array}{l}29.48 \\
1993 . \\
5.403\end{array}$ & $\begin{array}{l}29.87 \\
2008 . \\
5.430\end{array}$ \\
\hline 8400 & $\begin{array}{l}\text { V } \\
\text { u } \\
\text { s }\end{array}$ & & $\begin{array}{l}25.10 \\
18 \leq 3 . \\
5.124\end{array}$ & $\begin{array}{l}25.51 \\
1869 \\
5.155\end{array}$ & $\begin{array}{l}25.93 \\
1884 . \\
5.185\end{array}$ & $\begin{array}{l}26.33 \\
1900 . \\
5.216\end{array}$ & $\begin{array}{l}26.74 \\
1915 . \\
5.245\end{array}$ & $\begin{array}{l}27.14 \\
1930 . \\
5.274\end{array}$ & $\begin{array}{l}27.53 \\
1945 . \\
5.303\end{array}$ & $\begin{array}{l}27.93 \\
1960 . \\
5.332\end{array}$ & $\begin{array}{l}28.32 \\
1975 . \\
5.359\end{array}$ & $\begin{array}{l}28.70 \\
1990 \\
5.387\end{array}$ & $\begin{array}{l}29.09 \\
2005 . \\
5.414\end{array}$ \\
\hline 8600 & $\begin{array}{l}7 \\
4 \\
S\end{array}$ & & $\begin{array}{l}24.42 \\
18 \leq 0 \\
5.107\end{array}$ & $\begin{array}{l}24.83 \\
1865 \\
5.138\end{array}$ & $\begin{array}{l}25.23 \\
1881 . \\
5.169\end{array}$ & $\begin{array}{l}25.64 \\
1897 \\
5.199\end{array}$ & $\begin{array}{l}26.03 \\
1912 . \\
5.229\end{array}$ & $\begin{array}{l}26.43 \\
1927 . \\
5.259\end{array}$ & $\begin{array}{l}26.82 \\
1942 \\
5.287\end{array}$ & $\begin{array}{l}27.20 \\
1957 . \\
5.316\end{array}$ & $\begin{array}{l}27.59 \\
1973 . \\
5.344\end{array}$ & $\begin{array}{l}27.97 \\
1988 \\
5.372\end{array}$ & $\begin{array}{l}28.34 \\
2002 \\
5.399\end{array}$ \\
\hline
\end{tabular}


Table 10 (Continued)

Temperature range: 225 to $275^{\circ} \mathrm{C}$

\begin{tabular}{|c|c|c|c|c|c|c|c|c|c|c|c|c|}
\hline $\mathbf{P}$ & $I$ & 225 & 230 & 235 & 240 & 245 & 250 & 255 & 260 & 265 & 270 & 275 \\
\hline 8800 & $\begin{array}{l}\text { D } \\
\text { H } \\
\text { S }\end{array}$ & $\begin{array}{l}23.77 \\
1806 . \\
5.090\end{array}$ & $\begin{array}{l}24.17 \\
1862 . \\
5.122\end{array}$ & $\begin{array}{l}24.57 \\
1878 . \\
5.153\end{array}$ & $\begin{array}{l}24.97 \\
1893 . \\
5.184\end{array}$ & $\begin{array}{l}25.36 \\
1909 . \\
5.214\end{array}$ & $\begin{array}{l}25.75 \\
1924 . \\
5.243\end{array}$ & $\begin{array}{l}26.13 \\
1940 . \\
5.272\end{array}$ & $\begin{array}{l}26.51 \\
1955 . \\
5.301\end{array}$ & $\begin{array}{l}26.89 \\
1970 . \\
5.329\end{array}$ & $\begin{array}{l}27.26 \\
1985 \\
5.357\end{array}$ & $\begin{array}{l}27.63 \\
2000 . \\
5.384\end{array}$ \\
\hline 9000 & $\begin{array}{l}\mathbf{y} \\
\mathbf{H}\end{array}$ & $\begin{array}{l}23.15 \\
1843 . \\
5.074\end{array}$ & $\begin{array}{l}23.55 \\
1859 . \\
5.106\end{array}$ & $\begin{array}{l}23.94 \\
1.875 \\
5.137\end{array}$ & $\begin{array}{l}24.33 \\
1890 . \\
5.168\end{array}$ & $\begin{array}{l}24.72 \\
1906 . \\
5.198\end{array}$ & $\begin{array}{l}25.10 \\
1921 . \\
5.228\end{array}$ & $\begin{array}{l}25.47 \\
1937 \\
5.257\end{array}$ & $\begin{array}{l}25.85 \\
1952 . \\
5.286\end{array}$ & $\begin{array}{l}26.22 \\
1967 . \\
5.314\end{array}$ & $\begin{array}{l}26.59 \\
1982 . \\
5.342\end{array}$ & $\begin{array}{l}26.95 \\
1997 . \\
5.370\end{array}$ \\
\hline 9200 & $\begin{array}{l}8 \\
8 \\
5\end{array}$ & $\begin{array}{l}22.55 \\
1840 . \\
5.058\end{array}$ & $\begin{array}{l}22.95 \\
1856 . \\
5.090\end{array}$ & $\begin{array}{l}23.34 \\
1372 . \\
5.122\end{array}$ & $\begin{array}{l}23.72 \\
1887 \\
5.153\end{array}$ & $\begin{array}{l}24.10 \\
1903 . \\
5.183\end{array}$ & $\begin{array}{l}24.48 \\
1918 . \\
5.213\end{array}$ & $\begin{array}{l}24.85 \\
1934 . \\
5.242\end{array}$ & $\begin{array}{l}25.22 \\
1949 \\
5.271\end{array}$ & $\begin{array}{l}25.58 \\
1964 . \\
5.299\end{array}$ & $\begin{array}{l}25.94 \\
1980 \\
5.327\end{array}$ & $\begin{array}{l}26.30 \\
1995 . \\
5.355\end{array}$ \\
\hline 9400 & $\begin{array}{l}1 \\
\text { H } \\
\text { S }\end{array}$ & $\begin{array}{l}21.99 \\
1836 . \\
5.043\end{array}$ & $\begin{array}{l}22.37 \\
1852 . \\
5.075\end{array}$ & $\begin{array}{l}22.76 \\
1868 . \\
5.106\end{array}$ & $\begin{array}{l}23.14 \\
1884 . \\
5.137\end{array}$ & $\begin{array}{l}23.51 \\
1900 . \\
5.168\end{array}$ & $\begin{array}{l}23.88 \\
1915 . \\
5.198\end{array}$ & $\begin{array}{l}24.25 \\
1931 \\
5.227\end{array}$ & $\begin{array}{l}24.61 \\
1946 . \\
5.256\end{array}$ & $\begin{array}{l}24.97 \\
1962 . \\
5.285\end{array}$ & $\begin{array}{l}25.33 \\
1977 \\
5.313\end{array}$ & $\begin{array}{l}25.68 \\
1992 . \\
5.341\end{array}$ \\
\hline 9600 & $\begin{array}{l}0 \\
\mathbf{H} \\
\mathbf{5}\end{array}$ & $\begin{array}{l}21.44 \\
1833 \\
5.027\end{array}$ & $\begin{array}{l}21.82 \\
1849 . \\
5.059\end{array}$ & $\begin{array}{l}22.20 \\
1865 \\
5.091\end{array}$ & $\begin{array}{l}22.58 \\
1881 . \\
5.122\end{array}$ & $\begin{array}{l}22.94 \\
1897 \\
5.153\end{array}$ & $\begin{array}{l}23.31 \\
1913 . \\
5.183\end{array}$ & $\begin{array}{l}23.67 \\
1928 . \\
5.213\end{array}$ & $\begin{array}{l}24.03 \\
1944 . \\
5.242\end{array}$ & $\begin{array}{l}24.38 \\
1959 . \\
5.271\end{array}$ & $\begin{array}{l}24.73 \\
1974 . \\
5.299\end{array}$ & $\begin{array}{l}25.08 \\
1990 . \\
5.327\end{array}$ \\
\hline 9800 & $\begin{array}{l}\mathbf{Y} \\
\mathrm{H} \\
\mathrm{S}\end{array}$ & $\begin{array}{l}20.92 \\
18.99 \\
5.012\end{array}$ & $\begin{array}{l}21.30 \\
1846 \\
5.344\end{array}$ & $\begin{array}{l}21.67 \\
1862 . \\
5.076\end{array}$ & $\begin{array}{l}22.04 \\
1878 \\
5.108\end{array}$ & $\begin{array}{l}22.40 \\
1894 . \\
5.139\end{array}$ & $\begin{array}{l}22.76 \\
1910 . \\
5.169\end{array}$ & $\begin{array}{l}23.12 \\
1925 \\
5.199\end{array}$ & $\begin{array}{l}23.47 \\
1941 . \\
5.228\end{array}$ & $\begin{array}{l}23.82 \\
1956 . \\
5.257\end{array}$ & $\begin{array}{l}24.17 \\
1972 . \\
5.285\end{array}$ & $\begin{array}{l}24.51 \\
1987 \\
5.313\end{array}$ \\
\hline 10000 & $\begin{array}{l}\mathbf{V} \\
\mathbf{H} \\
\mathbf{S}\end{array}$ & $\begin{array}{l}20.41 \\
1826 \\
4.996\end{array}$ & $\begin{array}{l}20.79 \\
1802 \\
5.329\end{array}$ & $\begin{array}{l}21.16 \\
18 \leq 9 . \\
5.062\end{array}$ & $\begin{array}{l}21.52 \\
1875 . \\
5.093\end{array}$ & $\begin{array}{l}21.88 \\
1891 . \\
5.124\end{array}$ & $\begin{array}{l}22.24 \\
1907 \\
5.155\end{array}$ & $\begin{array}{l}22.55 \\
1922 . \\
5-185\end{array}$ & $\begin{array}{l}22.94 \\
1938 . \\
5.214\end{array}$ & $\begin{array}{l}23.28 \\
1954 . \\
5.243\end{array}$ & $\begin{array}{l}23.62 \\
1969 \\
5.272\end{array}$ & $\begin{array}{l}23.96 \\
1984 . \\
5.300\end{array}$ \\
\hline $1<100$ & $\begin{array}{l}\mathbf{D} \\
H \\
\mathbf{S}\end{array}$ & $\begin{array}{l}20.17 \\
1824 . \\
4.989\end{array}$ & $\begin{array}{l}20.54 \\
1841 \\
5.122\end{array}$ & $\begin{array}{l}20.91 \\
1957 \\
5.054\end{array}$ & $\begin{array}{l}21.27 \\
1873 . \\
5.086\end{array}$ & $\begin{array}{l}21.63 \\
1889 \\
5.117\end{array}$ & $\begin{array}{l}21.98 \\
1905 \\
5.148\end{array}$ & $\begin{array}{l}22.33 \\
1921 . \\
5.178\end{array}$ & $\begin{array}{l}22.88 \\
1937 \\
5.207\end{array}$ & $\begin{array}{l}23.02 \\
19 \leq 2 . \\
5.236\end{array}$ & $\begin{array}{l}23.36 \\
1968 . \\
5.265\end{array}$ & $\begin{array}{l}23.69 \\
1983 . \\
5.293\end{array}$ \\
\hline $1 C 200$ & $\begin{array}{l}\mathbf{y} \\
\mathrm{u} \\
\mathrm{s}\end{array}$ & $\begin{array}{l}19.93 \\
1822 . \\
4.981\end{array}$ & $\begin{array}{l}20.30 \\
1839 \\
5.1015\end{array}$ & $\begin{array}{l}20.67 \\
1855 \\
5.047\end{array}$ & $\begin{array}{l}21.03 \\
1872 \\
5.079\end{array}$ & $\begin{array}{l}21.38 \\
1888 \\
5.110\end{array}$ & $\begin{array}{l}21.73 \\
1904 \\
5.14 i\end{array}$ & $\begin{array}{l}22.08 \\
1920 \\
5.171\end{array}$ & $\begin{array}{l}22.02 \\
1935 \\
5.200\end{array}$ & $\begin{array}{l}22.76 \\
1951 . \\
5.229\end{array}$ & $\begin{array}{l}23.10 \\
1966 . \\
5.25 E\end{array}$ & $\begin{array}{l}23.43 \\
1982 . \\
5.286\end{array}$ \\
\hline 16300 & $\begin{array}{l}V \\
H \\
S\end{array}$ & $\begin{array}{l}19.70 \\
1821 . \\
4.974\end{array}$ & $\begin{array}{l}20.06 \\
1837 \\
5.007\end{array}$ & $\begin{array}{l}20.43 \\
1854 . \\
5.040\end{array}$ & $\begin{array}{l}20.78 \\
1870 . \\
5.072\end{array}$ & $\begin{array}{l}21.14 \\
1886 . \\
5.103\end{array}$ & $\begin{array}{l}21.49 \\
1902 . \\
5.134\end{array}$ & $\begin{array}{l}21.83 \\
1518 . \\
5.164\end{array}$ & $\begin{array}{l}22.17 \\
1934 . \\
5.193\end{array}$ & $\begin{array}{l}22.51 \\
1949 . \\
5.223\end{array}$ & $\begin{array}{l}22.84 \\
1965 \\
5.251\end{array}$ & $\begin{array}{l}23.17 \\
1980 \\
5.280\end{array}$ \\
\hline 10400 & $\begin{array}{l}\mathbf{D} \\
\mathrm{H} \\
\mathrm{S}\end{array}$ & $\begin{array}{l}19.47 \\
1819 . \\
4.967\end{array}$ & $\begin{array}{l}19.83 \\
1836 \\
5.000\end{array}$ & $\begin{array}{l}20.19 \\
1852 \\
5.033\end{array}$ & $\begin{array}{l}20.55 \\
1869 \\
5.065\end{array}$ & $\begin{array}{l}20.90 \\
1885 \\
5.096\end{array}$ & $\begin{array}{l}21.25 \\
19011 \\
5.127\end{array}$ & $\begin{array}{l}21.59 \\
1917 \\
5.157\end{array}$ & $\begin{array}{l}21.93 \\
1932 \\
5.187\end{array}$ & $\begin{array}{l}22.26 \\
1948 . \\
5.216\end{array}$ & $\begin{array}{l}22.59 \\
1964 . \\
5.245\end{array}$ & $\begin{array}{l}22.92 \\
1979 . \\
5.273\end{array}$ \\
\hline $10 \leq 00$ & $\begin{array}{l}\mathbf{D} \\
\mathrm{H} \\
\mathrm{S}\end{array}$ & $\begin{array}{l}19.24 \\
1817 \\
4.959\end{array}$ & $\begin{array}{l}19.60 \\
1814 \\
4.993\end{array}$ & $\begin{array}{l}19.96 \\
1851 \\
5.025\end{array}$ & $\begin{array}{l}20.32 \\
1867 . \\
5.058\end{array}$ & $\begin{array}{l}20.66 \\
1883 \\
5.089\end{array}$ & $\begin{array}{l}21.01 \\
1899 \\
5.120\end{array}$ & $\begin{array}{l}21.35 \\
1915 \\
5.150\end{array}$ & $\begin{array}{l}21.69 \\
1931 . \\
5.180\end{array}$ & $\begin{array}{l}22.02 \\
1947 . \\
5.209\end{array}$ & $\begin{array}{l}22.35 \\
1962 . \\
5.238\end{array}$ & $\begin{array}{l}22.67 \\
1978 . \\
5.267\end{array}$ \\
\hline 10600 & $\begin{array}{l}7 \\
H \\
S\end{array}$ & $\begin{array}{l}19.02 \\
1816 . \\
4.952\end{array}$ & $\begin{array}{l}19.38 \\
18 j 2 . \\
4.986\end{array}$ & $\begin{array}{l}19.74 \\
1849 . \\
5.018\end{array}$ & $\begin{array}{l}20.09 \\
1865 \\
5.051\end{array}$ & $\begin{array}{l}20.44 \\
1882 \\
5.082\end{array}$ & $\begin{array}{l}20.78 \\
1898 \\
5.113\end{array}$ & $\begin{array}{l}21.12 \\
1914 . \\
5.143\end{array}$ & $\begin{array}{l}21.45 \\
1930 \\
5.173\end{array}$ & $\begin{array}{l}21.78 \\
1945 \\
5.203\end{array}$ & $\begin{array}{l}22.11 \\
1961 . \\
5.232\end{array}$ & $\begin{array}{l}22.03 \\
1977 . \\
5.260\end{array}$ \\
\hline
\end{tabular}


Table 10 (Continued)

Temperature range: 225 to $275^{\circ} \mathrm{C}$

\begin{tabular}{|c|c|c|c|c|c|c|c|c|c|c|c|c|}
\hline $\mathbf{p}$ & $\mathbf{T}$ & 225 & 230 & 235 & 240 & 245 & 250 & 255 & 260 & 265 & 270 & 275 \\
\hline 10700 & $\begin{array}{l}\text { D } \\
\text { is } \\
\text { s }\end{array}$ & $\begin{array}{l}18.80 \\
1874 . \\
4.945\end{array}$ & $\begin{array}{l}19.16 \\
1831 . \\
4.978\end{array}$ & $\begin{array}{l}19.52 \\
1847 \\
5.011\end{array}$ & $\begin{array}{l}19.87 \\
1864 . \\
5.049\end{array}$ & $\begin{array}{l}20.21 \\
1880 . \\
5.075\end{array}$ & $\begin{array}{l}20.55 \\
1896 . \\
5.106\end{array}$ & $\begin{array}{l}20.89 \\
1912 \\
5.137\end{array}$ & $\begin{array}{l}21.22 \\
1928 \\
5.167\end{array}$ & $\begin{array}{l}21.55 \\
1944 . \\
5.196\end{array}$ & $\begin{array}{l}21.87 \\
1960 . \\
5.225\end{array}$ & $\begin{array}{l}22.19 \\
1975 . \\
5.256\end{array}$ \\
\hline 10800 & $\begin{array}{l}\mathrm{y} \\
\mathrm{H} \\
\mathrm{s}\end{array}$ & $\begin{array}{l}18.59 \\
1812 \\
4.937\end{array}$ & $\begin{array}{l}18.95 \\
1829 \\
4.971\end{array}$ & $\begin{array}{l}19.30 \\
1846 . \\
5.004\end{array}$ & $\begin{array}{l}19.65 \\
1862 . \\
5.037\end{array}$ & $\begin{array}{l}19.99 \\
1879 . \\
5.068\end{array}$ & $\begin{array}{l}20.33 \\
7895 . \\
5.100\end{array}$ & $\begin{array}{l}20.66 \\
1911 \\
5.130\end{array}$ & $\begin{array}{l}20.99 \\
1927 . \\
5.160\end{array}$ & $\begin{array}{l}21.32 \\
1943 . \\
5.190\end{array}$ & $\begin{array}{l}21.64 \\
1958 . \\
5.219\end{array}$ & $\begin{array}{l}21.96 \\
1974 . \\
5.247\end{array}$ \\
\hline 10900 & $\begin{array}{l}\text { D } \\
\text { s } \\
\text { s }\end{array}$ & $\begin{array}{l}18.38 \\
1810 . \\
4.930\end{array}$ & $\begin{array}{l}18.74 \\
1827 . \\
4.964\end{array}$ & $\begin{array}{l}19.09 \\
1844 \\
4.997\end{array}$ & $\begin{array}{l}19.43 \\
1861 . \\
5.030\end{array}$ & $\begin{array}{l}19.77 \\
1877 . \\
5.062\end{array}$ & $\begin{array}{l}20.11 \\
1893 . \\
5.093\end{array}$ & $\begin{array}{l}20.44 \\
1909 . \\
5.123\end{array}$ & $\begin{array}{l}20.77 \\
1925 . \\
5.154\end{array}$ & $\begin{array}{l}21.09 \\
1941 . \\
5.183\end{array}$ & $\begin{array}{l}21.41 \\
1957 . \\
5.212\end{array}$ & $\begin{array}{l}21.73 \\
1973 . \\
5.241\end{array}$ \\
\hline 11000 & $\begin{array}{l}\text { : } \\
\text { ù }\end{array}$ & $\begin{array}{l}18.17 \\
1809 . \\
4.923\end{array}$ & $\begin{array}{l}18.53 \\
9826 . \\
9.957\end{array}$ & $\begin{array}{l}18.88 \\
1843 . \\
4.990\end{array}$ & $\begin{array}{l}19.22 \\
1859 . \\
5.023\end{array}$ & $\begin{array}{l}19.56 \\
1876 . \\
5.055\end{array}$ & $\begin{array}{l}19.89 \\
1892 . \\
5.086\end{array}$ & $\begin{array}{l}20.22 \\
1908 \\
5.117\end{array}$ & $\begin{array}{l}20.55 \\
1924 \\
5.147\end{array}$ & $\begin{array}{l}20.87 \\
1940 \\
5.177\end{array}$ & $\begin{array}{l}21.19 \\
1956 . \\
5.20 \epsilon\end{array}$ & $\begin{array}{l}21.51 \\
1971 \\
5.235\end{array}$ \\
\hline 11100 & $\begin{array}{l}y \\
\text { s } \\
s\end{array}$ & $\begin{array}{l}17.97 \\
1807 \\
4.916\end{array}$ & $\begin{array}{l}18.32 \\
1824 \\
4.950\end{array}$ & $\begin{array}{l}18.67 \\
1841 \\
4.984\end{array}$ & $\begin{array}{l}19.01 \\
1858_{-} \\
5.016\end{array}$ & $\begin{array}{l}19.35 \\
1874 \\
5.048\end{array}$ & $\begin{array}{l}19.68 \\
1890 . \\
5.080\end{array}$ & $\begin{array}{l}20.01 \\
1907 \\
5.110\end{array}$ & $\begin{array}{l}20.33 \\
1923 \\
5.141\end{array}$ & $\begin{array}{l}20.65 \\
1939 \\
5.170\end{array}$ & $\begin{array}{l}20.97 \\
1954 . \\
5.200\end{array}$ & $\begin{array}{l}21.29 \\
1970 . \\
5.228\end{array}$ \\
\hline 11200 & $\begin{array}{l}\text { V } \\
\text { B } \\
\text { S }\end{array}$ & $\begin{array}{l}77.77 \\
1805- \\
4.909\end{array}$ & $\begin{array}{l}18.12 \\
1822 . \\
4.943\end{array}$ & $\begin{array}{l}18.47 \\
1839 \\
4.977\end{array}$ & $\begin{array}{l}18.81 \\
1856 . \\
5.009\end{array}$ & $\begin{array}{l}19.14 \\
1873 \\
5.042\end{array}$ & $\begin{array}{l}19.47 \\
1889 . \\
5.073\end{array}$ & $\begin{array}{l}19.80 \\
1905 . \\
5.104\end{array}$ & $\begin{array}{l}20.12 \\
1921 . \\
5.134\end{array}$ & $\begin{array}{l}20.44 \\
1937 . \\
5.164\end{array}$ & $\begin{array}{l}20.76 \\
1953 . \\
5.193\end{array}$ & $\begin{array}{l}21.07 \\
1969 \\
5.222\end{array}$ \\
\hline 11300 & $\begin{array}{l}\text { D } \\
\text { H } \\
\text { S }\end{array}$ & $\begin{array}{l}17.58 \\
1803 . \\
4.902\end{array}$ & $\begin{array}{l}17.93 \\
1821 . \\
4.936\end{array}$ & $\begin{array}{l}18.27 \\
1838 \\
4.970\end{array}$ & $\begin{array}{l}18.61 \\
1854 . \\
5.003\end{array}$ & $\begin{array}{l}18.94 \\
1871 . \\
5.035\end{array}$ & $\begin{array}{l}19.27 \\
1987 . \\
5.066\end{array}$ & $\begin{array}{l}19.59 \\
1904 \\
5.097\end{array}$ & $\begin{array}{l}19.91 \\
1920 . \\
5.128\end{array}$ & $\begin{array}{l}20.23 \\
1936 . \\
5.158\end{array}$ & $\begin{array}{l}20.54 \\
1952 \\
5.187\end{array}$ & $\begin{array}{l}20.86 \\
1967 \\
5.26\end{array}$ \\
\hline 11400 & $\begin{array}{l}\text { V } \\
\text { B } \\
\text { S }\end{array}$ & $\begin{array}{l}17.39 \\
1802 . \\
4.895\end{array}$ & $\begin{array}{l}17.73 \\
1819 . \\
4.929\end{array}$ & $\begin{array}{l}18.07 \\
1836 . \\
4.963\end{array}$ & $\begin{array}{l}18.41 \\
1853 . \\
4.996\end{array}$ & $\begin{array}{l}18.74 \\
1869 . \\
5.028\end{array}$ & $\begin{array}{l}19.07 \\
1886 . \\
5.060\end{array}$ & $\begin{array}{l}19.39 \\
1902 . \\
5.091\end{array}$ & $\begin{array}{l}19.71 \\
1918 . \\
5.121\end{array}$ & $\begin{array}{l}20.03 \\
1934 . \\
5.15 i\end{array}$ & $\begin{array}{l}20.34 \\
3950 . \\
5.181\end{array}$ & $\begin{array}{l}20.65 \\
1966 . \\
5.210\end{array}$ \\
\hline 11500 & $\begin{array}{l}y \\
y \\
s\end{array}$ & $\begin{array}{l}17.20 \\
1800 . \\
4.888\end{array}$ & $\begin{array}{l}17.54 \\
1817 . \\
4.922\end{array}$ & $\begin{array}{l}17.88 \\
1834 . \\
4.956\end{array}$ & $\begin{array}{l}18.22 \\
1851 . \\
4.989\end{array}$ & $\begin{array}{l}18.55 \\
1868 . \\
5.022\end{array}$ & $\begin{array}{l}18.87 \\
1884 \\
5.053\end{array}$ & $\begin{array}{l}19.19 \\
1901 . \\
5.085\end{array}$ & $\begin{array}{l}19.51 \\
1917 . \\
5.115\end{array}$ & $\begin{array}{l}19.82 \\
1933 . \\
5.145\end{array}$ & $\begin{array}{l}20.13 \\
1949 . \\
5.175\end{array}$ & $\begin{array}{l}20.40 \\
1965 . \\
5.200\end{array}$ \\
\hline 11600 & $\begin{array}{l}\text { V } \\
\text { a } \\
\text { s }\end{array}$ & $\begin{array}{l}17.01 \\
1798 . \\
4.881\end{array}$ & $\begin{array}{l}17.36 \\
1816 \\
4.916\end{array}$ & $\begin{array}{l}17.69 \\
1833 \\
4.950\end{array}$ & $\begin{array}{l}18.03 \\
1850 . \\
4.983\end{array}$ & $\begin{array}{l}18.36 \\
1866 . \\
5.015\end{array}$ & $\begin{array}{l}18.68 \\
1883 \\
5.047\end{array}$ & $\begin{array}{l}19.00 \\
1899 . \\
5.078\end{array}$ & $\begin{array}{l}19.31 \\
1916 . \\
5.109\end{array}$ & $\begin{array}{l}19.62 \\
1932 \\
5.139\end{array}$ & $\begin{array}{l}19.93 \\
1948 . \\
5.168\end{array}$ & $\begin{array}{l}20.24 \\
1964 . \\
5.198\end{array}$ \\
\hline 11700 & $\begin{array}{l}y \\
y \\
\text { s }\end{array}$ & $\begin{array}{l}16.83 \\
1796 \\
4.874\end{array}$ & $\begin{array}{l}17.17 \\
1814 . \\
4.909\end{array}$ & $\begin{array}{l}17.51 \\
1831 \\
4.943\end{array}$ & $\begin{array}{l}17.84 \\
1848 . \\
4.976\end{array}$ & $\begin{array}{l}18.17 \\
1865 . \\
5.009\end{array}$ & $\begin{array}{l}18.49 \\
1881 . \\
5.041\end{array}$ & $\begin{array}{l}18.81 \\
1998 . \\
5.072\end{array}$ & $\begin{array}{l}19.12 \\
1914 . \\
5.103\end{array}$ & $\begin{array}{l}19.43 \\
1930 \\
5.133\end{array}$ & $\begin{array}{l}19.74 \\
1946 . \\
5.162\end{array}$ & $\begin{array}{l}20.04 \\
1962 \\
5.192\end{array}$ \\
\hline 11800 & $\begin{array}{l}y \\
y \\
s\end{array}$ & $\begin{array}{l}16.65 \\
1795 \\
4.867\end{array}$ & $\begin{array}{l}16.99 \\
1812 . \\
4.902\end{array}$ & $\begin{array}{l}17.33 \\
1829 . \\
4.936\end{array}$ & $\begin{array}{l}17.66 \\
1886 . \\
4.970\end{array}$ & $\begin{array}{l}17.98 \\
1863 . \\
5.002\end{array}$ & $\begin{array}{l}18.30 \\
1880 . \\
5.034\end{array}$ & $\begin{array}{l}18.62 \\
1986 . \\
5.066\end{array}$ & $\begin{array}{l}18.93 \\
1913 . \\
5.096\end{array}$ & $\begin{array}{l}19.24 \\
1929 \\
5.127\end{array}$ & $\begin{array}{l}19.54 \\
19.45 . \\
5.156\end{array}$ & $\begin{array}{l}19.84 \\
1961 . \\
5.185\end{array}$ \\
\hline 1900 & $\begin{array}{l}\text { D } \\
\text { g } \\
\text { S }\end{array}$ & $\begin{array}{l}16.47 \\
1793 . \\
4.860\end{array}$ & $\begin{array}{l}96.81 \\
1810 . \\
4.895\end{array}$ & $\begin{array}{l}17.15 \\
1828 . \\
4.930\end{array}$ & $\begin{array}{l}17.48 \\
1845 . \\
4.963\end{array}$ & $\begin{array}{l}17.80 \\
1862 . \\
4.996\end{array}$ & $\begin{array}{l}18.12 \\
1878 . \\
5.028\end{array}$ & $\begin{array}{l}18.43 \\
1895 . \\
5.059\end{array}$ & $\begin{array}{l}18.74 \\
1911 . \\
5.090\end{array}$ & $\begin{array}{l}19.05 \\
1928 . \\
5.120\end{array}$ & $\begin{array}{l}19.35 \\
1944 . \\
5.150\end{array}$ & $\begin{array}{l}19.65 \\
1960 . \\
5.180\end{array}$ \\
\hline
\end{tabular}


Table 10 (Continued)

Temperature range: 225 to $275^{\circ} \mathrm{C}$

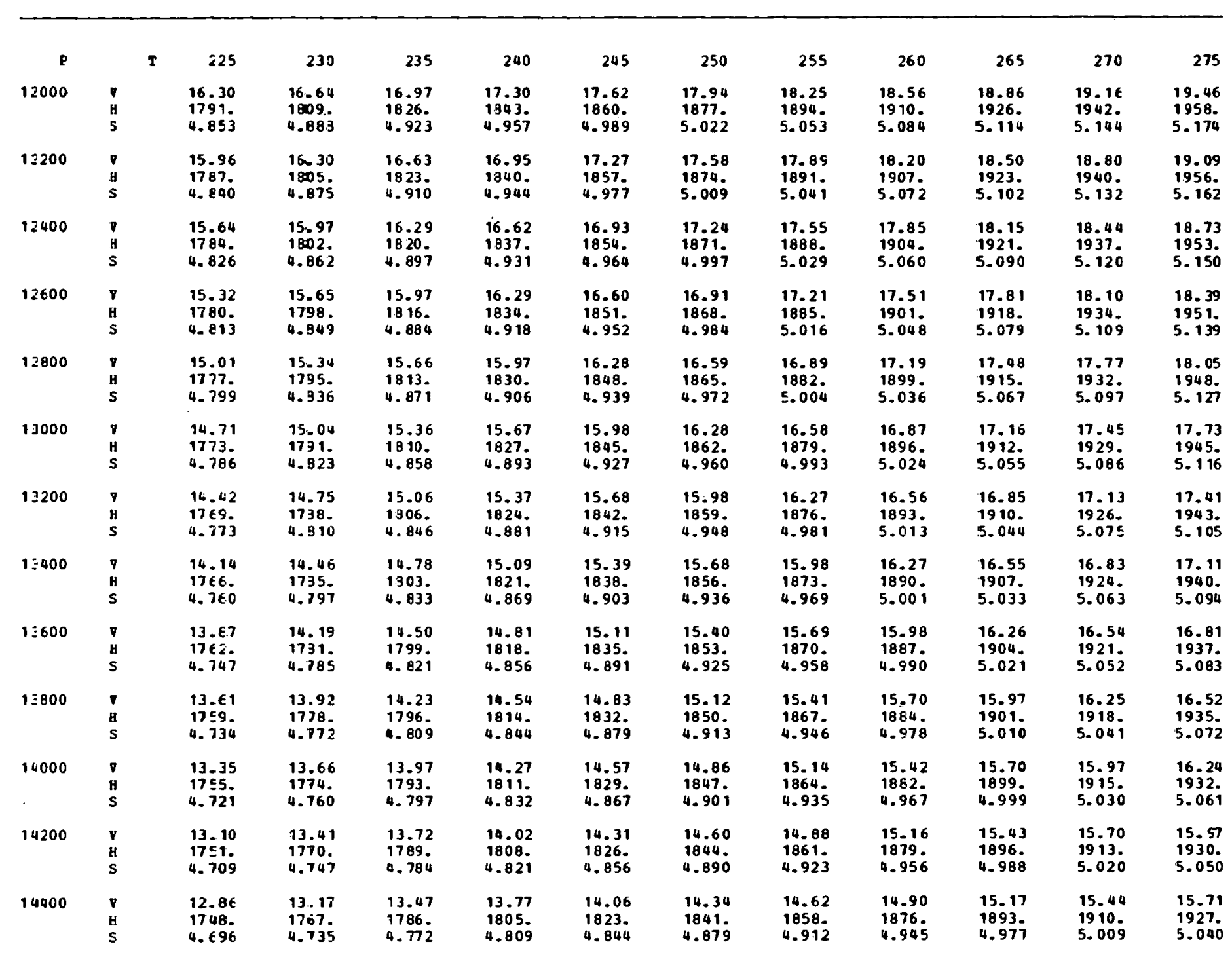


Table 10 (Continued)

Temperature range: 225 to $275^{\circ} \mathrm{C}$

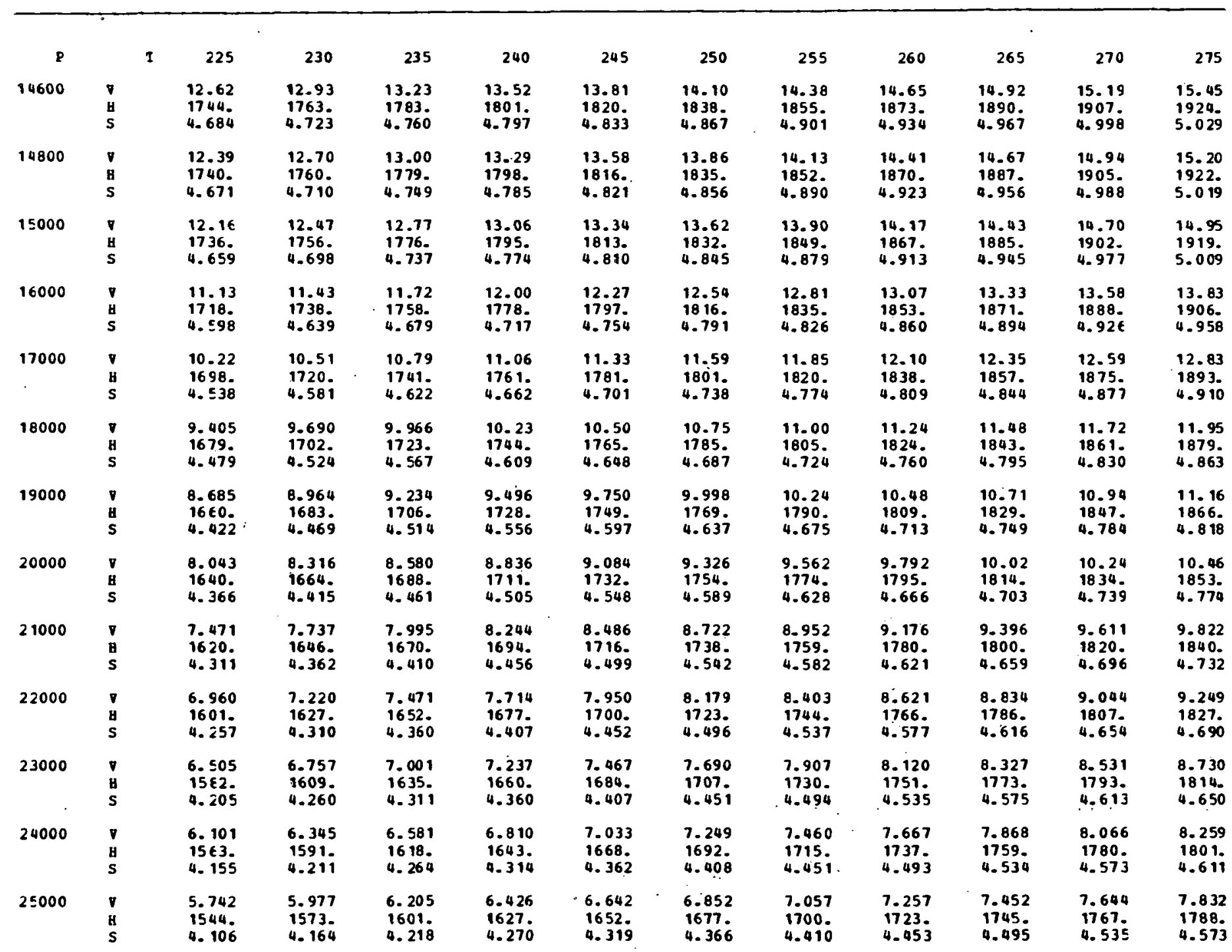


Table 0 (Continued)

Temperature range: 225 to $275^{\circ} \mathrm{C}$

\begin{tabular}{|c|c|c|c|c|c|c|c|c|c|c|c|c|c|}
\hline $\mathbf{P}$ & & $\mathbf{I}$ & 225 & 230 & 235 & 240 & 245 & 250 & 255 & 260 & 265 & 270 & 275 \\
\hline 25000 & $\begin{array}{l}\nabla \\
\text { y } \\
\text { S }\end{array}$ & & $\begin{array}{l}5.423 \\
1527 . \\
4.059\end{array}$ & $\begin{array}{l}5.649 \\
1556 . \\
4.118\end{array}$ & $\begin{array}{l}5.868 \\
1584 . \\
4.174\end{array}$ & $\begin{array}{l}6.082 \\
1611 . \\
4.227\end{array}$ & $\begin{array}{l}6.290 \\
1637 . \\
4.277\end{array}$ & $\begin{array}{l}6.493 \\
1662 . \\
4.325\end{array}$ & $\begin{array}{l}6.692 \\
1686 . \\
4.370\end{array}$ & $\begin{array}{l}6.885 \\
1710 . \\
4.414\end{array}$ & $\begin{array}{l}7.075 \\
1732 \\
4.457\end{array}$ & $\begin{array}{l}7.261 \\
1754 . \\
4.497\end{array}$ & $\begin{array}{l}7.443 \\
1776 . \\
4.537\end{array}$ \\
\hline 29000 & $\begin{array}{l}y \\
H \\
S\end{array}$ & & $\begin{array}{l}4.891 \\
1494 . \\
3.972\end{array}$ & $\begin{array}{l}5097 \\
524 . \\
4.033\end{array}$ & $\begin{array}{l}5.298 \\
1553 . \\
4.091\end{array}$ & $\begin{array}{l}5.496 \\
1581 . \\
4.146\end{array}$ & $\begin{array}{l}5.689 \\
1608 . \\
4.198\end{array}$ & $\begin{array}{l}5.878 \\
1634 . \\
4.247\end{array}$ & $\begin{array}{l}6.063 \\
1659 . \\
4.295\end{array}$ & $\begin{array}{l}6.244 \\
1683 . \\
4.340\end{array}$ & $\begin{array}{l}6.422 \\
1706 . \\
4.384\end{array}$ & $\begin{array}{l}6.596 \\
1729 . \\
4.426\end{array}$ & $\begin{array}{l}6.767 \\
1751 \\
4.467\end{array}$ \\
\hline 30000 & $\begin{array}{l}\mathbf{V} \\
\mathbf{H} \\
\mathbf{S}\end{array}$ & & $\begin{array}{l}4.470 \\
14 \notin 4 . \\
3.893\end{array}$ & $\begin{array}{l}6.657 \\
2495 . \\
3.955\end{array}$ & $\begin{array}{l}4.841 \\
1525 . \\
4.014\end{array}$ & $\begin{array}{l}5.022 \\
1553 . \\
4.071\end{array}$ & $\begin{array}{l}5.201 \\
1581 . \\
4.124\end{array}$ & $\begin{array}{l}5.376 \\
1608 . \\
4.175\end{array}$ & $\begin{array}{l}5.547 \\
1633 . \\
4.224\end{array}$ & $\begin{array}{l}5.716 \\
1658 . \\
4.271\end{array}$ & $\begin{array}{l}5.882 \\
1682 . \\
4.316\end{array}$ & $\begin{array}{l}6.045 \\
1706 . \\
4.359\end{array}$ & $\begin{array}{l}6.205 \\
1728 . \\
4.401\end{array}$ \\
\hline 32000 & $\begin{array}{l}\text { I } \\
H \\
\text { S }\end{array}$ & & $\begin{array}{l}4.135 \\
1437 \\
3.822\end{array}$ & $\begin{array}{l}\text { 4. } 304 \\
t 468 \text {. } \\
3.884\end{array}$ & $\begin{array}{l}4.471 \\
1498 . \\
3.944\end{array}$ & $\begin{array}{l}4.637 \\
1528 . \\
4.002\end{array}$ & $\begin{array}{l}4.801 \\
1556 . \\
4.056\end{array}$ & $\begin{array}{l}4.963 \\
1583 . \\
4.108\end{array}$ & $\begin{array}{l}5.122 \\
1609 . \\
4.158\end{array}$ & $\begin{array}{l}5.279 \\
1635 . \\
4.206\end{array}$ & $\begin{array}{l}5.433 \\
1659 . \\
4.252\end{array}$ & $\begin{array}{l}5.585 \\
1683 \\
4.297\end{array}$ & $\begin{array}{l}5.735 \\
1707 \\
4.340\end{array}$ \\
\hline 34000 & $\begin{array}{l}\mathbf{V} \\
\mathbf{H} \\
\mathbf{S}\end{array}$ & & $\begin{array}{l}3.866 \\
14.33 \\
3.758\end{array}$ & $\begin{array}{l}4.018 \\
1444 . \\
1.821\end{array}$ & $\begin{array}{l}4.169 \\
1475 \\
3.881\end{array}$ & $\begin{array}{l}4.321 \\
1504 . \\
3.938\end{array}$ & $\begin{array}{l}4.472 \\
1533 . \\
3.994\end{array}$ & $\begin{array}{l}4.621 \\
1560 \\
4.047\end{array}$ & $\begin{array}{l}4.769 \\
1587 \\
4.097\end{array}$ & $\begin{array}{l}4.915 \\
1613 . \\
4.146\end{array}$ & $\begin{array}{l}5.058 \\
1638 \\
0.193\end{array}$ & $\begin{array}{l}5.200 \\
1662 . \\
4.239\end{array}$ & $\begin{array}{l}5.340 \\
1686 . \\
4.282\end{array}$ \\
\hline 36000 & $\begin{array}{l}\mathbf{V} \\
\mathrm{H} \\
\mathbf{S}\end{array}$ & & $\begin{array}{l}3.648 \\
1392 . \\
3.701\end{array}$ & $\begin{array}{l}3.784 \\
1423 . \\
3.763\end{array}$ & $\begin{array}{l}3.921 \\
1453 \\
3.823\end{array}$ & $\begin{array}{l}4.059 \\
1483 \\
3.880\end{array}$ & $\begin{array}{l}4.198 \\
1511 . \\
3.936\end{array}$ & $\begin{array}{l}4.335 \\
1539 . \\
3.989\end{array}$ & $\begin{array}{l}4.472 \\
1566 \\
4.041\end{array}$ & $\begin{array}{l}4.608 \\
1593 \\
4.090\end{array}$ & $\begin{array}{l}4.742 \\
1618 . \\
4.138\end{array}$ & $\begin{array}{l}4.879 \\
1643 \\
4.184\end{array}$ & $\begin{array}{l}5.005 \\
1667 \\
4.228\end{array}$ \\
\hline 38000 & $\begin{array}{l}\mathbf{V} \\
\mathbf{H} \\
\mathbf{S}\end{array}$ & & $\begin{array}{l}3.470 \\
1374 \\
3.651\end{array}$ & $\begin{array}{l}3.591 \\
1404 . \\
3.711\end{array}$ & $\begin{array}{l}3.715 \\
1434 . \\
3.770\end{array}$ & $\begin{array}{l}3.841 \\
1963 . \\
3.828\end{array}$ & $\begin{array}{l}3.967 \\
1492 . \\
3.883\end{array}$ & $\begin{array}{l}4.094 \\
1520 \\
3.937\end{array}$ & $\begin{array}{l}4.221 \\
1547 \\
3.988\end{array}$ & $\begin{array}{l}4.347 \\
1574 \\
4.038\end{array}$ & $\begin{array}{l}4.472 \\
1600 \\
4.087\end{array}$ & $\begin{array}{l}4.596 \\
1625 . \\
4.133\end{array}$ & $\begin{array}{l}4.719 \\
1649 . \\
4.178\end{array}$ \\
\hline 40000 & $\begin{array}{l}V \\
y \\
S\end{array}$ & & $\begin{array}{l}3.323 \\
1358 . \\
3.606\end{array}$ & $\begin{array}{l}1.432 \\
1388 \\
1.665\end{array}$ & $\begin{array}{l}3.543 \\
1417 . \\
3.723\end{array}$ & $\begin{array}{l}3.657 \\
1446 \\
3.779\end{array}$ & $\begin{array}{l}3.773 \\
1475 . \\
3.834\end{array}$ & $\begin{array}{l}3.890 \\
1502 . \\
3.888\end{array}$ & $\begin{array}{l}4.007 \\
1530 \\
3.940\end{array}$ & $\begin{array}{l}4.124 \\
1556 \\
3.990\end{array}$ & $\begin{array}{l}4.241 \\
1582 \\
4.038\end{array}$ & $\begin{array}{l}4.357 \\
1608 \\
4.085\end{array}$ & $\begin{array}{l}4.472 \\
1632 \\
4.131\end{array}$ \\
\hline 42000 & $\begin{array}{l}y \\
y \\
s\end{array}$ & & $\begin{array}{l}3.201 \\
13455 \\
3.565\end{array}$ & $\begin{array}{l}3.298 \\
2374 . \\
\equiv 623\end{array}$ & $\begin{array}{l}3.399 \\
1402 . \\
3.680\end{array}$ & $\begin{array}{l}3.502 \\
1431 . \\
3.735\end{array}$ & $\begin{array}{l}3.607 \\
1459 . \\
3.790\end{array}$ & $\begin{array}{l}3.715 \\
1487 . \\
3.843\end{array}$ & $\begin{array}{l}3.823 \\
1514 . \\
3.894\end{array}$ & $\begin{array}{l}3.932 \\
1540 . \\
3.945\end{array}$ & $\begin{array}{l}4.041 \\
1566 . \\
3.993\end{array}$ & $\begin{array}{l}4.150 \\
1592 . \\
4.040\end{array}$ & $\begin{array}{l}4.258 \\
1617 . \\
4.086\end{array}$ \\
\hline 44000 & $\begin{array}{l}\nabla \\
8 \\
S\end{array}$ & & $\begin{array}{l}3.098 \\
1333 \\
3.529\end{array}$ & $\begin{array}{l}3.185 \\
1361 \\
3.586\end{array}$ & $\begin{array}{l}3.276 \\
1389 \\
3.64 i\end{array}$ & $\begin{array}{l}3.370 \\
1417 \\
3.696\end{array}$ & $\begin{array}{l}3.466 \\
1445 \\
3.749\end{array}$ & $\begin{array}{l}3.564 \\
1472 . \\
3.801\end{array}$ & $\begin{array}{l}3.660 \\
1499 \\
3.853\end{array}$ & $\begin{array}{l}3.765 \\
1526 \\
3.903\end{array}$ & $\begin{array}{l}3.867 \\
1552 \\
3.951\end{array}$ & $\begin{array}{l}3.969 \\
1577 \\
3.998\end{array}$ & $\begin{array}{l}4.071 \\
1602 \\
4.044\end{array}$ \\
\hline $4 E 000$ & $\begin{array}{l}\mathbf{D} \\
H \\
S\end{array}$ & & $\begin{array}{l}3.009 \\
1323 . \\
3.496\end{array}$ & $\begin{array}{l}3.089 \\
1351 \\
3.552\end{array}$ & $\begin{array}{l}3.172 \\
1378 . \\
3.606\end{array}$ & $\begin{array}{l}3.257 \\
1405 \\
3.659\end{array}$ & $\begin{array}{l}3.345 \\
1432 . \\
3.712\end{array}$ & $\begin{array}{l}3.435 \\
1459 . \\
3.763\end{array}$ & $\begin{array}{l}3.527 \\
1486 . \\
3.814\end{array}$ & $\begin{array}{l}3.621 \\
1512 \\
3.863\end{array}$ & $\begin{array}{l}3.715 \\
1538 \\
3.912\end{array}$ & $\begin{array}{l}3.890 \\
1563 . \\
3.959\end{array}$ & $\begin{array}{l}3.906 \\
1588 . \\
4.005\end{array}$ \\
\hline 43000 & $\begin{array}{l}\nabla \\
H \\
S\end{array}$ & & $\begin{array}{l}2.933 \\
1314 . \\
3.466\end{array}$ & $\begin{array}{l}3.006 \\
1341 . \\
1520\end{array}$ & $\begin{array}{l}3.082 \\
1368 . \\
3.574\end{array}$ & $\begin{array}{l}3.160 \\
1395 . \\
3.626\end{array}$ & $\begin{array}{l}3.240 \\
1421 . \\
3.678\end{array}$ & $\begin{array}{l}3.323 \\
1448 . \\
3.728\end{array}$ & $\begin{array}{l}3.408 \\
1474 \\
3.778\end{array}$ & $\begin{array}{l}3.490 \\
1500 \\
3.827\end{array}$ & $\begin{array}{l}3.582 \\
1526 \\
3.875\end{array}$ & $\begin{array}{l}3.671 \\
1551 \\
3.922\end{array}$ & $\begin{array}{l}3.760 \\
1576 . \\
3.968\end{array}$ \\
\hline 50000 & $\begin{array}{l}V \\
H \\
S\end{array}$ & & $\begin{array}{l}2.866 \\
1306 . \\
3.439\end{array}$ & $\begin{array}{l}2.934 \\
1333 . \\
3.492\end{array}$ & $\begin{array}{l}3.003 \\
1359 . \\
3.544\end{array}$ & $\begin{array}{l}3.075 \\
1385 . \\
3.596\end{array}$ & $\begin{array}{l}3.149 \\
1411 . \\
3.646\end{array}$ & $\begin{array}{l}3.225 \\
1437 . \\
3.696\end{array}$ & $\begin{array}{l}3.303 \\
1463 . \\
3.745\end{array}$ & $\begin{array}{l}3.383 \\
1489 \\
3.794\end{array}$ & $\begin{array}{l}3.465 \\
1514 . \\
3.891\end{array}$ & $\begin{array}{l}3.548 \\
1539 . \\
3.888\end{array}$ & $\begin{array}{l}3.632 \\
1564 . \\
3.933\end{array}$ \\
\hline
\end{tabular}


Table 10 (Continued)

Temperature range: 280 to $330^{\circ} \mathrm{C}$

\begin{tabular}{|c|c|c|c|c|c|c|c|c|c|c|c|c|}
\hline P & I & 280 & 285 & 290 & 295 & 300 & 305 & 310 & 315 & 320 & 325 & 330 \\
\hline 5 & $\begin{array}{l}\nabla \\
\mathbf{u} \\
\mathbf{S}\end{array}$ & $\begin{array}{r}54000 . \\
2125 \\
9.217\end{array}$ & $\begin{array}{r}54490 . \\
2138 . \\
5.240\end{array}$ & $\begin{array}{r}54980 . \\
2150 . \\
9.263\end{array}$ & $\begin{array}{r}55470 . \\
2163 . \\
9.286\end{array}$ & $\begin{array}{r}55950 . \\
2176 . \\
9.308\end{array}$ & $\begin{array}{r}56440 \\
2189 \\
9.331\end{array}$ & $\begin{array}{r}56930 . \\
2202 \\
9.353\end{array}$ & $\begin{array}{c}57420 \\
2215 \\
9.376\end{array}$ & $\begin{array}{r}57910 . \\
2229 . \\
9.398\end{array}$ & $\begin{array}{r}58390 \\
2242 . \\
9.420\end{array}$ & $\begin{array}{l}58880 . \\
2255 . \\
9.442\end{array}$ \\
\hline 10 & $\begin{array}{l}\mathbf{D} \\
\mathbf{H} \\
\mathbf{S}\end{array}$ & $\begin{array}{r}27000 \\
2125 \\
8.878\end{array}$ & $\begin{array}{r}27240 \\
2138 \\
8.901\end{array}$ & $\begin{array}{r}27490 \\
2150 \\
8.924\end{array}$ & $\begin{array}{r}27730 . \\
2163 . \\
8.947\end{array}$ & $\begin{array}{r}27980 \\
2176 \\
8.970\end{array}$ & $\begin{array}{r}28220 \\
2189 \\
8.993\end{array}$ & $\begin{array}{r}28460 \\
2202 \\
9.015\end{array}$ & $\begin{array}{r}28710 \\
2215 \\
9.037\end{array}$ & $\begin{array}{r}28950 \\
2228 \\
9.060\end{array}$ & $\begin{array}{r}29200 \\
2242 \\
9.082\end{array}$ & $\begin{array}{r}29040 . \\
2255 . \\
9.104\end{array}$ \\
\hline 50 & $\begin{array}{l}8 \\
y \\
\text { S }\end{array}$ & $\begin{array}{l}5400 . \\
2124 . \\
8.092\end{array}$ & $\begin{array}{l}5450 . \\
2137 . \\
8.115\end{array}$ & $\begin{array}{l}5500 . \\
2150 . \\
8.138\end{array}$ & $\begin{array}{l}5540 . \\
2163 \\
8.16 i\end{array}$ & $\begin{array}{l}5590 \\
2176 \\
8.184\end{array}$ & $\begin{array}{l}5640 . \\
2189 . \\
8.206\end{array}$ & $\begin{array}{l}5690 . \\
2202 . \\
8.229\end{array}$ & $\begin{array}{l}5740 . \\
2215 \\
8.251\end{array}$ & $\begin{array}{l}5790 . \\
2228 \\
8.273\end{array}$ & $\begin{array}{l}5840 . \\
2241 . \\
8.295\end{array}$ & $\begin{array}{l}5890 . \\
2254 . \\
8.317\end{array}$ \\
\hline 100 & $\begin{array}{l}\text { Y } \\
\text { H } \\
\text { S }\end{array}$ & $\begin{array}{l}2700 \\
2124 \\
7.753\end{array}$ & $\begin{array}{l}2720 . \\
2136 \\
7.776\end{array}$ & $\begin{array}{l}2750 . \\
2149 . \\
7.799\end{array}$ & $\begin{array}{l}2770 . \\
2162 . \\
7.822\end{array}$ & $\begin{array}{l}2800 . \\
2175 . \\
7.845\end{array}$ & $\begin{array}{l}2820 \\
2188 \\
7.867\end{array}$ & $\begin{array}{l}2840 . \\
2201 . \\
7.890\end{array}$ & $\begin{array}{l}2870 . \\
2214 . \\
7.912\end{array}$ & $\begin{array}{l}2890 \\
2228 \\
7.934\end{array}$ & $\begin{array}{l}2920 . \\
2241 . \\
7.956\end{array}$ & $\begin{array}{l}2940 . \\
2254 . \\
7.978\end{array}$ \\
\hline 101.33 & $\begin{array}{l}\mathbf{Y} \\
H \\
\text { S }\end{array}$ & $\begin{array}{l}26 \in 0 . \\
2124 . \\
7.746\end{array}$ & $\begin{array}{l}2690 . \\
2136 . \\
7.769\end{array}$ & $\begin{array}{l}2710 . \\
2149 . \\
7.792\end{array}$ & $\begin{array}{l}2730 . \\
2162 . \\
7.815\end{array}$ & $\begin{array}{l}2760 . \\
2175 . \\
7.838\end{array}$ & $\begin{array}{l}2780 \\
2188 \\
7.861\end{array}$ & $\begin{array}{l}2810 . \\
2201 \\
7.883\end{array}$ & $\begin{array}{l}2830 . \\
2214 . \\
7.906\end{array}$ & $\begin{array}{l}2860 \\
2227 \\
7.928\end{array}$ & $\begin{array}{l}2880 . \\
2241 . \\
7.950\end{array}$ & $\begin{array}{l}2900 . \\
2254 . \\
7.972\end{array}$ \\
\hline 200 & $\begin{array}{l}\mathbf{V} \\
\mathbf{H} \\
\mathbf{S}\end{array}$ & $\begin{array}{l}1350 . \\
2122 . \\
7.413\end{array}$ & $\begin{array}{l}1360 . \\
2135 . \\
7.436\end{array}$ & $\begin{array}{l}1370 . \\
2148 . \\
7.459\end{array}$ & $\begin{array}{l}1380 . \\
2161 . \\
7.482\end{array}$ & $\begin{array}{l}1400 . \\
2174 . \\
7.505\end{array}$ & $\begin{array}{l}1410 \\
2187 \\
7.527\end{array}$ & $\begin{array}{l}1420 . \\
2200 . \\
7.550\end{array}$ & $\begin{array}{l}1430 \\
2213 \\
7.572\end{array}$ & $\begin{array}{l}1450 . \\
2226 \\
7.594\end{array}$ & $\begin{array}{l}1460 \\
2240 \\
7.696\end{array}$ & $\begin{array}{l}1470 . \\
2253 . \\
7.638\end{array}$ \\
\hline 300 & $\begin{array}{l}\nabla \\
u \\
S\end{array}$ & $\begin{array}{l}897.6 \\
2121 . \\
7.213\end{array}$ & $\begin{array}{l}905.8 \\
2134 . \\
7.236\end{array}$ & $\begin{array}{l}914.0 \\
2147 . \\
7.259\end{array}$ & $\begin{array}{l}922.2 \\
2160 . \\
7.282\end{array}$ & $\begin{array}{l}930.5 \\
2173 . \\
7.305\end{array}$ & $\begin{array}{l}938.7 \\
2186 . \\
7.328\end{array}$ & $\begin{array}{l}946.9 \\
2199 . \\
7.350\end{array}$ & $\begin{array}{l}955.1 \\
2212 . \\
7.373\end{array}$ & $\begin{array}{l}963.3 \\
2225 . \\
7.395\end{array}$ & $\begin{array}{l}971.5 \\
2239.5 \\
7.417\end{array}$ & $\begin{array}{l}979.7 \\
2252 . \\
7.439\end{array}$ \\
\hline 400 & $\begin{array}{l}\mathbf{V} \\
\mathbf{H} \\
\mathbf{S}\end{array}$ & $\begin{array}{l}672.5 \\
2.320 . \\
7.071\end{array}$ & $\begin{array}{l}678.7 \\
2133 . \\
7.094\end{array}$ & $\begin{array}{l}684.9 \\
2146 . \\
7.117\end{array}$ & $\begin{array}{l}691.1 \\
2159 . \\
7.140\end{array}$ & $\begin{array}{l}697.3 \\
2172 . \\
7.163\end{array}$ & $\begin{array}{l}703.5 \\
2185 . \\
7.186\end{array}$ & $\begin{array}{l}709.7 \\
2198 . \\
7.208\end{array}$ & $\begin{array}{l}715.8 \\
2211 . \\
7.231\end{array}$ & $\begin{array}{l}722.0 \\
2224 . \\
7.253\end{array}$ & $\begin{array}{l}728.2 \\
2237 . \\
7.275\end{array}$ & $\begin{array}{l}734.4 \\
2251 . \\
7.297\end{array}$ \\
\hline 500 & $\begin{array}{l}y \\
\text { H } \\
\text { S }\end{array}$ & $\begin{array}{l}537.5 \\
2119 . \\
6.960\end{array}$ & $\begin{array}{l}542.5 \\
2132 . \\
6.983\end{array}$ & $\begin{array}{l}547.5 \\
2144 . \\
7.006\end{array}$ & $\begin{array}{l}55.2 .4 \\
2158 . \\
7.029\end{array}$ & $\begin{array}{l}557.4 \\
2171 . \\
7.052\end{array}$ & $\begin{array}{l}562.4 \\
2184 . \\
7.075\end{array}$ & $\begin{array}{l}567.3 \\
2197 . \\
7.098\end{array}$ & $\begin{array}{l}572.3 \\
2210 . \\
7.120\end{array}$ & $\begin{array}{l}577.3 \\
2223 . \\
7.142\end{array}$ & $\begin{array}{l}582.2 \\
2236 . \\
7.16 \leq\end{array}$ & $\begin{array}{l}587.2 \\
2250 . \\
7.487\end{array}$ \\
\hline 600 & $\begin{array}{l}\mathbf{y} \\
\mathrm{g} \\
\mathbf{s}\end{array}$ & $\begin{array}{l}447.5 \\
2177 . \\
6.869\end{array}$ & $\begin{array}{l}451.7 \\
2130 . \\
6.893\end{array}$ & $\begin{array}{l}455.8 \\
2143 . \\
6.916\end{array}$ & $\begin{array}{l}460.0 \\
2156 \\
6.939\end{array}$ & $\begin{array}{l}064.1 \\
.2169 . \\
6.962\end{array}$ & $\begin{array}{l}468.3 \\
2183 . \\
6.984\end{array}$ & $\begin{array}{l}472.4 \\
2196 \\
7.007\end{array}$ & $\begin{array}{l}476.6 \\
2209 \\
7.030\end{array}$ & $\begin{array}{l}980.7 \\
2222 . \\
7.052\end{array}$ & $\begin{array}{l}484.5 \\
2235 \\
7.070\end{array}$ & $\begin{array}{l}489.0 \\
2249 . \\
7.096\end{array}$ \\
\hline 700 & $\begin{array}{l}\nabla \\
\mathbf{H} \\
\mathbf{S}\end{array}$ & $\begin{array}{l}383.2 \\
2116 . \\
6.792\end{array}$ & $\begin{array}{l}386.8 \\
2129 . \\
6.816\end{array}$ & $\begin{array}{l}390.4 \\
2142 . \\
6.839\end{array}$ & $\begin{array}{l}394.0 \\
2155 . \\
6.862\end{array}$ & $\begin{array}{l}397 . \dot{5} \\
2168 . \\
6.885\end{array}$ & $\begin{array}{l}401.1 \\
2181 . \\
6.908\end{array}$ & $\begin{array}{l}404.7 \\
2195 . \\
6.930\end{array}$ & $\begin{array}{l}408.2 \\
2208 . \\
6.953\end{array}$ & $\begin{array}{l}411.8 \\
2221 . \\
6.975\end{array}$ & $\begin{array}{l}415.4 \\
2234 . \\
6.997\end{array}$ & $\begin{array}{l}418.9 \\
2248 . \\
7.020\end{array}$ \\
\hline 800 & $\begin{array}{l}\mathbf{V} \\
\mathbf{H} \\
\mathbf{S}\end{array}$ & $\begin{array}{l}335.0 \\
2115 \\
6.725\end{array}$ & $\begin{array}{l}338.2 \\
2128 . \\
6.749\end{array}$ & $\begin{array}{l}341.3 \\
2141 . \\
6.772\end{array}$ & $\begin{array}{l}344.4 \\
2154 . \\
6.795\end{array}$ & $\begin{array}{l}347.6 \\
21.67 \\
6.818\end{array}$ & $\begin{array}{l}350.7 \\
2180 . \\
6.841\end{array}$ & $\begin{array}{l}353.8 \\
2193 . \\
6.863\end{array}$ & $\begin{array}{l}357.0 \\
2207 . \\
6.886\end{array}$ & $\begin{array}{l}360.1 \\
2220 . \\
6.908\end{array}$ & $\begin{array}{l}363.2 \\
2233 . \\
6.931\end{array}$ & $\begin{array}{l}366.3 \\
2246 . \\
6.953\end{array}$ \\
\hline 900 & $\begin{array}{l}\mathbf{v} \\
\mathbf{y} \\
\mathbf{s}\end{array}$ & $\begin{array}{l}297.5 \\
2114 . \\
6.666\end{array}$ & $\begin{array}{l}300.3 \\
2127 . \\
6.689\end{array}$ & $\begin{array}{l}303.1 \\
2140 . \\
6.713\end{array}$ & $\begin{array}{l}305.9 \\
2153 . \\
6.736\end{array}$ & $\begin{array}{l}308.7 \\
2166 . \\
6.759\end{array}$ & $\begin{array}{l}311.5 \\
2179 . \\
6.782\end{array}$ & $\begin{array}{l}314.3 \\
2192 . \\
6.804\end{array}$ & $\begin{array}{r}317.1 \\
+2206 . \\
\end{array}$ & $\begin{array}{l}319.9 \\
2219 . \\
6.849\end{array}$ & $\begin{array}{l}322.7 \\
2232 . \\
6.872\end{array}$ & $\begin{array}{l}325.4 \\
2245 . \\
6.894\end{array}$ \\
\hline
\end{tabular}


Table :0 (Continued)

Temperature range: 280 to $330^{\circ} \mathrm{C}$

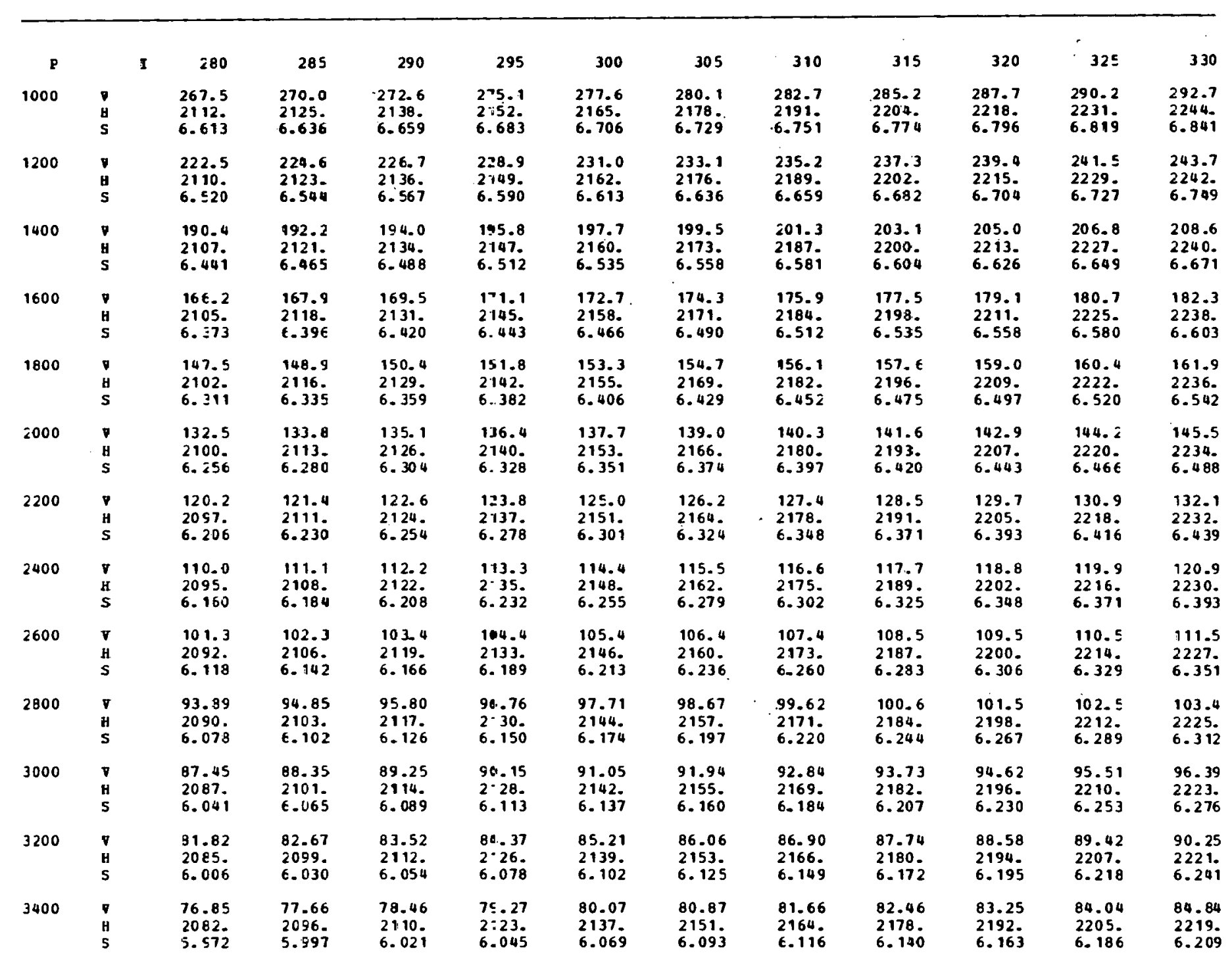


Table 10 (Continued)

Temperature range: 280 to $330^{\circ} \mathrm{C}$

\begin{tabular}{|c|c|c|c|c|c|c|c|c|c|c|c|c|}
\hline P & 1 & 280 & 285 & 290 & 295 & 300 & 305 & 310 & 315 & 320 & 325 & 330 \\
\hline 3600 & $\begin{array}{l}7 \\
\text { H } \\
5\end{array}$ & $\begin{array}{l}72.44 \\
2080 . \\
5.941\end{array}$ & $\begin{array}{l}73.20 \\
2094 . \\
5.965\end{array}$ & $\begin{array}{l}73.97 \\
2107 . \\
5.990\end{array}$ & $\begin{array}{l}74.73 \\
2121 . \\
6.014\end{array}$ & $\begin{array}{l}75.49 \\
2135 . \\
6.038\end{array}$ & $\begin{array}{l}76.25 \\
2148 . \\
6.062\end{array}$ & $\begin{array}{l}77.01 \\
2162 . \\
6.085\end{array}$ & $\begin{array}{l}77.76 \\
2176 . \\
6.109\end{array}$ & $\begin{array}{l}78.52 \\
2189 . \\
6.132\end{array}$ & $\begin{array}{l}79.27 \\
2203 \\
6.155\end{array}$ & $\begin{array}{l}80.02 \\
2217 \\
6.178\end{array}$ \\
\hline 3800 & $\begin{array}{l}\mathbf{v} \\
: \mathrm{H} \\
\mathrm{s}\end{array}$ & $\begin{array}{l}68.48 \\
2077 \\
5.911\end{array}$ & $\begin{array}{l}69.22 \\
2091 . \\
5.935\end{array}$ & $\begin{array}{l}69.94 \\
2105 . \\
5.960\end{array}$ & $\begin{array}{l}70.67 \\
2119 . \\
5.984\end{array}$ & $\begin{array}{l}71.40 \\
2132 . \\
6.008\end{array}$ & $\begin{array}{l}72.12 \\
2146 . \\
6.032\end{array}$ & $\begin{array}{l}72.84 \\
2160 . \\
6.056\end{array}$ & $\begin{array}{l}73.56 \\
2173 . \\
6.079\end{array}$ & $\begin{array}{l}74.28 \\
2187 \\
6.102\end{array}$ & $\begin{array}{l}74.99 \\
2201 . \\
6.126\end{array}$ & $\begin{array}{l}75.71 \\
2215 . \\
6.149\end{array}$ \\
\hline 4000 & $\begin{array}{l}\text { v } \\
\text { H } \\
\text { s }\end{array}$ & $\begin{array}{l}64.93 \\
2075 . \\
5.882\end{array}$ & $\begin{array}{l}65.63 \\
2089 \\
5.907\end{array}$ & $\begin{array}{l}66.32 \\
2102 . \\
5.931\end{array}$ & $\begin{array}{l}67.02 \\
2116 . \\
5.956\end{array}$ & $\begin{array}{l}67.71 \\
2130 . \\
5.980\end{array}$ & $\begin{array}{l}68.40 \\
2194 . \\
6.004\end{array}$ & $\begin{array}{l}69.09 \\
2157 \\
6.027\end{array}$ & $\begin{array}{l}69.78 \\
2171 . \\
6.05 i\end{array}$ & $\begin{array}{l}70.46 \\
2185 \\
6.074\end{array}$ & $\begin{array}{l}71.15 \\
2199 . \\
6.098\end{array}$ & $\begin{array}{l}71.83 \\
2213 \\
6.121\end{array}$ \\
\hline 4200 & $\begin{array}{l}\text { v } \\
\text { s } \\
\text { s }\end{array}$ & $\begin{array}{l}61.71 \\
2073 . \\
5-855\end{array}$ & $\begin{array}{l}62.38 \\
2086 . \\
5.880\end{array}$ & $\begin{array}{l}63.04 \\
2100 . \\
5.904\end{array}$ & $\begin{array}{l}63.71 \\
2114 . \\
5.929\end{array}$ & $\begin{array}{l}64.37 \\
2128 . \\
5.953\end{array}$ & $\begin{array}{l}65.04 \\
2241 . \\
5.977\end{array}$ & $\begin{array}{l}65.70 \\
2155 \\
6.001\end{array}$ & $\begin{array}{l}66.35 \\
2169 . \\
6.024\end{array}$ & $\begin{array}{l}67.01 \\
2183 . \\
6.048\end{array}$ & $\begin{array}{l}67.67 \\
2197 \\
6.071\end{array}$ & $\begin{array}{l}68.32 \\
2211 . \\
6.094\end{array}$ \\
\hline 4400 & $\begin{array}{l}\text { y } \\
\text { H } \\
s\end{array}$ & $\begin{array}{l}58.78 \\
2070 . \\
5.829\end{array}$ & $\begin{array}{l}59.42 \\
2084 . \\
5.853\end{array}$ & $\begin{array}{l}60.07 \\
2098 . \\
5.878\end{array}$ & $\begin{array}{l}60.70 \\
2211 . \\
5.903\end{array}$ & $\begin{array}{l}61.34 \\
2125 \\
5.927\end{array}$ & $\begin{array}{l}61.98 \\
22139 . \\
5.95 i\end{array}$ & $\begin{array}{l}62.61 \\
22153 . \\
5.975\end{array}$ & $\begin{array}{l}63.24 \\
2267 . \\
5.998\end{array}$ & $\begin{array}{l}63.87 \\
2181 . \\
6.022\end{array}$ & $\begin{array}{l}64.50 \\
2195 \\
6.045\end{array}$ & $\begin{array}{l}55.13 \\
2209 . \\
6.068\end{array}$ \\
\hline 4600 & $\begin{array}{l}\nabla \\
y \\
s\end{array}$ & $\begin{array}{l}56.11 \\
20 \in \theta_{0} \\
5.803\end{array}$ & $\begin{array}{l}56.73 \\
2081 . \\
5.828\end{array}$ & $\begin{array}{l}57.34 \\
2095 . \\
5.853\end{array}$ & $\begin{array}{l}57.96 \\
22109 \\
5.877\end{array}$ & $\begin{array}{l}58.57 \\
2123 . \\
5.902\end{array}$ & $\begin{array}{l}59.18 \\
2137 . \\
5.926\end{array}$ & $\begin{array}{l}55.79 \\
2151 . \\
5.950\end{array}$ & $\begin{array}{l}60.40 \\
2165 . \\
5.974\end{array}$ & $\begin{array}{l}61.01 \\
2179 . \\
5.997\end{array}$ & $\begin{array}{l}61.61 \\
2193 \\
6.021\end{array}$ & $\begin{array}{l}62.21 \\
2207 . \\
6.040\end{array}$ \\
\hline 4800 & $\begin{array}{c}.7 \\
.4 \\
S\end{array}$ & $\begin{array}{l}53.66 \\
2065 . \\
5.779\end{array}$ & $\begin{array}{l}54.25 \\
2079 . \\
5.804\end{array}$ & $\begin{array}{l}54.85 \\
2093 . \\
5.829\end{array}$ & $\begin{array}{l}55.44 \\
22107 . \\
5.853\end{array}$ & $\begin{array}{l}56.03 \\
2121 . \\
5.878\end{array}$ & $\begin{array}{l}56.62 \\
22155 . \\
5.902\end{array}$ & $\begin{array}{l}57.21 \\
22149 . \\
5.926\end{array}$ & $\begin{array}{l}57.80 \\
21.62 . \\
5.950\end{array}$ & $\begin{array}{l}58.38 \\
2176 \\
5.973\end{array}$ & $\begin{array}{l}58.96 \\
2190 \\
5.997\end{array}$ & $\begin{array}{l}59.54 \\
2204 \\
6.020\end{array}$ \\
\hline 5000 & $\begin{array}{l}\text { D } \\
\text { H } \\
\text { S }\end{array}$ & $\begin{array}{l}51.40 \\
2063 . \\
5.755\end{array}$ & $\begin{array}{l}51.98 \\
2076 . \\
5.781\end{array}$ & $\begin{array}{l}52.55 \\
2090 . \\
5.805\end{array}$ & $\begin{array}{l}53.13 \\
2104 . \\
5.830\end{array}$ & $\begin{array}{l}53.70 \\
2118 . \\
5.855\end{array}$ & $\begin{array}{l}54.27 \\
2132 . \\
5.879\end{array}$ & $\begin{array}{l}54.83 \\
2146 . \\
5.903\end{array}$ & $\begin{array}{l}55.40 \\
2160 \\
5.927\end{array}$ & $\begin{array}{l}55.96 \\
2174 . \\
5.951\end{array}$ & $\begin{array}{l}56.52 \\
2188 \\
5.974\end{array}$ & $\begin{array}{l}57.08 \\
2202 . \\
5.997\end{array}$ \\
\hline 5200 & $\begin{array}{l}\mathbf{y} \\
\mathrm{g} \\
5\end{array}$ & $\begin{array}{l}49.32 \\
2060 . \\
5.733\end{array}$ & $\begin{array}{l}49.88 \\
2074 . \\
5.758\end{array}$ & $\begin{array}{l}50.44 \\
2088 . \\
5.783\end{array}$ & $\begin{array}{l}50.99 \\
2102 . \\
5.808\end{array}$ & $\begin{array}{l}51.54 \\
2116 . \\
5.832\end{array}$ & $\begin{array}{l}52.09 \\
2130 \\
5.857\end{array}$ & $\begin{array}{l}52.64 \\
2144 . \\
5.881\end{array}$ & $\begin{array}{l}53.18 \\
2158 . \\
5.905\end{array}$ & $\begin{array}{l}53.73 \\
2172 . \\
5.928\end{array}$ & $\begin{array}{l}54.27 \\
2186 . \\
5.952\end{array}$ & $\begin{array}{l}54.81 \\
2200 \\
5.975\end{array}$ \\
\hline$£ 400$ & $\begin{array}{l}\text { d } \\
\text { g }\end{array}$ & $\begin{array}{l}47.39 \\
2058 . \\
5.711\end{array}$ & $\begin{array}{l}47.93 \\
2072 . \\
5.736\end{array}$ & $\begin{array}{l}48.47 \\
2086 . \\
5.761\end{array}$ & $\begin{array}{l}49.01 \\
2100 . \\
5.786\end{array}$ & $\begin{array}{l}99.54 \\
2114 . \\
5.811\end{array}$ & $\begin{array}{l}50.08 \\
2128 . \\
5.835\end{array}$ & $\begin{array}{l}50.61 \\
2142 \\
5.859\end{array}$ & $\begin{array}{l}51.13 \\
2156 . \\
5.883\end{array}$ & $\begin{array}{l}51.66 \\
2170 \\
5.907\end{array}$ & $\begin{array}{l}52.19 \\
2189 . \\
5.93 i\end{array}$ & $\begin{array}{l}52.71 \\
2198 . \\
5.954\end{array}$ \\
\hline 5600 & $\begin{array}{l}\nabla \\
y \\
5\end{array}$ & $\begin{array}{l}45.60 \\
2055 . \\
-5.689\end{array}$ & $\begin{array}{l}46.13 \\
2069 . \\
5.715\end{array}$ & $\begin{array}{l}46.65 \\
2083 . \\
5.740\end{array}$ & $\begin{array}{l}47.17 \\
2097 . \\
5.765\end{array}$ & $\begin{array}{l}47.69 \\
2111 . \\
.5 .790\end{array}$ & $\begin{array}{l}48.20 \\
22125 \\
5.814\end{array}$ & $\begin{array}{l}48.72 \\
2140 . \\
5.838\end{array}$ & $\begin{array}{l}49.23 \\
22154 . \\
5.862\end{array}$ & $\begin{array}{l}49.74 \\
2168 . \\
5.886\end{array}$ & $\begin{array}{l}50.25 \\
2182 . \\
5.910\end{array}$ & $\begin{array}{l}50.76 \\
2196 . \\
5.934\end{array}$ \\
\hline 5800 & $\begin{array}{l}\text { D } \\
\text { a } \\
\text { s }\end{array}$ & $\begin{array}{l}43.94 \\
20 \leq 3 . \\
5.669\end{array}$ & $\begin{array}{l}44.45 \\
2067 . \\
5.699\end{array}$ & 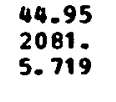 & $\begin{array}{l}45.46 \\
2095 . \\
5.744\end{array}$ & 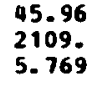 & $\begin{array}{l}96.46 \\
22123 . \\
5.796\end{array}$ & $\begin{array}{l}46.96 . \\
22.177 . \\
5.818\end{array}$ & $\begin{array}{l}47.46 \\
2151 . \\
5.892\end{array}$ & $\begin{array}{l}47.95 \\
2166 . \\
5.866\end{array}$ & $\begin{array}{l}48.45 \\
2180 . \\
5.890\end{array}$ & $\begin{array}{l}48.94 \\
2194 . \\
5.914\end{array}$ \\
\hline 6000 & $\begin{array}{l}V \\
H \\
S\end{array}$ & $\begin{array}{l}42.38 \\
20 \leq 0 . \\
5.649\end{array}$ & $\begin{array}{l}42.88 \\
2064 \\
5.674\end{array}$ & $\begin{array}{l}43.37 \\
2078 . \\
5.699\end{array}$ & $\begin{array}{l}43.86 \\
2093 . \\
5.729\end{array}$ & $\begin{array}{l}44.35 \\
2107 \\
5.749\end{array}$ & $\begin{array}{l}44.84 \\
21214 \\
5.774\end{array}$ & $\begin{array}{l}45.32 \\
2135 . \\
5.798\end{array}$ & $\begin{array}{l}45.80 \\
2149 . \\
5.823\end{array}$ & $\begin{array}{l}46.29 \\
2163 \\
5.847\end{array}$ & $\begin{array}{l}46.77 \\
2178 . \\
5.870\end{array}$ & $\begin{array}{l}47.24 \\
2192 . \\
5.899\end{array}$ \\
\hline
\end{tabular}


Table 10 (Continued)

Temperature range: 280 to $330^{\circ} \mathrm{C}$

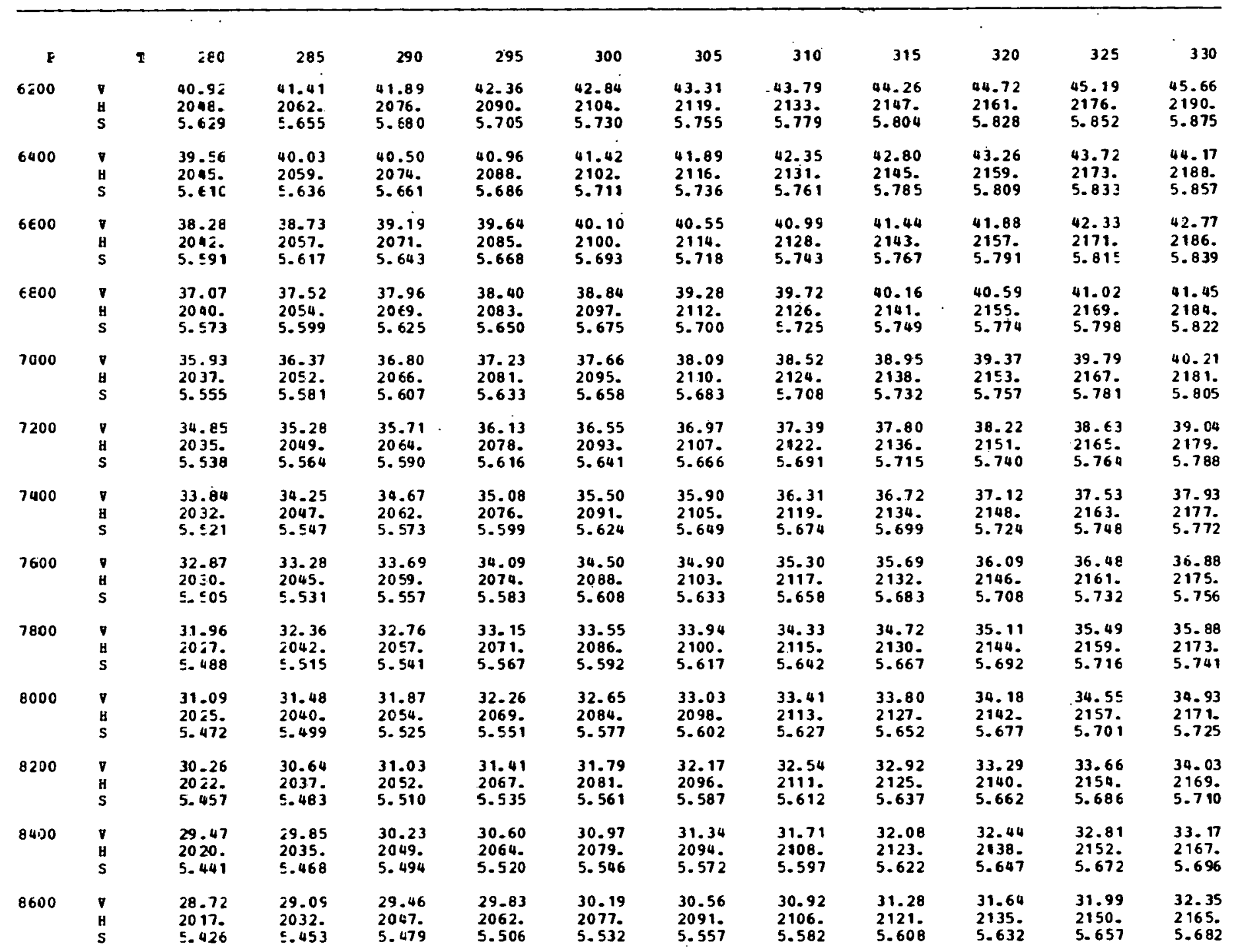


Table 10 (Continued)

Temperature range: 280 to $330^{\circ} \mathrm{C}$

\begin{tabular}{|c|c|c|c|c|c|c|c|c|c|c|c|c|}
\hline P & I & ¿80 & 285 & 290 & 295 & 300 & 305 & 310 & 315 & 320 & 325 & 330 \\
\hline 8800 & $\begin{array}{l}y \\
y \\
s\end{array}$ & $\begin{array}{l}28.00 \\
2015 \\
5.411\end{array}$ & $\begin{array}{l}28.37 \\
2030 . \\
5.438\end{array}$ & $\begin{array}{l}28.73 \\
2045 . \\
5.465\end{array}$ & $\begin{array}{l}29.09 \\
2059 . \\
5.491\end{array}$ & $\begin{array}{l}29.45 \\
2074 \\
5.517\end{array}$ & $\begin{array}{l}29.81 \\
2089 \\
5.543\end{array}$ & $\begin{array}{l}30.16 \\
2104 . \\
5.568\end{array}$ & $\begin{array}{l}30.52 \\
2119 . \\
5.593\end{array}$ & $\begin{array}{l}30.87 \\
2133 . \\
5.618\end{array}$ & $\begin{array}{l}31.22 \\
2148 . \\
5.643\end{array}$ & $\begin{array}{l}31.57 \\
2163 . \\
5.668\end{array}$ \\
\hline 9000 & $\begin{array}{l}\text { D } \\
\text { H } \\
\text { s }\end{array}$ & $\begin{array}{l}27.31 \\
2012 \\
5.397\end{array}$ & $\begin{array}{l}27.67 \\
2027 \\
5.424\end{array}$ & $\begin{array}{l}28.03 \\
2042 . \\
5.450\end{array}$ & $\begin{array}{l}28.39 \\
2057 \\
5.477\end{array}$ & $\begin{array}{l}28.74 \\
2072 . \\
5.503\end{array}$ & $\begin{array}{l}29.09 \\
2087 \\
5.529\end{array}$ & $\begin{array}{l}29.44 \\
2102 . \\
5.554\end{array}$ & $\begin{array}{l}29.79 \\
2116 . \\
5.579\end{array}$ & $\begin{array}{l}30.13 \\
2131 . \\
5.604\end{array}$ & $\begin{array}{l}30.4 E \\
2146 . \\
5.629\end{array}$ & $\begin{array}{l}30.82 \\
2161 . \\
5.654\end{array}$ \\
\hline 9200 & $\begin{array}{l}\text { D } \\
\text { us }\end{array}$ & $\begin{array}{l}26.66 \\
2010 . \\
5.383\end{array}$ & $\begin{array}{l}27.01 \\
2025 . \\
5.410\end{array}$ & $\begin{array}{l}27.36 \\
2040 . \\
5.436\end{array}$ & $\begin{array}{l}27.71 \\
2055 . \\
5.463\end{array}$ & $\begin{array}{l}28.06 \\
2070 . \\
5.489\end{array}$ & $\begin{array}{l}28.40 \\
2085 . \\
5.515\end{array}$ & $\begin{array}{l}28.75 \\
2099 . \\
5.540\end{array}$ & $\begin{array}{l}29.09 \\
2114 . \\
5.566\end{array}$ & $\begin{array}{l}29.43 \\
2129 . \\
5.591\end{array}$ & $\begin{array}{l}29.77 \\
29.44 . \\
5.616\end{array}$ & $\begin{array}{l}30.10 \\
2159 . \\
5.640\end{array}$ \\
\hline 9400 & $\begin{array}{l}\mathrm{D} \\
\mathrm{H} \\
\mathrm{S}\end{array}$ & $\begin{array}{l}26.03 \\
2007 . \\
5.36 \mathrm{E}\end{array}$ & $\begin{array}{l}26.38 \\
2022 . \\
5.396\end{array}$ & $\begin{array}{l}26.72 \\
2037 . \\
5.422\end{array}$ & $\begin{array}{l}27.07 \\
2052 . \\
5.409\end{array}$ & $\begin{array}{l}27.41 \\
2067 . \\
5.475\end{array}$ & $\begin{array}{l}27.75 \\
2082 \\
5.501\end{array}$ & $\begin{array}{l}28.09 \\
2097 \\
5.527\end{array}$ & $\begin{array}{l}28.42 \\
2112 . \\
5.552\end{array}$ & $\begin{array}{l}28.75 \\
2127 \\
5.577\end{array}$ & $\begin{array}{l}29.09 \\
2142 . \\
5.602\end{array}$ & $\begin{array}{l}29.42 \\
2157 \\
5.627\end{array}$ \\
\hline 9600 & $\begin{array}{l}\nabla \\
\mathbf{y} \\
\mathrm{s}\end{array}$ & $\begin{array}{l}25.43 \\
2005 . \\
5.355\end{array}$ & $\begin{array}{l}25.77 \\
2020 . \\
5.382\end{array}$ & $\begin{array}{l}26.11 \\
2035 . \\
5.409\end{array}$ & $\begin{array}{l}26.45 \\
2050 . \\
5.435\end{array}$ & $\begin{array}{l}26.78 \\
2065 . \\
5.462\end{array}$ & $\begin{array}{l}27.12 \\
2080 . \\
5.488 .\end{array}$ & $\begin{array}{l}27.45 \\
2095 . \\
5.513\end{array}$ & $\begin{array}{l}27.78 \\
2110 . \\
5.539\end{array}$ & $\begin{array}{l}28.11 \\
2125 . \\
5.564\end{array}$ & $\begin{array}{l}28.44 \\
2140 . \\
5.589\end{array}$ & $\begin{array}{l}28.76 \\
2154 . \\
5.614\end{array}$ \\
\hline 9800 & $\begin{array}{l}\mathrm{D} \\
\mathrm{H} \\
\mathrm{S}\end{array}$ & $\begin{array}{l}24.85 \\
2002 . \\
5.341\end{array}$ & $\begin{array}{l}25.19 \\
2017 . \\
5.368\end{array}$ & $\begin{array}{l}25.52 \\
2033 . \\
5.395\end{array}$ & $\begin{array}{l}25.86 \\
2048 . \\
5.422\end{array}$ & $\begin{array}{l}26.19 \\
2063 . \\
5.448\end{array}$ & $\begin{array}{l}26.51 \\
2078 \\
5.474\end{array}$ & $\begin{array}{l}26.84 \\
2093 . \\
5.500\end{array}$ & $\begin{array}{l}27.17 \\
2108 . \\
5.526\end{array}$ & $\begin{array}{l}27.49 \\
2123 . \\
5.551\end{array}$ & $\begin{array}{l}27.81 \\
2138 \\
5.576\end{array}$ & $\begin{array}{l}28.13 \\
2152 . \\
5.600\end{array}$ \\
\hline 10000 & $\begin{array}{l}\text { D } \\
\mathrm{u} \\
\mathrm{s}\end{array}$ & $\begin{array}{l}24.29 \\
2000 . \\
5.328\end{array}$ & $\begin{array}{l}24.63 \\
20.5 . \\
\vdots .355\end{array}$ & $\begin{array}{l}24.96 \\
2030 . \\
5.382\end{array}$ & $\begin{array}{l}25.29 \\
2045 . \\
5.409\end{array}$ & $\begin{array}{l}25.61 \\
2060 . \\
5.435\end{array}$ & $\begin{array}{l}.25 .93 \\
2075 \\
5.461\end{array}$ & $\begin{array}{l}26.26 \\
2590 . \\
5-487\end{array}$ & $\begin{array}{l}26.58 \\
2105 . \\
5.513\end{array}$ & $\begin{array}{l}26.89 \\
2120 . \\
5.538\end{array}$ & $\begin{array}{l}27.21 \\
2135 \\
5.563\end{array}$ & $\begin{array}{l}27.53 \\
2150 \\
5.588\end{array}$ \\
\hline 10100 & $\begin{array}{l}\text { D } \\
\text { H } \\
\text { s }\end{array}$ & $\begin{array}{l}24.02 \\
1998 . \\
5.321\end{array}$ & $\begin{array}{l}24.36 \\
20.34 \\
5.348\end{array}$ & $\begin{array}{l}24.68 \\
2029 . \\
5.375\end{array}$ & $\begin{array}{l}25.01 \\
2044 \\
5.402\end{array}$ & $\begin{array}{l}25.33 \\
2059 . \\
5.429\end{array}$ & $\begin{array}{l}25.65 \\
2074 \\
5.455\end{array}$ & $\begin{array}{l}25.97 \\
2089 \\
5.481\end{array}$ & $\begin{array}{l}26.29 \\
2104 \\
5.507\end{array}$ & $\begin{array}{l}26.61 \\
219 . \\
5.532\end{array}$ & $\begin{array}{l}26.92 \\
2134 \\
5.557\end{array}$ & $\begin{array}{l}27.23 \\
2149 . \\
5.582\end{array}$ \\
\hline 10200 & $\begin{array}{l}\nabla \\
\text { H } \\
\text { S }\end{array}$ & $\begin{array}{l}23.76 \\
1997 . \\
5.314\end{array}$ & $\begin{array}{l}24.09 \\
20.12 \\
5.342\end{array}$ & $\begin{array}{l}24.41 \\
2028 \\
5.369\end{array}$ & $\begin{array}{l}24.74 \\
2043 . \\
5.396\end{array}$ & $\begin{array}{l}25.06 \\
2058 . \\
5.422\end{array}$ & $\begin{array}{l}25.38 \\
2073 . \\
5.449\end{array}$ & $\begin{array}{l}25.65 \\
2008 . \\
5.475\end{array}$ & $\begin{array}{l}26.01 \\
2103 \\
5.500\end{array}$ & $\begin{array}{l}26.32 \\
2118 . \\
5.526\end{array}$ & $\begin{array}{l}26.63 \\
2133 \\
5.551\end{array}$ & $\begin{array}{l}26.94 \\
2149 . \\
5.576\end{array}$ \\
\hline 10300 & $\begin{array}{l}\text { V } \\
\text { H } \\
\text { s }\end{array}$ & $\begin{array}{l}23.50 \\
1996 . \\
5.308\end{array}$ & $\begin{array}{l}23.83 \\
2011 \\
\text { s.335. }\end{array}$ & $\begin{array}{l}24.15 \\
20.26 . \\
5.363\end{array}$ & $\begin{array}{l}24.47 \\
20.42 \\
5.389\end{array}$ & $\begin{array}{l}24.79 \\
2057 . \\
5.016\end{array}$ & $\begin{array}{l}25.11 \\
2072 . \\
5.442\end{array}$ & $\begin{array}{l}25.42 \\
2087 . \\
5.468\end{array}$ & $\begin{array}{l}25.73 \\
2102 . \\
5.494\end{array}$ & $\begin{array}{l}26.09 \\
2117 . \\
5.520\end{array}$ & $\begin{array}{l}26.35 \\
2132 . \\
5.545\end{array}$ & $\begin{array}{l}26.66 \\
2147 . \\
5.570\end{array}$ \\
\hline 10400 & $\begin{array}{l}\nabla \\
\text { H } \\
S\end{array}$ & $\begin{array}{l}23.25 \\
1995 . \\
5.301\end{array}$ & $\begin{array}{l}23.57 \\
2010 . \\
5.329\end{array}$ & $\begin{array}{l}23.89 \\
2025 . \\
5.356\end{array}$ & $\begin{array}{l}24.21 \\
2041 \\
5.383\end{array}$ & $\begin{array}{l}24.53 \\
2056 . \\
5.910\end{array}$ & $\begin{array}{l}24.84 \\
2071 . \\
5.436\end{array}$ & $\begin{array}{l}25.15 \\
2086 . \\
5.462\end{array}$ & $\begin{array}{l}25.46 \\
2101 . \\
5.488\end{array}$ & $\begin{array}{l}25.77 \\
2116 . \\
5.513\end{array}$ & $\begin{array}{l}26.08 \\
2131 . \\
5.539\end{array}$ & $\begin{array}{l}26.38 \\
2146 . \\
5.564\end{array}$ \\
\hline $10 \leq 00$ & $\begin{array}{l}\mathrm{I} \\
\mathrm{B} \\
\mathrm{S}\end{array}$ & $\begin{array}{l}23.00 \\
1993 . \\
5.295\end{array}$ & $\begin{array}{l}23.32 \\
2009 . \\
5.322\end{array}$ & $\begin{array}{l}23.64 \\
2024 . \\
5.350\end{array}$ & $\begin{array}{l}23.95 \\
2039 \\
5.377\end{array}$ & $\begin{array}{l}24.27 \\
2055 . \\
5.403\end{array}$ & $\begin{array}{l}24.58 \\
2070 . \\
5.430\end{array}$ & $\begin{array}{l}24.89 \\
2085 . \\
5.456\end{array}$ & $\begin{array}{l}25.20 \\
2100 . \\
5.482\end{array}$ & $\begin{array}{l}25.51 \\
2115 \\
5.507\end{array}$ & $\begin{array}{l}25.81 \\
2130 . \\
5.532\end{array}$ & $\begin{array}{l}26.11 \\
2145 . \\
5.558\end{array}$ \\
\hline 10600 & $\begin{array}{l}\mathrm{V} \\
\mathrm{H} \\
\mathrm{s}\end{array}$ & $\begin{array}{l}22.75 \\
1992 . \\
5.288\end{array}$ & $\begin{array}{l}23.07 \\
2007 . \\
5.316\end{array}$ & $\begin{array}{l}23.39 \\
2023 . \\
5.303\end{array}$ & $\begin{array}{l}23.70 \\
2038 . \\
5.370\end{array}$ & $\begin{array}{l}24.02 \\
2053 . \\
5.397\end{array}$ & $\begin{array}{l}24.33 \\
2069 \\
5.424\end{array}$ & $\begin{array}{l}24.63 \\
2084 . \\
5.450\end{array}$ & $\begin{array}{l}24.94 \\
2099 . \\
5.076\end{array}$ & $\begin{array}{l}25.24 \\
2114 \\
5.501\end{array}$ & $\begin{array}{l}25.55 \\
2129 . \\
5.526\end{array}$ & $\begin{array}{l}25.85 \\
2144 . \\
5.551\end{array}$ \\
\hline
\end{tabular}


Table 10 (Continued)

Temperature range: 280 to $330^{\circ} \mathrm{C}$

\begin{tabular}{|c|c|c|c|c|c|c|c|c|c|c|c|c|}
\hline $\mathbf{p}$ & $I$ & 280 & 285 & 290 & 295 & 300 & 305 & $310^{\circ}$ & 315 & 320 & 325 & 330 \\
\hline 10700 & : & $\begin{array}{l}22.51 \\
1951 \\
5.282\end{array}$ & $\begin{array}{l}22.83 \\
2006 . \\
5.310\end{array}$ & $\begin{array}{l}23.15 \\
2022 . \\
5.337\end{array}$ & $\begin{array}{l}23.46 \\
2037 . \\
5.364\end{array}$ & $\begin{array}{l}23.77 \\
2052 . \\
5.391\end{array}$ & $\begin{array}{l}24.07 \\
2067 \\
5.417\end{array}$ & $\begin{array}{l}24.38 \\
2083 . \\
5.444\end{array}$ & $\begin{array}{l}24.68 \\
2098 . \\
5.469\end{array}$ & $\begin{array}{l}24.99 \\
2113: \\
\text { !. } 495\end{array}$ & $\begin{array}{l}25.29 \\
2128 . \\
5.520\end{array}$ & $\begin{array}{l}25.58 \\
2143 . \\
5.546\end{array}$ \\
\hline 10800 & $\begin{array}{l}y \\
y \\
s\end{array}$ & $\begin{array}{l}22.28 \\
1990 \\
5.576\end{array}$ & $\begin{array}{l}22.59 \\
2005 \\
5.303\end{array}$ & $\begin{array}{l}22.91 \\
22020 . \\
5.331\end{array}$ & $\begin{array}{l}23.22 \\
2036 . \\
5.358\end{array}$ & $\begin{array}{l}23.52 \\
2051 . \\
5.385\end{array}$ & $\begin{array}{l}23.83 \\
2066 \\
5.411\end{array}$ & $\begin{array}{l}24.13 \\
2082 . \\
5.438\end{array}$ & $\begin{array}{l}24.43 \\
2097 . \\
5.463\end{array}$ & $\begin{array}{l}24.73 \\
2112 . \\
5.489\end{array}$ & $\begin{array}{l}25.03 \\
2127 . \\
5.514\end{array}$ & $\begin{array}{l}25.33 \\
2142 . \\
5.540\end{array}$ \\
\hline 10900 & $\begin{array}{l}\text { y } \\
\text { s } \\
\text { s }\end{array}$ & $\begin{array}{l}22.05 \\
19 \varepsilon 8 . \\
5.269\end{array}$ & $\begin{array}{l}22.36 \\
2004 . \\
5.297\end{array}$ & $\begin{array}{l}22.67 \\
20.9 . \\
5.325\end{array}$ & $\begin{array}{l}22.98 \\
2035 . \\
5.352\end{array}$ & $\begin{array}{l}23.28 \\
2050 . \\
5.379\end{array}$ & $\begin{array}{l}23.59 \\
2065 . \\
5.405\end{array}$ & $\begin{array}{l}23.89 \\
2080 . \\
5.431\end{array}$ & $\begin{array}{l}24.19 \\
2096 . \\
5.457\end{array}$ & $\begin{array}{l}24.49 \\
2111 . \\
5.483\end{array}$ & $\begin{array}{l}24.78 \\
2126 . \\
5.509\end{array}$ & $\begin{array}{l}25.08 \\
2141 . \\
5.530\end{array}$ \\
\hline 11000 & $\begin{array}{l}y \\
y \\
\text { s }\end{array}$ & $\begin{array}{l}21.82 \\
19 \varepsilon 7 \\
5.263\end{array}$ & $\begin{array}{l}22.13 \\
2003 . \\
\Xi .291\end{array}$ & $\begin{array}{l}22.44 \\
20.18 \\
5.319\end{array}$ & $\begin{array}{l}22.74 \\
20.73 \\
5.346\end{array}$ & $\begin{array}{l}23.05 \\
2049 . \\
5.373\end{array}$ & $\begin{array}{l}23.35 \\
2064 . \\
5.399\end{array}$ & $\begin{array}{l}23.65 \\
2079 . \\
\mathrm{s}-426\end{array}$ & $\begin{array}{l}23.95 \\
2095 . \\
5.45 i\end{array}$ & $\begin{array}{l}24.24 \\
2110 . \\
5.477\end{array}$ & $\begin{array}{l}24.54 \\
2125 . \\
5.503\end{array}$ & $\begin{array}{l}24.83 \\
2140 . \\
5.528\end{array}$ \\
\hline 11100 & $\begin{array}{l}\text { I } \\
\text { u } \\
\text { s }\end{array}$ & $\begin{array}{l}21.60 \\
19 \varepsilon 6 . \\
5.257\end{array}$ & $\begin{array}{l}21.91 \\
2001 . \\
5.285\end{array}$ & $\begin{array}{l}22.21 \\
2017 . \\
5.312\end{array}$ & $\begin{array}{l}22.52 \\
22032 . \\
5.340\end{array}$ & $\begin{array}{l}22.82 \\
2048 . \\
5.367\end{array}$ & $\begin{array}{l}23.12 \\
2063 . \\
5.393\end{array}$ & $\begin{array}{l}23.41 \\
2078 . \\
5.420\end{array}$ & $\begin{array}{l}23.71 \\
2093 . \\
5.446\end{array}$ & $\begin{array}{l}24.00 \\
2109 \\
5.471\end{array}$ & $\begin{array}{l}24.29 \\
2124 \\
5.497\end{array}$ & $\begin{array}{l}24.59 \\
2139 . \\
5.522\end{array}$ \\
\hline 11200 & $\begin{array}{l}\nabla \\
\stackrel{u}{s} \\
s\end{array}$ & $\begin{array}{l}21-38 \\
19 E 4 . \\
5.251\end{array}$ & $\begin{array}{l}21.68 \\
2000 . \\
5.279\end{array}$ & $\begin{array}{l}21.99 \\
20.96 \\
5.306\end{array}$ & $\begin{array}{l}22.29 \\
2231 . \\
5.334\end{array}$ & $\begin{array}{l}22.59 \\
2046 . \\
5.361\end{array}$ & $\begin{array}{l}22.89 \\
2062 . \\
5.387\end{array}$ & $\begin{array}{l}23.18 \\
2077 \\
5.414\end{array}$ & $\begin{array}{l}23.48 \\
2092 . \\
5.440\end{array}$ & $\begin{array}{l}23.77 \\
22108 . \\
5.465\end{array}$ & $\begin{array}{l}24.06 \\
2123 \\
5.491\end{array}$ & $\begin{array}{l}24.35 \\
2138 . \\
5.516\end{array}$ \\
\hline 11300 & $\begin{array}{l}\nabla \\
\text { u } \\
s\end{array}$ & $\begin{array}{l}21.16 \\
.983 . \\
5.244\end{array}$ & $\begin{array}{l}21.47 \\
1999 \\
5.273\end{array}$ & $\begin{array}{l}21.77 \\
20.4 . \\
5.300\end{array}$ & $\begin{array}{l}22.07 \\
2000 . \\
5.328\end{array}$ & $\begin{array}{l}22.37 \\
2245 . \\
5.355\end{array}$ & $\begin{array}{l}22.66 \\
2061 \\
5.381\end{array}$ & $\begin{array}{l}22.96 \\
2076 . \\
5,408\end{array}$ & $\begin{array}{l}23.25 \\
2301 . \\
5.434\end{array}$ & $\begin{array}{l}23.54 \\
2106 . \\
5.460\end{array}$ & $\begin{array}{l}23.83 \\
2122 . \\
5.485\end{array}$ & $\begin{array}{l}24.11 \\
2137 . \\
5.511\end{array}$ \\
\hline $114: 30$ & $\begin{array}{l}\text { I } \\
\text { B } \\
\text { s }\end{array}$ & $\begin{array}{l}20.95 \\
1982 . \\
5.238\end{array}$ & $\begin{array}{l}21.25 \\
1998 . \\
5.266\end{array}$ & $\begin{array}{l}21.55 \\
2013 . \\
5.294\end{array}$ & $\begin{array}{l}21.85 \\
2009 . \\
5 .: 22\end{array}$ & $\begin{array}{l}22.15 \\
22044 . \\
5.349\end{array}$ & $\begin{array}{l}22.44 \\
2059 \\
5.376\end{array}$ & $\begin{array}{l}22.73 \\
22075 . \\
5.402\end{array}$ & $\begin{array}{l}23.02 \\
2000 \\
5.428\end{array}$ & $\begin{array}{l}23.31 \\
2105 . \\
5.454\end{array}$ & $\begin{array}{l}23.60 \\
2121 . \\
5.480\end{array}$ & $\begin{array}{l}25.88 \\
2136 . \\
5.505\end{array}$ \\
\hline $115: 00$ & $\begin{array}{l}\text { v } \\
\text { u } \\
\text { s }\end{array}$ & $\begin{array}{l}20.74 \\
19 \mathrm{g1}^{2} \\
5.232\end{array}$ & $\begin{array}{l}21.04 \\
1996 . \\
5.260\end{array}$ & $\begin{array}{l}21.34 \\
2012 . \\
5.288\end{array}$ & $\begin{array}{l}21.64 \\
20.7 . \\
5.316\end{array}$ & $\begin{array}{l}21.93 \\
2043 . \\
5.343\end{array}$ & $\begin{array}{l}22.22 \\
2058 . \\
5.370\end{array}$ & $\begin{array}{l}22.51 \\
22074 \\
5.396\end{array}$ & $\begin{array}{l}22.80 \\
2089 . \\
5.422\end{array}$ & $\begin{array}{l}23.09 \\
2104 . \\
5.448\end{array}$ & $\begin{array}{l}23.37 \\
2120 . \\
5.474\end{array}$ & $\begin{array}{l}23.65 \\
2135 . \\
5.499\end{array}$ \\
\hline 116,30 & $\begin{array}{l}y \\
y \\
s\end{array}$ & $\begin{array}{l}20.54 \\
1979 . \\
5.226\end{array}$ & $\begin{array}{l}20.84 \\
1995 . \\
5.255\end{array}$ & $\begin{array}{l}21.14 \\
2011 . \\
5.282\end{array}$ & $\begin{array}{l}21.43 \\
2006 . \\
5 .=10\end{array}$ & $\begin{array}{l}21.72 \\
2042 . \\
5.337\end{array}$ & $\begin{array}{l}22.01 \\
2057 \\
5.364\end{array}$ & $\begin{array}{l}22.30 \\
2203 . \\
5.390\end{array}$ & $\begin{array}{l}22.59 \\
2088 \\
5.417\end{array}$ & $\begin{array}{l}22.87 \\
2103 . \\
5.443\end{array}$ & $\begin{array}{l}23.15 \\
2119 . \\
5.468\end{array}$ & $\begin{array}{l}23.43 \\
2134 . \\
5.490\end{array}$ \\
\hline 11730 & $\begin{array}{l}\mathrm{D} \\
\mathrm{B} \\
\mathrm{s}\end{array}$ & $\begin{array}{l}20.34 \\
1978 . \\
5.220\end{array}$ & $\begin{array}{l}20.64 \\
1994 . \\
5-249\end{array}$ & $\begin{array}{l}20.93 \\
2009 . \\
5.276\end{array}$ & $\begin{array}{l}21.222 \\
2005 . \\
5.304\end{array}$ & $\begin{array}{l}21.51 \\
2041 \\
5.331\end{array}$ & $\begin{array}{l}21.80 \\
2056 . \\
5.358\end{array}$ & $\begin{array}{l}22.09 \\
2072 . \\
5.385\end{array}$ & $\begin{array}{l}22.37 \\
2087 \\
5.411\end{array}$ & $\begin{array}{l}22.65 \\
2102 . \\
5.437\end{array}$ & $\begin{array}{l}22.93 \\
2118 . \\
5.463\end{array}$ & $\begin{array}{l}23.21 \\
2133 . \\
5.488\end{array}$ \\
\hline 11800 & $\begin{array}{l}\text { V } \\
\text { y } \\
s\end{array}$ & $\begin{array}{l}20.14 \\
1977 \\
5.234\end{array}$ & $\begin{array}{l}20.44 \\
1993 \\
5.203\end{array}$ & $\begin{array}{l}20.73 \\
2008 \\
5.271\end{array}$ & $\begin{array}{l}21.02 \\
2024 . \\
5.298\end{array}$ & $\begin{array}{l}21.31 \\
2039 . \\
5.325\end{array}$ & $\begin{array}{l}21.60 \\
2055 . \\
5.352\end{array}$ & $\begin{array}{l}21.88 \\
2070 . \\
5.379\end{array}$ & $\begin{array}{l}22.16 \\
2086 \\
5.405\end{array}$ & $\begin{array}{l}22.44 \\
2101 . \\
5.431\end{array}$ & $\begin{array}{l}22.72 \\
2116 . \\
5.457\end{array}$ & $\begin{array}{l}23.00 \\
2132 . \\
5.482\end{array}$ \\
\hline 11900 & $\begin{array}{l}\text { J } \\
\text { u } \\
\text { s }\end{array}$ & $\begin{array}{l}19.95 \\
1976 . \\
5.208\end{array}$ & $\begin{array}{l}20.24 \\
1991 \\
5.237\end{array}$ & $\begin{array}{l}20-53 \\
2007 \\
5.265\end{array}$ & $\begin{array}{l}20.82 \\
2033 \\
5.392\end{array}$ & $\begin{array}{l}21.11 \\
2038 . \\
5.320\end{array}$ & $\begin{array}{l}21.39 \\
2054 . \\
5.347\end{array}$ & $\begin{array}{l}21.68 \\
20.69 \\
5.373\end{array}$ & $\begin{array}{l}21.96 \\
2085 \\
5.400\end{array}$ & $\begin{array}{l}22.23 \\
21100 \\
5.426\end{array}$ & $\begin{array}{l}22.51 \\
215 \\
5.45 i\end{array}$ & $\begin{array}{l}22.79 \\
2131 \\
5.47\end{array}$ \\
\hline
\end{tabular}


Table 10 (Continued)

Temperature range: 280 to $330^{\circ} \mathrm{C}$

\begin{tabular}{|c|c|c|c|c|c|c|c|c|c|c|c|c|}
\hline $\mathbf{P}$ & $x$ & 280 & 285 & 290 & 295 & 300 & 305 & 310 & 315 & 320 & 325 & 330 \\
\hline 12000 & $\begin{array}{l}\text { y } \\
\text { a } \\
\text { s }\end{array}$ & $\begin{array}{l}19.76 \\
1974 \\
5.202\end{array}$ & $\begin{array}{l}20.05 \\
\mathbf{9 9 9 0} \\
5.231\end{array}$ & $\begin{array}{l}20.34 \\
2006 . \\
5.259\end{array}$ & $\begin{array}{l}20.63 \\
2022 . \\
5.287\end{array}$ & $\begin{array}{l}20.91 \\
2037 . \\
5.314\end{array}$ & $\begin{array}{l}21.19 \\
2053 . \\
5.341\end{array}$ & $\begin{array}{l}21.47 \\
2068 . \\
5.368\end{array}$ & $\begin{array}{l}21.75 \\
2084 \\
5.394\end{array}$ & $\begin{array}{l}22.03 \\
2099 . \\
5.420\end{array}$ & $\begin{array}{l}22.30 \\
214 . \\
5.946\end{array}$ & $\begin{array}{l}22.58 \\
2130 \\
5.471\end{array}$ \\
\hline 12200 & $\begin{array}{l}y \\
\text { g } \\
\text { s }\end{array}$ & $\begin{array}{l}19.38 \\
1972 . \\
5.191\end{array}$ & $\begin{array}{l}19.67 \\
1988 . \\
5.219\end{array}$ & $\begin{array}{l}19.96 \\
2003 . \\
5.248\end{array}$ & $\begin{array}{l}20.24 \\
2019 . \\
5.275\end{array}$ & $\begin{array}{l}20.52 \\
2035 . \\
5.303\end{array}$ & $\begin{array}{l}20.80 \\
2050 . \\
5.330\end{array}$ & $\begin{array}{l}21.08 \\
2066 \\
5.357\end{array}$ & $\begin{array}{l}21.36 \\
2081 . \\
5.383\end{array}$ & $\begin{array}{l}21.63 \\
2097 \\
5.409\end{array}$ & $\begin{array}{l}21.90 \\
2112 . \\
5.435\end{array}$ & $\begin{array}{l}22.17 \\
2128 . \\
5.461\end{array}$ \\
\hline 12400 & $\begin{array}{l}8 \\
8 \\
5\end{array}$ & $\begin{array}{l}19.02 \\
1969 . \\
5.179\end{array}$ & $\begin{array}{l}19.31 \\
1985 . \\
5.208\end{array}$ & $\begin{array}{l}19.59 \\
2001 . \\
5.236\end{array}$ & $\begin{array}{l}19.87 \\
2017 . \\
5.264\end{array}$ & $\begin{array}{l}20.15 \\
2032 . \\
5.292\end{array}$ & $\begin{array}{l}20.43 \\
2048 . \\
5.319\end{array}$ & $\begin{array}{l}20.70 \\
2064 . \\
5.346\end{array}$ & $\begin{array}{l}20.97 \\
2079 . \\
5.372\end{array}$ & $\begin{array}{l}21.24 \\
2095 . \\
5.398\end{array}$ & $\begin{array}{l}21.51 \\
2110 . \\
5.424\end{array}$ & $\begin{array}{l}21.78 \\
2126 . \\
5.450\end{array}$ \\
\hline 12600 & $\begin{array}{l}8 \\
\mathrm{y} \\
\mathrm{s}\end{array}$ & $\begin{array}{l}18.67 \\
1967 . \\
5.168\end{array}$ & $\begin{array}{l}18.96 \\
1983 \\
5.197\end{array}$ & $\begin{array}{l}19.24 \\
1999 . \\
5.225\end{array}$ & $\begin{array}{l}19.51 \\
2014 . \\
5.253\end{array}$ & $\begin{array}{l}19.79 \\
2030 . \\
5.281\end{array}$ & $\begin{array}{l}20.06 \\
2046- \\
5.308\end{array}$ & $\begin{array}{l}20.33 \\
2061 . \\
5.335\end{array}$ & $\begin{array}{l}20.60 \\
2077 \\
5.361\end{array}$ & $\begin{array}{l}20.87 \\
2093 . \\
5.388\end{array}$ & $\begin{array}{l}21.14 \\
2108- \\
5.414\end{array}$ & $\begin{array}{l}21.40 \\
2123 \\
5.439\end{array}$ \\
\hline 12800 & $\begin{array}{l}V \\
\text { B } \\
s\end{array}$ & $\begin{array}{l}18.34 \\
8964 . \\
5.157\end{array}$ & $\begin{array}{l}18.62 \\
1980 . \\
\leq .185\end{array}$ & $\begin{array}{l}18.89 \\
1996 . \\
5.214\end{array}$ & $\begin{array}{l}19.17 \\
2012 . \\
5.242\end{array}$ & $\begin{array}{l}19.44 \\
2028 . \\
5.270\end{array}$ & $\begin{array}{l}19.71 \\
2044 . \\
5.297\end{array}$ & $\begin{array}{l}19.98 \\
2059 . \\
5.324\end{array}$ & $\begin{array}{l}20.24 \\
2075 \\
5.35 i\end{array}$ & $\begin{array}{l}20.51 \\
2090 . \\
5.377\end{array}$ & $\begin{array}{l}20.77 \\
2106 . \\
5.403\end{array}$ & $\begin{array}{l}21.03 \\
2121 \\
5.429\end{array}$ \\
\hline 13000 & $\begin{array}{l}7 \\
\text { i } \\
s\end{array}$ & $\begin{array}{l}18.01 \\
1962 . \\
5.145\end{array}$ & $\begin{array}{l}18.28 \\
1978 \\
5.174\end{array}$ & $\begin{array}{l}18.56 \\
1994 . \\
5.203\end{array}$ & $\begin{array}{l}18.83 \\
20010 . \\
5.231\end{array}$ & $\begin{array}{l}19.10 \\
2025 . \\
5.259\end{array}$ & $\begin{array}{l}19.37 \\
2041 . \\
5.286\end{array}$ & $\begin{array}{l}19.63 \\
2057 \\
5.313\end{array}$ & $\begin{array}{l}19.89 \\
2073 . \\
5.340\end{array}$ & $\begin{array}{l}20.16 \\
2088 . \\
5.367\end{array}$ & $\begin{array}{l}20.92 \\
2104 . \\
5.393\end{array}$ & $\begin{array}{l}20.67 \\
2119 . \\
5.419\end{array}$ \\
\hline 13200 & $\begin{array}{l}7 \\
\text { g } \\
5\end{array}$ & $\begin{array}{l}17.69 \\
\mathbf{1 9} 59 . \\
5.134\end{array}$ & $\begin{array}{l}17.96 \\
1975 \\
5: 163\end{array}$ & $\begin{array}{l}18.24 \\
1991 . \\
5.192\end{array}$ & $\begin{array}{l}18.50 \\
2007 . \\
5.220\end{array}$ & $\begin{array}{l}18.77 \\
2023 . \\
5.248\end{array}$ & $\begin{array}{l}19.03 \\
2039 . \\
5.276\end{array}$ & $\begin{array}{l}19.30 \\
2055 . \\
5.303\end{array}$ & $\begin{array}{l}19.56 \\
2070 . \\
5.330\end{array}$ & $\begin{array}{l}19.82 \\
2086 . \\
5.356\end{array}$ & $\begin{array}{l}20.07 \\
2102 . \\
5.382\end{array}$ & $\begin{array}{l}20.33 \\
2117 \\
5.408\end{array}$ \\
\hline 13400 & $\begin{array}{l}\text { V } \\
\text { g } \\
\text { s }\end{array}$ & $\begin{array}{l}17.38 \\
1956 . \\
5.123\end{array}$ & $\begin{array}{l}17.65 \\
1973 . \\
5.153\end{array}$ & $\begin{array}{l}17.92 \\
1989 . \\
5.181\end{array}$ & $\begin{array}{l}18.19 \\
2005 . \\
5.210\end{array}$ & $\begin{array}{l}18.45 \\
2021 . \\
5.238\end{array}$ & $\begin{array}{l}18.71 \\
2037 . \\
5.265\end{array}$ & $\begin{array}{l}18.97 \\
2053 . \\
5.293\end{array}$ & $\begin{array}{l}19.23 \\
2068 . \\
5.319\end{array}$ & $\begin{array}{l}19.48 \\
2084 . \\
5.346\end{array}$ & $\begin{array}{l}19.74 \\
2100 . \\
5.372\end{array}$ & $\begin{array}{l}19.99 \\
2115 . \\
5.398\end{array}$ \\
\hline 13600 & $\begin{array}{l}y \\
\text { s } \\
\text { s }\end{array}$ & $\begin{array}{l}17.08 \\
19 \leqq 4.4 \\
5.112\end{array}$ & $\begin{array}{l}17.35 \\
970 \\
\leq .142\end{array}$ & $\begin{array}{l}17.62 \\
1986 . \\
5.171\end{array}$ & $\begin{array}{l}17.88 \\
2002 . \\
5.199\end{array}$ & $\begin{array}{l}18.14 \\
2019 . \\
5.227\end{array}$ & $\begin{array}{l}18: 40 \\
2034 \\
5.255\end{array}$ & $\begin{array}{l}18.66 \\
2050 . \\
5.282\end{array}$ & $\begin{array}{l}18.91 \\
2066 . \\
5.309\end{array}$ & $\begin{array}{l}19.16 \\
2082 . \\
5.336\end{array}$ & $\begin{array}{l}19.41 \\
2098 . \\
5.362\end{array}$ & $\begin{array}{l}19.66 \\
2113 \\
5.388\end{array}$ \\
\hline 13800 & $\begin{array}{l}\text { v } \\
\mathrm{B} \\
\mathrm{s}\end{array}$ & $\begin{array}{l}36.79 \\
1951 . \\
5.102\end{array}$ & $\begin{array}{l}17.06 \\
1968 \\
\qquad .13 i\end{array}$ & $\begin{array}{l}17.32 \\
1984 . \\
5.160\end{array}$ & $\begin{array}{l}17.58 \\
2000 . \\
5.189\end{array}$ & $\begin{array}{l}17.84 \\
2016 . \\
5.217\end{array}$ & $\begin{array}{l}18.10 \\
2032 . \\
5.245\end{array}$ & $\begin{array}{l}18.35 \\
2008 . \\
5.272\end{array}$ & $\begin{array}{l}18.60 \\
2060 . \\
5.299\end{array}$ & $\begin{array}{l}18.85 \\
2080 . \\
5.326\end{array}$ & $\begin{array}{l}19.10 \\
2095 . \\
5.352\end{array}$ & $\begin{array}{l}19.35 \\
2011 . \\
5.378\end{array}$ \\
\hline 14000 & $\begin{array}{l}\text { V } \\
\text { s } \\
s\end{array}$ & $\begin{array}{l}16.51 \\
1949 . \\
5.091\end{array}$ & $\begin{array}{l}16.77 \\
1965 . \\
5.128\end{array}$ & $\begin{array}{l}17.03 \\
1982 \\
5.150\end{array}$ & $\begin{array}{l}17.29 \\
1998 . \\
5.178\end{array}$ & $\begin{array}{l}17.55 \\
2014 \\
5.207\end{array}$ & $\begin{array}{l}17.80 \\
2030 . \\
5.235\end{array}$ & $\begin{array}{l}18.05 \\
2046 . \\
5.262\end{array}$ & $\begin{array}{l}18.30 \\
2062 . \\
5.289\end{array}$ & $\begin{array}{l}18.55 \\
2078 . \\
5.316\end{array}$ & $\begin{array}{l}18.79 \\
2093 . \\
5.342\end{array}$ & $\begin{array}{l}19.08 \\
2109 . \\
5.369\end{array}$ \\
\hline 19200 & ' & $\begin{array}{l}16.23 \\
1946 . \\
5.080\end{array}$ & $\begin{array}{l}16.50 \\
1963 . \\
5.110\end{array}$ & $\begin{array}{l}16.75 \\
1979 . \\
5.139\end{array}$ & $\begin{array}{l}17.01 \\
1995 . \\
5.168\end{array}$ & $\begin{array}{l}17.26 \\
2002 . \\
5.197\end{array}$ & $\begin{array}{l}87.51 \\
2028 . \\
5.225\end{array}$ & $\begin{array}{l}17.76 \\
2044 . \\
5.252\end{array}$ & $\begin{array}{l}18.01 \\
2060 . \\
5.279\end{array}$ & $\begin{array}{l}18.25 \\
2075 . \\
5.306\end{array}$ & $\begin{array}{l}18.50 \\
2091 . \\
5.333\end{array}$ & $\begin{array}{l}18.74 \\
2107 . \\
5.359\end{array}$ \\
\hline 10400 & $\begin{array}{l}\dot{y} \\
\text { u } \\
5\end{array}$ & $\begin{array}{l}15.97 \\
1904 . \\
5.070\end{array}$ & $\begin{array}{l}16.23 \\
\$ 960 \\
5.100\end{array}$ & $\begin{array}{l}16.48 \\
1977 . \\
5.129\end{array}$ & $\begin{array}{l}16.74 \\
1993 . \\
5.158\end{array}$ & $\begin{array}{l}16.99 \\
2009 . \\
5.187\end{array}$ & $\begin{array}{l}17.23 \\
2025 . \\
5.215\end{array}$ & $\begin{array}{l}17.48 \\
2041 . \\
5.242\end{array}$ & $\begin{array}{l}17.73 \\
2057 \\
5.270\end{array}$ & $\begin{array}{l}17.97 \\
2073 . \\
5.296\end{array}$ & $\begin{array}{l}18.21 \\
2089 . \\
5.323\end{array}$ & $\begin{array}{l}18.45 \\
2105 . \\
5.349\end{array}$ \\
\hline
\end{tabular}


Table 10 (Continued)

Temperature range: 280 tc. $330^{\circ} \mathrm{C}$

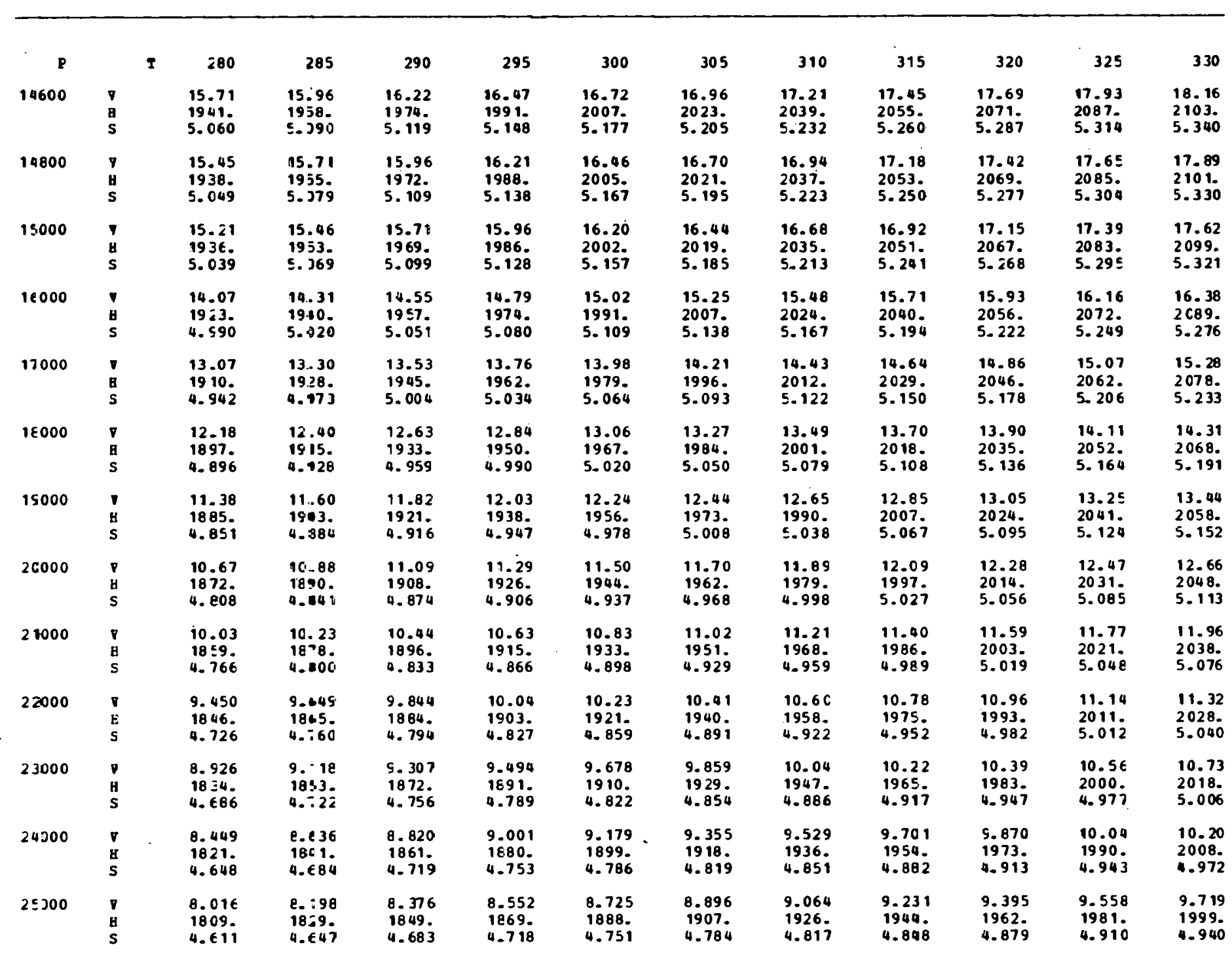


Table 10 (Continued)

Temperature range: 280 to $330^{\circ} \mathrm{C}$

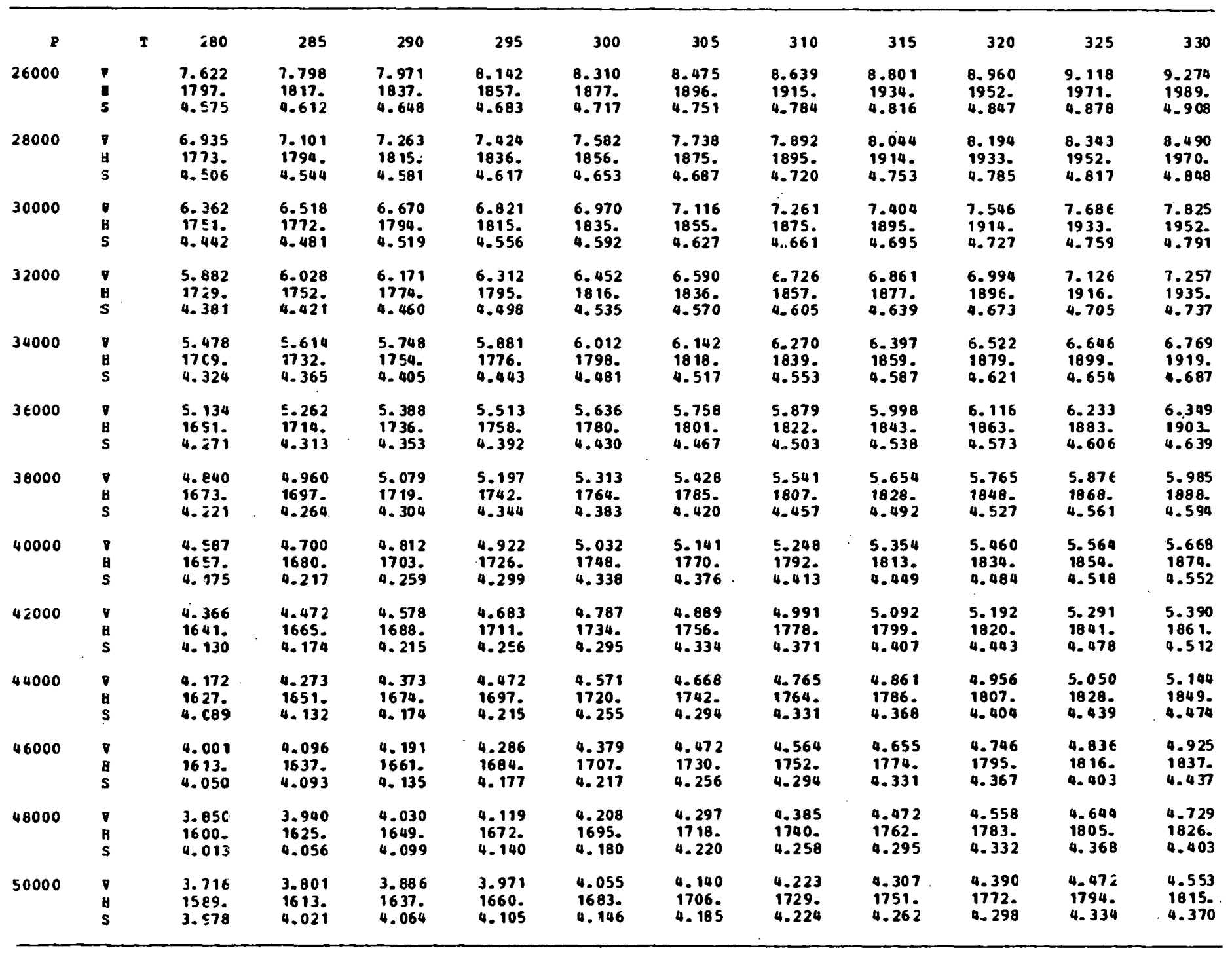


Table 11. The saturation properties of is obutane

\begin{tabular}{|c|c|c|c|c|c|c|c|c|c|c|}
\hline $\mathrm{T}$ & $\mathbf{P}$ & $\mathbf{v}_{\mathbf{f}}$ & $v_{f g}$ & $\mathbf{v}_{\mathrm{g}}$ & $h_{f}$ & ${ }^{h} f_{g}$ & $\mathrm{~h}_{\mathrm{g}}$ & ${ }^{s_{f}}$ & $s_{f g}$ & $\varsigma_{g}$ \\
\hline $\begin{array}{l}-20 \\
-18 \\
-16 \\
-14 \\
-12\end{array}$ & $\begin{array}{c}72.65 \\
78.91 \\
85.59 \\
92.70 \\
100.3\end{array}$ & $\begin{array}{l}1.658 \\
1.664 \\
1.670 \\
1.677 \\
1.683\end{array}$ & $\begin{array}{l}479.7 \\
444.0 \\
411.6 \\
382.0 \\
355.0\end{array}$ & $\begin{array}{l}481.3 \\
445.7 \\
413.2 \\
383.6 \\
356.6\end{array}$ & $\begin{array}{l}40.17 \\
44.34 \\
48.51 \\
52.67 \\
56.83\end{array}$ & $\begin{array}{l}367.7 \\
366.1 \\
364.5 \\
363.0 \\
361.4\end{array}$ & $\begin{array}{l}407.8 \\
410.4 \\
413.0 \\
415.7 \\
418.3\end{array}$ & $\begin{array}{l}0.1650 \\
0.1813 \\
0.1975 \\
0.2136 \\
0.2296\end{array}$ & $\begin{array}{l}1.452 \\
1.435 \\
1.418 \\
1.401 \\
1.384\end{array}$ & $\begin{array}{l}1.617 \\
1.616 \\
1.615 \\
1.614 \\
1.614\end{array}$ \\
\hline-11.73 & 101.3 & 1.684 & 351.3 & 353.0 & 57.55 & 361.1 & 418.6 & 0.2323 & 1.381 & 1.614 \\
\hline $\begin{array}{l}-10 \\
-8 \\
-6 \\
-4 \\
-\quad 2\end{array}$ & $\begin{array}{l}108.3 \\
116.8 \\
125.9 \\
135.5 \\
145.6\end{array}$ & $\begin{array}{l}1.690 \\
1.696 \\
1.703 \\
1.710 \\
1.716\end{array}$ & $\begin{array}{r}330.0 \\
307.5 \\
286.5 \\
267.3 \\
249.8\end{array}$ & $\begin{array}{l}331.7 \\
309.1 \\
288.2 \\
269.1 \\
251.5\end{array}$ & $\begin{array}{l}61.25 \\
65.40 \\
69.81 \\
74.21 \\
78.48\end{array}$ & $\begin{array}{l}359.6 \\
358.1 \\
356.3 \\
354.6 \\
353.0\end{array}$ & $\begin{array}{l}420.9 \\
423.5 \\
426.2 \\
428.8 \\
431.4\end{array}$ & $\begin{array}{l}0.2464 \\
0.2621 \\
0.2785 \\
0.2949 \\
0.3106\end{array}$ & $\begin{array}{l}1.367 \\
1.351 \\
1.334 \\
1.317 \\
1.302\end{array}$ & $\begin{array}{l}1.613 \\
1.613 \\
1.612 \\
1.512 \\
1.512\end{array}$ \\
\hline 0 & 156.3 & 1.723 & 233.6 & 235.3 & 82.87 & 351.2 & 434.1 & 0.3267 & 1.286 & 1.513 \\
\hline $\begin{array}{r}2 \\
4 \\
6 \\
8 \\
10\end{array}$ & $\begin{array}{l}167.7 \\
179.6 \\
192.2 \\
205.4 \\
219.3\end{array}$ & $\begin{array}{l}1.730 \\
1.737 \\
1.745 \\
1.752 \\
1.759\end{array}$ & $\begin{array}{l}218.7 \\
204.8 \\
192.1 \\
180.2 \\
169.3\end{array}$ & $\begin{array}{l}220.4 \\
206.5 \\
193.3 \\
131.9 \\
171.1\end{array}$ & $\begin{array}{c}87.26 \\
91.77 \\
96.15 \\
100.8 \\
105.1\end{array}$ & $\begin{array}{l}349.5 \\
347.6 \\
345.9 \\
343.9 \\
342.2\end{array}$ & $\begin{array}{l}436.7 \\
439.4 \\
442.1 \\
444.7 \\
447.4\end{array}$ & $\begin{array}{l}0.3427 \\
0.3589 \\
0.3746 \\
0.3910 \\
0.4064\end{array}$ & $\begin{array}{l}1.270 \\
1.254 \\
1.239 \\
1.223 \\
1.209\end{array}$ & $\begin{array}{l}1.513 \\
1.013 \\
1.614 \\
1.614 \\
1.615\end{array}$ \\
\hline $\begin{array}{l}12 \\
14 \\
16 \\
18 \\
20\end{array}$ & $\begin{array}{l}234.0 \\
249.4 \\
265.5 \\
282.4 \\
300.1\end{array}$ & $\begin{array}{l}1.767 \\
1.774 \\
1.782 \\
1.789 \\
1.797\end{array}$ & $\begin{array}{l}159.1 \\
149.7 \\
140.9 \\
132.8 \\
125.2\end{array}$ & $\begin{array}{l}160.7 \\
151.5 \\
142.7 \\
134.6 \\
127.0\end{array}$ & $\begin{array}{l}109.7 \\
114.4 \\
118.9 \\
123.5 \\
128.1\end{array}$ & $\begin{array}{l}340.3 \\
338.4 \\
336.4 \\
334.5 \\
332.6\end{array}$ & $\begin{array}{l}450.0 \\
45.2 .7 \\
45.5 .4 \\
458.0 \\
460.7\end{array}$ & $\begin{array}{l}0.4225 \\
0.4385 \\
0.4543 \\
0.4701 \\
0.4857\end{array}$ & $\begin{array}{l}1.193 \\
1.178 \\
1.163 \\
1.149 \\
1.135\end{array}$ & $\begin{array}{l}1.616 \\
1.617 \\
1.618 \\
1.619 \\
1.620\end{array}$ \\
\hline $\begin{array}{l}22 \\
24 \\
26 \\
28 \\
30\end{array}$ & $\begin{array}{l}318.7 \\
338.1 \\
358.3 \\
379.5 \\
401.6\end{array}$ & $\begin{array}{l}1.805 \\
1.813 \\
1.822 \\
1.830 \\
1.838\end{array}$ & $\begin{array}{l}118.1 \\
-11.5 \\
-05.3 \\
99.51 \\
94.11\end{array}$ & $\begin{array}{c}119.9 \\
113.3 \\
107.1 \\
101.3 \\
95.94\end{array}$ & $\begin{array}{l}133.0 \\
137.5 \\
142.3 \\
147.1 \\
151.9\end{array}$ & $\begin{array}{l}330.4 \\
328.15 \\
326.4 \\
324.2 \\
322.1\end{array}$ & $\begin{array}{l}463.4 \\
466.0 \\
468.7 \\
471.4 \\
474.0\end{array}$ & $\begin{array}{l}0.5019 \\
0.5173 \\
0.5333 \\
0.5491 \\
0.5649\end{array}$ & $\begin{array}{l}1.119 \\
1.106 \\
1.091 \\
1.077 \\
1.062\end{array}$ & $\begin{array}{l}1 . \in 21 \\
1 . \in 23 \\
1 . \in 24 \\
1 . \in 26 \\
1 . \in 27\end{array}$ \\
\hline
\end{tabular}


Table 11 (Continued)

\begin{tabular}{|c|c|c|c|c|c|c|c|c|c|c|}
\hline $\mathrm{T}$ & $\mathbf{P}$ & $v_{f}$ & $v_{f g}$ & $\mathbf{v}_{\mathbf{g}}$ & $h_{f}$ & $h_{f g}$ & $h_{g}$ & $s_{f}$ & $s_{f g}$ & $\mathbf{s}_{\mathbf{g}}$ \\
\hline $\begin{array}{l}32 \\
34 \\
36 \\
38 \\
40\end{array}$ & $\begin{array}{l}424.7 \\
448.8 \\
473.8 \\
499.9 \\
527.1\end{array}$ & $\begin{array}{l}1.847 \\
1.856 \\
1.865 \\
1.874 \\
1.883\end{array}$ & $\begin{array}{l}89.05 \\
84.32 \\
79.90 \\
75.69 \\
71.77\end{array}$ & $\begin{array}{l}90.90 \\
86.18 \\
81.76 \\
77.56 \\
73.65\end{array}$ & $\begin{array}{l}156.7 \\
161.5 \\
166.3 \\
171.3 \\
176.2\end{array}$ & $\begin{array}{l}319.9 \\
317.8 \\
315.7 \\
313.3 \\
311.1\end{array}$ & $\begin{array}{l}476.7 \\
479.3 \\
482.0 \\
484.6 \\
487.3\end{array}$ & $\begin{array}{l}0.5805 \\
0.5960 \\
0.6114 \\
0.6273 \\
0.6428\end{array}$ & $\begin{array}{l}1.048 \\
1.035 \\
1.021 \\
1.007 \\
0.9934\end{array}$ & $\begin{array}{l}1.629 \\
1.631 \\
1.633 \\
1.634 \\
1.636\end{array}$ \\
\hline $\begin{array}{l}42 \\
44 \\
46 \\
48 \\
50\end{array}$ & $\begin{array}{l}555.3 \\
584.7 \\
615.2 \\
646.8 \\
679.7\end{array}$ & $\begin{array}{l}1.893 \\
1.902 \\
1.912 \\
1.922 \\
1.932\end{array}$ & $\begin{array}{l}58.09 \\
54.61 \\
61.33 \\
58.20 \\
55.31\end{array}$ & $\begin{array}{l}69.98 \\
66.51 \\
63.24 \\
60.13 \\
57.24\end{array}$ & $\begin{array}{l}181.1 \\
186.1 \\
191.0 \\
196.3 \\
201.2\end{array}$ & $\begin{array}{l}308.8 \\
306.5 \\
304.1 \\
301.5 \\
299.1\end{array}$ & $\begin{array}{l}489.9 \\
492.5 \\
495.1 \\
497.7 \\
500.3\end{array}$ & $\begin{array}{l}0.6582 \\
0.6738 \\
0.6893 \\
0.7054 \\
0.7207\end{array}$ & $\begin{array}{l}0.9799 \\
0.9663 \\
0.9528 \\
0.9387 \\
0.9256\end{array}$ & $\begin{array}{l}1.638 \\
1.640 \\
1.642 \\
1.644 \\
1.646\end{array}$ \\
\hline $\begin{array}{l}52 \\
54 \\
56 \\
58 \\
60\end{array}$ & $\begin{array}{l}713.8 \\
749.2 \\
785.8 \\
823.8 \\
863.1\end{array}$ & $\begin{array}{l}1.943 \\
1.954 \\
1.964 \\
1.976 \\
1.987\end{array}$ & $\begin{array}{l}52.54 \\
49.95 \\
47.48 \\
45.14 \\
42.94\end{array}$ & $\begin{array}{l}54.48 \\
51.90 \\
49.45 \\
47.11 \\
44.93\end{array}$ & $\begin{array}{l}206.4 \\
211.5 \\
216.7 \\
222.0 \\
227.2\end{array}$ & $\begin{array}{l}296.5 \\
294.0 \\
291.4 \\
288.6 \\
286.0\end{array}$ & $\begin{array}{l}502.9 \\
505.5 \\
508.1 \\
510.6 \\
513.2\end{array}$ & $\begin{array}{l}0.7365 \\
0.7519 \\
0.7675 \\
0.7833 \\
0.7987\end{array}$ & $\begin{array}{l}0.9118 \\
0.8986 \\
0.8852 \\
0.8715 \\
0.8584\end{array}$ & $\begin{array}{l}1.648 \\
1.650 \\
1.653 \\
1.655 \\
1.657\end{array}$ \\
\hline $\begin{array}{l}62 \\
64 \\
66 \\
68 \\
70\end{array}$ & $\begin{array}{c}903.8 \\
945.8 \\
989.4 \\
1034 \\
1081\end{array}$ & $\begin{array}{l}1.999 \\
2.011 \\
2.024 \\
2.036 \\
2.050\end{array}$ & $\begin{array}{l}40.87 \\
38.87 \\
36.98 \\
35.19 \\
33.50\end{array}$ & $\begin{array}{l}42.87 \\
40.88 \\
39.00 \\
37.23 \\
35.55\end{array}$ & $\begin{array}{l}232.4 \\
237.8 \\
243.2 \\
248.6 \\
254.0\end{array}$ & $\begin{array}{l}283.3 \\
280.4 \\
277.5 \\
274.6 \\
271.6\end{array}$ & $\begin{array}{l}515.7 \\
518.2 \\
520.7 \\
523.1 \\
525.6\end{array}$ & $\begin{array}{l}0.8139 \\
0.8297 \\
0.8454 \\
0.8610 \\
0.8765\end{array}$ & $\begin{array}{l}0.8454 \\
0.8317 \\
0.8182 \\
0.8049 \\
0.7916\end{array}$ & $\begin{array}{l}1.659 \\
1.661 \\
1.664 \\
1.666 \\
1.668\end{array}$ \\
\hline $\begin{array}{l}72 \\
74 \\
76 \\
78 \\
80\end{array}$ & $\begin{array}{l}1129 \\
1179 \\
1230 \\
1283 \\
1337\end{array}$ & $\begin{array}{l}2.063 \\
2.077 \\
2.092 \\
2.107 \\
2.122\end{array}$ & $\begin{array}{l}31.87 \\
30.32 \\
28.85 \\
27.45 \\
26.13\end{array}$ & $\begin{array}{l}33.93 \\
32.39 \\
30.94 \\
29.56 \\
28.25\end{array}$ & $\begin{array}{l}259.6 \\
265.2 \\
270.8 \\
276.4 \\
282.0\end{array}$ & $\begin{array}{l}268.4 \\
265.2 \\
262.0 \\
258.7 \\
255.5\end{array}$ & $\begin{array}{l}528.0 \\
530.4 \\
532.8 \\
535.1 \\
537.4\end{array}$ & $\begin{array}{l}0.8925 \\
0.9084 \\
0.9242 \\
0.9399 \\
0.9556\end{array}$ & $\begin{array}{l}0.7777 \\
0.7640 \\
0.7503 \\
0.7368 \\
0.7233\end{array}$ & $\begin{array}{l}1.670 \\
1.672 \\
1.675 \\
1.677 \\
1.679\end{array}$ \\
\hline $\begin{array}{l}82 \\
84 \\
86 \\
88 \\
90\end{array}$ & $\begin{array}{l}1393 \\
1451 \\
1511 \\
1573 \\
1636\end{array}$ & $\begin{array}{l}2.138 \\
2.155 \\
2.172 \\
2.191 \\
2.209\end{array}$ & $\begin{array}{l}24.84 \\
23.62 \\
22.45 \\
21.34 \\
20.26\end{array}$ & $\begin{array}{l}26.98 \\
25.78 \\
24.62 \\
23.53 \\
22.47\end{array}$ & $\begin{array}{l}287.8 \\
293.6 \\
299.6 \\
305.5 \\
311.6\end{array}$ & $\begin{array}{l}251.9 \\
248.3 \\
244.6 \\
240.8 \\
236.9\end{array}$ & $\begin{array}{l}539.7 \\
542.0 \\
544.1 \\
546.3 \\
548.4\end{array}$ & $\begin{array}{l}0.9717 \\
0.9877 \\
1.004 \\
1.020 \\
1.036\end{array}$ & $\begin{array}{l}0.7093 \\
0.6953 \\
0.6810 \\
0.6667 \\
0.6522\end{array}$ & $\begin{array}{l}1.681 \\
1.683 \\
1.685 \\
1.688 \\
1.688\end{array}$ \\
\hline
\end{tabular}


Table 11 (Continued)

\begin{tabular}{|c|c|c|c|c|c|c|c|c|c|c|}
\hline $\mathrm{T}$ & $\mathbf{P}$ & $v_{f}$ & $v_{f g}$ & $\mathbf{v}_{\mathbf{g}}$ & $\mathrm{h}_{\mathbf{f}}$ & $h_{E g}$ & $\mathrm{~h}_{\mathrm{g}}$ & $s_{f}$ & $\mathbf{s}_{\mathrm{fg}}$ & $\mathbf{s}_{\mathrm{g}}$ \\
\hline $\begin{array}{r}92 \\
94 \\
96 \\
98 \\
100\end{array}$ & $\begin{array}{l}1702 \\
1769 \\
1838 \\
1909 \\
1983\end{array}$ & $\begin{array}{l}2.229 \\
2.250 \\
2.272 \\
2.295 \\
2.319\end{array}$ & $\begin{array}{l}19.24 \\
18.25 \\
17.30 \\
16.37 \\
15.49\end{array}$ & $\begin{array}{l}21.47 \\
20.50 \\
19.57 \\
18.67 \\
17.81\end{array}$ & $\begin{array}{l}317.6 \\
323.9 \\
330.1 \\
336.6 \\
343.1\end{array}$ & $\begin{array}{l}232.9 \\
228.6 \\
224.3 \\
219.6 \\
214.9\end{array}$ & $\begin{array}{l}550.5 \\
552.5 \\
554.4 \\
556.2 \\
558.0\end{array}$ & $\begin{array}{l}1.052 \\
1.069 \\
1.086 \\
1.103 \\
1.120\end{array}$ & $\begin{array}{l}0.6378 \\
0.6226 \\
0.6076 \\
0.5918 \\
0.5760\end{array}$ & $\begin{array}{l}1.690 \\
1.692 \\
1.693 \\
1.695 \\
1.696\end{array}$ \\
\hline $\begin{array}{l}102 \\
104 \\
106 \\
108 \\
110\end{array}$ & $\begin{array}{l}2058 \\
2136 \\
2216 \\
2298 \\
2382\end{array}$ & $\begin{array}{l}2.345 \\
2.372 \\
2.401 \\
2.432 \\
2.466\end{array}$ & $\begin{array}{l}14.65 \\
13.83 \\
13.04 \\
12.25 \\
11.52\end{array}$ & $\begin{array}{l}16.99 \\
16.20 \\
15.44 \\
14.69 \\
13.98\end{array}$ & $\begin{array}{l}349.6 \\
356.3 \\
363.0 \\
370.1 \\
377.1\end{array}$ & $\begin{array}{l}210.2 \\
205.1 \\
199.9 \\
194.1 \\
188.5\end{array}$ & $\begin{array}{l}559.8 \\
561.4 \\
562.9 \\
564.2 \\
565.6\end{array}$ & $\begin{array}{l}1.137 \\
1.154 \\
1.171 \\
1.189 \\
1.207\end{array}$ & $\begin{array}{l}0.5603 \\
0.5438 \\
0.5273 \\
0.5093 \\
0.4919\end{array}$ & $\begin{array}{l}1.697 \\
1.698 \\
1.699 \\
1.699 \\
1.699\end{array}$ \\
\hline $\begin{array}{l}112 \\
114 \\
116 \\
118 \\
120\end{array}$ & $\begin{array}{l}2469 \\
2558 \\
2649 \\
2743 \\
2839\end{array}$ & $\begin{array}{l}2.503 \\
2.542 \\
2.586 \\
2.635 \\
2.689\end{array}$ & $\begin{array}{c}10.78 \\
10.05 \\
9.359 \\
8.660 \\
7.972\end{array}$ & $\begin{array}{l}13.28 \\
12.60 \\
11.94 \\
11.29 \\
10.66\end{array}$ & $\begin{array}{l}384.4 \\
392.0 \\
399.6 \\
407.6 \\
415.8\end{array}$ & $\begin{array}{l}182.2 \\
175.5 \\
168.7 \\
161.2 \\
153.1\end{array}$ & $\begin{array}{l}566.6 \\
567.5 \\
568.3 \\
568.7 \\
568.9\end{array}$ & $\begin{array}{l}1.226 \\
1.245 \\
1.264 \\
1.284 \\
1.304\end{array}$ & $\begin{array}{l}0.4731 \\
0.4534 \\
0.4335 \\
0.4120 \\
0.3895\end{array}$ & $\begin{array}{l}1.699 \\
1.698 \\
1.697 \\
1.696 \\
1.693\end{array}$ \\
\hline $\begin{array}{l}122 \\
124 \\
126 \\
128 \\
130\end{array}$ & $\begin{array}{l}2938 \\
3040 \\
3144 \\
3252 \\
3362\end{array}$ & $\begin{array}{l}2.751 \\
2.823 \\
2.909 \\
3.013 \\
3.149\end{array}$ & $\begin{array}{l}7.293 \\
6.594 \\
5.861 \\
5.114 \\
4.295\end{array}$ & $\begin{array}{c}10.04 \\
9.418 \\
8.769 \\
8.127 \\
7.444\end{array}$ & $\begin{array}{l}424.3 \\
433.3 \\
443.1 \\
453.4 \\
464.8\end{array}$ & $\begin{array}{c}144.6 \\
134.8 \\
123.6 \\
111.3 \\
96.37\end{array}$ & $\begin{array}{l}568.8 \\
568.2 \\
566.7 \\
564.6 \\
561.2\end{array}$ & $\begin{array}{l}1.325 \\
1.347 \\
1.371 \\
1.395 \\
1.423\end{array}$ & $\begin{array}{l}0.3658 \\
0.3395 \\
0.3097 \\
0.2774 \\
0.2390\end{array}$ & $\begin{array}{l}1.690 \\
1.686 \\
1.680 \\
1.673 \\
1.662\end{array}$ \\
\hline $\begin{array}{l}132 \\
134 \\
135\end{array}$ & $\begin{array}{l}3474 \\
3590 \\
3648\end{array}$ & $\begin{array}{l}3.343 \\
3.698 \\
4.522\end{array}$ & $\begin{array}{l}3.334 \\
1.977 \\
0.0\end{array}$ & $\begin{array}{l}6.677 \\
5.675 \\
4.522\end{array}$ & $\begin{array}{l}478.1 \\
496.0 \\
520.0\end{array}$ & $\begin{array}{c}77.14 \\
47.17 \\
0.0\end{array}$ & $\begin{array}{l}555.3 \\
543.1 \\
520.0\end{array}$ & $\begin{array}{l}1.455 \\
1.498 \\
1.556\end{array}$ & $\begin{array}{l}0.1904 \\
0.1159 \\
0.0\end{array}$ & $\begin{array}{l}1.646 \\
1.614 \\
1.556\end{array}$ \\
\hline
\end{tabular}


Table 12. Thermodynamic properties of isobutane in the superheated vapor state Temperature range: -40 to $60^{\circ} \mathrm{C}$

\begin{tabular}{|c|c|c|c|c|c|c|c|c|c|c|c|c|}
\hline & & & & & & & & & - & & & \\
\hline p & $T$ & -40 & -30 & -20 & -10 & 0 & 10 & 20 & 30 & 40 & 50 & 60 \\
\hline 5 & $\begin{array}{l}\mathrm{V} \\
\mathrm{H} \\
\mathrm{S}\end{array}$ & $\begin{array}{l}6650 . \\
384.0 \\
1.897\end{array}$ & $\begin{array}{l}6940 . \\
397.6 \\
1.954\end{array}$ & $\begin{array}{l}7230 \\
411.7 \\
2.011\end{array}$ & $\begin{array}{l}7510 . \\
426.3 \\
2.067\end{array}$ & $\begin{array}{l}7800 . \\
44.5 \\
2.124\end{array}$ & $\begin{array}{l}8090 . \\
457.2 \\
2.180\end{array}$ & $\begin{array}{l}8380 . \\
473.3 \\
2.237\end{array}$ & $\begin{array}{l}8660 . \\
490.0 \\
2.293\end{array}$ & $\begin{array}{l}8950 . \\
507.2 \\
2.348\end{array}$ & $\begin{array}{l}9240 . \\
524.9 \\
2.404\end{array}$ & $\begin{array}{l}9520 . \\
543.1 \\
2.459\end{array}$ \\
\hline 50 & $\begin{array}{l}\mathrm{H} \\
\mathrm{H} \\
\mathrm{S}\end{array}$ & & & $\begin{array}{l}707.4 \\
409.2 \\
1.674\end{array}$ & $\begin{array}{l}737.5 \\
424.0 \\
1.732\end{array}$ & $\begin{array}{l}767.4 \\
439.4 \\
1.789\end{array}$ & $\begin{array}{l}797.2 \\
455.2 \\
1.846\end{array}$ & $\begin{array}{l}826.8 \\
471.6 \\
1.903\end{array}$ & $\begin{array}{l}856.3 \\
488.4 \\
1.959\end{array}$ & $\begin{array}{l}885.8 \\
505.7 \\
2.016\end{array}$ & $\begin{array}{l}915.1 \\
523.5 \\
2.071\end{array}$ & $\begin{array}{l}944.4 \\
541.7 \\
2.127\end{array}$ \\
\hline 100 & $\begin{array}{l}\mathrm{V} \\
\mathrm{H} \\
\mathrm{S}\end{array}$ & & & & $\begin{array}{l}360.7 \\
421.4 . \\
1.6 .26\end{array}$ & $\begin{array}{l}376.4 \\
436.9 \\
1.684\end{array}$ & $\begin{array}{l}391.9 \\
453.0 \\
1.742\end{array}$ & $\begin{array}{l}407.3 \\
469.5 \\
1.799\end{array}$ & $\begin{array}{l}422.5 \\
486.5 \\
1.856\end{array}$ & $\begin{array}{l}437.7 \\
504.0 \\
1.913\end{array}$ & $\begin{array}{l}452.7 \\
521.9 \\
1.969\end{array}$ & $\begin{array}{l}467.7 \\
540.2 \\
2.025\end{array}$ \\
\hline 101.33 & $\begin{array}{l}1 \\
: \\
s\end{array}$ & & & . & $\begin{array}{l}355.8 \\
421.3 \\
1.624\end{array}$ & $\begin{array}{l}371.3 \\
436.9 \\
1.682\end{array}$ & $\begin{array}{l}386.6 \\
45.9 \\
1.740\end{array}$ & $\begin{array}{l}401.8 \\
469.5 \\
1.797\end{array}$ & $\begin{array}{l}416.9 \\
48.4 \\
1.854\end{array}$ & $\begin{array}{l}431.8 \\
503.9 \\
1.911\end{array}$ & $\begin{array}{l}446.7 \\
521.8 \\
1.967\end{array}$ & $\begin{array}{l}461.5 \\
540.2 \\
2.023\end{array}$ \\
\hline 200 & $\begin{array}{l}\mathrm{s} \\
\mathrm{z} \\
\mathrm{y}\end{array}$ & & & . & & & $\begin{array}{l}189.0 \\
448.3 \\
1.631\end{array}$ & $\begin{array}{l}197.3 \\
465.3 \\
1.689\end{array}$ & $\begin{array}{l}205.5 \\
482.6 \\
1.748\end{array}$ & $\begin{array}{l}213.5 \\
500.4 \\
1.805\end{array}$ & $\begin{array}{l}221.4 \\
518.6 \\
1.862\end{array}$ & $\begin{array}{l}229.3 \\
537.2 \\
1.919\end{array}$ \\
\hline 300 & $\begin{array}{l}\text { B } \\
\text { s }\end{array}$ & & & & & & & $\begin{array}{l}127.0 \\
460.7 \\
1.620\end{array}$ & $\begin{array}{l}132.9 \\
478.5 \\
1.680\end{array}$ & $\begin{array}{l}138.6 \\
496.6 \\
1.739\end{array}$ & $\begin{array}{l}144.2 \\
515.1 \\
1.797\end{array}$ & $\begin{array}{l}149.7 \\
534.0 \\
1.854\end{array}$ \\
\hline 400 & i & & & & & & & & $\begin{array}{l}96.42 \\
474.1 \\
1.628\end{array}$ & $\begin{array}{l}101.0 \\
492.6 \\
1.688\end{array}$ & $\begin{array}{l}105.5 \\
511.5 \\
1.748\end{array}$ & $\begin{array}{l}109.8 \\
530.7 \\
1.806\end{array}$ \\
\hline 500 & $\begin{array}{l}7 \\
4 \\
5\end{array}$ & & & & & & & & & $\begin{array}{l}78.33 \\
488.4 \\
1.647\end{array}$ & $\begin{array}{l}82.15 \\
507.7 \\
1.707\end{array}$ & $\begin{array}{l}85.83 \\
527.3 \\
1.767\end{array}$ \\
\hline 600 & $\begin{array}{l}\mathrm{Y} \\
\mathrm{H} \\
\mathrm{S}\end{array}$ & & & & & & & & & & $\begin{array}{l}66.49 \\
503.7 \\
1.672\end{array}$ & $\begin{array}{l}69.76 \\
523.6 \\
1.733\end{array}$ \\
\hline 700 & $\begin{array}{l}\nabla \\
H \\
S\end{array}$ & & & & & & . & & & & $\cdots$ & $\begin{array}{l}58.20 \\
519.9 \\
1.702\end{array}$ \\
\hline 800 & $\begin{array}{l}\mathbf{v} \\
\mathbf{H} \\
\mathbf{S}\end{array}$ & & & & & & & & & & . & $\begin{array}{l}49.45 \\
515.8 \\
1.674\end{array}$ \\
\hline 900 & $\begin{array}{l}\mathbf{I} \\
\mathrm{H} \\
\mathrm{S}\end{array}$ & & & & & & & & & & & \\
\hline 1000 & $\begin{array}{l}\mathrm{V} \\
\mathrm{H} \\
\mathrm{S}\end{array}$ & & & & & & & & & & & \\
\hline
\end{tabular}


Table 12 (Continued)

Temperature range: 70 to $170^{\circ} \mathrm{C}$

\begin{tabular}{|c|c|c|c|c|c|c|c|c|c|c|c|c|}
\hline $\mathbf{P}$ & $\mathbf{T}$ & 70 & 80 & 90 & 100 & 110 & 120 & 130 & 140 & 150 & 160 & 170 \\
\hline 5 & $\begin{array}{l}V \\
H \\
S\end{array}$ & $\begin{array}{l}9810 \\
561.7 \\
2.514\end{array}$ & $\begin{array}{r}101 C 0 . \\
58 c .8 \\
2.569\end{array}$ & $\begin{array}{c}10380 . \\
600.4 \\
2.624\end{array}$ & $\begin{array}{r}10 E 70 . \\
620.4 \\
2.678\end{array}$ & $\begin{array}{r}10960 . \\
640.9 \\
2.733\end{array}$ & $\begin{array}{r}11240 . \\
661.9 \\
2.787\end{array}$ & $\begin{array}{r}11530 . \\
683.3 \\
2.840\end{array}$ & $\begin{array}{r}11820 \\
705.1 \\
2.894\end{array}$ & $\begin{array}{r}12100 \\
727.4 \\
2.947\end{array}$ & $\begin{array}{c}12390 \\
750.1 \\
3.000\end{array}$ & $\begin{array}{r}12670 . \\
773.2 \\
3.053\end{array}$ \\
\hline 50 & $\begin{array}{l}7 \\
H \\
5\end{array}$ & $\begin{array}{l}973.6 \\
562.5 \\
2.182\end{array}$ & $\begin{array}{l}1000 . \\
579.7 \\
2.238\end{array}$ & $\begin{array}{l}1030 . \\
599.3 \\
2.293\end{array}$ & $\begin{array}{l}1060 . \\
6 \times 9.4 \\
2.347\end{array}$ & $\begin{array}{l}1090 . \\
640.0 \\
2.402\end{array}$ & $\begin{array}{l}1120 . \\
661.0 \\
2.456\end{array}$ & $\begin{array}{l}1150 . \\
682.4 \\
2.509\end{array}$ & $\begin{array}{l}1180 . \\
704.3 \\
2.563\end{array}$ & $\begin{array}{l}1210 . \\
726.6 \\
2.616\end{array}$ & $\begin{array}{l}1230 . \\
749.3 \\
2.669\end{array}$ & $\begin{array}{l}1260 . \\
772.5 \\
2.722\end{array}$ \\
\hline 100 & $\begin{array}{l}y \\
H \\
S\end{array}$ & $\begin{array}{l}482.6 \\
553.1 \\
2.381\end{array}$ & $\begin{array}{l}497.5 \\
578.4 \\
2.106\end{array}$ & $\begin{array}{l}512.3 \\
598.1 \\
2.191\end{array}$ & $\begin{array}{l}527.1 \\
618.3 \\
2.246\end{array}$ & $\begin{array}{l}541.8 \\
638.9 \\
2.300\end{array}$ & $\begin{array}{l}556.5 \\
660.0 \\
2.355\end{array}$ & $\begin{array}{l}571.1 \\
681.5 \\
2.409\end{array}$ & $\begin{array}{l}585.7 \\
703.4 \\
2.462\end{array}$ & $\begin{array}{l}600.3 \\
725.8 \\
2.516\end{array}$ & $\begin{array}{l}614.9 \\
748.5 \\
2.569\end{array}$ & $\begin{array}{l}629.5 \\
771.7 \\
2.622\end{array}$ \\
\hline 101.33 & $\begin{array}{l}y \\
4 \\
5\end{array}$ & $\begin{array}{l}475.2 \\
55.3 .0 \\
2.579\end{array}$ & $\begin{array}{l}490.9 \\
578.3 \\
2.134\end{array}$ & $\begin{array}{l}505.5 \\
598.1 \\
2.189\end{array}$ & $\begin{array}{l}520.0 \\
618.3 \\
2.244\end{array}$ & $\begin{array}{l}534.6 \\
638.9 \\
2.298\end{array}$ & $\begin{array}{l}549.1 \\
660.0 \\
2.353\end{array}$ & $\begin{array}{l}563.5 \\
681.5 \\
2.407\end{array}$ & $\begin{array}{l}578.0 \\
703.4 \\
2.460\end{array}$ & $\begin{array}{l}592.4 \\
725.7 \\
2.514\end{array}$ & $\begin{array}{l}606.8 \\
748.5 \\
2.567\end{array}$ & $\begin{array}{l}621.1 \\
771.7 \\
2.620\end{array}$ \\
\hline 200 & $\begin{array}{l}V \\
H \\
S\end{array}$ & $\begin{array}{l}237.1 \\
555.2 \\
1.076\end{array}$ & $\begin{array}{l}244.8 \\
575.7 \\
2.031\end{array}$ & $\begin{array}{l}252.5 \\
595.6 \\
2.087\end{array}$ & $\begin{array}{l}263.1 \\
615.0 \\
2.142\end{array}$ & $\begin{array}{l}267.7 \\
636.8 \\
2.197\end{array}$ & $\begin{array}{l}275.2 \\
658.0 \\
2.252\end{array}$ & $\begin{array}{l}282.7 \\
679.6 \\
2.306\end{array}$ & $\begin{array}{l}290.2 \\
701.6 \\
2.360\end{array}$ & $\begin{array}{l}297.6 \\
724.1 \\
2.414\end{array}$ & $\begin{array}{l}305.1 \\
746.9 \\
2.467\end{array}$ & $\begin{array}{l}312.5 \\
770.2 \\
2.520\end{array}$ \\
\hline 300 & $\begin{array}{l}7 \\
H \\
S\end{array}$ & $\begin{array}{l}155.1 \\
55] .3 \\
1.911\end{array}$ & $\begin{array}{l}160.5 \\
573.0 \\
1.958\end{array}$ & $\begin{array}{l}165.8 \\
593.1 \\
2.024\end{array}$ & $\begin{array}{l}171.1 \\
613.6 \\
2.080\end{array}$ & $\begin{array}{l}176.3 \\
634.6 \\
2.135\end{array}$ & $\begin{array}{l}181.4 \\
655.9 \\
2.190\end{array}$ & $\begin{array}{l}186.6 \\
677.6 \\
2.245\end{array}$ & $\begin{array}{l}191.6 \\
699.8 \\
2.299\end{array}$ & $\begin{array}{l}196.7 \\
722.3 \\
2.353\end{array}$ & $\begin{array}{l}201.8 \\
745.3 \\
2.407\end{array}$ & $\begin{array}{l}206.8 \\
768.6 \\
2.460\end{array}$ \\
\hline 5.00 & $\begin{array}{l}y \\
8 \\
5\end{array}$ & $\begin{array}{l}114 .-1 \\
550.3 \\
1.064\end{array}$ & $\begin{array}{l}118.3 \\
570.2 \\
1.9 ? 1\end{array}$ & $\begin{array}{l}122.4 \\
590.5 \\
1.978\end{array}$ & $\begin{array}{l}125.5 \\
611.2 \\
2.034\end{array}$ & $\begin{array}{l}130.5 \\
632.3 \\
2.090\end{array}$ & $\begin{array}{l}134.5 \\
653.8 \\
2.145\end{array}$ & $\begin{array}{l}138.4 \\
675.6 \\
2.200\end{array}$ & $\begin{array}{l}142.4 \\
697.9 \\
2.255\end{array}$ & $\begin{array}{l}146.2 \\
720.6 \\
2.309\end{array}$ & $\begin{array}{l}150.1 \\
743.6 \\
2.363\end{array}$ & $\begin{array}{l}154.0 \\
767.1 \\
2.416\end{array}$ \\
\hline 500 & $\begin{array}{l}1 \\
8 \\
5\end{array}$ & $\begin{array}{l}89.42 \\
54 .-1 \\
1.825\end{array}$ & $\begin{array}{l}92.72 \\
567.3 \\
1.833\end{array}$ & $\begin{array}{l}96.36 \\
587.8 \\
1.941\end{array}$ & $\begin{array}{l}99.73 \\
608.7 \\
1.997\end{array}$ & $\begin{array}{l}103.1 \\
630.0 \\
2.054\end{array}$ & $\begin{array}{l}106.3 \\
651.6 \\
2.109\end{array}$ & $\begin{array}{l}109.6 \\
673.6 \\
2.165\end{array}$ & $\begin{array}{l}112.8 \\
696.0 \\
2.220\end{array}$ & $\begin{array}{l}116.0 \\
718.8 \\
2.274\end{array}$ & $\begin{array}{l}119.1 \\
742.0 \\
2.328\end{array}$ & $\begin{array}{l}122.2 \\
765.5 \\
2.382\end{array}$ \\
\hline$\epsilon 00$ & $\begin{array}{l}\text { D } \\
\text { S } \\
\mathrm{S}\end{array}$ & $\begin{array}{l}72.91 \\
54 \equiv .8 \\
1.792\end{array}$ & $\begin{array}{l}75.96 \\
564.3 \\
1.851\end{array}$ & $\begin{array}{l}78.94 \\
585.1 \\
1.909\end{array}$ & $\begin{array}{l}81.86 \\
601.2 \\
1.966\end{array}$ & $\begin{array}{l}84.72 \\
627.6 \\
2.023\end{array}$ & $\begin{array}{l}87.53 \\
649.4 \\
2.079\end{array}$ & $\begin{array}{l}90.30 \\
671.6 \\
2.135\end{array}$ & $\begin{array}{l}93.04 \\
694.1 \\
2.190\end{array}$ & $\begin{array}{l}95.75 \\
717.0 \\
2.245\end{array}$ & $\begin{array}{l}98.43 \\
740.3 \\
2.299\end{array}$ & $\begin{array}{l}101.1 \\
763.9 \\
2.353\end{array}$ \\
\hline 700 & $\begin{array}{l}y \\
y \\
s\end{array}$ & $\begin{array}{l}61.05 \\
54 C .4 \\
1.763\end{array}$ & $\begin{array}{l}63.80 \\
561.2 \\
1.823\end{array}$ & $\begin{array}{l}66.47 \\
582.2 \\
1.881\end{array}$ & $\begin{array}{l}69.06 \\
603.6 \\
1.939\end{array}$ & $\begin{array}{l}71.60 \\
625.2 \\
1.997\end{array}$ & $\begin{array}{l}74.08 \\
647.2 \\
2.053\end{array}$ & $\begin{array}{l}76.52 \\
669.5 \\
2.109\end{array}$ & $\begin{array}{l}78.93 \\
692.1 \\
2.165\end{array}$ & $\begin{array}{l}81.30 \\
715.2 \\
2.220\end{array}$ & $\begin{array}{l}83.65 \\
738.5 \\
2.274\end{array}$ & $\begin{array}{l}85.57 \\
762.3 \\
2.328\end{array}$ \\
\hline 800 & $\begin{array}{l}y \\
H \\
S\end{array}$ & $\begin{array}{l}52.10 \\
53 \epsilon .8 \\
1.736\end{array}$ & $\begin{array}{l}54.44 \\
558.0 \\
1.797\end{array}$ & $\begin{array}{l}57.08 \\
579.3 \\
1.856\end{array}$ & $\begin{array}{l}59.44 \\
600.9 \\
1.915\end{array}$ & $\begin{array}{l}61.74 \\
622.7 \\
1.973\end{array}$ & $\begin{array}{l}63.98 \\
644.9 \\
2.030\end{array}$ & $\begin{array}{l}66.18 \\
667.4 \\
2.086\end{array}$ & $\begin{array}{l}68.33 \\
690.2 \\
2.142\end{array}$ & $\begin{array}{l}70.46 \\
713.3 \\
2.197\end{array}$ & $\begin{array}{l}72.55 \\
736.8 \\
2.252\end{array}$ & $\begin{array}{l}74.62 \\
760.6 \\
2.307\end{array}$ \\
\hline 950 & $\begin{array}{l}7 \\
H \\
S\end{array}$ & $\begin{array}{l}45.09 \\
533.0 \\
1.711\end{array}$ & $\begin{array}{l}47.67 \\
554.6 \\
1.773\end{array}$ & $\begin{array}{l}49.74 \\
576.2 \\
1.833\end{array}$ & $\begin{array}{l}51.93 \\
598.1 \\
1.893\end{array}$ & $\begin{array}{l}54.05 \\
620.2 \\
1.951\end{array}$ & $\begin{array}{l}56.11 \\
642.5 \\
2.009\end{array}$ & $\begin{array}{l}58.12 \\
665.2 \\
2.065\end{array}$ & $\begin{array}{l}60.08 \\
688.1 \\
2.122\end{array}$ & $\begin{array}{l}62.02 \\
711.4 \\
2.177\end{array}$ & $\begin{array}{l}63.92 \\
735.0 \\
2.232\end{array}$ & $\begin{array}{l}65.80 \\
758.9 \\
2.287\end{array}$ \\
\hline 1030 & $\begin{array}{l}V \\
H \\
S\end{array}$ & $\begin{array}{l}39.41 \\
529.0 \\
1.6 B 7\end{array}$ & $\begin{array}{l}41 . \in 9 \\
551.0 \\
1.7 \leq 0\end{array}$ & $\begin{array}{l}43.84 \\
573.1 \\
1.812\end{array}$ & $\begin{array}{l}15.90 \\
595.2 \\
1.672\end{array}$ & $\begin{array}{l}47.88 \\
617.5 \\
1.931\end{array}$ & $\begin{array}{l}49.79 \\
640.1 \\
1.989\end{array}$ & $\begin{array}{l}51.66 \\
662.9 \\
2.046\end{array}$ & $\begin{array}{l}53.48 \\
686.1 \\
2.103\end{array}$ & $\begin{array}{l}55.26 \\
709.5 \\
2.159\end{array}$ & $\begin{array}{l}57.01 \\
733.2 \\
2.214\end{array}$ & $\begin{array}{l}58.73 \\
757.2 \\
2.269\end{array}$ \\
\hline
\end{tabular}


Table 12 (Continued)

Temperature range: 70 to $170^{\circ} \mathrm{C}$

\begin{tabular}{|c|c|c|c|c|c|c|c|c|c|c|c|c|c|}
\hline P & & T & 70 & 80 & 90 & 100 & 110 & 120 & 130 & 140 & 150 & 160 & 170 \\
\hline 1200 & $\begin{array}{l}\mathrm{T} \\
\mathrm{H} \\
\mathrm{s}\end{array}$ & & & $\begin{array}{l}32.88 \\
543.3 \\
1.707\end{array}$ & $\begin{array}{l}34.89 \\
566.3 \\
1.771\end{array}$ & $\begin{array}{l}36.72 \\
589.1 \\
1.831\end{array}$ & $\begin{array}{l}38.57 \\
612.0 \\
1.894\end{array}$ & $\begin{array}{l}40.28 \\
635.1 \\
1.953\end{array}$ & $\begin{array}{l}41.93 \\
658.3 \\
2.012\end{array}$ & $\begin{array}{l}43.54 \\
681.8 \\
2.069\end{array}$ & $\begin{array}{l}45.10 \\
705.5 \\
2.126\end{array}$ & $\begin{array}{l}\begin{array}{r}46.62 \\
729.5 \\
2182\end{array}\end{array}$ & $\begin{array}{l}48.12 \\
753.8 \\
2.238\end{array}$ \\
\hline 1400 & $\begin{array}{l}\text { D } \\
\text { H } \\
\text { s }\end{array}$ & & & & $\begin{array}{l}28.36 \\
558.7 \\
1.733\end{array}$ & $\begin{array}{l}30.15 \\
582.5 \\
1.793\end{array}$ & $\begin{array}{l}31.84 \\
606.2 \\
1.860\end{array}$ & $\begin{array}{r}33.43 \\
629.8 \\
1.921\end{array}$ & $\begin{array}{l}34.95 \\
653.5 \\
1.981\end{array}$ & $\begin{array}{l}36.40 \\
677.3 \\
2.039\end{array}$ & $\begin{array}{l}37.81 \\
701.4 \\
2.097\end{array}$ & $\begin{array}{l}39.18 \\
725.7 \\
2.154\end{array}$ & $\begin{array}{l}40.52 \\
750.2 \\
2.210\end{array}$ \\
\hline 1600 & $\begin{array}{l}7 \\
H \\
5\end{array}$ & & & & $\begin{array}{l}23.28 \\
550.1 \\
1.695\end{array}$ & $\begin{array}{l}25.09 \\
575.2 \\
1.764\end{array}$ & $\begin{array}{l}26.72 \\
599.8 \\
1.829\end{array}$ & $\begin{array}{l}28.24 \\
624.1 \\
1.891\end{array}$ & $\begin{array}{l}29.67 \\
648.4 \\
1.952\end{array}$ & $\begin{array}{l}31.02 \\
672.7 \\
2.012\end{array}$ & $\begin{array}{l}32.33 \\
697.1 \\
2.070\end{array}$ & $\begin{array}{l}33.59 \\
721.7 \\
2.128\end{array}$ & $\begin{array}{l}34.81 \\
706.6 \\
2.184\end{array}$ \\
\hline 1800 & $\begin{array}{l}\text { H } \\
\text { H } \\
S\end{array}$ & & & & & $\begin{array}{l}21.00 \\
566.9 \\
1.720\end{array}$ & $\begin{array}{l}22.65 \\
592.8 \\
1.797\end{array}$ & $\begin{array}{l}24.14 \\
618.0 \\
1.862\end{array}$ & $\begin{array}{l}25.51 \\
692.9 \\
1.925\end{array}$ & $\begin{array}{l}26.81 \\
667.8 \\
1.986\end{array}$ & $\begin{array}{l}28.04 \\
692.7 \\
2.045\end{array}$ & $\begin{array}{l}29.21 \\
717.6 \\
2.104\end{array}$ & $\begin{array}{l}30.35 \\
742.8 \\
2.161\end{array}$ \\
\hline 2000 & $\begin{array}{l}\text { Y } \\
\text { H } \\
\text { S }\end{array}$ & & & & & & $\begin{array}{l}19.28 \\
584.9 \\
1.766\end{array}$ & $\begin{array}{l}20.79 \\
611.3 \\
1.834\end{array}$ & $\begin{array}{l}22.15 \\
637.1 \\
1.899\end{array}$ & $\begin{array}{l}23.40 \\
662.6 \\
1.961\end{array}$ & $\begin{array}{l}24.58 \\
688.0 \\
2.022\end{array}$ & $\begin{array}{l}25.70 \\
713.4 \\
2.081\end{array}$ & $\begin{array}{l}26.77 \\
738.9 \\
2.139\end{array}$ \\
\hline 2100 & $\begin{array}{l}\mathrm{y} \\
\mathrm{H} \\
\mathrm{S}\end{array}$ & & & & & & $\begin{array}{l}17.79 \\
580.5 \\
1.750\end{array}$ & $\begin{array}{l}19.32 \\
607.7 \\
1.820\end{array}$ & $\begin{array}{l}20.68 \\
634.0 \\
1.886\end{array}$ & $\begin{array}{l}21.93 \\
659.9 \\
1.949\end{array}$ & $\begin{array}{l}23.09 \\
685.6 \\
2.011\end{array}$ & $\begin{array}{l}24.19 \\
711.2 \\
2070\end{array}$ & $\begin{array}{l}25.23 \\
736.9 \\
2.129\end{array}$ \\
\hline 2200 & $\begin{array}{l}\mathrm{y} \\
\mathrm{y} \\
\mathrm{s}\end{array}$ & & & & & & $\begin{array}{l}16.38 \\
575.7 \\
1.733\end{array}$ & $\begin{array}{l}17.97 \\
603.9 \\
1.805\end{array}$ & $\begin{array}{l}19.34 \\
630.8 \\
1.873\end{array}$ & $\begin{array}{l}20.58 \\
657.1 \\
1.937\end{array}$ & $\begin{array}{l}21.72 \\
683.1 \\
1.999\end{array}$ & $\begin{array}{l}22.80 \\
708.9 \\
2.060\end{array}$ & $\begin{array}{l}23.83 \\
736.8 \\
2.119\end{array}$ \\
\hline 2300 & $\begin{array}{l}8 \\
\text { H } \\
s\end{array}$ & & & & & & $\begin{array}{l}15.04 \\
570.4 \\
1.715\end{array}$ & $\begin{array}{l}16.70 \\
599.8 \\
1.791\end{array}$ & $\begin{array}{l}18.09 \\
627.4 \\
1.860\end{array}$ & $\begin{array}{l}19.33 \\
654.2 \\
1.925\end{array}$ & $\begin{array}{l}20.47 \\
680.5 \\
1.988\end{array}$ & $\begin{array}{l}21.54 \\
706.6 \\
2.049\end{array}$ & $\begin{array}{l}22.55 \\
732.7 \\
2.109\end{array}$ \\
\hline 2400 & $\begin{array}{l}\text { Y } \\
\text { H } \\
5\end{array}$ & & & & & 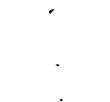 & & $\begin{array}{l}15.51 \\
595.4 \\
1.775\end{array}$ & $\begin{array}{l}16.94 \\
623.9 \\
1.847\end{array}$ & $\begin{array}{l}18.19 \\
65.1 .2 \\
1.914\end{array}$ & $\begin{array}{l}19.32 \\
677.9 \\
1.977\end{array}$ & $\begin{array}{l}20.37 \\
704.3 \\
2.039\end{array}$ & $\begin{array}{l}21.37 \\
730.6 \\
2.099\end{array}$ \\
\hline 2500 & $\begin{array}{l}\text { D } \\
\text { H } \\
\text { s }\end{array}$ & & & & & & & $\begin{array}{l}14.37 \\
590.7 \\
1.759\end{array}$ & $\begin{array}{l}15.85 \\
620.1 \\
1.833\end{array}$ & $\begin{array}{l}17.12 \\
648.0 \\
1.902\end{array}$ & $\begin{array}{l}18.25 \\
675.1 \\
1.967\end{array}$ & $\begin{array}{l}19.30 \\
701.9 \\
2.029\end{array}$ & $\begin{array}{l}20.28 \\
728.4 \\
2.090\end{array}$ \\
\hline 2600 & $\begin{array}{l}\mathrm{V} \\
\mathrm{H} \\
\mathrm{s}\end{array}$ & & & & & & & $\begin{array}{l}13.28 \\
585.4 \\
1.792\end{array}$ & $\begin{array}{l}14.83 \\
616: 1 \\
1.820\end{array}$ & $\begin{array}{l}16.12 \\
644.7 \\
1.890\end{array}$ & $\begin{array}{l}17.26 \\
672.3 \\
1.956\end{array}$ & $\begin{array}{l}18.30 \\
699.4 \\
2.019\end{array}$ & $\begin{array}{l}19.27 \\
726.2 \\
2.080\end{array}$ \\
\hline 2700 & $\begin{array}{l}\text { D } \\
\text { H } \\
S\end{array}$ & & & & & & & $\begin{array}{l}12.20 \\
579.3 \\
1.724\end{array}$ & $\begin{array}{l}13.86 \\
611.9 \\
1.806\end{array}$ & $\begin{array}{l}15.18 \\
641.3 \\
1.878\end{array}$ & $\begin{array}{l}16.33 \\
669.4 \\
1.945\end{array}$ & $\begin{array}{l}17.37 \\
696.8 \\
2.009\end{array}$ & $\begin{array}{l}18.33 \\
723.9 \\
2.071\end{array}$ \\
\hline 2800 & $\begin{array}{l}\text { D } \\
\text { H } \\
S\end{array}$ & & & & & & & $\begin{array}{l}11.11 \\
572.2 \\
1.703\end{array}$ & $\begin{array}{l}12.93 \\
607.2 \\
1.791\end{array}$ & $\begin{array}{l}14.30 \\
637.7 \\
1.865\end{array}$ & $\begin{array}{l}15.46 \\
666.4 \\
1.934\end{array}$ & $\begin{array}{l}16.50 \\
694.2 \\
1.999\end{array}$ & $\begin{array}{l}17.46 \\
721.6 \\
2.062\end{array}$ \\
\hline
\end{tabular}


Table 12 (Continued)

Temperature range: 70 to $170^{\circ} \mathrm{C}$

\begin{tabular}{|c|c|c|c|c|c|c|c|c|c|c|c|c|c|}
\hline $\mathbf{P}$ & & I & 70 & 30 & 90 & 100 & 110 & 120 & 130 & 140 & 150 & 160 & 170 \\
\hline 2500 & i & & & & & & & & $\begin{array}{l}12.03 \\
602.1 \\
1.775\end{array}$ & $\begin{array}{l}13.46 \\
633.9 \\
1.853\end{array}$ & $\begin{array}{l}14.64 \\
663.3 \\
1.923\end{array}$ & $\begin{array}{l}15.69 \\
69.5 \\
1.989\end{array}$ & $\begin{array}{l}16.64 \\
719.2 \\
2.052\end{array}$ \\
\hline 3000 & $\begin{array}{l}\mathbf{r} \\
\mathrm{H} \\
\mathrm{S}\end{array}$ & & & & & & & & $\begin{array}{l}11.14 \\
596.4 \\
1.758\end{array}$ & $\begin{array}{l}12.66 \\
629.8 \\
1.800\end{array}$ & $\begin{array}{l}13.87 \\
660.0 \\
1.912\end{array}$ & $\begin{array}{l}14.92 \\
688.8 \\
1.979\end{array}$ & $\begin{array}{l}15.87 \\
716.8 \\
2.043\end{array}$ \\
\hline 3200 & $\begin{array}{l}\mathrm{T} \\
\mathrm{E} \\
\mathbf{s}\end{array}$ & & & & & & ' & & $\begin{array}{l}10.25 \\
589.9 \\
1.739\end{array}$ & $\begin{array}{l}11.89 \\
625.4 \\
1.826\end{array}$ & $\begin{array}{l}13.11 \\
656.6 \\
1.901\end{array}$ & $\begin{array}{l}14.20 \\
685.9 \\
1.969\end{array}$ & $\begin{array}{l}15.15 \\
714.3 \\
2.034\end{array}$ \\
\hline 3200 & $\begin{array}{l}\mathrm{V} \\
\mathrm{H} \\
\mathrm{S}\end{array}$ & & & & & & & & $\begin{array}{l}9.322 \\
581.9 \\
1.717\end{array}$ & $\begin{array}{l}11.14 \\
620.7 \\
1.812\end{array}$ & $\begin{array}{l}12.44 \\
653.0 \\
1.889\end{array}$ & $\begin{array}{l}13.52 \\
682.9 \\
1.959\end{array}$ & $\begin{array}{l}14.47 \\
711.8 \\
2.025\end{array}$ \\
\hline 3300 & $\begin{array}{l}y \\
y \\
s\end{array}$ & & & & & & & & $\begin{array}{l}8.276 \\
571.3 \\
1.688\end{array}$ & $\begin{array}{l}10.42 \\
615.6 \\
1.797\end{array}$ & $\begin{array}{l}11.77 \\
649.2 \\
1.877\end{array}$ & $\begin{array}{l}12.87 \\
679.9 \\
1.949\end{array}$ & $\begin{array}{l}13.83 \\
709.2 \\
2.016\end{array}$ \\
\hline 3400 & $\begin{array}{l}\mathrm{V} \\
\mathrm{B} \\
\mathrm{S}\end{array}$ & & & & & & & & & $\begin{array}{l}9.696 \\
609.8 \\
1.781\end{array}$ & $\begin{array}{l}11.13 \\
645.2 \\
1.865\end{array}$ & $\begin{array}{l}12.25 \\
676.7 \\
1.939\end{array}$ & $\begin{array}{l}\begin{array}{l}13.22 \\
706.5 \\
2.007\end{array}\end{array}$ \\
\hline 3530 & $\begin{array}{l}r \\
H \\
S\end{array}$ & & & & & & & & & $\begin{array}{l}8.971 \\
603.3 \\
1.762\end{array}$ & $\begin{array}{l}10.51 \\
640.9 \\
1.853\end{array}$ & $\begin{array}{l}11.67 \\
673.4 \\
1.928\end{array}$ & $\begin{array}{l}12.65 \\
703.7 \\
1.998\end{array}$ \\
\hline 36000 & $\begin{array}{l}\mathrm{T} \\
\mathrm{H} \\
\mathrm{S}\end{array}$ & & & & & & & & & $\begin{array}{l}8.225 \\
595.6 \\
1.742\end{array}$ & $\begin{array}{l}9.911 \\
636.4 \\
1.839\end{array}$ & $\begin{array}{l}11.11 \\
669.9 \\
1.918\end{array}$ & $\begin{array}{l}12.10 \\
700-9 \\
1.988\end{array}$ \\
\hline ביס & $\begin{array}{l}\nabla \\
\text { H } \\
S\end{array}$ & & & & & & & & & $\begin{array}{l}7.426 \\
586.0 \\
1.717\end{array}$ & $\begin{array}{l}9.325 \\
631.4 \\
1.825\end{array}$ & $\begin{array}{l}10.57 \\
666.3 \\
1.907\end{array}$ & $\begin{array}{l}11.58 \\
697.9 \\
1.979\end{array}$ \\
\hline 3800 & $\begin{array}{l}\nabla \\
H \\
S\end{array}$ & & & & & & & & & $\begin{array}{l}6.507 \\
572.8 \\
1.683\end{array}$ & $\begin{array}{l}8.751 \\
626.1 \\
1.811\end{array}$ & $\begin{array}{l}10.05 \\
662.5 \\
1.899\end{array}$ & $\begin{array}{l}11.08 \\
694.9 \\
1.970\end{array}$ \\
\hline 3900 & $\begin{array}{l}y \\
y \\
\text { s }\end{array}$ & & & & & & & & & $\begin{array}{l}5.334 \\
551.7 \\
1.630\end{array}$ & $\begin{array}{l}8.182 \\
620.2 \\
1.795\end{array}$ & $\begin{array}{l}9.549 \\
658.5 \\
1.884\end{array}$ & $\begin{array}{l}10.60 \\
691.7 \\
1.960\end{array}$ \\
\hline 4000 & $\begin{array}{l}Y \\
H \\
S\end{array}$ & & & & r & & $\cdots$ & & & $\begin{array}{l}4.203 \\
524.3 \\
1.563\end{array}$ & $\begin{array}{l}7.616 \\
613.6 \\
1.777\end{array}$ & $\begin{array}{l}9.066 \\
654.3 \\
1.872\end{array}$ & $\begin{array}{l}10.18 \\
688.5 \\
1.951\end{array}$ \\
\hline 4100 & $\begin{array}{l}r \\
H \\
S\end{array}$ & & & & & & & & & $\begin{array}{l}3.667 \\
507.5 \\
1.522\end{array}$ & $\begin{array}{l}7.049 \\
606.3 \\
1.758\end{array}$ & $\begin{array}{l}8.598 \\
649.9 \\
1.860\end{array}$ & $\begin{array}{l}9.703 \\
685.1 \\
1.991\end{array}$ \\
\hline
\end{tabular}


Table 12 (Continued)

Temperature range: 70 to $170^{\circ} \mathrm{C}$

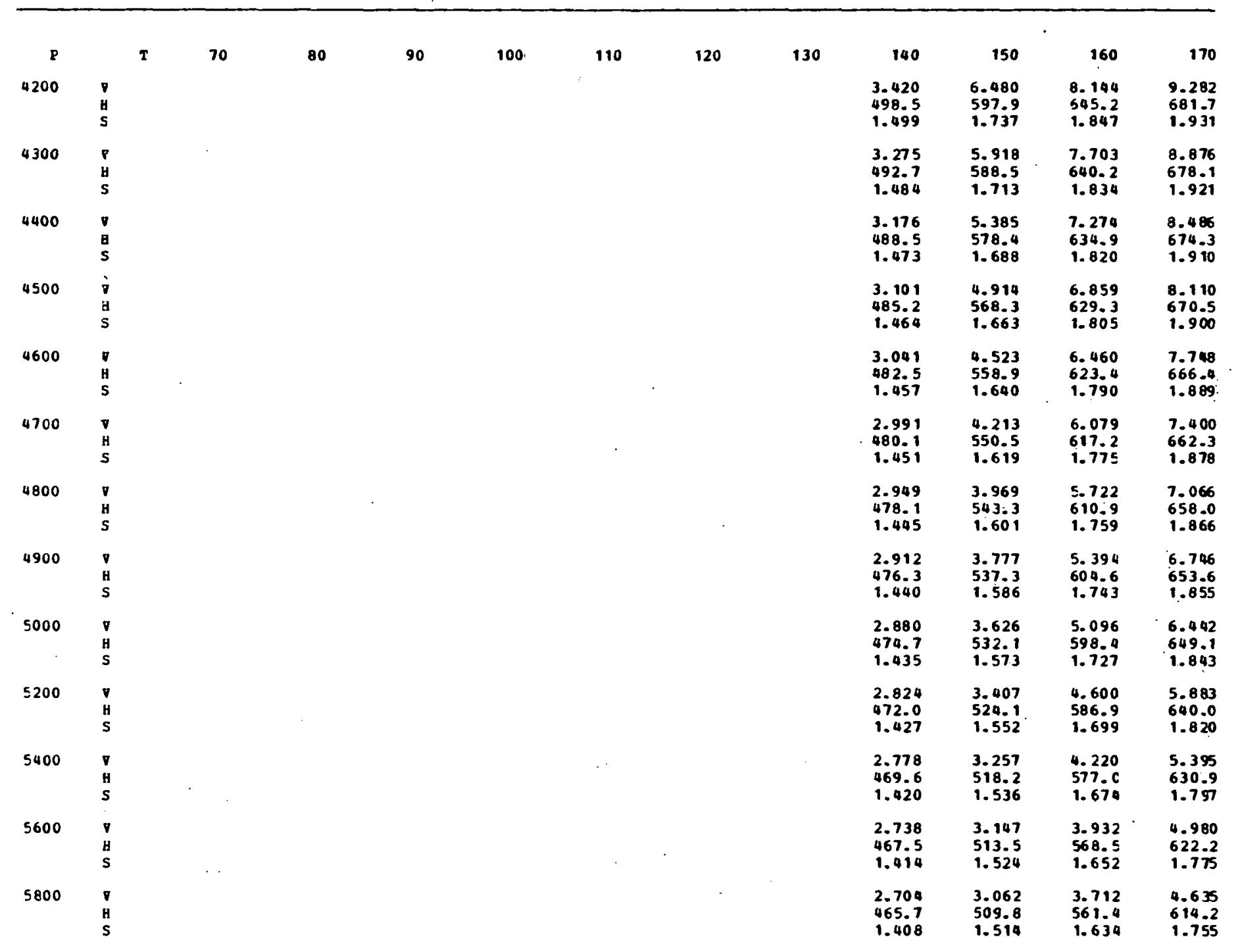


Table 12 (Continued)

Temperature range: 70 to $170^{\circ} \mathrm{C}$

\begin{tabular}{|c|c|c|c|c|c|c|c|c|c|c|c|c|c|}
\hline P & & $T$ & 70 & 80 & 90 & 100 & 110 & 120 & 130 & 140 & 150 & 160 & 170 \\
\hline 6000 & $\begin{array}{l}\dot{y} \\
\mathrm{H} \\
\mathrm{s}\end{array}$ & & & & & & & & & $\begin{array}{l}2.674 \\
464.7 \\
1.403\end{array}$ & $\begin{array}{l}2.993 \\
506.7 \\
1.505\end{array}$ & $\begin{array}{l}3.542 \\
555.5 \\
1.619\end{array}$ & $\begin{array}{l}4.350 \\
607.0 \\
1.736\end{array}$ \\
\hline 6200 & $\begin{array}{l}\nabla \\
H \\
S\end{array}$ & & & & & & & & & $\begin{array}{l}2.646 \\
462.6 \\
1.398\end{array}$ & $\begin{array}{l}2.936 \\
504.0 \\
1.497\end{array}$ & $\begin{array}{l}3.40 \varepsilon \\
550.6 \\
1-606\end{array}$ & $\begin{array}{l}4.116 \\
600.5 \\
1.720\end{array}$ \\
\hline 6700 & $\begin{array}{l}\text { V } \\
H \\
S\end{array}$ & & & & & & & & & $\begin{array}{l}2.622 \\
461.3 \\
1.394\end{array}$ & $\begin{array}{l}2.887 \\
501.7 \\
1.490\end{array}$ & $\begin{array}{l}3.301 \\
546.4 \\
1.599\end{array}$ & $\begin{array}{l}3.921 \\
594.7 \\
1.705\end{array}$ \\
\hline 6500 & $\begin{array}{l}\nabla \\
H \\
\text { S }\end{array}$ & & & & & & & & & $\begin{array}{l}2.599 \\
460.1 \\
1.389\end{array}$ & $\begin{array}{l}2.944 \\
999.7 \\
1.484\end{array}$ & $\begin{array}{l}3.212 \\
542.9 \\
1.58 \leq\end{array}$ & $\begin{array}{l}3.760 \\
589.6 \\
1.692\end{array}$ \\
\hline 6300 & $\begin{array}{l}\text { I } \\
\text { H } \\
\text { S }\end{array}$ & & & & & & & & & $\begin{array}{l}2.578 \\
458.9 \\
1.385\end{array}$ & $\begin{array}{l}2.806 \\
497.9 \\
1.479\end{array}$ & $\begin{array}{l}3.13 E \\
539.8 \\
1.57 \epsilon\end{array}$ & $\begin{array}{l}3.624 \\
585.1 \\
1.680\end{array}$ \\
\hline 7.300 & $\begin{array}{l}\text { V } \\
H \\
\text { S }\end{array}$ & & & & & & & & & $\begin{array}{l}2.559 \\
457.9 \\
1.382\end{array}$ & $\begin{array}{l}2.773 \\
496.2 \\
1.473\end{array}$ & $\begin{array}{l}3.075 \\
537.1 \\
1.569\end{array}$ & $\begin{array}{l}3.510 \\
581.1 \\
1.669\end{array}$ \\
\hline 7500 & $\begin{array}{l}V \\
H \\
S\end{array}$ & & & & & & & - & & $\begin{array}{l}2.517 \\
455.6 \\
1.373\end{array}$ & $\begin{array}{l}2.702 \\
492.7 \\
1.462 .\end{array}$ & $\begin{array}{l}2.950 \\
531.6 \\
1.555\end{array}$ & $\begin{array}{l}3.291 \\
572.9 \\
1.647\end{array}$ \\
\hline 8000 & $\begin{array}{l}\text { V } \\
\text { H } \\
\text { S }\end{array}$ & & & & & & & & & $\begin{array}{l}2.481 \\
453.7 \\
1.365\end{array}$ & $\begin{array}{l}2.646 \\
489.9 \\
1.452\end{array}$ & $\begin{array}{l}2.857 \\
527.4 \\
1.539\end{array}$ & $\begin{array}{l}3.135 \\
556.7 \\
1.629\end{array}$ \\
\hline $8: 00$ & $\begin{array}{l}V \\
H \\
\text { s. }\end{array}$ & & & & & & & & & $\begin{array}{l}2.450 \\
.52 .1 \\
1.358\end{array}$ & $\begin{array}{l}2.598 \\
487.5 \\
1.443\end{array}$ & $\begin{array}{l}2.783 \\
523.9 \\
1.528\end{array}$ & $\begin{array}{l}3.019 \\
561.8 \\
1.615\end{array}$ \\
\hline 9000 & $\begin{array}{l}\mathbf{V} \\
\text { H } \\
\text { S }\end{array}$ & & & & & & & & & $\begin{array}{l}2.422 \\
450.6 \\
1.352\end{array}$ & $\begin{array}{l}2.558 \\
485.5 \\
1.435\end{array}$ & $\begin{array}{l}2.723 \\
521.1 \\
1.58 .9\end{array}$ & $\begin{array}{l}2.927 \\
557.8 \\
1.602\end{array}$ \\
\hline $10<00$ & $\begin{array}{l}\mathbf{y} \\
\text { H } \\
\text { s }\end{array}$ & & & & & & & $\ldots$ & & $\begin{array}{l}2.375 \\
448.3 \\
1.341\end{array}$ & $\begin{array}{l}2.491 \\
482.2 \\
1.422\end{array}$ & $\begin{array}{l}2.628 \\
516.7 \\
1.502\end{array}$ & $\begin{array}{l}2.790 \\
551.8 \\
1.582\end{array}$ \\
\hline 11000 & $\begin{array}{l}\nabla \\
H \\
S\end{array}$ & & & & & & & & & $\begin{array}{l}2.337 \\
446.6 \\
1.331\end{array}$ & $\begin{array}{l}2.438 \\
479.7 \\
1.410\end{array}$ & $\begin{array}{l}2.556 \\
513.3 \\
1.488\end{array}$ & $\begin{array}{l}2.692 \\
547.4 \\
1.566\end{array}$ \\
\hline 12000 & $\begin{array}{l}V \\
H \\
s\end{array}$ & & & & & & & & & $\begin{array}{l}2.304 \\
445.2 \\
1.322\end{array}$ & $\begin{array}{l}2.395 \\
477.8 \\
1.000\end{array}$ & $\begin{array}{l}2.098 \\
510.8 \\
1.477\end{array}$ & $\begin{array}{l}2.615 \\
544.1 \\
1.553\end{array}$ \\
\hline
\end{tabular}


Table 12 (Continued)

Temperature range: 70 to $170^{\circ} \mathrm{C}$

\begin{tabular}{|c|c|c|c|c|c|c|c|c|c|c|c|c|}
\hline $\mathbf{P}$ & $I$ & 70 & 80 & 90 & 100 & 110 & 120 & 130 & 140 & 150 & 160 & 170 \\
\hline 13000 & $\begin{array}{l}8 . \\
8 \\
5\end{array}$ & & & & & & & & $\begin{array}{l}2.276 \\
444.2 \\
1.314\end{array}$ & $\begin{array}{l}2.358 \\
976.3 \\
1.391\end{array}$ & $\begin{array}{l}2.450 \\
508.7 \\
1.466\end{array}$ & $\begin{array}{l}2.550 \\
541.5 \\
1.541\end{array}$ \\
\hline 10000 & $\begin{array}{l}\text { V } \\
\text { a } \\
\text { s }\end{array}$ & & & & & & & & $\begin{array}{l}2.251 \\
443.5 \\
1.307\end{array}$ & $\begin{array}{l}2.326 \\
475.1 \\
1.382\end{array}$ & $\begin{array}{l}2.410 \\
507.1 \\
1.457\end{array}$ & $\begin{array}{l}2.503 \\
539.4 \\
1.531\end{array}$ \\
\hline 15000 & $\begin{array}{l}7 \\
\text { H } \\
5\end{array}$ & & & & & & & & $\begin{array}{l}2.229 \\
443.0 \\
1.300\end{array}$ & $\begin{array}{l}2.298 \\
474.2 \\
1.375\end{array}$ & $\begin{array}{l}2.375 \\
505.9 \\
1.049\end{array}$ & $\begin{array}{l}2.460 \\
537.8 \\
1.521\end{array}$ \\
\hline 16000 & $\begin{array}{l}\text { V } \\
\text { H } \\
\text { S }\end{array}$ & & & & & & & & $\begin{array}{l}2.210 \\
442.6 \\
1.294\end{array}$ & $\begin{array}{l}2.273 \\
473.5 \\
1.368\end{array}$ & $\begin{array}{l}2.344 \\
504.9 \\
1.491\end{array}$ & $\begin{array}{r}2.422 \\
536.5 \\
1.513\end{array}$ \\
\hline 17000 & $\begin{array}{l}\text { V } \\
\text { H } \\
\text { S }\end{array}$ & & & & & & & & $\begin{array}{l}2.192 \\
442.5 \\
1.288\end{array}$ & $\begin{array}{l}2.251 \\
473.0 \\
1.361\end{array}$ & $\begin{array}{l}2.317 \\
504.1 \\
1.434\end{array}$ & $\begin{array}{l}2.389 \\
535.4 \\
1.505\end{array}$ \\
\hline 18000 & $\begin{array}{l}y \\
y \\
\text { s }\end{array}$ & & & & & & & & $\begin{array}{l}2.175 \\
442.4 \\
1.283\end{array}$ & $\begin{array}{l}2.231 \\
472.7 \\
1.355\end{array}$ & $\begin{array}{l}2.293 \\
503.5 \\
1.427\end{array}$ & $\begin{array}{l}2.360 \\
53.6 \\
1.498\end{array}$ \\
\hline 19000 & $\begin{array}{l}\text { D } \\
\text { H } \\
\text { s }\end{array}$ & & & & & ' & & & $\begin{array}{l}2.160 \\
442.5 \\
1.278\end{array}$ & $\begin{array}{l}2.213 \\
972.5 \\
1.349\end{array}$ & $\begin{array}{r}2.270 \\
503.1 \\
1.421\end{array}$ & $\begin{array}{l}2.333 \\
534.0 \\
1.491\end{array}$ \\
\hline 20000 & $\begin{array}{l}\mathbf{y} \\
\text { ü } \\
\text { s }\end{array}$ & & & & & & & & $\begin{array}{l}2.147 \\
42.7 \\
1.273\end{array}$ & $\begin{array}{r}2.196 \\
472.5 \\
1.344\end{array}$ & $\begin{array}{l}2.250 \\
502.8 \\
1.415\end{array}$ & $\begin{array}{l}2.309 \\
53.5 \\
1.485\end{array}$ \\
\hline 21000 & $\begin{array}{l}\mathbf{V} \\
\mathbf{H} \\
\mathbf{S}\end{array}$ & & & & 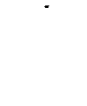 & . & & & $\begin{array}{l}2.134 \\
443.0 \\
1.268\end{array}$ & $\begin{array}{l}2.180 \\
472.5 \\
1.339\end{array}$ & $\begin{array}{l}2.232 \\
502.7 \\
1.409\end{array}$ & $\begin{array}{r}2.288 \\
.533 .2 \\
1.479\end{array}$ \\
\hline 22000 & $\begin{array}{l}J \\
\text { H } \\
\text { S }\end{array}$ & $\therefore$ & & & & & & & $\begin{array}{l}2.122 \\
443.4 \\
1.264\end{array}$ & $\begin{array}{l}2.166 \\
47.6 \\
1.334\end{array}$ & $\begin{array}{r}2.215 \\
502.6 \\
1.404\end{array}$ & $\begin{array}{l}2.268 \\
533.0 \\
1.473\end{array}$ \\
\hline 23000 & $\begin{array}{l}\text { V } \\
\text { H } \\
\text { S }\end{array}$ & & & & . : & & & & $\begin{array}{l}2.110 \\
443.9 \\
1.260\end{array}$ & $\begin{array}{l}2.152 \\
47.29 \\
1.330\end{array}$ & $\begin{array}{l}2.199 \\
502 . t \\
1.399\end{array}$ & $\begin{array}{l}2.249 \\
532.9 \\
1.468\end{array}$ \\
\hline 24000 & $\begin{array}{l}y \\
\text { H } \\
\text { s }\end{array}$ & & & & & & & & $\begin{array}{l}2.100 \\
444.4 \\
1.256\end{array}$ & $\begin{array}{l}2.140 \\
473.2 \\
1.325\end{array}$ & $\begin{array}{l}2.184 \\
552.8 \\
1.394\end{array}$ & $\begin{array}{l}2.232 \\
532.9 \\
1.463\end{array}$ \\
\hline 25000 & $\begin{array}{l}V \\
H \\
\text { S }\end{array}$ & & & & . & & & & $\begin{array}{l}2.090 \\
445.0 \\
1.253\end{array}$ & $\begin{array}{l}2.128 \\
473.5 \\
1.321\end{array}$ & $\begin{array}{l}2.170 \\
503.0 \\
1.390\end{array}$ & $\begin{array}{l}2.217 \\
532.9 \\
1.458\end{array}$ \\
\hline
\end{tabular}


Table 12 (Continued)

Temperature range: 180 to $280^{\circ} \mathrm{C}$

\begin{tabular}{|c|c|c|c|c|c|c|c|c|c|c|c|c|}
\hline $\mathbf{P}$ & & 180 & 190 & 200 & 210 & 220 & 230 & 240 & 250 & 260 & 270 & 280 \\
\hline 5 & $\begin{array}{l}\text { H } \\
H \\
\text { S }\end{array}$ & $\begin{array}{r}12960 \\
796.7 \\
3.105\end{array}$ & $\begin{array}{r}13250 . \\
820.6 \\
3.157\end{array}$ & $\begin{array}{r}13530 . \\
844.9 \\
3.209\end{array}$ & $\begin{array}{r}13820 \\
869.7 \\
3.261\end{array}$ & $\begin{array}{r}14110 . \\
894.8 \\
3.313\end{array}$ & $\begin{array}{r}14390 \\
920.3 \\
3.364\end{array}$ & $\begin{array}{r}14680 \\
946.2 \\
3.415\end{array}$ & $\begin{array}{r}94960 . \\
972.5 \\
3.465\end{array}$ & $\begin{array}{r}15250 \\
999.1 \\
3.516\end{array}$ & $\begin{array}{c}15540 \\
1026 \\
3.56 \epsilon\end{array}$ & $\begin{array}{r}15820 . \\
1054 . \\
3.616\end{array}$ \\
\hline 50 & $\begin{array}{l}y \\
4 \\
5\end{array}$ & $\begin{array}{l}1290 . \\
796.0 \\
2.775\end{array}$ & $\begin{array}{l}1320 \\
820.0 \\
2.827\end{array}$ & $\begin{array}{l}1350 . \\
844.4 \\
2.879\end{array}$ & $\begin{array}{l}1380 \\
869.1 \\
2.931\end{array}$ & $\begin{array}{l}1410 . \\
894.3 \\
2.983\end{array}$ & $\begin{array}{l}1440 . \\
919.8 \\
3.034\end{array}$ & $\begin{array}{l}1470 . \\
945.7 \\
3.085\end{array}$ & $\begin{array}{l}1490 . \\
972.0 \\
3.136\end{array}$ & $\begin{array}{l}1520 \\
998.7 \\
3.186\end{array}$ & $\begin{array}{l}1550 . \\
1026 . \\
3.236\end{array}$ & $\begin{array}{l}1580 . \\
1053 . \\
3.286\end{array}$ \\
\hline 100 & $\begin{array}{l}y \\
5\end{array}$ & $\begin{array}{l}644.0 \\
795.3 \\
2.675\end{array}$ & $\begin{array}{l}658.5 \\
819.3 \\
2.727\end{array}$ & $\begin{array}{l}673.0 \\
843.7 \\
2.779\end{array}$ & $\begin{array}{l}687.5 \\
868.5 \\
2.831\end{array}$ & $\begin{array}{l}702.0 \\
893.7 \\
2.883\end{array}$ & $\begin{array}{l}716.5 \\
919.2 \\
2.934\end{array}$ & $\begin{array}{l}730.9 \\
945.2 \\
2.985\end{array}$ & $\begin{array}{l}745.4 \\
971.5 \\
3.036\end{array}$ & $\begin{array}{l}759.8 \\
998.2 \\
3.086\end{array}$ & $\begin{array}{l}774.3 \\
1025 . \\
3.136\end{array}$ & $\begin{array}{l}788.7 \\
1053 . \\
3.186\end{array}$ \\
\hline 101.33 & $\begin{array}{l}7 \\
8 \\
5\end{array}$ & $\begin{array}{l}635-5 \\
795-3 \\
2.673\end{array}$ & $\begin{array}{l}649.8 \\
819.3 \\
2.725\end{array}$ & $\begin{array}{l}664.1 \\
843.7 \\
2.777\end{array}$ & $\begin{array}{l}678.5 \\
868.5 \\
2.829\end{array}$ & $\begin{array}{l}692.8 \\
893.7 \\
2.881\end{array}$ & $\begin{array}{l}707.0 \\
919.2 \\
2.932\end{array}$ & $\begin{array}{l}721.3 \\
945.2 \\
2.983\end{array}$ & $\begin{array}{l}735.6 \\
971.5 \\
3.034\end{array}$ & $\begin{array}{l}749.8 \\
998.2 \\
3.084\end{array}$ & $\begin{array}{l}764.1 \\
1025 . \\
3.135\end{array}$ & $\begin{array}{l}778.3 \\
1053 . \\
3.185\end{array}$ \\
\hline 200 & $\begin{array}{l}\text { y } \\
\text { B } \\
\text { S }\end{array}$ & $\begin{array}{l}319.9 \\
793.9 \\
2.573\end{array}$ & $\begin{array}{l}327.2 \\
817.9 \\
2.626\end{array}$ & $\begin{array}{l}334.6 \\
842.4 \\
2.678\end{array}$ & $\begin{array}{l}341.9 \\
867.2 \\
2.730\end{array}$ & $\begin{array}{l}349.3 \\
892.5 \\
2.782\end{array}$ & $\begin{array}{l}356.6 \\
918.1 \\
2.833\end{array}$ & $\begin{array}{l}363.9 \\
944.1 \\
2.884\end{array}$ & $\begin{array}{l}371.2 \\
970.4 \\
2.93 .5\end{array}$ & $\begin{array}{l}378.5 \\
997.1 \\
2.986\end{array}$ & $\begin{array}{l}385.8 \\
1024 . \\
3.036\end{array}$ & $\begin{array}{l}393.1 \\
1052 . \\
3.086\end{array}$ \\
\hline 300 & $\begin{array}{l}P \\
\text { H } \\
S\end{array}$ & $\begin{array}{l}211.8 \\
792-4 \\
2.513\end{array}$ & $\begin{array}{l}216.8 \\
816.5 \\
2.566\end{array}$ & $\begin{array}{l}229.8 \\
841.1 \\
2.518\end{array}$ & $\begin{array}{l}226.7 \\
866.0 \\
2.670\end{array}$ & $\begin{array}{l}231.7 \\
891.3 \\
2.722\end{array}$ & $\begin{array}{l}236.6 \\
516.9 \\
2.773\end{array}$ & $\begin{array}{l}241.6 \\
943.0 \\
2.825\end{array}$ & $\begin{array}{l}246.5 \\
969.4 \\
2.876\end{array}$ & $\begin{array}{l}251.4 \\
996.1 \\
2.926\end{array}$ & $\begin{array}{l}256.3 \\
1023 \\
2.977\end{array}$ & $\begin{array}{l}261.2 \\
1051 . \\
3.027\end{array}$ \\
\hline 400 & $\begin{array}{l}\text { P } \\
\text { H } \\
\text { S }\end{array}$ & $\begin{array}{l}157.8 \\
796.9 \\
2.469\end{array}$ & $\begin{array}{l}161.6 \\
815.1 \\
2.522\end{array}$ & $\begin{array}{l}165.4 \\
839.7 \\
2.575\end{array}$ & $\begin{array}{l}169.1 \\
864.7 \\
2.627\end{array}$ & $\begin{array}{l}172.9 \\
890.0 \\
2.679\end{array}$ & $\begin{array}{l}176.6 \\
915.8 \\
2.731\end{array}$ & $\begin{array}{l}180.4 \\
941.8 \\
2.782\end{array}$ & $\begin{array}{l}184.1 \\
968.3 \\
2.833\end{array}$ & $\begin{array}{l}187.8 \\
995.1 \\
2.884\end{array}$ & $\begin{array}{l}191.5 \\
1022 . \\
2.934\end{array}$ & $\begin{array}{l}195.3 \\
1050 . \\
2.984\end{array}$ \\
\hline 500 & $\begin{array}{l}\text { P } \\
\text { H } \\
\text { S }\end{array}$ & $\begin{array}{l}125.3 \\
789.4 \\
2.435\end{array}$ & $\begin{array}{l}128.4 \\
813.7 \\
2.488\end{array}$ & $\begin{array}{l}131.5 \\
838.4 \\
2.541\end{array}$ & $\begin{array}{l}130.6 \\
863.4 \\
2.593\end{array}$ & $\begin{array}{l}137.6 \\
888.8 \\
2.645\end{array}$ & $\begin{array}{l}140.7 \\
914.6 \\
2.697\end{array}$ & $\begin{array}{l}143.7 \\
940.7 \\
2.748\end{array}$ & $\begin{array}{l}146.7 \\
967.2 \\
2.800\end{array}$ & $\begin{array}{l}149.7 \\
994.1 \\
2.850\end{array}$ & $\begin{array}{l}152.7 \\
1021 . \\
2.901\end{array}$ & $\begin{array}{l}155.7 \\
1049 . \\
2.951\end{array}$ \\
\hline 600 & $\begin{array}{l}\text { P } \\
\text { H } \\
\text { S }\end{array}$ & $\begin{array}{l}103.7 \\
787.9 \\
2.407\end{array}$ & $\begin{array}{l}106.3 \\
812.2 \\
2.460\end{array}$ & $\begin{array}{l}108.9 \\
837.0 \\
2.513\end{array}$ & $\begin{array}{l}111.5 \\
862.1 \\
.2 .565\end{array}$ & $\begin{array}{l}114.1 \\
887.6 \\
2.617\end{array}$ & $\begin{array}{l}116.7 \\
913.4 \\
2.669\end{array}$ & $\begin{array}{l}119.2 \\
939.6 \\
2.721\end{array}$ & $\begin{array}{l}121.7 \\
966.1 \\
2.772\end{array}$ & $\begin{array}{l}124.3 \\
993.0 \\
2.823\end{array}$ & $\begin{array}{l}126.8 \\
1020 . \\
2.874\end{array}$ & $\begin{array}{l}129.3 \\
1048 . \\
2.924\end{array}$ \\
\hline 700 & $\begin{array}{l}y \\
: \\
s\end{array}$ & $\begin{array}{l}88.27 \\
78 \epsilon .3 \\
2.382\end{array}$ & $\begin{array}{l}90.55 \\
810.8 \\
2.436\end{array}$ & $\begin{array}{l}92.81 \\
835.6 \\
2.489\end{array}$ & $\begin{array}{l}95.06 \\
860.8 \\
2.541\end{array}$ & $\begin{array}{l}97.29 \\
886.3 \\
2.594\end{array}$ & $\begin{array}{l}99.51 \\
912.2 \\
2.646\end{array}$ & $\begin{array}{l}101.7 \\
938.4 \\
2.697\end{array}$ & $\begin{array}{l}103.9 \\
965.0 \\
2.748\end{array}$ & $\begin{array}{l}106.1 \\
992.0 \\
2.799\end{array}$ & $\begin{array}{l}108.3 \\
1019 . \\
2.850\end{array}$ & $\begin{array}{l}110.5 \\
1047 \\
2.901\end{array}$ \\
\hline 800 & $\begin{array}{l}\mathbf{Y} \\
\mathrm{E} \\
\mathrm{S}\end{array}$ & $\begin{array}{l}76.67 \\
784.8 \\
2.361\end{array}$ & $\begin{array}{l}78.70 \\
809.3 \\
2.414\end{array}$ & $\begin{array}{l}80.71 \\
834.2 \\
2.467\end{array}$ & $\begin{array}{l}.82 .70 \\
859.5 \\
.2 .520\end{array}$ & $\begin{array}{l}84.68 \\
885.0 \\
2.573\end{array}$ & $\begin{array}{l}86.65 \\
911.0 \\
2.625\end{array}$ & $\begin{array}{l}88.61 \\
937.3 \\
2.676\end{array}$ & $\begin{array}{l}90.56 \\
963.9 \\
2.728\end{array}$ & $\begin{array}{l}92.49 \\
990.9 \\
2.779\end{array}$ & $\begin{array}{l}94.42 \\
1018 . \\
2.830\end{array}$ & $\begin{array}{l}96.34 \\
1046 . \\
2.880\end{array}$ \\
\hline 900 & $\begin{array}{l}8 \\
E \\
\text { S }\end{array}$ & $\begin{array}{l}67.65 \\
783.2 \\
2.341\end{array}$ & $\begin{array}{l}69.48 \\
207.8 \\
2.395\end{array}$ & $\begin{array}{l}71.30 \\
832.8 \\
2.448\end{array}$ & $\begin{array}{l}73.10 \\
858.1 \\
2.501\end{array}$ & $\begin{array}{l}74.88 \\
883.8 \\
2.554\end{array}$ & $\begin{array}{l}76.65 \\
909.8 \\
2.606\end{array}$ & $\begin{array}{l}78.41 \\
936.1 \\
2.658\end{array}$ & $\begin{array}{l}80.16 \\
962.8 \\
2.709\end{array}$ & $\begin{array}{l}81.90 \\
989.9 \\
2.761\end{array}$ & $\begin{array}{l}83.63 \\
1017 \\
2812\end{array}$ & $\begin{array}{l}85.35 \\
1045 . \\
2.862\end{array}$ \\
\hline 1000 & $\begin{array}{l}\mathbf{y} \\
\mathrm{E} \\
\mathrm{S}\end{array}$ & $\begin{array}{l}60.43 \\
781.6 \\
2.324\end{array}$ & $\begin{array}{l}62.11 \\
806.3 \\
2.378\end{array}$ & $\begin{array}{l}63.76 \\
831.4 \\
2.431\end{array}$ & $\begin{array}{l}65.41 \\
856.8 \\
2.484\end{array}$ & $\begin{array}{l}67.03 \\
882.5 \\
2.537\end{array}$ & $\begin{array}{l}68.65 \\
908.6 \\
2.589\end{array}$ & $\begin{array}{l}70.25 \\
935.0 \\
2.641\end{array}$ & $\begin{array}{l}71.84 \\
961.7 \\
2.693\end{array}$ & $\begin{array}{l}73.42 \\
988.8 \\
2.744\end{array}$ & $\begin{array}{l}74.99 \\
1016 . \\
2.795\end{array}$ & $\begin{array}{l}76.56 \\
1044 . \\
2.846\end{array}$ \\
\hline
\end{tabular}


Table 12 (Continued)

Temperature range: 180 to $280^{\circ} \mathrm{C}$

\begin{tabular}{|c|c|c|c|c|c|c|c|c|c|c|c|c|}
\hline $\mathbf{P}$ & $\pi$ & 180 & 190 & 200 & 210 & 220 & 230 & 240 & 250 & 260 & 270 & 280 \\
\hline 1200 & $\begin{array}{l}\text { V } \\
\text { B } \\
\text { s }\end{array}$ & $\begin{array}{l}49.55 \\
778.4 \\
2.292\end{array}$ & $\begin{array}{l}51.03 \\
803.3 \\
2.347\end{array}$ & $\begin{array}{l}52.46 \\
828.5 \\
2.401\end{array}$ & $\begin{array}{l}53.87 \\
854.0 \\
2.454\end{array}$ & $\begin{array}{l}55.26 \\
879.9 \\
2.507\end{array}$ & $\begin{array}{l}56.64 \\
906.1 \\
2.560\end{array}$ & $\begin{array}{l}58.01 \\
932.6 \\
2.612\end{array}$ & $\begin{array}{l}59.36 \\
959.5 \\
2.664\end{array}$ & $\begin{array}{l}60.71 \\
986.7 \\
2.715\end{array}$ & $\begin{array}{l}62.04 \\
1014 . \\
2.766\end{array}$ & $\begin{array}{l}63.37 \\
1042 . \\
2.817\end{array}$ \\
\hline 1400 & $\begin{array}{l}\text { i } \\
\text { i } \\
s\end{array}$ & $\begin{array}{l}41.83 \\
775.0 \\
2.265\end{array}$ & $\begin{array}{l}43.11 \\
800.1 \\
2.320\end{array}$ & $\begin{array}{l}44.38 \\
825.5 \\
2.374\end{array}$ & 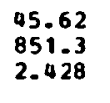 & $\begin{array}{l}46.85 \\
877.3 \\
2.481\end{array}$ & $\begin{array}{l}48.06 \\
903.6 \\
2.534\end{array}$ & $\begin{array}{l}49.26 \\
930.2 \\
2.586\end{array}$ & $\begin{array}{l}50.45 \\
957.2 \\
2.638\end{array}$ & $\begin{array}{l}51.62 \\
984.5 \\
2.690\end{array}$ & $\begin{array}{l}52.79 \\
1012 \\
2.741\end{array}$ & $\begin{array}{l}53.95 \\
1000 . \\
2.792\end{array}$ \\
\hline 1600 & $\begin{array}{l}\text { y } \\
\text { u } \\
\text { s }\end{array}$ & $\begin{array}{l}36.00 \\
771.6 \\
2.240\end{array}$ & $\begin{array}{l}37.16 \\
796.9 \\
2.296\end{array}$ & $\begin{array}{l}38.31 \\
822.5 \\
2.350\end{array}$ & $\begin{array}{l}39.43 \\
848.4 \\
2.404\end{array}$ & $\begin{array}{l}40.53 \\
874.6 \\
2.458\end{array}$ & $\begin{array}{l}41.62 \\
901.1 \\
2.511\end{array}$ & $\begin{array}{l}42.70 \\
927.8 \\
2.564\end{array}$ & $\begin{array}{l}43.76 \\
954.9 \\
2.616\end{array}$ & $\begin{array}{l}44.81 \\
982.3 \\
2.668\end{array}$ & $\begin{array}{l}45.85 \\
10.10 \\
2.719\end{array}$ & $\begin{array}{l}46.89 \\
1038 \\
2.771\end{array}$ \\
\hline 1800 & $\begin{array}{l}\text { H } \\
\text { H }\end{array}$ & $\begin{array}{r}31.45 \\
.768 .1 \\
2.213\end{array}$ & $\begin{array}{l}32.53 \\
793.7 \\
2.273\end{array}$ & $\begin{array}{l}33.58 \\
819.5 \\
2.329\end{array}$ & $\begin{array}{l}34.61 \\
845.5 \\
2.383\end{array}$ & $\begin{array}{l}35.62 \\
871.9 \\
2.437\end{array}$ & $\begin{array}{l}36.61 \\
898.5 \\
2.490\end{array}$ & $\begin{array}{l}37.59 \\
925.4 \\
2.543\end{array}$ & $\begin{array}{l}38.56 \\
952.6 \\
2.596\end{array}$ & $\begin{array}{l}39.51 \\
980.1 \\
2.648\end{array}$ & $\begin{array}{l}40.46 \\
1008 . \\
2.700\end{array}$ & $\begin{array}{l}41.39 \\
1036 . \\
2.751\end{array}$ \\
\hline 2000 & $\begin{array}{l}\vec{r} \\
H \\
S\end{array}$ & $\begin{array}{l}27.81 \\
764-5 \\
2.197\end{array}$ & $\begin{array}{l}28.81 \\
790.3 \\
2.253\end{array}$ & $\begin{array}{l}29.79 \\
816.3 \\
2.309\end{array}$ & $\begin{array}{l}30.75 \\
842.6 \\
2.363\end{array}$ & $\begin{array}{l}31.68 \\
869.1 \\
2.418\end{array}$ & $\begin{array}{l}32.60 \\
895.9 \\
2.472\end{array}$ & $\begin{array}{l}33.50 \\
922.9 \\
2.525\end{array}$ & $\begin{array}{l}34.39 \\
950.2 \\
2.578\end{array}$ & $\begin{array}{l}35.27 \\
977.9 \\
2.630\end{array}$ & $\begin{array}{l}36-14 \\
1006 . \\
2.682\end{array}$ & $\begin{array}{l}37 . c 0 \\
1034 . \\
2.733\end{array}$ \\
\hline$\Sigma 100$ & $\begin{array}{l}\mathrm{T} \\
\mathrm{H} \\
\mathrm{s}\end{array}$ & $\begin{array}{l}26.24 \\
76.2 .6 \\
2.187\end{array}$ & $\begin{array}{l}27.22 \\
788.6 \\
2.243\end{array}$ & $\begin{array}{l}28.17 \\
814.7 \\
2.299\end{array}$ & $\begin{array}{l}29.09 \\
84 i .1 \\
2.354\end{array}$ & $\begin{array}{l}30.00 \\
867.7 \\
2.409\end{array}$ & $\begin{array}{l}30.88 \\
894.5 \\
2.463\end{array}$ & $\begin{array}{l}31.75 \\
921.6 \\
2.516\end{array}$ & $\begin{array}{l}32.61 \\
949.0 \\
2.569\end{array}$ & $\begin{array}{l}33.46 \\
976.7 \\
2.621\end{array}$ & $\begin{array}{l}34.29 \\
1005 \\
2.673\end{array}$ & $\begin{array}{l}35.12 \\
1033 . \\
2.725\end{array}$ \\
\hline 2200 & $\begin{array}{l}\mathrm{v} \\
\mathrm{y} \\
\mathrm{s}\end{array}$ & $\begin{array}{l}24.82 \\
76 C_{-8} \\
2.177\end{array}$ & $\begin{array}{l}25.77 \\
786.8 \\
2.234\end{array}$ & $\begin{array}{l}26.69 \\
813.1 \\
2.290\end{array}$ & $\begin{array}{l}27.58 \\
839.6 \\
2.345\end{array}$ & $\begin{array}{l}28.406 \\
866.3 \\
2.400\end{array}$ & $\begin{array}{l}29.32 \\
893.2 \\
2.454\end{array}$ & $\begin{array}{l}30.16 \\
920.4 \\
2.507\end{array}$ & $\begin{array}{r}30.99 \\
947.8 \\
.2 .560\end{array}$ & $\begin{array}{l}31.80 \\
975.6 \\
2.613\end{array}$ & $\begin{array}{l}32.61 \\
1004 \\
2.665\end{array}$ & $\begin{array}{l}33.40 \\
1032 \\
2.717\end{array}$ \\
\hline 2300 & $\begin{array}{l}\mathrm{V} \\
\mathrm{H} \\
\mathrm{S}\end{array}$ & $\begin{array}{l}23.51 \\
75 e^{-.8} \\
2.167\end{array}$ & $\begin{array}{l}24.44 \\
785.1 \\
2.225\end{array}$ & $\begin{array}{l}25.34 \\
811.5 \\
2.281\end{array}$ & $\begin{array}{l}26.21 \\
838.0 \\
2.336\end{array}$ & $\begin{array}{l}27.06 \\
864.8 \\
2.391\end{array}$ & $\begin{array}{l}27.89 \\
891.8 \\
2.446\end{array}$ & $\begin{array}{l}28.71 \\
919.1 \\
2.499\end{array}$ & $\begin{array}{l}29.51 \\
946.6 \\
2.552\end{array}$ & $\begin{array}{l}30.30 \\
974.4 \\
2.605\end{array}$ & $\begin{array}{l}31.07 \\
1003 \\
2.657\end{array}$ & $\begin{array}{l}31.84 \\
1031 . \\
2.709\end{array}$ \\
\hline 2400 & $\begin{array}{l}\mathrm{V} \\
\mathrm{H} \\
\mathrm{S}\end{array}$ & $\begin{array}{l}22.31 \\
756.9 \\
2.158\end{array}$ & $\begin{array}{l}23.22 \\
783.3 \\
2.216\end{array}$ & $\begin{array}{l}24.10 \\
809.8 \\
2.272\end{array}$ & $\begin{array}{l}24.95 \\
836.5 \\
2.328\end{array}$ & $\begin{array}{l}25.77 \\
863.4 \\
2.383\end{array}$ & $\begin{array}{l}26.58 \\
890.5 \\
2.438\end{array}$ & $\begin{array}{l}27.37 \\
997.8 \\
2.491\end{array}$ & $\begin{array}{l}28.15 \\
945.4 \\
2.545\end{array}$ & $\begin{array}{l}28.91 \\
973.3 \\
2.597\end{array}$ & $\begin{array}{l}29.67 \\
1001 . \\
2.650\end{array}$ & $\begin{array}{l}30.41 \\
.1030 \\
2.701\end{array}$ \\
\hline 2500 & $\begin{array}{l}\text { H } \\
\text { H }\end{array}$ & $\begin{array}{l}21.21 \\
754.9 \\
2.149\end{array}$ & $\begin{array}{l}22.10 \\
781.5 \\
2.207\end{array}$ & $\begin{array}{l}22.95 \\
808.1 \\
2.264\end{array}$ & $\begin{array}{r}23.78 \\
836.9 \\
.2 .320\end{array}$ & $\begin{array}{l}24.59 \\
861.9 \\
2.375\end{array}$ & $\begin{array}{l}25.38 \\
889.1 \\
2.430\end{array}$ & $\begin{array}{l}26.15 \\
916.5 \\
.2 .484\end{array}$ & $\begin{array}{l}26.90 \\
944.2 \\
.2 .537\end{array}$ & $\begin{array}{l}27.64 \\
972.1 \\
2.590\end{array}$ & $\begin{array}{l}28.37 \\
1000 . \\
2.692\end{array}$ & $\begin{array}{l}29.09 \\
1029 . \\
2.699\end{array}$ \\
\hline 2600 & $\begin{array}{l}\mathrm{y} \\
\mathrm{u} \\
\mathrm{s}\end{array}$ & $\begin{array}{l}20.19 \\
752.9 \\
2.140\end{array}$ & $\begin{array}{l}21.06 \\
779.6 \\
2.198\end{array}$ & $\begin{array}{l}21.90 \\
806.4 \\
2.255\end{array}$ & $\begin{array}{l}22.71 \\
833.4 \\
2.312\end{array}$ & $\begin{array}{l}23.50 \\
860.4 \\
2.367\end{array}$ & $\begin{array}{l}24.26 \\
887.7 \\
2.422\end{array}$ & $\begin{array}{l}25.01 \\
915.2 \\
2.476\end{array}$ & $\begin{array}{l}25.75 \\
943.0 \\
2.530\end{array}$ & $\begin{array}{l}26.47 \\
971.0 \\
2.583\end{array}$ & $\begin{array}{l}27.18 \\
99.9 .2 \\
2.635\end{array}$ & $\begin{array}{l}27.87 \\
1028 . \\
2.687\end{array}$ \\
\hline 2700 & $\begin{array}{l}\text { H } \\
\mathrm{H}\end{array}$ & $\begin{array}{l}19.24 \\
750.9 \\
2.131\end{array}$ & $\begin{array}{l}20.10 \\
777.8 \\
2.190\end{array}$ & $\begin{array}{l}20.92 \\
804.7 \\
2.247\end{array}$ & $\begin{array}{l}21.71 \\
831.8 \\
2.304\end{array}$ & $\begin{array}{l}22.48 \\
859.0 \\
2.359\end{array}$ & $\begin{array}{l}23.23 \\
886.3 \\
2.414\end{array}$ & $\begin{array}{l}23.96 \\
913.9 \\
2.469\end{array}$ & $\begin{array}{l}24.68 \\
941.8 \\
2.522\end{array}$ & $\begin{array}{l}25.38 \\
969.8 \\
2.576\end{array}$ & $\begin{array}{l}26.07 \\
998.1 \\
2.628\end{array}$ & $\begin{array}{l}26.75 \\
1027 . \\
2.680\end{array}$ \\
\hline 2800 & $\begin{array}{l}\text { y } \\
\text { H } \\
\text { S }\end{array}$ & $\begin{array}{l}18.35 \\
748.8 \\
2.122\end{array}$ & $\begin{array}{l}79.20 \\
775.9 \\
2.181\end{array}$ & $\begin{array}{l}20.01 \\
803.0 \\
2.239\end{array}$ & $\begin{array}{l}20.79 \\
830.1 \\
2.296\end{array}$ & $\begin{array}{l}21.54 \\
857.5 \\
2.352\end{array}$ & $\begin{array}{l}22.28 \\
884.9 \\
2.407\end{array}$ & $\begin{array}{l}22.99 \\
912.6 \\
2.462\end{array}$ & $\begin{array}{l}23.69 \\
940.5 \\
2.515\end{array}$ & $\begin{array}{l}24.37 \\
968.6 \\
2.569\end{array}$ & $\begin{array}{l}25.04 \\
997.0 \\
2.621\end{array}$ & $\begin{array}{l}25.70 \\
1026 . \\
2.679\end{array}$ \\
\hline
\end{tabular}


Table i 2 (Continued)

Temperature range: 180 to $280^{\circ} \mathrm{C}$

\begin{tabular}{|c|c|c|c|c|c|c|c|c|c|c|c|c|c|}
\hline $\mathbf{P}$ & & $\mathbf{T}$ & 180 & 190 & 200 & 210 & 220 & 230 & 240 & 250 & 260 & 270 & 280 \\
\hline 2900 & $\begin{array}{l}\mathbf{Y} \\
\mathbf{H} \\
\mathbf{S}\end{array}$ & & $\begin{array}{l}17.53 \\
74.6 .7 \\
2.114\end{array}$ & $\begin{array}{l}18.36 \\
773.9 \\
2.173\end{array}$ & $\begin{array}{l}19.16 \\
801.2 \\
2.231\end{array}$ & $\begin{array}{l}19.93 \\
828.5 \\
2.288\end{array}$ & $\begin{array}{l}20.67 \\
855.9 \\
2.345\end{array}$ & $\begin{array}{l}21.38 \\
883.5 \\
2.400\end{array}$ & $\begin{array}{l}22.08 \\
911.3 \\
2.455\end{array}$ & $\begin{array}{l}22.76 \\
939.3 \\
2.509\end{array}$ & $\begin{array}{l}23.43 \\
967.5 \\
2.562\end{array}$ & $\begin{array}{l}24.09 \\
995.9 \\
2.615\end{array}$ & $\begin{array}{l}24.73 \\
1025 . \\
2.667\end{array}$ \\
\hline 3000 & $\begin{array}{l}\mathbf{V} \\
\mathbf{H} \\
\mathbf{S}\end{array}$ & & $\begin{array}{l}16.75 \\
744.5 \\
2.105\end{array}$ & $\begin{array}{l}17.58 \\
77 \overline{5} .0 \\
2.165\end{array}$ & $\begin{array}{l}18.37 \\
799.4 \\
2.224\end{array}$ & $\begin{array}{l}19.12 \\
826.9 \\
2.281\end{array}$ & $\begin{array}{l}19.85 \\
854.4 \\
2.337\end{array}$ & $\begin{array}{l}20.55 \\
882.1 \\
2.393\end{array}$ & $\begin{array}{l}21.23 \\
910.0 \\
2.448\end{array}$ & $\begin{array}{l}21.90 \\
938.0 \\
2.502\end{array}$ & $\begin{array}{l}22.55 \\
96 \epsilon .3 \\
2.556\end{array}$ & $\begin{array}{l}23.19 \\
994.8 \\
2.608\end{array}$ & $\begin{array}{l}23.82 \\
1024 . \\
2.661\end{array}$ \\
\hline 3100 & $\begin{array}{l}7 \\
4 \\
5\end{array}$ & & $\begin{array}{l}16.03 \\
742.3 \\
2.096\end{array}$ & $\begin{array}{l}16.85 \\
770.0 \\
2.157\end{array}$ & $\begin{array}{l}17.63 \\
797.6 \\
2.216\end{array}$ & $\begin{array}{l}18.37 \\
825.2 \\
2.274\end{array}$ & $\begin{array}{l}19.08 \\
852.9 \\
2.330\end{array}$ & $\begin{array}{l}19.77 \\
880.7 \\
2.386\end{array}$ & $\begin{array}{l}20.44 \\
908.6 \\
2.441\end{array}$ & $\begin{array}{l}21.10 \\
936.8 \\
2.495\end{array}$ & $\begin{array}{l}21.73 \\
965.1 \\
2.549\end{array}$ & $\begin{array}{l}22.36 \\
993.7 \\
2.602\end{array}$ & $\begin{array}{l}22.97 \\
1022 . \\
2.655\end{array}$ \\
\hline 3200 & $\begin{array}{l}5 \\
4 \\
5\end{array}$ & & $\begin{array}{l}15.35 \\
740.0 \\
2.088\end{array}$ & $\begin{array}{l}16.16 \\
768.0 \\
2.549\end{array}$ & $\begin{array}{l}16.93 \\
79.5 .8 \\
2.208\end{array}$ & $\begin{array}{l}17.66 \\
823.5 \\
2.266\end{array}$ & $\begin{array}{l}18.36 \\
8511.3 \\
2.323\end{array}$ & $\begin{array}{l}19.04 \\
879.2 \\
2.379\end{array}$ & $\begin{array}{l}19.70 \\
907.3 \\
2.435\end{array}$ & $\begin{array}{l}20.34 \\
935.5 \\
2.489\end{array}$ & $\begin{array}{l}20.97 \\
963.9 \\
2.543\end{array}$ & $\begin{array}{l}21.58 \\
992.5 \\
2.596\end{array}$ & $\begin{array}{l}22.18 \\
1021 . \\
2.649\end{array}$ \\
\hline 3300 & $\begin{array}{l}y \\
\mathbf{1} \\
\mathbf{3}\end{array}$ & & $\begin{array}{l}14.70 \\
737.7 \\
2.080\end{array}$ & $\begin{array}{l}15.51 \\
765.9 \\
2.141 .\end{array}$ & $\begin{array}{l}16.27 \\
793.9 \\
2.201\end{array}$ & $\begin{array}{l}17.00 \\
821.8 \\
2.259\end{array}$ & $\begin{array}{l}17.69 \\
849.7 \\
2.317\end{array}$ & $\begin{array}{l}18.36 \\
877.8 \\
2.373\end{array}$ & $\begin{array}{l}19.00 \\
905.9 \\
2.428\end{array}$ & $\begin{array}{l}19.63 \\
934.2 \\
2.483\end{array}$ & $\begin{array}{l}20.24 \\
962.7 \\
2.537\end{array}$ & $\begin{array}{l}20.84 \\
991.4 \\
2.590\end{array}$ & $\begin{array}{l}21.43 \\
1020 . \\
2.643\end{array}$ \\
\hline 3400 & $\begin{array}{l}7 \\
H \\
S\end{array}$ & & $\begin{array}{l}14.10 \\
735.4 \\
2.071\end{array}$ & $\begin{array}{l}14.90 \\
763: 8 \\
2.133\end{array}$ & $\begin{array}{l}15.65 \\
792.0 \\
2.194\end{array}$ & $\begin{array}{l}16.37 \\
820.1 \\
2.252\end{array}$ & $\begin{array}{l}17.05 \\
848.2 \\
2.310\end{array}$ & $\begin{array}{l}17.71 \\
876.3 \\
2.366\end{array}$ & $\begin{array}{l}18.34 \\
904-5 \\
2.422\end{array}$ & $\begin{array}{l}18.96 \\
932.9 \\
2.477\end{array}$ & $\begin{array}{l}19.56 \\
961.5 \\
2.531\end{array}$ & $\begin{array}{l}20.15 \\
990.2 \\
2.584\end{array}$ & $\begin{array}{l}20.73 \\
1019 \\
2.637\end{array}$ \\
\hline 3500 & $\begin{array}{l}7 \\
\mathbf{u} \\
\mathbf{S}\end{array}$ & & $\begin{array}{l}13.52 \\
333.0 \\
2.063\end{array}$ & $\begin{array}{l}14.32 \\
761.7 \\
2.126\end{array}$ & $\begin{array}{l}15.07 \\
790.1 \\
2.186\end{array}$ & $\begin{array}{l}15.78 \\
81.3 .3 \\
2.245\end{array}$ & $\begin{array}{l}16.45 \\
846.6 \\
2.303\end{array}$ & $\begin{array}{l}17.10 \\
874.8 \\
2.360\end{array}$ & $\begin{array}{l}17.73 \\
903.1 \\
2.416\end{array}$ & $\begin{array}{l}18.33 \\
931.6 \\
2.471\end{array}$ & $\begin{array}{l}18.92 \\
960.3 \\
2.525\end{array}$ & $\begin{array}{l}19.50 \\
989.1 \\
2.578\end{array}$ & $\begin{array}{l}20.06 \\
10.18 \\
2: 631\end{array}$ \\
\hline 3600 & $\begin{array}{l}1 \\
8 \\
5\end{array}$ & & $\begin{array}{l}12.98 \\
730.5 \\
2.055\end{array}$ & $\begin{array}{l}13.78 \\
759.5 \\
2.118\end{array}$ & $\begin{array}{l}14.52 \\
788.2 \\
2.179\end{array}$ & $\begin{array}{l}15.22 \\
816.6 \\
2.239\end{array}$ & $\begin{array}{l}15.89 \\
844.9 \\
2.297\end{array}$ & $\begin{array}{l}16.52 \\
873.3 \\
2.354\end{array}$ & $\begin{array}{l}17.14 \\
901.8 \\
2.410\end{array}$ & $\begin{array}{l}17.74 \\
930.3 \\
2.465\end{array}$ & $\begin{array}{l}18.32 \\
959.0 \\
2.519\end{array}$ & $\begin{array}{l}18.88 \\
987.9 \\
2573\end{array}$ & $\begin{array}{l}19.44 \\
1.017 . \\
2.626\end{array}$ \\
\hline 3700 & $\begin{array}{l}\mid \overrightarrow{1} \\
\mathbf{s}\end{array}$ & & $\begin{array}{l}12.46 \\
728.0 \\
2.046\end{array}$ & $\begin{array}{l}13.26 \\
757.3 \\
2.110\end{array}$ & $\begin{array}{l}14.00 \\
786.2 \\
2.172\end{array}$ & $\begin{array}{l}14.69 \\
81.48 \\
2.232\end{array}$ & $\begin{array}{l}15.35 \\
843.3 \\
2.290\end{array}$ & $\begin{array}{l}15.98 \\
871.8 \\
2.347\end{array}$ & $\begin{array}{l}16.59 \\
900.4 \\
2.404\end{array}$ & $\begin{array}{l}17.18 \\
929.0 \\
2.459\end{array}$ & $\begin{array}{l}17.75 \\
957.8 \\
2.513\end{array}$ & $\begin{array}{l}18.30 \\
986.8 \\
2.567\end{array}$ & $\begin{array}{l}18.85 \\
1016 . \\
2.620\end{array}$ \\
\hline 3800 & $\begin{array}{l}\text { i } \\
\text { s }\end{array}$ & & $\begin{array}{l}11.96 \\
725.5 \\
2.038\end{array}$ & $\begin{array}{r}12.76 \\
755.1 \\
2.1 .03\end{array}$ & $\begin{array}{l}13.50 \\
78.42 \\
2.165\end{array}$ & $\begin{array}{l}14.19 \\
813.0 \\
2.225\end{array}$ & $\begin{array}{l}14.84 \\
841.7 \\
2.284\end{array}$ & $\begin{array}{l}15.46 \\
870.3 \\
2.341\end{array}$ & $\begin{array}{l}16.06 \\
898.9 \\
2.398\end{array}$ & $\begin{array}{l}16.64 \\
927.7 \\
2.453\end{array}$ & $\begin{array}{l}17.20 \\
956.6 \\
2.508\end{array}$ & $\begin{array}{l}17.75 \\
985.6 \\
2.562\end{array}$ & $\begin{array}{l}18.29 \\
1015 . \\
2.615\end{array}$ \\
\hline 3900 & $\begin{array}{l}P \\
H \\
\text { S }\end{array}$ & & $\begin{array}{l}11.49 \\
722.8 \\
2.030\end{array}$ & $\begin{array}{l}12.29 \\
752.8 \\
2.075\end{array}$ & $\begin{array}{l}13.03 \\
782.2 \\
2.158\end{array}$ & $\begin{array}{l}13.71 \\
811.2 \\
2.218\end{array}$ & $\begin{array}{l}14.36 \\
840.0 \\
2.277\end{array}$ & $\begin{array}{l}14.97 \\
868.7 \\
2.335\end{array}$ & $\begin{array}{l}15.57 \\
897.5 \\
2.392\end{array}$ & $\begin{array}{l}16.14 \\
926.4 \\
2.447\end{array}$ & $\begin{array}{l}16.69 \\
955.3 \\
2.502\end{array}$ & $\begin{array}{l}17.23 \\
989.5 \\
2.556\end{array}$ & $\begin{array}{l}17.76 \\
1014 . \\
2.610\end{array}$ \\
\hline 4000 & $\begin{array}{l}\mathbf{S} \\
\mathbf{t} \\
\mathbf{S}\end{array}$ & & $\begin{array}{l}11.05 \\
720-1 \\
2.021\end{array}$ & $\begin{array}{l}11.85 \\
750.5 \\
2.087\end{array}$ & $\begin{array}{l}12.58 \\
780.1 \\
2.151\end{array}$ & $\begin{array}{l}13.26 \\
809.3 \\
2.212\end{array}$ & $\begin{array}{l}13.90 \\
838.3 \\
2.271\end{array}$ & $\begin{array}{l}14.51 \\
867.2 \\
2.329\end{array}$ & $\begin{array}{l}15.09 \\
896.1 \\
2.386\end{array}$ & $\begin{array}{l}15.66 \\
925.0 \\
2.442\end{array}$ & $\begin{array}{l}16.20 \\
954.1 \\
2.497\end{array}$ & $\begin{array}{l}16.73 \\
983.3 \\
2.551\end{array}$ & $\begin{array}{l}17.25 \\
1013 . \\
2.605\end{array}$ \\
\hline 4100 & $\begin{array}{l}\mathbf{D} \\
\mathrm{H} \\
\mathrm{S}\end{array}$ & & $\begin{array}{l}10.62 \\
717.4 \\
2.013\end{array}$ & $\begin{array}{l}11.42 \\
748.1 \\
2.080\end{array}$ & $\begin{array}{l}12.15 \\
778.0 \\
2.144\end{array}$ & $\begin{array}{l}12.83 \\
807.5 \\
2.205\end{array}$ & $\begin{array}{l}13.46 \\
836.6 \\
2.265\end{array}$ & $\begin{array}{l}14.07 \\
865.6 \\
2.323\end{array}$ & $\begin{array}{l}14.64 \\
894.7 \\
2.380\end{array}$ & $\begin{array}{l}15.20 \\
923.7 \\
2.436\end{array}$ & $\begin{array}{l}15.74 \\
952.9 \\
2.492\end{array}$ & $\begin{array}{l}16.26 \\
982.1 \\
2.546\end{array}$ & $\begin{array}{l}16.7 n \\
1012 . \\
2.600\end{array}$ \\
\hline
\end{tabular}


Table 12 (Continued)

Temperature range: 180 to $280^{\circ} \mathrm{C}$

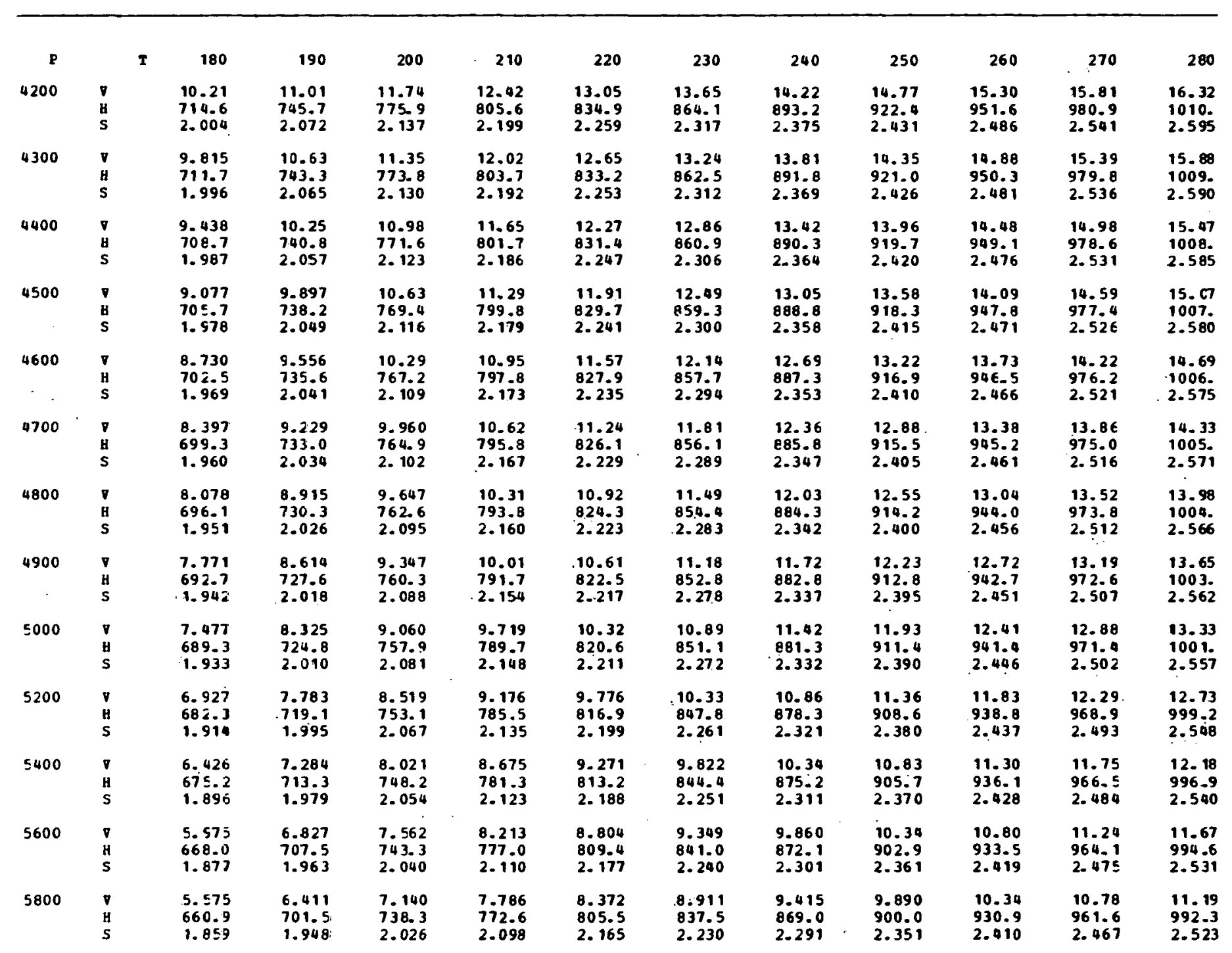


Table 12 (Continued)

Terperature range: 180 to $280^{\circ} \mathrm{C}$

\begin{tabular}{|c|c|c|c|c|c|c|c|c|c|c|c|c|c|}
\hline P & & $\mathbf{T}$ & 180 & 190 & 200 & 210 & 220 & 230 & 240 & 250 & 260 & 270 & 280 \\
\hline 6000 & $\begin{array}{l}\mathbf{Y} \\
\mathbf{B} \\
\mathbf{S}\end{array}$ & & $\begin{array}{l}5.224 \\
654.0 \\
1.841\end{array}$ & $\begin{array}{l}6.034 \\
695.6 \\
1.932\end{array}$ & $\begin{array}{l}6.752 \\
733.2 \\
2.012\end{array}$ & $\begin{array}{l}7.392 \\
768.2 \\
2.086\end{array}$ & $\begin{array}{l}7.972 \\
8091.7 \\
2.154\end{array}$ & $\begin{array}{l}8.505 \\
834.1 \\
2.219\end{array}$ & $\begin{array}{l}9.002 \\
865.8 \\
2.282\end{array}$ & $\begin{array}{l}9.470 \\
897.2 \\
2.342\end{array}$ & $\begin{array}{l}9.915 \\
928.2 \\
2.401\end{array}$ & $\begin{array}{l}10.34 \\
959.2 \\
2.458\end{array}$ & $\begin{array}{l}10.75 \\
990.0 \\
2.515\end{array}$ \\
\hline 6200 & $\begin{array}{l}\mathbf{V} \\
\mathbf{E} \\
\mathbf{S}\end{array}$ & & $\begin{array}{l}4.919 \\
647.5 \\
1.825\end{array}$ & $\begin{array}{l}5.695 \\
689.8 \\
1.917\end{array}$ & $\begin{array}{l}6.398 \\
728.2 \\
1.999\end{array}$ & $\begin{array}{l}7.029 \\
763.9 \\
2.074\end{array}$ & $\begin{array}{l}7.601 \\
797.8 \\
2.143\end{array}$ & $\begin{array}{l}8.128 \\
830.6 \\
2.209\end{array}$ & $\begin{array}{l}8.618 \\
862.7 \\
2.272\end{array}$ & $\begin{array}{l}9.080 \\
894.3 \\
2.333\end{array}$ & $\begin{array}{l}9.518 \\
925.6 \\
2.392\end{array}$ & $\begin{array}{l}9.936 \\
956.7 \\
2.450\end{array}$ & $\begin{array}{l}10.34 \\
987.7 \\
2.507\end{array}$ \\
\hline$\epsilon \infty 00$ & $\begin{array}{l}\mathbf{Y} \\
\mathbf{E} \\
\mathbf{S}\end{array}$ & & $\begin{array}{l}4.656 \\
641.5 \\
1.809\end{array}$ & $\begin{array}{l}5.392 \\
684.2 \\
1.902\end{array}$ & $\begin{array}{l}6.074 \\
723.2 \\
1.986\end{array}$ & $\begin{array}{l}6.693 \\
759.5 \\
2.062\end{array}$ & $\begin{array}{l}7.258 \\
793.9 \\
2.132\end{array}$ & $\begin{array}{l}7.777 \\
827.1 \\
2.199\end{array}$ & $\begin{array}{l}8.261 \\
859.5 \\
2.263\end{array}$ & $\begin{array}{l}8.716 \\
891.4 \\
2.324\end{array}$ & $\begin{array}{l}9.147 \\
922.9 \\
2.384\end{array}$ & $\begin{array}{l}9.559 \\
954.2 \\
2.442\end{array}$ & $\begin{array}{l}9.954 \\
985.4 \\
2.499\end{array}$ \\
\hline 6600 & $\begin{array}{l}\mathbf{Y} \\
\mathbf{H} \\
\mathbf{S}\end{array}$ & & $\begin{array}{l}4.429 \\
635.8 \\
1.795\end{array}$ & $\begin{array}{l}5.122 \\
678.8 \\
1.889\end{array}$ & $\begin{array}{l}5.780 \\
718.3 \\
1.973\end{array}$ & $\begin{array}{l}6.385 \\
755.1 \\
2.050\end{array}$ & $\begin{array}{l}6.940 \\
790.1 \\
2.122\end{array}$ & $\begin{array}{l}7.452 \\
823.7 \\
2.189\end{array}$ & $\begin{array}{l}7.928 \\
856.4 \\
2.253\end{array}$ & $\begin{array}{l}8.377 \\
888.5 \\
2.315\end{array}$ & $\begin{array}{l}8.801 \\
920.3 \\
2.376\end{array}$ & $\begin{array}{l}9.206 \\
951.8 \\
2.434\end{array}$ & $\begin{array}{l}9.594 \\
983.1 \\
2.491\end{array}$ \\
\hline 6800 & $\begin{array}{l}y \\
H \\
S\end{array}$ & & $\begin{array}{l}4.234 \\
630.6 \\
1.781\end{array}$ & $\begin{array}{l}4.882 \\
673.6 \\
1.875\end{array}$ & $\begin{array}{l}5.513 \\
713.6 \\
1.961\end{array}$ & $\begin{array}{l}6.102 \\
750.9 \\
2.039\end{array}$ & $\begin{array}{l}6.646 \\
786.2 \\
2.111\end{array}$ & $\begin{array}{l}7.149 \\
820.2 \\
2.179\end{array}$ & $\begin{array}{l}7.618 \\
853.2 \\
2.244\end{array}$ & $\begin{array}{l}8.060 \\
885.6 \\
2.307\end{array}$ & $\begin{array}{l}8.477 \\
9: 7.6 \\
2.367\end{array}$ & $\begin{array}{l}8.876 \\
949.3 \\
2.426\end{array}$ & $\begin{array}{l}9.257 \\
980.8 \\
2.484\end{array}$ \\
\hline 7000 & $\begin{array}{l}\mathbf{D} \\
\mathbf{B} \\
\mathbf{S}\end{array}$ & & $\begin{array}{l}4.065 \\
625.9 \\
1.769\end{array}$ & $\begin{array}{l}4.670 \\
668.8 \\
1.863\end{array}$ & $\begin{array}{l}5.271 \\
709.0 \\
1.949\end{array}$ & $\begin{array}{l}5.842 \\
746.7 \\
2.027\end{array}$ & $\begin{array}{l}6.373 \\
782.4 \\
2.101\end{array}$ & $\begin{array}{l}6.868 \\
816.8 \\
2.170\end{array}$ & $\begin{array}{l}7.329 \\
850.1 \\
2.235\end{array}$ & $\begin{array}{l}7.763 \\
882.8 \\
2.298\end{array}$ & $\begin{array}{l}8.174 \\
915.0 \\
2.359\end{array}$ & $\begin{array}{l}8.56 \epsilon \\
946.9 \\
2.419\end{array}$ & $\begin{array}{l}8.941 \\
978.6 \\
2.476\end{array}$ \\
\hline 7500 & $\begin{array}{l}\mathbf{P} \\
\mathrm{H} \\
\mathrm{S}\end{array}$ & & $\begin{array}{l}3.733 \\
615.7 \\
1.742\end{array}$ & $\begin{array}{l}4.240 \\
657.9 \\
1.834\end{array}$ & $\begin{array}{l}4.766 \\
698.3 \\
1.921\end{array}$ & $\begin{array}{l}5.285 \\
736.6 \\
2.001\end{array}$ & $\begin{array}{l}5.780 \\
773.2 \\
2.076\end{array}$ & $\begin{array}{l}6.248 \\
808.3 \\
2.146\end{array}$ & $\begin{array}{l}6.688 \\
842.4 \\
2.213\end{array}$ & $\begin{array}{l}7.103 \\
875.7 \\
2.278\end{array}$ & $\begin{array}{l}7.498 \\
9 C 8.4 \\
2.340\end{array}$ & $\begin{array}{l}7.873 \\
940.8 \\
2.400\end{array}$ & $\begin{array}{l}8.233 \\
972.9 \\
2.458\end{array}$ \\
\hline 8000 & $\begin{array}{l}P \\
H \\
S\end{array}$ & & $\begin{array}{l}3.495 \\
607.6 \\
1.720\end{array}$ & $\begin{array}{l}3.919 \\
648.8 \\
1.810\end{array}$ & $\begin{array}{l}4.376 \\
688.9 \\
1.896\end{array}$ & $\begin{array}{l}4.840 \\
727.4 \\
1.977\end{array}$ & $\begin{array}{l}5.294 \\
764.5 \\
2.053\end{array}$ & $\begin{array}{l}5.731 \\
800.2 \\
2.124\end{array}$ & $\begin{array}{l}6.148 \\
834.9 \\
2.193\end{array}$ & $\begin{array}{l}6.543 \\
868.7 \\
2.258\end{array}$ & $\begin{array}{l}6.920 \\
962.0 \\
2.321\end{array}$ & $\begin{array}{l}7.280 \\
934.8 \\
2.382\end{array}$ & $\begin{array}{l}7.625 \\
967.3 \\
2.441\end{array}$ \\
\hline 8500 & $\begin{array}{l}\nabla \\
y \\
5\end{array}$ & & $\begin{array}{l}3.317 \\
601.1 \\
1.702\end{array}$ & $\begin{array}{l}3.676 \\
641.1 \\
1.790\end{array}$ & $\begin{array}{l}4.072 \\
680.7 \\
1.874\end{array}$ & $\begin{array}{l}4.484 \\
719.2 \\
1.955\end{array}$ & $\begin{array}{l}4.897 \\
756.5 \\
2.031\end{array}$ & $\begin{array}{l}5.302 \\
792.6 \\
2.104\end{array}$ & $\begin{array}{l}5.692 \\
827.7 \\
2.173\end{array}$ & $\begin{array}{l}6.066 \\
862.1 \\
2.239\end{array}$ & $\begin{array}{l}6.425 \\
855.7 \\
2.303\end{array}$ & $\begin{array}{l}6.768 \\
929.0 \\
2.365\end{array}$ & $\begin{array}{l}7.098 \\
961.8 \\
2.425\end{array}$ \\
\hline 9030 & $\begin{array}{l}7 \\
H \\
5\end{array}$ & & $\begin{array}{l}3.181 \\
595.8 \\
1.687\end{array}$ & $\begin{array}{l}3.48 .7 \\
6.34 .7 \\
1.772\end{array}$ & $\begin{array}{l}3.832 \\
673.6 \\
1.855\end{array}$ & $\begin{array}{l}4.198 \\
711.9 \\
1.935\end{array}$ & $\begin{array}{l}4.572 \\
749.2 \\
2.012\end{array}$ & $\begin{array}{l}4.943 \\
785.5 \\
2.085\end{array}$ & $\begin{array}{l}5.307 \\
821.0 \\
2.154\end{array}$ & $\begin{array}{l}5.659 \\
855.7 \\
2.221\end{array}$ & $\begin{array}{l}5.999 \\
8 E 9.7 \\
2.286\end{array}$ & $\begin{array}{l}6.326 \\
923 . \Xi \\
2.348\end{array}$ & $\begin{array}{l}6.641 \\
956.5 \\
2.409\end{array}$ \\
\hline 100.30 & $\begin{array}{l}\mathbf{V} \\
\mathbf{H} \\
\mathbf{S}\end{array}$ & & $\begin{array}{l}2.985 \\
587.8 \\
1.663\end{array}$ & $\begin{array}{l}3.217 \\
624.8 \\
1.743\end{array}$ & $\begin{array}{l}3.483 \\
662.2 \\
1.823\end{array}$ & $\begin{array}{l}3.774 \\
699.7 \\
1.902\end{array}$ & $\begin{array}{l}4.080 \\
736.7 \\
1.978\end{array}$ & $\begin{array}{l}4.392 \\
773.1 \\
2.051\end{array}$ & $\begin{array}{l}4.704 \\
808.8 \\
2.121\end{array}$ & $\begin{array}{l}5.012 \\
843.9 \\
2.189\end{array}$ & $\begin{array}{l}5.314 \\
878.5 \\
2.254\end{array}$ & $\begin{array}{l}5.609 \\
912.7 \\
2.318\end{array}$ & $\begin{array}{l}5.895 \\
946.4 \\
2.379\end{array}$ \\
\hline 110000 & $\begin{array}{l}Y \\
H \\
S\end{array}$ & & $\begin{array}{l}2.850 \\
582.1 \\
1.644\end{array}$ & $\begin{array}{l}3.0 \equiv 4 \\
617.6 \\
1.7=1\end{array}$ & $\begin{array}{l}3.246 \\
653.7 \\
1.798\end{array}$ & $\begin{array}{l}3.482 \\
690.2 \\
1.875\end{array}$ & $\begin{array}{l}3.734 \\
726.6 \\
.1 .949\end{array}$ & $\begin{array}{l}3.996 \\
762.8 \\
2.022\end{array}$ & $\begin{array}{l}4.264 \\
798.5 \\
2.092\end{array}$ & $\begin{array}{l}4.532 \\
833.7 \\
2.160\end{array}$ & $\begin{array}{l}4.798 \\
86.8 .6 \\
2.226\end{array}$ & $\begin{array}{l}5.061 \\
903.0 \\
2.290\end{array}$ & $\begin{array}{l}5.319 \\
937.2 \\
2.352\end{array}$ \\
\hline 12000 & $\begin{array}{l}V \\
H \\
S\end{array}$ & & $\begin{array}{l}2.749 \\
577.8 \\
1.628\end{array}$ & $\begin{array}{l}2.962 \\
612.2 \\
1.763\end{array}$ & $\begin{array}{l}3.076 \\
647.3 \\
1.778\end{array}$ & $\begin{array}{l}3.270 \\
682.8 \\
1.852\end{array}$ & $\begin{array}{l}3.481 \\
718.5 \\
1.926\end{array}$ & $\begin{array}{l}3.704 \\
754.2 \\
1.997\end{array}$ & $\begin{array}{l}3.934 \\
789.7 \\
2.067\end{array}$ & $\begin{array}{l}0.168 \\
825.0 \\
2.135\end{array}$ & $\begin{array}{l}4.403 \\
85.9 .9 \\
2.201\end{array}$ & $\begin{array}{l}4.637 \\
894.5 \\
2.265\end{array}$ & $\begin{array}{l}4.869 \\
928.9 \\
2.328\end{array}$ \\
\hline
\end{tabular}


Table 12 (Continued)

Temperature range: 180 to $280^{\circ} \mathrm{C}$

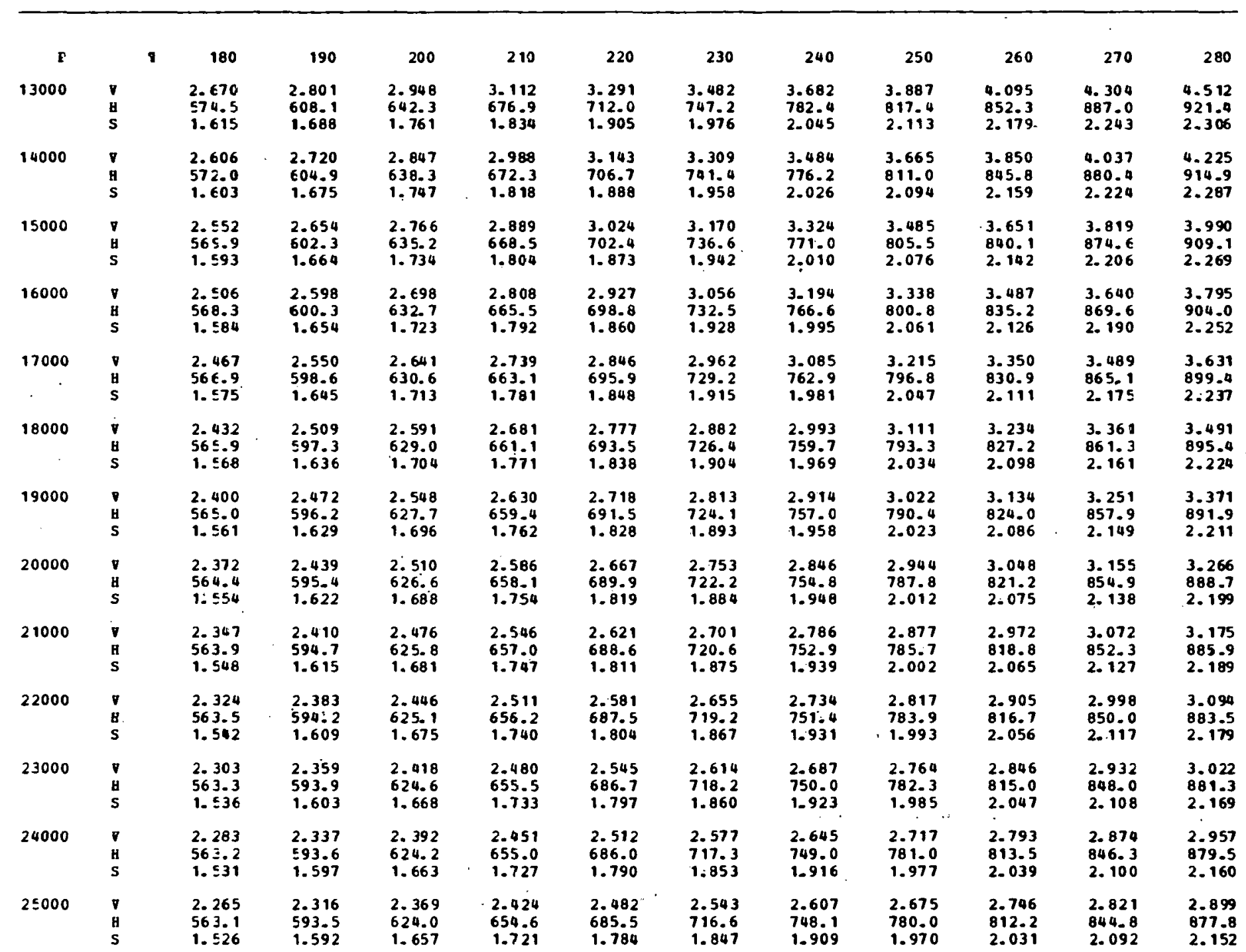


Table 12 (Continued)

Temperature range: 290 to $390^{\circ} \mathrm{C}$

\begin{tabular}{|c|c|c|c|c|c|c|c|c|c|c|c|c|}
\hline P & $\mathbf{T}$ & 290 & 300 & 310 & 320 & 330 & 340 & 350 & 360 & 370 & 380 & 390 \\
\hline 5 & $\begin{array}{l}\mathbf{V} \\
\mathrm{H} \\
\mathrm{S}\end{array}$ & $\begin{array}{r}16110 . \\
1081 . \\
3.666\end{array}$ & $\begin{array}{c}16400 \\
1109 \\
3.715\end{array}$ & $\begin{array}{r}16680 \\
1138 . \\
3.764\end{array}$ & $\begin{array}{c}16370 \\
1267 \\
3.813\end{array}$ & $\begin{array}{l}17250 . \\
1196 . \\
3.862\end{array}$ & $\begin{array}{r}17540 . \\
1225 . \\
3.910\end{array}$ & $\begin{array}{c}17830 \\
1255 \\
3.958\end{array}$ & $\begin{array}{r}18110 \\
1285 \\
4.006\end{array}$ & $\begin{array}{c}18400 \\
1315 \\
4.054\end{array}$ & $\begin{array}{r}18690 \\
1346 \\
4.101\end{array}$ & $\begin{array}{c}18970 . \\
1377 . \\
4.148\end{array}$ \\
\hline 50 & $\begin{array}{l}\nabla \\
\mathbf{y} \\
\mathbf{S}\end{array}$ & $\begin{array}{l}1610 . \\
1081 . \\
3.336\end{array}$ & $\begin{array}{l}1640 . \\
1109 . \\
3.385\end{array}$ & $\begin{array}{l}1670 . \\
1137 . \\
3.435\end{array}$ & $\begin{array}{l}1590 . \\
1166 . \\
3.483\end{array}$ & $\begin{array}{l}1720 . \\
1195 . \\
3.532\end{array}$ & $\begin{array}{l}1750 \\
1225 \\
3.581\end{array}$ & $\begin{array}{l}1780 \\
1254 \\
3.629\end{array}$ & $\begin{array}{l}1810 \\
1285 \\
3.677\end{array}$ & $\begin{array}{l}1840 . \\
1315 . \\
3.724\end{array}$ & $\begin{array}{l}1870 . \\
1346 . \\
3.772\end{array}$ & $\begin{array}{l}1900 . \\
1377 . \\
3.819\end{array}$ \\
\hline 100 & $\begin{array}{l}V \\
H \\
S\end{array}$ & $\begin{array}{l}80 \vdots .1 \\
1080 . \\
3.236\end{array}$ & $\begin{array}{l}677.6 \\
1: 08 . \\
3.286\end{array}$ & $\begin{array}{l}832.0 \\
1137 . \\
3.335\end{array}$ & $\begin{array}{l}816.4 \\
1166 . \\
3.384\end{array}$ & $\begin{array}{l}860.8 \\
1195 . \\
3.433\end{array}$ & $\begin{array}{l}875.2 \\
1224 . \\
3.481\end{array}$ & $\begin{array}{l}889.6 \\
1254 . \\
3.529\end{array}$ & $\begin{array}{l}904.0 \\
1284 . \\
3.577\end{array}$ & $\begin{array}{l}918.4 \\
1.315 . \\
3.625\end{array}$ & $\begin{array}{l}932.8 \\
1345 . \\
3.672\end{array}$ & $\begin{array}{l}947.1 \\
1376 . \\
3.719\end{array}$ \\
\hline 101.33 & $\begin{array}{l}7 \\
8 \\
5\end{array}$ & $\begin{array}{l}792.6 \\
1080 \\
3.234\end{array}$ & $\begin{array}{l}806.8 \\
1.08 \\
3.286\end{array}$ & $\begin{array}{l}821.0 \\
1137 . \\
3.333\end{array}$ & $\begin{array}{l}8.35 .3 \\
1166 . \\
3.382\end{array}$ & $\begin{array}{l}849.5 \\
1195 . \\
3.431\end{array}$ & $\begin{array}{l}863.7 \\
1224 \\
3.479\end{array}$ & $\begin{array}{l}877.9 \\
1254 . \\
3.527\end{array}$ & $\begin{array}{l}892.1 \\
1289 . \\
3.575\end{array}$ & $\begin{array}{l}906.3 \\
1315 . \\
3.623\end{array}$ & $\begin{array}{l}920.5 \\
1305 . \\
3.670\end{array}$ & $\begin{array}{l}934.7 \\
1376 . \\
3.717\end{array}$ \\
\hline 200 & $\begin{array}{l}\mathbf{V} \\
\mathrm{H} \\
\mathrm{S}\end{array}$ & $\begin{array}{l}400.3 \\
1079 . \\
3.136\end{array}$ & $\begin{array}{l}4 C .7 .6 \\
1.08 . \\
3.185\end{array}$ & $\begin{array}{l}414.9 \\
1136 . \\
3.235\end{array}$ & $\begin{array}{l}4.22 .1 \\
1165 . \\
3.284\end{array}$ & $\begin{array}{l}429.4 \\
1194 . \\
3.332\end{array}$ & $\begin{array}{l}436.6 \\
1224 . \\
3.381\end{array}$ & $\begin{array}{l}443.5 \\
1253 . \\
3.429\end{array}$ & $\begin{array}{l}451.1 \\
1283 . \\
3.477\end{array}$ & $\begin{array}{l}258.4 \\
1314 . \\
3.525\end{array}$ & $\begin{array}{l}465.6 \\
1345 . \\
3.572\end{array}$ & $\begin{array}{l}472.8 \\
1376 . \\
3.619\end{array}$ \\
\hline 300 & $\begin{array}{l}7 \\
4 \\
5\end{array}$ & $\begin{array}{l}266.1 \\
1079 . \\
3.077\end{array}$ & $\begin{array}{l}2: 1.0 \\
1: 07 . \\
3.126\end{array}$ & $\begin{array}{l}275.8 \\
1135 . \\
3.176\end{array}$ & $\begin{array}{l}230.7 \\
1164 . \\
3.225\end{array}$ & $\begin{array}{l}285.6 \\
1193 . \\
3.273\end{array}$ & $\begin{array}{l}290.5 \\
1223 . \\
3.322\end{array}$ & $\begin{array}{l}295.3 \\
1253 . \\
3.370\end{array}$ & $\begin{array}{l}300.2 \\
1283 . \\
3.418\end{array}$ & $\begin{array}{l}305.0 \\
1313: \\
3.466\end{array}$ & $\begin{array}{l}309.9 \\
1344 . \\
3.513\end{array}$ & $\begin{array}{l}314.7 \\
1375 . \\
3.561\end{array}$ \\
\hline 400 & $\begin{array}{l}y \\
\text { a } \\
\text { S }\end{array}$ & $\begin{array}{l}199.0 \\
1078 . \\
3.034\end{array}$ & $\begin{array}{l}262.6 \\
1106 . \\
3.084\end{array}$ & $\begin{array}{l}206.3 \\
1134 . \\
3.133\end{array}$ & $\begin{array}{l}210.0 \\
1163 . \\
3.182\end{array}$ & $\begin{array}{l}213.7 \\
1192 . \\
3.231\end{array}$ & $\begin{array}{l}217.4 \\
1222 . \\
3.280\end{array}$ & $\begin{array}{l}221.0 \\
1252 . \\
3.328\end{array}$ & $\begin{array}{l}224.7 \\
1282 . \\
3.376\end{array}$ & $\begin{array}{l}228.4 \\
1313 . \\
3.424\end{array}$ & $\begin{array}{l}232.0 \\
1343 . \\
3.472\end{array}$ & $\begin{array}{l}235.7 \\
1374 . \\
3.519\end{array}$ \\
\hline 500 & $\begin{array}{l}\nabla \\
H \\
S\end{array}$ & $\begin{array}{l}158.7 \\
1077 \\
3.001\end{array}$ & $\begin{array}{l}1 \in 9.7 \\
1105 . \\
3.051\end{array}$ & $\begin{array}{l}164.6 \\
1134 . \\
3.100\end{array}$ & $\begin{array}{l}167.6 \\
1162 . \\
3.150\end{array}$ & $\begin{array}{l}170.6 \\
1192 . \\
3.198\end{array}$ & $\begin{array}{l}173.5 \\
1221 . \\
3.247\end{array}$ & $\begin{array}{l}176.5 \\
1251 . \\
3.295\end{array}$ & $\begin{array}{l}179.4 \\
1281 . \\
3.343\end{array}$ & $\begin{array}{l}182.4 \\
1312 . \\
3.391\end{array}$ & $\begin{array}{l}185.3 \\
1343 . \\
3.439\end{array}$ & $\begin{array}{l}188.2 \\
1374 . \\
3.486\end{array}$ \\
\hline 600 & $\begin{array}{l}\mathbf{V} \\
\mathbf{H} \\
\mathbf{S}\end{array}$ & $\begin{array}{l}131.8 \\
1076 . \\
2.974\end{array}$ & $\begin{array}{l}134.3 \\
1104 . \\
3.024\end{array}$ & $\begin{array}{l}136.8 \\
1133 . \\
3.073\end{array}$ & $\begin{array}{l}1 \geqslant 9.3 \\
1: 62 . \\
3.122\end{array}$ & $\begin{array}{l}143.8 \\
1191 . \\
3.171\end{array}$ & $\begin{array}{l}144.3 \\
1220 . \\
3.220\end{array}$ & $\begin{array}{l}146.8 \\
1250 . \\
3.268\end{array}$ & $\begin{array}{l}149.2 \\
1281 . \\
3.317\end{array}$ & $\begin{array}{l}151.7 \\
1311 . \\
3.364\end{array}$ & $\begin{array}{l}154.2 \\
.1342 . \\
3.412\end{array}$ & $\begin{array}{r}156.6 \\
1373 . \\
-3.459\end{array}$ \\
\hline 700 & $\begin{array}{l}7 \\
1 \\
S\end{array}$ & $\begin{array}{l}112 . t \\
1075 . \\
2.951\end{array}$ & $\begin{array}{l}114.8 \\
103 . \\
3.001\end{array}$ & $\begin{array}{l}117.0 \\
1132 . \\
3.050\end{array}$ & $\begin{array}{l}1.9 .1 \\
1.61 . \\
3.099\end{array}$ & $\begin{array}{l}121.3 \\
1190 . \\
3.148\end{array}$ & $\begin{array}{l}123.4 \\
1220 . \\
3.197\end{array}$ & $\begin{array}{l}125.5 \\
1250 . \\
3.245\end{array}$ & $\begin{array}{l}127.7 \\
1280 . \\
3.294\end{array}$ & $\begin{array}{l}129.8 \\
1310= \\
3.341\end{array}$ & $\begin{array}{l}131.9 \\
.1341 . \\
3.389\end{array}$ & $\begin{array}{l}134.0 \\
1372 . \\
3.436\end{array}$ \\
\hline 300 & $\begin{array}{l}8 \\
\text { H } \\
\text { S }\end{array}$ & $\begin{array}{l}98.26 \\
1074 . \\
2.930\end{array}$ & $\begin{array}{l}100.2 \\
1002 . \\
2.980\end{array}$ & $\begin{array}{l}102.1 \\
1131 . \\
3.030\end{array}$ & $\begin{array}{l}164.0 \\
1160 . \\
3.079\end{array}$ & $\begin{array}{l}105.9 \\
1189 . \\
3.128\end{array}$ & $\begin{array}{l}107.7 \\
1219 . \\
3.177\end{array}$ & $\begin{array}{l}109.6 \\
1249 . \\
3.225\end{array}$ & $\begin{array}{l}111.5 \\
1279 . \\
3.274\end{array}$ & $\begin{array}{l}113.4 \\
1310 . \\
3.322\end{array}$ & $\begin{array}{l}115.2 \\
1341 . \\
3.369\end{array}$ & $\begin{array}{l}117.1 \\
1372 . \\
3.417\end{array}$ \\
\hline 800 & $\begin{array}{l}7 \\
H \\
5\end{array}$ & $\begin{array}{l}87.07 \\
1073 . \\
2.912\end{array}$ & $\begin{array}{l}88.78 \\
1101 . \\
2.962\end{array}$ & $\begin{array}{l}90.48 \\
1130 . \\
3.012\end{array}$ & $\begin{array}{l}92.18 \\
1159 . \\
3.061\end{array}$ & $\begin{array}{l}93.87 \\
1188 . \\
3.110\end{array}$ & $\begin{array}{l}95.56 \\
1218 . \\
3.159\end{array}$ & $\begin{array}{l}97.24 \\
1248 . \\
3.208\end{array}$ & $\begin{array}{l}98.92 \\
1278 . \\
3.256\end{array}$ & $\begin{array}{l}100.6 \\
1309 . \\
3.304\end{array}$ & $\begin{array}{l}102.3 \\
1340 . \\
3.352\end{array}$ & $\begin{array}{l}103.9 \\
1371 . \\
3: 399\end{array}$ \\
\hline 1000 & $\begin{array}{l}\text { Y } \\
H \\
\text { S }\end{array}$ & $\begin{array}{l}78.12 \\
1072 . \\
2.896\end{array}$ & $\begin{array}{l}79.67 \\
1100 . \\
2.346\end{array}$ & $\begin{array}{l}81.21 \\
1 \pm 29 . \\
2.996\end{array}$ & $\begin{array}{l}8=75 \\
1158 . \\
3.045\end{array}$ & $\begin{array}{l}84.29 \\
1188 . \\
3.094\end{array}$ & $\begin{array}{l}85.82 \\
1217 . \\
3.143\end{array}$ & $\begin{array}{l}87.34 \\
1247 \\
3.192\end{array}$ & $\begin{array}{l}88.86 \\
1278 . \\
3.240\end{array}$ & $\begin{array}{l}90.38 \\
308 . \\
3.288\end{array}$ & $\begin{array}{l}91.89 \\
1339 . \\
3.336\end{array}$ & $\begin{array}{l}93.40 \\
1370 . \\
3.383\end{array}$ \\
\hline
\end{tabular}


Table 12 (Continued)

Temperature range: 290 to $390^{\circ} \mathrm{C}$

\begin{tabular}{|c|c|c|c|c|c|c|c|c|c|c|c|c|}
\hline $\mathbf{P}$ & $\mathbf{T}$ & 290 & 300 & 310 & 320 & 330 & 340 & 350 & 360 & 370 & 380 & 390 \\
\hline 1200 & $\begin{array}{l}\mathrm{Y} \\
\mathrm{H} \\
\mathrm{H}\end{array}$ & $\begin{array}{l}64.69 \\
1070 \\
2.867\end{array}$ & $\begin{array}{l}66.01 \\
1099 . \\
2.918\end{array}$ & $\begin{array}{l}67.31 \\
1127 . \\
2.967\end{array}$ & $\begin{array}{l}68.62 \\
1157 \\
3.017\end{array}$ & $\begin{array}{l}69.91 \\
1186 . \\
3.066\end{array}$ & $\begin{array}{l}71.20 \\
1216 . \\
3.115\end{array}$ & $\begin{array}{l}72.49 \\
1246 . \\
3.969\end{array}$ & $\begin{array}{l}73.77 \\
1276 . \\
3.212\end{array}$ & $\begin{array}{l}75.05 \\
1307 . \\
3.260\end{array}$ & $\begin{array}{l}76.33 \\
1338_{-} \\
3.308\end{array}$ & $\begin{array}{l}77.60 \\
1369 \\
3.355\end{array}$ \\
\hline 1400 & $\begin{array}{l}8 \\
\mathrm{H} \\
\mathrm{s}\end{array}$ & $\begin{array}{l}55.10 \\
1068 . \\
2.843\end{array}$ & $\begin{array}{l}56.25 \\
1097 . \\
2.893\end{array}$ & $\begin{array}{l}57.39 \\
1126 . \\
2.943\end{array}$ & $\begin{array}{l}58.52 \\
1155 . \\
2.993\end{array}$ & $\begin{array}{l}59.65 \\
1184 . \\
3.042\end{array}$ & $\begin{array}{l}60.77 \\
1274 . \\
3.091\end{array}$ & $\begin{array}{l}61.89 \\
1244 . \\
3.100\end{array}$ & $\begin{array}{l}63.00 \\
1275 \\
3.188\end{array}$ & $\begin{array}{l}64.11 \\
1305 . \\
3.236\end{array}$ & $\begin{array}{l}65.22 \\
1336 . \\
3.284\end{array}$ & $\begin{array}{l}66.32 \\
3368 \\
3.332\end{array}$ \\
\hline 1600 & $\begin{array}{l}\text { : } \\
\text { B } \\
\text { s }\end{array}$ & $\begin{array}{l}97.91 \\
16 \in 6 . \\
2.821\end{array}$ & $\begin{array}{l}4693 \\
1095 . \\
2.872\end{array}$ & $\begin{array}{l}49.94 \\
1124 . \\
2.922\end{array}$ & $\begin{array}{l}50.95 \\
1153 \\
2.972\end{array}$ & $\begin{array}{l}51.95 \\
1183 . \\
3.02 .1\end{array}$ & $\begin{array}{l}52.94 \\
1213 \\
3.070\end{array}$ & $\begin{array}{l}53.94 \\
1243 . \\
3.119\end{array}$ & $\begin{array}{l}54.92 \\
1273 . \\
3.167\end{array}$ & $\begin{array}{l}55.90 \\
1304 \\
3.216\end{array}$ & $\begin{array}{l}56.88 \\
1335 . \\
3.263\end{array}$ & $\begin{array}{l}57.86 \\
1366 \\
3.311\end{array}$ \\
\hline 1800 & $\begin{array}{l}\text { H } \\
\text { H } \\
\text { S }\end{array}$ & $\begin{array}{l}4.32 \\
10 \epsilon 4 \\
2.802\end{array}$ & $\begin{array}{l}43.24 \\
1093 \\
2.852\end{array}$ & 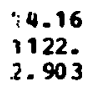 & $\begin{array}{l}45.06 \\
1151 . \\
2.952\end{array}$ & $\begin{array}{l}95.96 \\
1181 . \\
3.002\end{array}$ & $\begin{array}{l}46.86 \\
1211 . \\
3.05 i\end{array}$ & $\begin{array}{l}47.75 \\
1241 . \\
3.100\end{array}$ & $\begin{array}{l}48.64 \\
1272 . \\
3.149\end{array}$ & $\begin{array}{l}49.52 \\
1303 \\
3.197\end{array}$ & $\begin{array}{l}50.40 \\
1334 . \\
3.245\end{array}$ & $\begin{array}{l}51.28 \\
165 . \\
3.293\end{array}$ \\
\hline 2000 & $\begin{array}{l}7 \\
\mathrm{H} \\
\mathrm{S}\end{array}$ & $\begin{array}{l}37.85 \\
10 \epsilon 2 . \\
2.784\end{array}$ & $\begin{array}{l}38.69 \\
1091 . \\
2.835\end{array}$ & $\begin{array}{l}39.53 \\
2.20 . \\
2.885\end{array}$ & $\begin{array}{l}40.35 \\
1150 . \\
2.935\end{array}$ & $\begin{array}{l}41.18 \\
1179 . \\
2.985\end{array}$ & $\begin{array}{l}42.00 \\
1209 \\
3.034\end{array}$ & $\begin{array}{l}42.81 \\
1240 . \\
3.083\end{array}$ & $\begin{array}{l}43.62 \\
1270 \\
3.132\end{array}$ & $\begin{array}{l}44.42 \\
1301 . \\
3.180\end{array}$ & $\begin{array}{l}45.22 \\
1332 . \\
3.228\end{array}$ & $\begin{array}{l}46.02 \\
1364 . \\
3.276\end{array}$ \\
\hline 2100 & $\begin{array}{l}\text { H } \\
\text { H } \\
5\end{array}$ & $\begin{array}{l}35.93 \\
1061 . \\
2.776\end{array}$ & $\begin{array}{l}36.74 \\
1090 \\
2.827\end{array}$ & $\begin{array}{l}37.54 \\
1119 \\
2.877\end{array}$ & $\begin{array}{l}38.34 \\
1149 . \\
2.927\end{array}$ & $\begin{array}{l}39.13 \\
1179 \\
2.977\end{array}$ & $\begin{array}{l}39.91 \\
1209 . \\
3.026\end{array}$ & $\begin{array}{l}40.69 \\
1239 . \\
3.075\end{array}$ & $\begin{array}{l}41.46 \\
1270 \\
3.124\end{array}$ & $\begin{array}{l}42.23 \\
1300 \\
3.172\end{array}$ & $\begin{array}{l}\begin{array}{l}43.00 \\
1332 \\
3.220\end{array} \\
3.220\end{array}$ & $\begin{array}{l}43.76 \\
1363 . \\
3.268\end{array}$ \\
\hline 2200 & $\begin{array}{l}\mathrm{I} \\
\mathrm{u} \\
\mathrm{s}\end{array}$ & $\begin{array}{l}34.19 \\
1061 . \\
2.768\end{array}$ & $\begin{array}{l}34.97 \\
1089 . \\
2.819\end{array}$ & $\begin{array}{l}35.74 \\
1119 . \\
2.869\end{array}$ & $\begin{array}{l}36.50 \\
1148 . \\
2.919\end{array}$ & $\begin{array}{l}37.26 \\
1178 . \\
2.969\end{array}$ & $\begin{array}{l}38.02 \\
1208 \\
3.019\end{array}$ & $\begin{array}{l}38.76 \\
1238 . \\
3.068\end{array}$ & $\begin{array}{l}39.51 \\
1269 . \\
3.116\end{array}$ & $\begin{array}{l}40.25 \\
1300 . \\
3.165\end{array}$ & $\begin{array}{l}\begin{array}{l}0.98 \\
1331 . \\
3.213\end{array} \\
3.2\end{array}$ & $\begin{array}{l}41.72 \\
1362 . \\
3.261\end{array}$ \\
\hline 2300 & $\begin{array}{l}\text { H } \\
\text { H } \\
\text { S }\end{array}$ & $\begin{array}{l}32.60 \\
1060 . \\
2.760\end{array}$ & $\begin{array}{l}33.35 \\
1088 . \\
2.811\end{array}$ & $\begin{array}{l}34.09 \\
1118 . \\
2.862\end{array}$ & $\begin{array}{l}34.83 \\
1147 \\
2.912\end{array}$ & $\begin{array}{l}35.56 \\
1177 . \\
2.962\end{array}$ & $\begin{array}{l}36.29 \\
1207 \\
3.011\end{array}$ & $\begin{array}{l}37.01 \\
1237 . \\
3.060\end{array}$ & $\begin{array}{l}37.72 \\
1268 . \\
3.109\end{array}$ & $\begin{array}{l}38.43 \\
1299 . \\
3.158\end{array}$ & $\begin{array}{l}39.14 \\
1330 . \\
3.20 E\end{array}$ & $\begin{array}{l}35.85 \\
1362 . \\
3.254\end{array}$ \\
\hline 2400 & $\begin{array}{l}\text { H } \\
\text { H } \\
\text { S }\end{array}$ & $\begin{array}{l}31.16 \\
1059 . \\
2.7 \leq 3\end{array}$ & $\begin{array}{l}31.87 \\
1087 \\
2.804\end{array}$ & $\begin{array}{l}32.59 \\
1117 . \\
2.854\end{array}$ & $\begin{array}{l}33.30 \\
1146 . \\
2.905\end{array}$ & $\begin{array}{l}34.00 \\
1176 . \\
2.955\end{array}$ & $\begin{array}{l}34.70 \\
1206 \\
3.004\end{array}$ & $\begin{array}{l}35-40 \\
1237 . \\
3.053\end{array}$ & $\begin{array}{l}36.09 \\
1267 \\
3.102\end{array}$ & $\begin{array}{l}36.77 \\
1298 \\
3.15 i\end{array}$ & $\begin{array}{l}37.45 \\
1330 . \\
3.199\end{array}$ & $\begin{array}{l}38.13 \\
1369 \\
3.247 \\
3.247\end{array}$ \\
\hline zंsoo & $\begin{array}{l}\mathrm{D} \\
\mathrm{H} \\
\mathrm{S}\end{array}$ & $\begin{array}{l}29.80 \\
3058 . \\
2.746\end{array}$ & $\begin{array}{l}30.50 \\
1087 \\
2.797\end{array}$ & $\begin{array}{l}31.20 \\
1116 . \\
2.847\end{array}$ & $\begin{array}{l}31.89 \\
1145 . \\
2.898\end{array}$ & $\begin{array}{l}32.57 \\
1175 . \\
2.948\end{array}$ & $\begin{array}{l}33.24 \\
1205 \\
2.997\end{array}$ & $\begin{array}{l}33.92 \\
1236 . \\
3.046\end{array}$ & $\begin{array}{l}34.58 \\
1267 . \\
3.095\end{array}$ & $\begin{array}{l}35.24 \\
1298 . \\
3.144\end{array}$ & $\begin{array}{r}35.90 \\
-1329 . \\
3.192\end{array}$ & $\begin{array}{l}36.56 \\
1360 . \\
3.240\end{array}$ \\
\hline 2600 & $\begin{array}{l}\mathrm{V} \\
\mathrm{H} \\
\mathrm{S}\end{array}$ & $\begin{array}{l}28.56 \\
1057 \\
2.739\end{array}$ & $\begin{array}{l}29.24 \\
1086 \\
2.790\end{array}$ & $\begin{array}{l}29.92 \\
1115 \\
2.841\end{array}$ & $\begin{array}{l}30.58 \\
1145 \\
2.891\end{array}$ & $\begin{array}{l}31.24 \\
1174 \\
2.941\end{array}$ & $\begin{array}{l}31.90 \\
1205 . \\
2.99 .1\end{array}$ & $\begin{array}{l}32.55 \\
1235 . \\
3.040\end{array}$ & $\begin{array}{l}33.19 \\
1266 . \\
3.089\end{array}$ & $\begin{array}{l}33.83 \\
1297 . \\
3.137\end{array}$ & $\begin{array}{l}34.47 \\
1328 . \\
3.186\end{array}$ & $\begin{array}{l}35.10 \\
1360 . \\
3.239\end{array}$ \\
\hline 2700 & $\begin{array}{l}Y \\
\text { H } \\
S\end{array}$ & $\begin{array}{l}27.42 \\
1056 . \\
2.732\end{array}$ & $\begin{array}{l}28.08 \\
1085 \\
2.783\end{array}$ & $\begin{array}{l}28.73 \\
1114 . \\
2.834\end{array}$ & $\begin{array}{l}29.38 \\
1144 . \\
2.884\end{array}$ & $\begin{array}{l}30.02 \\
1174 . \\
2.934\end{array}$ & $\begin{array}{l}30.65 \\
1204 \\
2.984\end{array}$ & $\begin{array}{l}31.28 \\
1234 . \\
3.034\end{array}$ & $\begin{array}{l}31.91 \\
1265 . \\
3.082\end{array}$ & $\begin{array}{l}32.53 \\
1296 \\
3.13 i\end{array}$ & $\begin{array}{l}33.14 \\
1327 . \\
3.179\end{array}$ & $\begin{array}{l}33.76 \\
1359 . \\
3.227\end{array}$ \\
\hline 2800 & $\begin{array}{l}\text { V } \\
\text { H } \\
\text { S }\end{array}$ & $\begin{array}{l}26.35 \\
1055 . \\
2.725\end{array}$ & $\begin{array}{l}27.00 \\
1084 \\
2.777\end{array}$ & $\begin{array}{l}27.63 \\
1113 . \\
2.828\end{array}$ & $\begin{array}{l}28.26 \\
1143 . \\
2.878\end{array}$ & $\begin{array}{l}28.88 \\
1173 . \\
2.928\end{array}$ & $\begin{array}{l}29.50 \\
1203 . \\
2.978\end{array}$ & $\begin{array}{l}30.11 \\
1234 . \\
3.027\end{array}$ & $\begin{array}{l}30.71 \\
1264 . \\
3.076\end{array}$ & $\begin{array}{l}31.32 \\
1295 . \\
3.125\end{array}$ & $\begin{array}{l}31.91 \\
1327 . \\
3.173\end{array}$ & $\begin{array}{l}32.51 \\
1358 \\
3.221\end{array}$ \\
\hline
\end{tabular}


Table 12 (Continued)

Temperature range: 290 to $390^{\circ} \mathrm{F}$

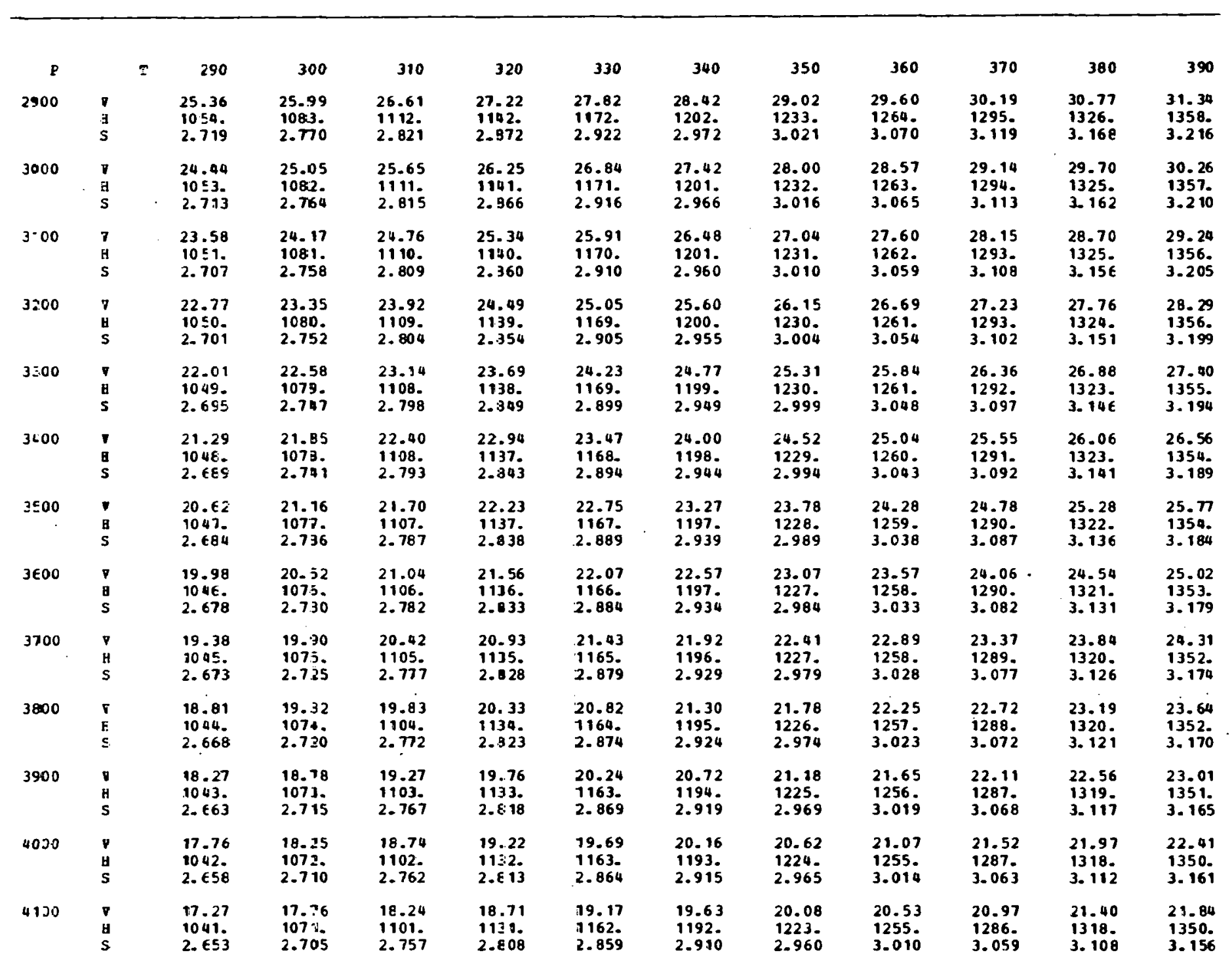


Table 12 (Continued)

Temperature range: 290 to $390^{\circ} \mathrm{C}$

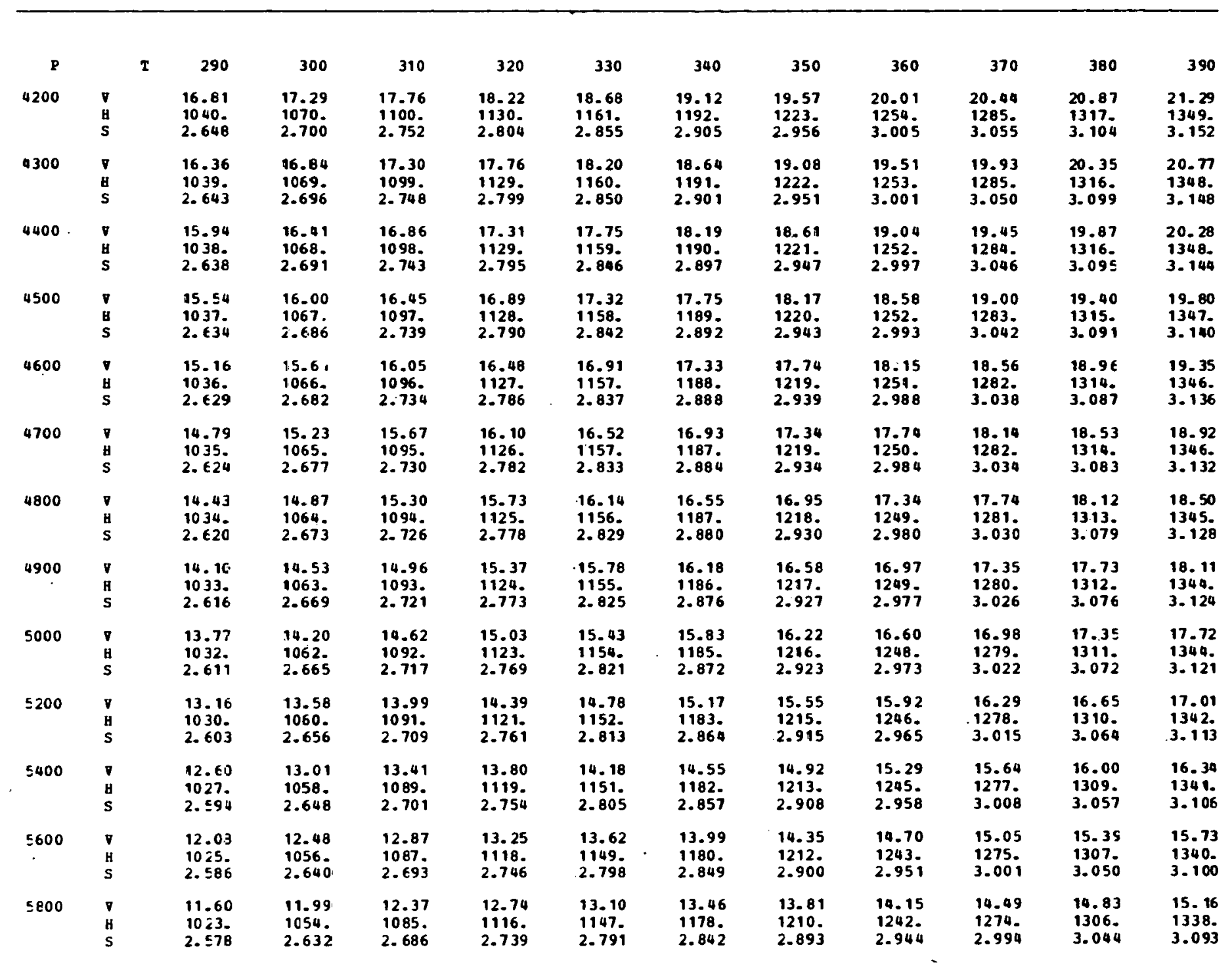


Table 12 (Continued)

Temperature range: 290 to $390^{\circ} \mathrm{C}$

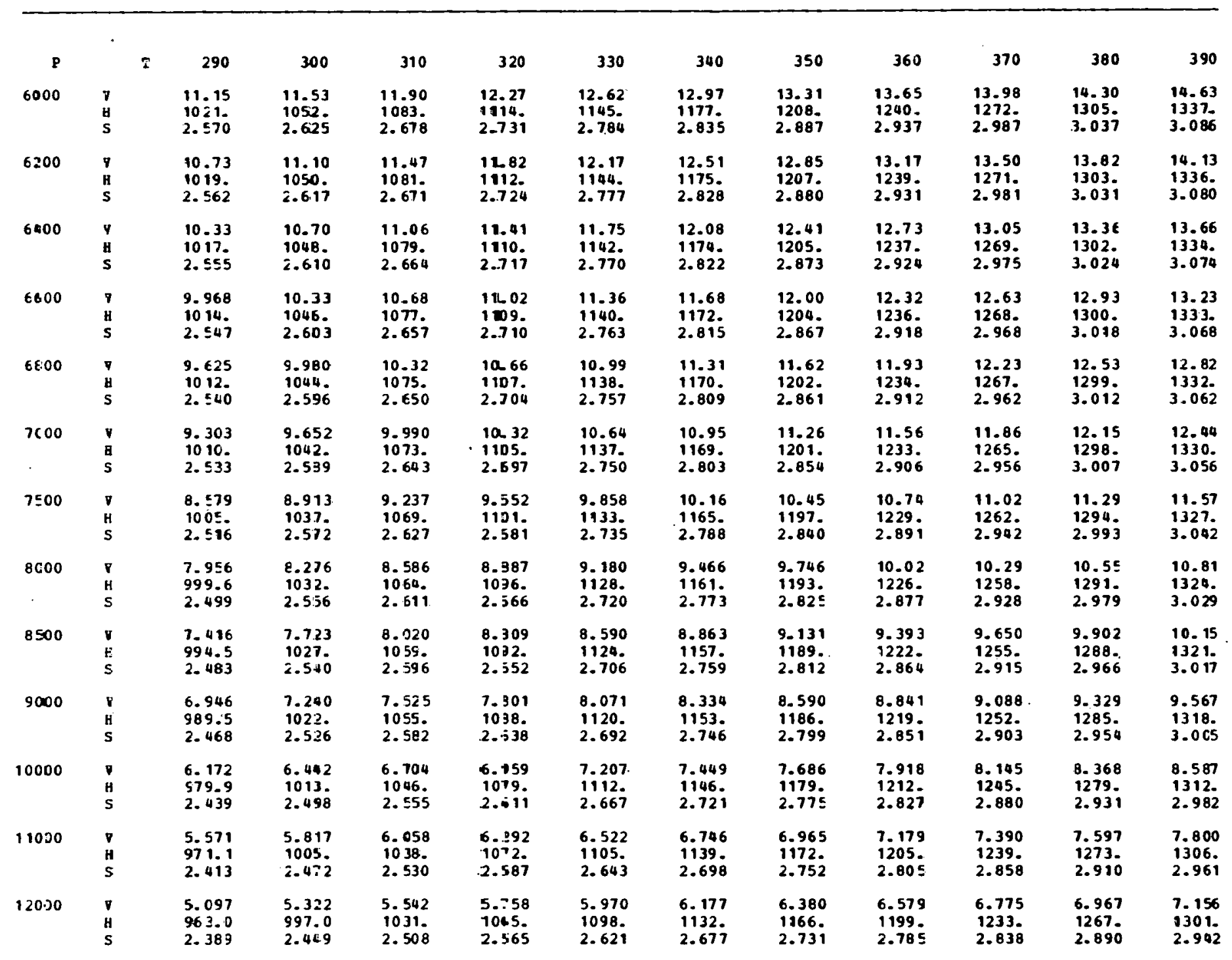


Table 12 (Continued)

Temperature range: 290 to $390^{\circ} \mathrm{C}$

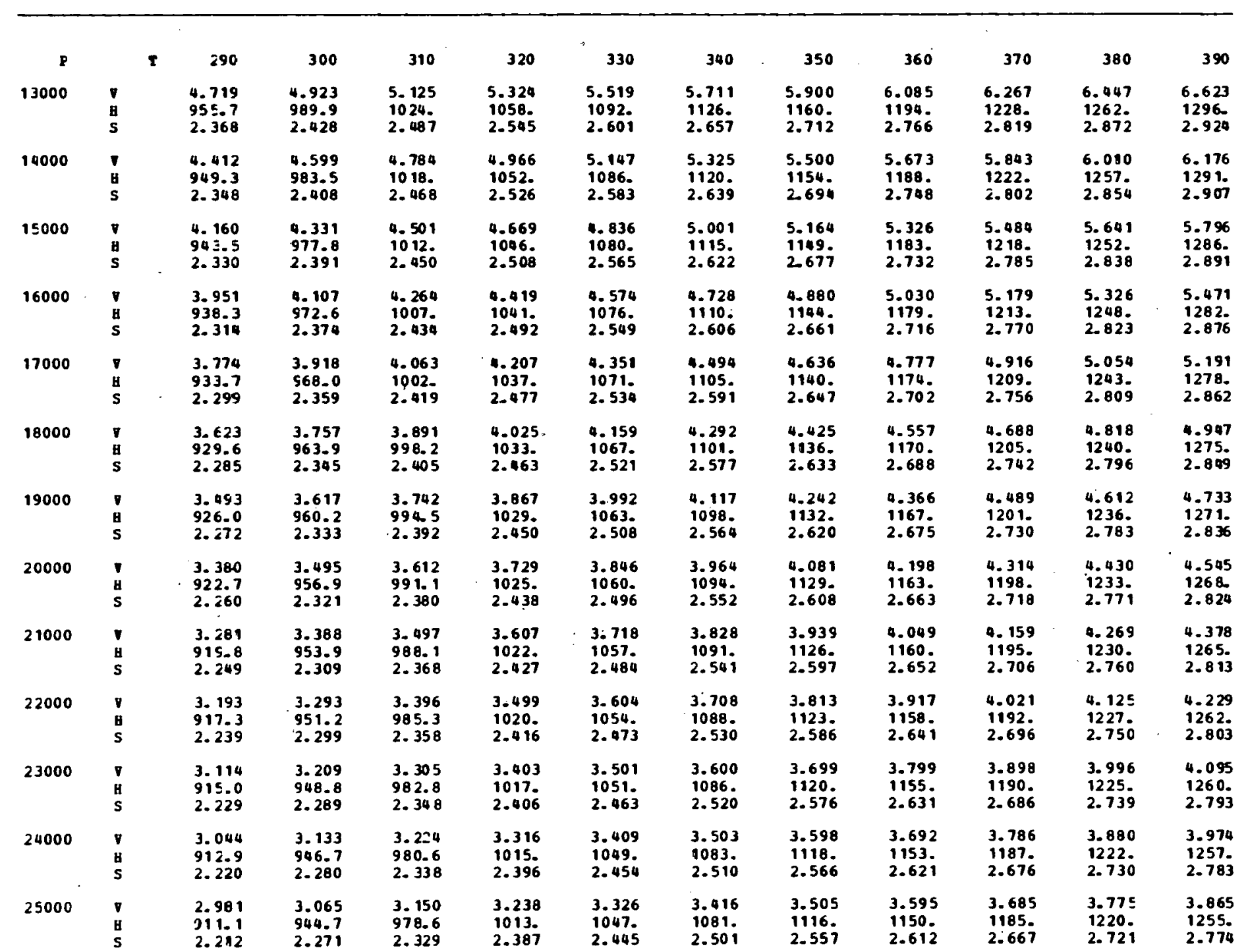


Table 12 (Continued)

Temperature range: 400 to $500^{\circ} \mathrm{C}$

\begin{tabular}{|c|c|c|c|c|c|c|c|c|c|c|c|c|}
\hline P & & 400 & 410 & 420 & 430 & 440 & 450 & 460 & 470 & 480 & 490 & 500 \\
\hline 5 & $\begin{array}{l}\nabla \\
t \\
S\end{array}$ & $\begin{array}{r}09260 . \\
14 \mathrm{CB} \\
4.195\end{array}$ & $\begin{array}{l}1954 C \\
144 C \\
4.242\end{array}$ & $\begin{array}{r}19830 . \\
1472 . \\
4.288\end{array}$ & $\begin{array}{r}20120 \\
1504 \\
4.335\end{array}$ & $\begin{array}{r}20400 . \\
1537 . \\
4.380\end{array}$ & $\begin{array}{r}20690 \\
1569 \\
4.426\end{array}$ & $\begin{array}{r}20970 \\
1602 \\
4.471\end{array}$ & $\begin{array}{r}21260 . \\
1636 \\
4.517\end{array}$ & $\begin{array}{r}21550 \\
1669 \\
4.562\end{array}$ & $\begin{array}{r}21830 . \\
1703 . \\
4.606\end{array}$ & $\begin{array}{r}22120 . \\
1737 \\
4.651\end{array}$ \\
\hline so & $\begin{array}{l}\nabla \\
H \\
S\end{array}$ & $\begin{array}{l}1920 . \\
14 C 8 . \\
3.866\end{array}$ & $\begin{array}{l}195 C . \\
144 C . \\
3.912\end{array}$ & $\begin{array}{l}1980 . \\
1472 . \\
3.959\end{array}$ & $\begin{array}{l}2010 . \\
1504 . \\
4.005\end{array}$ & $\begin{array}{l}2040 . \\
1536 \\
4.051\end{array}$ & $\begin{array}{l}2070 \\
1569 . \\
4.096\end{array}$ & $\begin{array}{l}2100 \\
1602 \\
4.142\end{array}$ & $\begin{array}{l}2130 . \\
1636 \\
4.187\end{array}$ & $\begin{array}{l}2150 . \\
1669 . \\
4.232\end{array}$ & $\begin{array}{l}2180 . \\
1703 \\
4.277\end{array}$ & $\begin{array}{l}2210 . \\
1737 . \\
4.321\end{array}$ \\
\hline 100 & $\begin{array}{l}\nabla \\
\text { u } \\
\text { S }\end{array}$ & $\begin{array}{l}961.5 \\
1408 . \\
3.766\end{array}$ & $\begin{array}{l}\$ 75 .-9 \\
1439 . \\
3.81\end{array}$ & $\begin{array}{l}990.3 \\
1471 . \\
3.859\end{array}$ & $\begin{array}{l}1000 \\
1504 \\
3.905\end{array}$ & $\begin{array}{l}1020 . \\
1536 . \\
3.951\end{array}$ & $\begin{array}{l}1030 \\
1569 \\
3.997\end{array}$ & $\begin{array}{l}1050 \\
1602 \\
4.042\end{array}$ & $\begin{array}{l}1060 . \\
1635 \\
4.088\end{array}$ & $\begin{array}{l}1080 . \\
1669 . \\
4.133\end{array}$ & $\begin{array}{l}1090 . \\
1703 \\
4.17 j\end{array}$ & $\begin{array}{l}1110 . \\
1737 . \\
4.222\end{array}$ \\
\hline 101.33 & $\begin{array}{l}v \\
\mathrm{H} \\
\mathrm{S}\end{array}$ & $\begin{array}{l}948.9 \\
14 C 8 . \\
3.764\end{array}$ & $\begin{array}{l}963.1 \\
1439 . \\
3.8111\end{array}$ & $\begin{array}{l}977.3 \\
1471 . \\
3.857\end{array}$ & $\begin{array}{l}991.4 \\
1504 . \\
3.904\end{array}$ & $\begin{array}{l}1010 . \\
1536 \\
3.949\end{array}$ & $\begin{array}{l}1020 \\
1569 \\
3.995\end{array}$ & $\begin{array}{l}1030 . \\
1602 . \\
4.041\end{array}$ & $\begin{array}{l}1050 . \\
1635 \\
4.086\end{array}$ & $\begin{array}{l}1060 . \\
1669 \\
4.131\end{array}$ & $\begin{array}{l}1080 . \\
1703 . \\
4.175\end{array}$ & $\begin{array}{l}1090 . \\
1737 . \\
4.220\end{array}$ \\
\hline 2010 & $\begin{array}{l}\nabla \\
\mathrm{H} \\
\mathrm{S}\end{array}$ & $\begin{array}{l}480.1 \\
1407 . \\
3.666\end{array}$ & $\begin{array}{l}487.3 \\
1439 . \\
3.713\end{array}$ & $\begin{array}{l}494.5 \\
1471 . \\
3.759\end{array}$ & $\begin{array}{l}501.7 \\
1503 . \\
3.806\end{array}$ & $\begin{array}{l}508.9 \\
1535 . \\
3.852\end{array}$ & $\begin{array}{l}516.2 \\
1568 . \\
3.897\end{array}$ & $\begin{array}{l}523.4 \\
1601 . \\
3.943\end{array}$ & $\begin{array}{l}530.6 \\
1635 . \\
3.988\end{array}$ & $\begin{array}{l}537.8 \\
1668 . \\
4.233\end{array}$ & $\begin{array}{l}545 . C \\
1702 . \\
4.078\end{array}$ & $\begin{array}{l}552.2 \\
1736 . \\
4.122\end{array}$ \\
\hline $3 \mathrm{CO}$ & $\begin{array}{l}y \\
y \\
s\end{array}$ & $\begin{array}{l}319.6 \\
1406 . \\
3.608\end{array}$ & $\begin{array}{l}324.0 \\
1438 . \\
3.654\end{array}$ & $\begin{array}{l}329.2 \\
1470 . \\
3.701\end{array}$ & $\begin{array}{l}334.1 \\
1502 . \\
3.747\end{array}$ & $\begin{array}{l}338.9 \\
1535 . \\
3.793\end{array}$ & $\begin{array}{l}343.8 \\
1568 . \\
3.839\end{array}$ & $\begin{array}{l}348.6 \\
1601 . \\
3.884\end{array}$ & $\begin{array}{l}353.4 \\
1634 . \\
3.929\end{array}$ & $\begin{array}{l}359.2 \\
16.58 . \\
3.374\end{array}$ & $\begin{array}{l}363.1 \\
1702 . \\
4.019\end{array}$ & $\begin{array}{l}367.9 \\
1736 . \\
4.064\end{array}$ \\
\hline 400 & $\begin{array}{l}7 \\
4 \\
5\end{array}$ & $\begin{array}{l}239.3 \\
1406 . \\
1.566\end{array}$ & $\begin{array}{l}243.3 \\
1438 \\
3.613\end{array}$ & $\begin{array}{l}246.6 \\
1470 . \\
3.659\end{array}$ & $\begin{array}{l}250.3 \\
1502 . \\
3.705\end{array}$ & $\begin{array}{l}253.9 \\
1534 \\
3.751\end{array}$ & $\begin{array}{l}257.6 \\
1567 . \\
3.797\end{array}$ & $\begin{array}{l}261.2 \\
1600 . \\
3.842\end{array}$ & $\begin{array}{l}264.8 \\
1634 . \\
3.888\end{array}$ & $\begin{array}{l}263.5 \\
1657 . \\
3.933\end{array}$ & $\begin{array}{l}272.1 \\
1701 . \\
3.977\end{array}$ & $\begin{array}{l}275.7 \\
1735 . \\
0.022\end{array}$ \\
\hline$\leq 00$ & $\begin{array}{l}\nabla \\
B \\
S\end{array}$ & $\begin{array}{l}191.2 \\
1405 \\
1.533\end{array}$ & $\begin{array}{l}194 . \\
1437 \\
3.580\end{array}$ & $\begin{array}{l}197.1 \\
1469 . \\
3.626\end{array}$ & $\begin{array}{l}200.0 \\
1501 . \\
3.673\end{array}$ & $\begin{array}{l}202.9 \\
1534 . \\
3.719\end{array}$ & $\begin{array}{l}205.8 \\
1567 . \\
3.760\end{array}$ & $\begin{array}{l}208.8 \\
1600 . \\
3.810\end{array}$ & $\begin{array}{l}211.7 \\
1633 . \\
3.855\end{array}$ & $\begin{array}{l}214.6 \\
1667 . \\
3.900\end{array}$ & $\begin{array}{l}217.5 \\
1701: \\
3.945\end{array}$ & $\begin{array}{l}220.4 \\
1735 . \\
3.989\end{array}$ \\
\hline 600 & $\begin{array}{l}\mathbf{P} \\
H \\
5\end{array}$ & $\begin{array}{l}159.1 \\
.405 \\
3.506\end{array}$ & $\begin{array}{l}161.5 \\
1436 \\
3.55 j\end{array}$ & $\begin{array}{l}164.0 \\
1468 \\
3.600\end{array}$ & $\begin{array}{l}166.5 \\
1501 . \\
3.646\end{array}$ & $\begin{array}{l}168.9 \\
1533 . \\
3.692\end{array}$ & $\begin{array}{l}171.4 \\
1566 . \\
3.738\end{array}$ & $\begin{array}{l}173.8 \\
1599 . \\
3.783\end{array}$ & $\begin{array}{l}176.2 \\
1633 . \\
3.828\end{array}$ & $\begin{array}{l}178.7 \\
1666 . \\
3.873\end{array}$ & $\begin{array}{l}181.1 \\
1700 . \\
3.918\end{array}$ & $\begin{array}{l}183.6 \\
1734 . \\
3.963\end{array}$ \\
\hline 700 & $\begin{array}{l}V \\
H \\
S\end{array}$ & \begin{tabular}{l}
136.2 \\
1404. \\
\hdashline .483
\end{tabular} & $\begin{array}{l}138.1 \\
1436 . \\
3.530\end{array}$ & $\begin{array}{l}140.4 \\
1468 \\
3.577\end{array}$ & $\begin{array}{l}142.5 \\
1500 . \\
3.623\end{array}$ & $\begin{array}{l}144.6 \\
1533 . \\
3.669\end{array}$ & $\begin{array}{l}146.7 \\
1565 . \\
3.715\end{array}$ & $\begin{array}{l}148.8 \\
1599 . \\
3.761\end{array}$ & $\begin{array}{l}150.9 \\
1632 . \\
3.806\end{array}$ & $\begin{array}{l}153.0 \\
1666 . \\
3.851\end{array}$ & $\begin{array}{l}155.1 \\
1700 . \\
3.896\end{array}$ & $\begin{array}{l}157.2 \\
1734 . \\
3.940\end{array}$ \\
\hline 801) & $\begin{array}{l}\nabla \\
H \\
S\end{array}$ & $\begin{array}{l}119.0 \\
1403 . \\
3.464\end{array}$ & $\begin{array}{l}120.8 \\
1435 . \\
3.5 \%\end{array}$ & $\begin{array}{l}122.7 \\
1467 . \\
3.557\end{array}$ & $\begin{array}{l}124.6 \\
1499 . \\
3.603\end{array}$ & $\begin{array}{l}126.4 \\
1532 . \\
3.649\end{array}$ & $\begin{array}{l}128.3 \\
1565 . \\
3.695\end{array}$ & $\begin{array}{l}130.1 \\
1598 . \\
3.741\end{array}$ & $\begin{array}{l}132.0 \\
1632 . \\
3.786\end{array}$ & $\begin{array}{l}133.8 \\
166.5 \\
3.831\end{array}$ & $\begin{array}{l}135.6 \\
1699 . \\
3.876\end{array}$ & $\begin{array}{l}137.5 \\
1733 . \\
3.921\end{array}$ \\
\hline 900 & $\begin{array}{l}\nabla \\
\mathbf{u} \\
\mathbf{S}\end{array}$ & $\begin{array}{l}10 \leq-6 \\
1403 . \\
3.446\end{array}$ & $\begin{array}{l}107 . \Sigma \\
1434_{-} \\
3.493\end{array}$ & $\begin{array}{l}108.9 \\
1466 . \\
3.540\end{array}$ & $\begin{array}{l}110.6 \\
1499 . \\
3.586\end{array}$ & $\begin{array}{l}112.2 \\
1531 . \\
3.632\end{array}$ & $\begin{array}{l}113.9 \\
1564 . \\
3.678\end{array}$ & $\begin{array}{l}115.5 \\
1598 . \\
\equiv .723\end{array}$ & $\begin{array}{l}117.2 \\
1631 . \\
\vdots .769\end{array}$ & $\begin{array}{l}11 E-8 \\
16 \in 5 . \\
3 . \varepsilon 14\end{array}$ & $\begin{array}{l}120.5 \\
1699 \\
3.859\end{array}$ & $\begin{array}{l}122.1 \\
1733 . \\
3.903\end{array}$ \\
\hline 1000 & $\begin{array}{l}7 \\
H \\
S\end{array}$ & $\begin{array}{l}94.91 \\
1402 . \\
3.430\end{array}$ & $\begin{array}{l}96.42 \\
1434 \\
3.477\end{array}$ & $\begin{array}{l}97.92 \\
1466 . \\
3.524\end{array}$ & $\begin{array}{l}99.42 \\
1498 . \\
3.570\end{array}$ & $\begin{array}{l}100.9 \\
1531 . \\
3.616\end{array}$ & $\begin{array}{l}102.4 \\
1564 . \\
3.662\end{array}$ & $\begin{array}{l}103.9 \\
1597 . \\
3.708\end{array}$ & $\begin{array}{l}105.4 \\
1630 \\
5.753\end{array}$ & $\begin{array}{l}10 \epsilon .9 \\
16 \in 4- \\
3.798\end{array}$ & $\begin{array}{l}108.9 \\
1698 . \\
3.843\end{array}$ & $\begin{array}{l}109.9 \\
1732 . \\
3.888\end{array}$ \\
\hline
\end{tabular}


Table 12 (Continued)

Temperature range: 400 to $500^{\circ} \mathrm{C}$

\begin{tabular}{|c|c|c|c|c|c|c|c|c|c|c|c|c|c|}
\hline P & & I & 400 & 410 & 420 & 430 & 440 & 450 & 460 & 470 & 480 & 490 & 500 \\
\hline 1200 & $\begin{array}{l}\nabla \\
H \\
S\end{array}$ & $\ddots$ & $\begin{array}{l}78.87 \\
140.1 . \\
3.403\end{array}$ & $\begin{array}{l}80.14 \\
1433 . \\
3.450\end{array}$ & $\begin{array}{l}81.40 \\
1465 . \\
3.496\end{array}$ & $\begin{array}{l}82.66 \\
1497 . \\
3.543\end{array}$ & $\begin{array}{l}83.92 \\
1530 . \\
3 ., 589\end{array}$ & $\begin{array}{l}85.18 \\
1563 . \\
3.635\end{array}$ & $\begin{array}{l}86.43 \\
1596 . \\
3.680\end{array}$ & $\begin{array}{l}87.68 \\
1629 . \\
3.726\end{array}$ & $\begin{array}{l}88.93 \\
1663 \\
3.771\end{array}$ & $\begin{array}{l}90.18 \\
1697 . \\
3.816\end{array}$ & $\begin{array}{l}91.43 \\
1731 . \\
3.860\end{array}$ \\
\hline 1400 & $\begin{array}{l}7 \\
8 \\
5\end{array}$ & & $\begin{array}{l}67.42 \\
1399 \\
3.379\end{array}$ & $\begin{array}{l}68.51 \\
9431 . \\
3.426\end{array}$ & $\begin{array}{l}69.61 \\
1463 . \\
3.473\end{array}$ & $\begin{array}{l}70.70 \\
1496 . \\
3.519\end{array}$ & $\begin{array}{l}71.79 \\
1529 . \\
3.566\end{array}$ & $\begin{array}{l}72.87 \\
1562 \\
3.611\end{array}$ & $\begin{array}{l}73.96 \\
1595 \\
3.657\end{array}$ & $\begin{array}{l}75.04 \\
1628 \\
3.703\end{array}$ & $\begin{array}{l}76.12 \\
1662 \\
3.748\end{array}$ & $\begin{array}{l}77.20 \\
1696 \\
3.793\end{array}$ & $\begin{array}{l}78.27 \\
1731 . \\
3.837\end{array}$ \\
\hline 1600 & $\begin{array}{l}y \\
y \\
s\end{array}$ & & $\begin{array}{l}58-83 \\
1398 . \\
3: 358\end{array}$ & $\begin{array}{l}59.80 \\
1430 . \\
3.406\end{array}$ & $\begin{array}{l}60.76 \\
1462 . \\
3.452\end{array}$ & $\begin{array}{l}61.73 \\
1095 . \\
3.499\end{array}$ & $\begin{array}{l}62.69 \\
1527 \\
3.545\end{array}$ & $\begin{array}{l}63.65 \\
1560 \\
3.591\end{array}$ & $\begin{array}{l}64.60 \\
1594 . \\
3.637\end{array}$ & $\begin{array}{l}65.56 \\
1627 . \\
3.682\end{array}$ & $\begin{array}{l}66.51 \\
1661 . \\
3.727\end{array}$ & $\begin{array}{l}67.46 \\
1695 . \\
3.772\end{array}$ & $\begin{array}{l}68.41 \\
1730 . \\
3.8 \pi\end{array}$ \\
\hline 1800 & $\begin{array}{l}\forall \\
\mathbf{H} \\
\mathbf{S}\end{array}$ & & $\begin{array}{l}52.15 \\
1397 . \\
3.340\end{array}$ & $\begin{array}{l}53.02 \\
1429 . \\
3.387\end{array}$ & $\begin{array}{l}53.89 \\
1461 . \\
3.434\end{array}$ & $\begin{array}{l}54.75 \\
1493 . \\
3.481\end{array}$ & $\begin{array}{l}55.61 \\
1526 . \\
3.527\end{array}$ & $\begin{array}{l}56.47 \\
1559 \\
3.573\end{array}$ & $\begin{array}{l}57.33 \\
1593 . \\
3.619\end{array}$ & $\begin{array}{l}58.19 \\
1626 . \\
3.664\end{array}$ & $\begin{array}{l}59.04 \\
1660 . \\
3.709\end{array}$ & $\begin{array}{l}59.89 \\
1694 . \\
3.754\end{array}$ & $\begin{array}{l}60.74 \\
1729 . \\
3.799\end{array}$ \\
\hline 2000 & $\begin{array}{l}7 \\
H \\
5\end{array}$ & & $\begin{array}{l}46.81 \\
1395 . \\
3.324\end{array}$ & $\begin{array}{l}47.60 \\
1427 \\
3.371\end{array}$ & $\begin{array}{l}48.39 \\
1460 . \\
3.418\end{array}$ & $\begin{array}{l}49.17 \\
1492 . \\
3.464\end{array}$ & $\begin{array}{l}49.96 \\
1525 . \\
3.511\end{array}$ & $\begin{array}{l}50.74 \\
1558 . \\
3.557\end{array}$ & $\begin{array}{l}51.51 \\
1592 . \\
3.602\end{array}$ & $\begin{array}{l}52.29 \\
1625 \\
3.648\end{array}$ & $\begin{array}{l}53.06 \\
1659 \\
3.693\end{array}$ & $\begin{array}{l}53.83 \\
1693 . \\
3.738\end{array}$ & $\begin{array}{l}54.60 \\
1728 . \\
3.783\end{array}$ \\
\hline 2100 & $\begin{array}{l}7 \\
B \\
S\end{array}$ & & $\begin{array}{l}44.52 \\
1395 \\
3.316\end{array}$ & $\begin{array}{l}45.28 \\
1427 \\
3.363\end{array}$ & $\begin{array}{l}46.03 \\
1459 . \\
3.410\end{array}$ & $\begin{array}{l}46.78 \\
1492 . \\
3.457\end{array}$ & $\begin{array}{l}47.53 \\
1525 . \\
3.503\end{array}$ & $\begin{array}{l}48.28 \\
1558 . \\
3.549\end{array}$ & $\begin{array}{l}49.02 \\
1591 . \\
3.595\end{array}$ & $\begin{array}{l}49.76 \\
1625 . \\
3.640\end{array}$ & $\begin{array}{l}50.50 \\
1659 . \\
3.686\end{array}$ & $\begin{array}{l}51.24 \\
1693 \\
3.731\end{array}$ & $\begin{array}{l}51.98 \\
1727 . \\
3.776\end{array}$ \\
\hline 2200 & $\begin{array}{l}7 \\
y \\
S\end{array}$ & & $\begin{array}{l}42.44 \\
1394 . \\
3.308\end{array}$ & $\begin{array}{l}13.07 \\
1426 \\
3.356\end{array}$ & $\begin{array}{l}43.89 \\
1459 \\
3.403\end{array}$ & $\begin{array}{l}44.61 \\
1491 . \\
3.449\end{array}$ & $\begin{array}{l}45.33 \\
1524 . \\
3.496\end{array}$ & $\begin{array}{l}46.04 \\
1557 . \\
3.542\end{array}$ & $\begin{array}{l}46.76 \\
1590 . \\
3.588\end{array}$ & $\begin{array}{l}97.47 \\
1624 . \\
3.633\end{array}$ & $\begin{array}{l}08.18 \\
1658 . \\
3.679\end{array}$ & $\begin{array}{l}48.88 \\
1692 . \\
3.724\end{array}$ & $\begin{array}{l}49.59 \\
1727 . \\
3.768\end{array}$ \\
\hline 2300 & $\begin{array}{l}\nabla \\
H \\
S\end{array}$ & & $\begin{array}{l}40.55 \\
1394 . \\
3.301\end{array}$ & $\begin{array}{l}11.24 \\
1426 . \\
3.348\end{array}$ & $\begin{array}{l}41.94 \\
1458 . \\
3.395\end{array}$ & $\begin{array}{l}42.63 \\
1491 . \\
3.442\end{array}$ & $\begin{array}{l}43.32 \\
1523 . \\
3.489\end{array}$ & $\begin{array}{l}44.01 \\
1557 \\
3.535\end{array}$ & $\begin{array}{l}44.69 \\
1590 \\
3.581\end{array}$ & $\begin{array}{l}45.37 \\
1624 . \\
3.626\end{array}$ & $\begin{array}{l}16.05 \\
1658 \\
3.672\end{array}$ & $\begin{array}{l}46.73 \\
1692 \\
3.717\end{array}$ & $\begin{array}{l}47.41 \\
1726 . \\
3.761\end{array}$ \\
\hline 2400 & $\begin{array}{l}\nabla \\
H \\
S\end{array}$ & & $\begin{array}{l}38.81 \\
1393 . \\
3.294\end{array}$ & $\begin{array}{l}39.48 \\
1425 . \\
3.342\end{array}$ & $\begin{array}{l}40.15 \\
1457 \\
3.389\end{array}$ & $\begin{array}{l}40.81 \\
1490 . \\
3.435\end{array}$ & $\begin{array}{l}41.48 \\
1523 . \\
3.482\end{array}$ & $\begin{array}{l}42.14 \\
1556 . \\
3.528\end{array}$ & $\begin{array}{l}42.80 \\
1589 . \\
3.574\end{array}$ & $\begin{array}{l}43.45 \\
1623 \\
3.620\end{array}$ & $\begin{array}{l}44.111 \\
1657 \\
3.665\end{array}$ & $\begin{array}{l}44.76 \\
1691 . \\
3.710\end{array}$ & $\begin{array}{l}45.41 \\
1726 . \\
3.755\end{array}$ \\
\hline 2500 & $\begin{array}{l}\nabla \\
8 \\
5\end{array}$ & & $\begin{array}{l}37.21 \\
1392 . \\
3.288\end{array}$ & $\begin{array}{l}37.86 \\
1424 . \\
3.335\end{array}$ & $\begin{array}{l}38.50 \\
1457 . \\
3.382\end{array}$ & $\begin{array}{l}39.14 \\
1489 . \\
3.429\end{array}$ & $\begin{array}{l}39.78 \\
1522 . \\
3.475\end{array}$ & $\begin{array}{l}40.42 \\
1555 . \\
3.522\end{array}$ & $\begin{array}{l}91.05 \\
1589 \\
3.567\end{array}$ & $\begin{array}{l}41.69 \\
1623 \\
3.613\end{array}$ & $\begin{array}{l}42.32 \\
1657 \\
3.658\end{array}$ & $\begin{array}{l}42.94 \\
1691 \\
3.704\end{array}$ & $\begin{array}{l}43.57 \\
1725 . \\
3.748\end{array}$ \\
\hline 2600 & $\begin{array}{l}y \\
y \\
5\end{array}$ & & $\begin{array}{l}35.73 \\
1392 . \\
3.281\end{array}$ & $\begin{array}{l}36.36 \\
1424 . \\
3.329\end{array}$ & $\begin{array}{l}36.98 \\
1456 . \\
3.376\end{array}$ & $\begin{array}{l}37.60 \\
1489 . \\
3.423\end{array}$ & $\begin{array}{l}38.22 \\
1522 . \\
3.469\end{array}$ & $\begin{array}{l}38.83 \\
1555 \\
3.515\end{array}$ & $\begin{array}{l}39.45 \\
1588 . \\
3.561\end{array}$ & $\begin{array}{l}40.06 \\
1622 \\
3.607\end{array}$ & $\begin{array}{l}40.66 \\
1656 . \\
3.652\end{array}$ & $\begin{array}{l}41.27 \\
1690 \\
3.697\end{array}$ & $\begin{array}{l}41.88 \\
1725 . \\
3.742\end{array}$ \\
\hline 2700 & $\begin{array}{l}\nabla \\
y \\
S\end{array}$ & & $\begin{array}{l}34.36 \\
1391 \\
3.275\end{array}$ & $\begin{array}{l}34.97 \\
1423 . \\
3.323\end{array}$ & $\begin{array}{l}35.57 \\
1455 . \\
3.370\end{array}$ & $\begin{array}{l}36.17 \\
1488 \\
3.417\end{array}$ & $\begin{array}{l}36.77 \\
1521 . \\
3.463\end{array}$ & $\begin{array}{l}37.36 \\
1550 \\
3.509\end{array}$ & $\begin{array}{l}37.96 \\
1588 . \\
3.555\end{array}$ & $\begin{array}{l}38.55 \\
1622 \\
3.601\end{array}$ & $\begin{array}{l}39.13 \\
1655 \\
3.646\end{array}$ & $\begin{array}{l}39.72 \\
1690 \\
3.691\end{array}$ & $\begin{array}{l}40.31 \\
1724 . \\
3.736\end{array}$ \\
\hline 2800 & $\begin{array}{l}y \\
y \\
S\end{array}$ & & $\begin{array}{l}33.10 \\
1390 . \\
3.269\end{array}$ & $\begin{array}{l}33.68 \\
1422 \\
3.317\end{array}$ & $\begin{array}{l}34.27 \\
1455 . \\
3.364\end{array}$ & $\begin{array}{l}34.85 \\
1488 . \\
3.411\end{array}$ & $\begin{array}{l}35.43 \\
1521 . \\
3.457\end{array}$ & $\begin{array}{l}36.00 \\
1554 \\
3.503\end{array}$ & $\begin{array}{l}36.57 \\
1587 \\
3.549\end{array}$ & $\begin{array}{l}37.15 \\
1621 \\
3.595\end{array}$ & $\begin{array}{l}37.72 \\
1655 . \\
3.641\end{array}$ & $\begin{array}{l}38.28 \\
1689 \\
3.68 E\end{array}$ & $\begin{array}{l}38.85 \\
1724 . \\
3.731\end{array}$ \\
\hline
\end{tabular}


Table 12 (Continued)

Temperature range: 400 to $500^{\circ} \mathrm{C}$

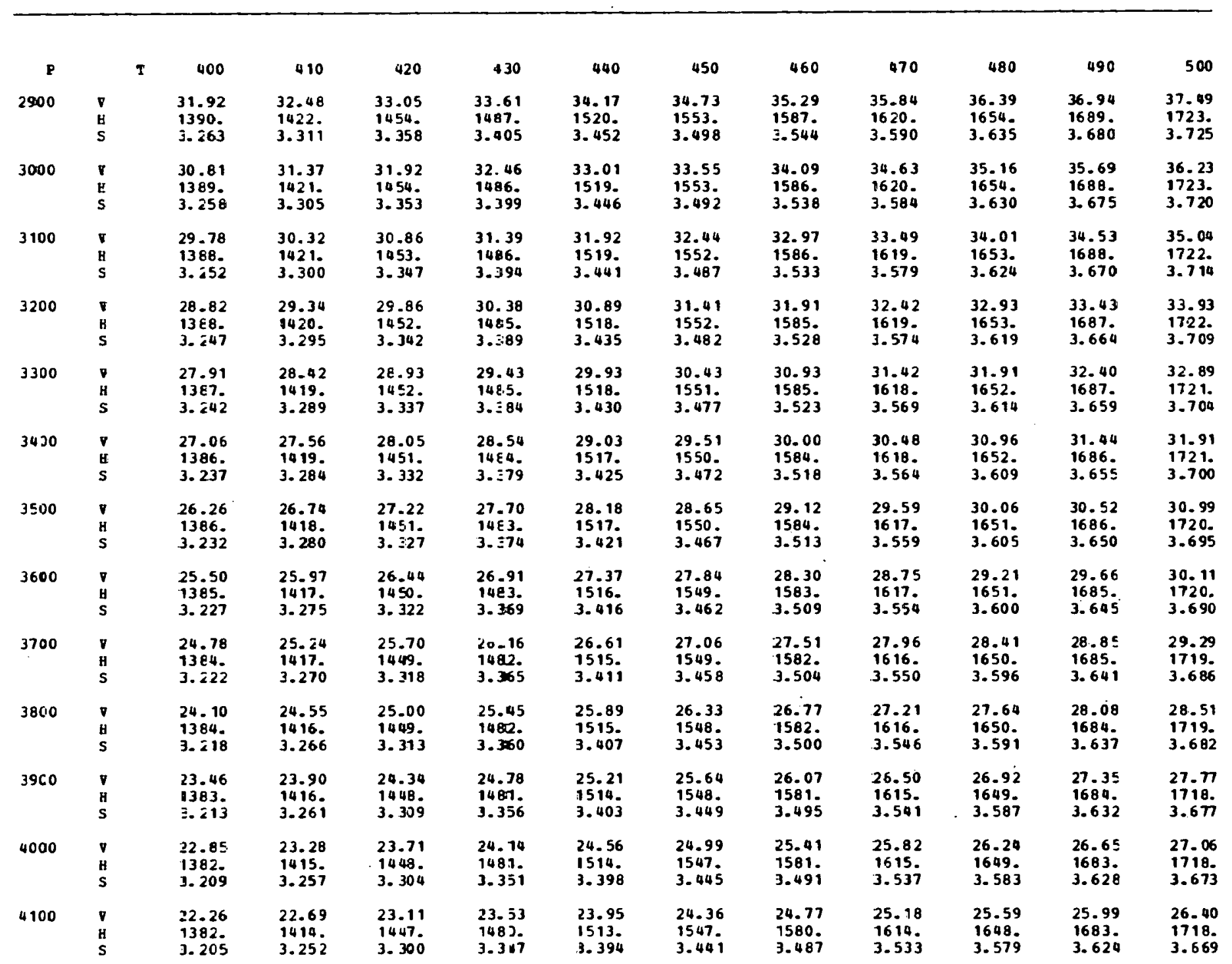


Table 12 (Continued)

Temperature range: 400 to $500^{\circ} \mathrm{C}$

\begin{tabular}{|c|c|c|c|c|c|c|c|c|c|c|c|c|}
\hline P & $I$ & 400 & 410 & 420 & 430 & 440 & 450 & 460 & 470 & 480 & 490 & 500 \\
\hline 4200 & $\begin{array}{l}\mathbf{V} \\
\mathbf{H} \\
\mathbf{S}\end{array}$ & $\begin{array}{l}21.71 \\
1381 . \\
3.200\end{array}$ & $\begin{array}{l}22.13 \\
1410 . \\
3.248\end{array}$ & $\begin{array}{l}22.54 \\
1446 . \\
3.296\end{array}$ & $\begin{array}{l}22.95 \\
1079 \\
3.343\end{array}$ & $\begin{array}{l}23.36 \\
1513 . \\
3.390\end{array}$ & $\begin{array}{l}23.77 \\
1546 . \\
3.437\end{array}$ & $\begin{array}{l}24.17 \\
1580 . \\
3.483\end{array}$ & $\begin{array}{l}24.57 \\
1614 . \\
3.529\end{array}$ & $\begin{array}{l}20.97 \\
1648 . \\
3.575\end{array}$ & $\begin{array}{l}25.36 \\
1682 . \\
3.620\end{array}$ & $\begin{array}{l}25.76 \\
1717 . \\
3.665\end{array}$ \\
\hline 4300 & $\begin{array}{l}\mathbf{1} \\
\mathrm{B} \\
\mathbf{S}\end{array}$ & $\begin{array}{l}21.18 \\
1381 \\
3.196\end{array}$ & $\begin{array}{l}21.59 \\
1413 . \\
3.244\end{array}$ & $\begin{array}{l}22.00 \\
1446 . \\
3.292\end{array}$ & $\begin{array}{l}22.40 \\
1479 . \\
3.339\end{array}$ & $\begin{array}{l}22.80 \\
1512 . \\
3.386\end{array}$ & $\begin{array}{l}23.20 \\
1546 . \\
3.933\end{array}$ & $\begin{array}{l}23.59 \\
1579 . \\
3.479\end{array}$ & $\begin{array}{l}23.99 \\
1613 . \\
3.525\end{array}$ & $\begin{array}{l}24.38 \\
1647 \\
3.571\end{array}$ & $\begin{array}{l}24.76 \\
1682 . \\
3.616\end{array}$ & $\begin{array}{l}25.15 \\
171.7 \\
3.661\end{array}$ \\
\hline 4400 & $\begin{array}{l}\mathbf{T} \\
\mathbf{H} \\
\mathbf{S}\end{array}$ & $\begin{array}{l}20.68 \\
1380 . \\
3.192\end{array}$ & $\begin{array}{l}21.08 \\
1412 \\
3.240\end{array}$ & $\begin{array}{l}21.48 \\
1445 . \\
3.288\end{array}$ & $\begin{array}{l}21.88 \\
1478 . \\
3.335\end{array}$ & $\begin{array}{l}22.27 \\
1511 . \\
3.382\end{array}$ & $\begin{array}{l}22.66 \\
1545 . \\
3.429\end{array}$ & $\begin{array}{l}23.04 \\
1579 . \\
3.475\end{array}$ & $\begin{array}{l}23.43 \\
1613 . \\
3.521\end{array}$ & $\begin{array}{l}23.81 \\
1647 \\
3.567\end{array}$ & $\begin{array}{l}24.19 \\
1681 . \\
3.612\end{array}$ & $\begin{array}{l}24.57 \\
1716 . \\
3.657\end{array}$ \\
\hline 4500 & $\begin{array}{l}7 \\
8 \\
5\end{array}$ & $\begin{array}{l}20.20 \\
1379 . \\
3.188\end{array}$ & $\begin{array}{l}20.59 \\
1012 . \\
3.236\end{array}$ & $\begin{array}{l}20.99 \\
1445 \\
3.284\end{array}$ & $\begin{array}{l}21.37 \\
1478 \\
3.331\end{array}$ & $\begin{array}{l}21.76 \\
1511 . \\
3.378\end{array}$ & $\begin{array}{l}22.14 \\
1544 . \\
3.425\end{array}$ & $\begin{array}{l}22.52 \\
1578 \\
3.479\end{array}$ & $\begin{array}{l}22.90 \\
1612 \\
3.517\end{array}$ & $\begin{array}{l}23.27 \\
1646 \\
3.563\end{array}$ & $\begin{array}{l}23.65 \\
1681 . \\
3.609\end{array}$ & $\begin{array}{l}24.02 \\
1716 . \\
3.654\end{array}$ \\
\hline 4600 & $\begin{array}{l}\mathbf{v} \\
\mathbf{u} \\
\mathbf{S}\end{array}$ & $\begin{array}{l}19.74 \\
1379 \\
3.184\end{array}$ & $\begin{array}{l}20.13 \\
1411 . \\
3.232\end{array}$ & $\begin{array}{l}20.51 \\
1444 \\
3.280\end{array}$ & $\begin{array}{l}20.89 \\
1477 \\
3.327\end{array}$ & $\begin{array}{l}21.27 \\
1510 . \\
3.374\end{array}$ & $\begin{array}{l}21.65 \\
1544 . \\
3.421\end{array}$ & $\begin{array}{l}22.02 \\
1578 \\
3.467\end{array}$ & $\begin{array}{l}22.39 \\
1612 . \\
3.514 .\end{array}$ & $\begin{array}{l}22.76 \\
1646 \\
3.559\end{array}$ & $\begin{array}{l}23.13 \\
1680 \\
3.605\end{array}$ & $\begin{array}{l}23.49 \\
1715 \\
3.650\end{array}$ \\
\hline 4700 & $\begin{array}{l}\mathbf{7} \\
\mathbf{1} \\
\mathbf{S}\end{array}$ & $\begin{array}{l}19.30 \\
1378 . \\
3.180\end{array}$ & $\begin{array}{l}19.68 \\
1411 . \\
3.228\end{array}$ & $\begin{array}{l}20.06 \\
1443 . \\
3.276\end{array}$ & $\begin{array}{l}20.43 \\
1476 . \\
3.324\end{array}$ & $\begin{array}{l}20.81 \\
1510 . \\
3.371\end{array}$ & $\begin{array}{l}21.17 \\
1543 . \\
3.417\end{array}$ & $\begin{array}{l}21.54 \\
1577 . \\
3.464\end{array}$ & $\begin{array}{l}21.90 \\
1611 \\
3.510\end{array}$ & $\begin{array}{l}22.27 \\
1645 \\
3.556\end{array}$ & $\begin{array}{l}22.63 \\
1680 \\
3.60 i\end{array}$ & $\begin{array}{l}22.98 \\
1715 \\
3.646\end{array}$ \\
\hline 4800 & $\begin{array}{l}\nabla \\
\mathbf{B} \\
\mathbf{S}\end{array}$ & $\begin{array}{l}18.88 \\
1377 \\
3.177\end{array}$ & $\begin{array}{l}19.26 \\
1410 . \\
3.225\end{array}$ & $\begin{array}{l}19.63 \\
1443 \\
3.272\end{array}$ & $\begin{array}{l}19.99 \\
1476 . \\
3.320\end{array}$ & $\begin{array}{l}20.36 \\
1509 \\
3.367\end{array}$ & $\begin{array}{l}20.72 \\
1543 . \\
3.414\end{array}$ & $\begin{array}{l}217.08 \\
1577 . \\
3.460\end{array}$ & $\begin{array}{l}21.04 \\
1611 . \\
3.506\end{array}$ & $\begin{array}{l}21.79 \\
1645 \\
3.552\end{array}$ & $\begin{array}{l}22.15 \\
1680 . \\
3.598\end{array}$ & $\begin{array}{l}22.50 \\
1714 . \\
3.643\end{array}$ \\
\hline 4900 & $\begin{array}{l}\mathbf{y} \\
\mathbf{y} \\
\mathbf{S}\end{array}$ & $\begin{array}{l}18.48 \\
1377 \\
3.173\end{array}$ & $\begin{array}{l}18.85 \\
1409 . \\
3.221\end{array}$ & $\begin{array}{l}19.21 \\
1442 . \\
3.269\end{array}$ & $\begin{array}{l}19.57 \\
1475 . \\
3.316\end{array}$ & $\begin{array}{l}19.93 \\
1509 . \\
3.363\end{array}$ & $\begin{array}{l}20.29 \\
1542 . \\
3.410\end{array}$ & $\begin{array}{l}20.64 \\
1576 . \\
3.457\end{array}$ & $\begin{array}{l}20.99 \\
1610 . \\
3.503\end{array}$ & $\begin{array}{l}21.34 \\
1644 \\
3.549\end{array}$ & $\begin{array}{l}21.69 \\
1679 . \\
3.594\end{array}$ & $\begin{array}{l}22.00 \\
1714 \\
3.639\end{array}$ \\
\hline 5000 & $\begin{array}{l}\mathbf{y} \\
\mathbf{y} \\
\mathbf{s}\end{array}$ & $\begin{array}{l}18.09 \\
1376 . \\
3.169\end{array}$ & $\begin{array}{l}18.45 \\
1409 \\
3.217\end{array}$ & $\begin{array}{l}18.81 \\
1442 . \\
3.265\end{array}$ & $\begin{array}{l}19.17 \\
1475 \\
3.313\end{array}$ & $\begin{array}{l}19.52 \\
1508 \\
3.360\end{array}$ & $\begin{array}{l}19.87 \\
1542 . \\
3.407\end{array}$ & $\begin{array}{l}20.22 \\
1576 . \\
3.453\end{array}$ & $\begin{array}{l}20.57 \\
1610 \\
3.499\end{array}$ & $\begin{array}{l}20.91 \\
1644 . \\
3.545\end{array}$ & $\begin{array}{l}21.25 \\
1679 . \\
3.591\end{array}$ & $\begin{array}{l}21.59 \\
1713 . \\
3.636\end{array}$ \\
\hline 5200 & $\begin{array}{l}y \\
\mathbf{u} \\
\mathbf{s}\end{array}$ & $\begin{array}{l}17.36 \\
1375 . \\
3.162\end{array}$ & $\begin{array}{l}17.7 \% \\
1407 . \\
3.210\end{array}$ & $\begin{array}{l}18.06 \\
1440 . \\
3.258\end{array}$ & $\begin{array}{l}18.41 \\
1474 . \\
3.306\end{array}$ & $\begin{array}{l}18.75 \\
1507 . \\
3.353\end{array}$ & $\begin{array}{l}19.09 \\
1541 . \\
3.400\end{array}$ & $\begin{array}{l}19.42 \\
1575 . \\
3.446\end{array}$ & $\begin{array}{l}19.76 \\
1609 . \\
3.492\end{array}$ & $\begin{array}{l}20.09 \\
1643 . \\
3.538\end{array}$ & $\begin{array}{l}20 \vdots 42 \\
1678 \\
3.58 \text { i }\end{array}$ & $\begin{array}{l}20.75 \\
1712 . \\
3.629\end{array}$ \\
\hline 5400 & $\begin{array}{l}\mathbf{7} \\
\mathbf{B} \\
\mathbf{S}\end{array}$ & $\begin{array}{l}16.69 \\
1373 . \\
3.155\end{array}$ & $\begin{array}{l}17.03 \\
1406 \\
3.203\end{array}$ & $\begin{array}{l}17.37 \\
1439 \\
3.251\end{array}$ & $\begin{array}{l}17.70 \\
1472 . \\
3.299\end{array}$ & $\begin{array}{l}18.03 \\
1506 . \\
3.346\end{array}$ & $\begin{array}{l}18.36 \\
1540 . \\
3.393\end{array}$ & $\begin{array}{l}18.69 \\
1579 . \\
3.440\end{array}$ & $\begin{array}{l}19.01 \\
1608 \\
3.486\end{array}$ & $\begin{array}{l}19.34 \\
1642 \\
3.532\end{array}$ & $\begin{array}{l}19.66 \\
1677 . \\
3.578\end{array}$ & $\begin{array}{l}19.97 \\
1712 . \\
3.623\end{array}$ \\
\hline 5600 & $\begin{array}{l}\mathbf{Y} \\
\mathbf{H} \\
\mathbf{S}\end{array}$ & $\begin{array}{l}16.06 \\
1372 . \\
3.148\end{array}$ & $\begin{array}{l}16.40 \\
1405 \\
3.197\end{array}$ & $\begin{array}{l}16.72 \\
1438 . \\
3.245\end{array}$ & $\begin{array}{l}17.05 \\
1471 . \\
3.292\end{array}$ & $\begin{array}{l}17.37 \\
1505 . \\
3.390\end{array}$ & $\begin{array}{l}17.69 \\
1539 \\
3.387\end{array}$ & $\begin{array}{l}18.01 \\
1572 . \\
3.433\end{array}$ & $\begin{array}{l}18.32 \\
1607 . \\
3.480\end{array}$ & $\begin{array}{l}18.63 \\
1641 . \\
3.526\end{array}$ & $\begin{array}{l}18.95 \\
1676 \\
3.57 i\end{array}$ & $\begin{array}{l}19: 25 \\
1711 . \\
3.671\end{array}$ \\
\hline 5800 & $\begin{array}{l}\mathbf{v} \\
\text { B } \\
\mathbf{S}\end{array}$ & $\begin{array}{l}15.48 \\
1371 . \\
3.142\end{array}$ & $\begin{array}{l}15.81 \\
1404 . \\
3.190\end{array}$ & $\begin{array}{l}16.13 \\
1437 . \\
3.238\end{array}$ & $\begin{array}{l}16.44 \\
1470 . \\
3.286\end{array}$ & $\begin{array}{l}16.76 \\
1504 \\
3.333\end{array}$ & $\begin{array}{l}17.07 \\
1537 \\
3.380\end{array}$ & $\begin{array}{l}17.37 \\
1571 \\
3.427\end{array}$ & $\begin{array}{l}17.68 \\
1606 \\
3.473\end{array}$ & $\begin{array}{l}17.98 \\
1640_{-} \\
3.519\end{array}$ & $\begin{array}{l}18.28 \\
1675 \\
3.565\end{array}$ & $\begin{array}{l}18.58 \\
17110 . \\
3.611\end{array}$ \\
\hline
\end{tabular}


Táble 12 (Continued)

Tempercture range: 400 to $500^{\circ} \mathrm{C}$

\begin{tabular}{|c|c|c|c|c|c|c|c|c|c|c|c|c|c|}
\hline p & & I & 400 & +10 & 420 & 430 & 440 & 450 & 460 & 470 & 480 & 490 & 500 \\
\hline$\epsilon 000$ & $\begin{array}{l}\mathbf{y} \\
\mathbf{g} \\
\mathbf{s}\end{array}$ & & $\begin{array}{l}14.94 \\
1370 . \\
3.135\end{array}$ & $\begin{array}{l}15.26 \\
1403 . \\
3.184\end{array}$ & $\begin{array}{l}15.57 \\
1436 . \\
3.232\end{array}$ & $\begin{array}{l}15.88 \\
1769 . \\
3.280\end{array}$ & $\begin{array}{l}16.18 \\
1503 . \\
3.327\end{array}$ & $\begin{array}{l}16.48 \\
1536 \\
3.374\end{array}$ & $\begin{array}{l}16.78 \\
1570 . \\
3.421\end{array}$ & $\begin{array}{l}17.08 \\
1605 \\
3.067\end{array}$ & $\begin{array}{l}17.38 \\
1639 . \\
3.513\end{array}$ & $\begin{array}{l}17.67 \\
1674 . \\
3.559\end{array}$ & $\begin{array}{l}17.96 \\
1709 . \\
3.605\end{array}$ \\
\hline 6200 & $\begin{array}{l}\nabla \\
\mathbf{u} \\
\mathrm{S}\end{array}$ & & $\begin{array}{l}14.44 \\
1368 . \\
3.129\end{array}$ & $\begin{array}{l}14.75 \\
1401 . \\
3.178\end{array}$ & $\begin{array}{l}15.05 \\
1435 . \\
3.226\end{array}$ & $\begin{array}{l}15.35 \\
1768 \\
3.274\end{array}$ & $\begin{array}{l}15.65 \\
1502 . \\
3.321\end{array}$ & $\begin{array}{l}15.94 \\
1535 . \\
3.368\end{array}$ & $\begin{array}{l}16.23 \\
1569 . \\
3.415\end{array}$ & $\begin{array}{l}16.52 \\
1604 . \\
3.462\end{array}$ & $\begin{array}{l}16.81 \\
1638 . \\
3.508\end{array}$ & $\begin{array}{l}17.09 \\
1673 . \\
3.553\end{array}$ & $\begin{array}{l}17.38 \\
1708 . \\
3.599\end{array}$ \\
\hline 6400 & $\begin{array}{l}V \\
\mathrm{H} \\
\mathrm{S}\end{array}$ & & $\begin{array}{l}13.97 \\
1367 \\
3.123\end{array}$ & $\begin{array}{l}14.27 \\
1400 \\
3.172\end{array}$ & $\begin{array}{l}14.56 \\
9433 \\
3.220\end{array}$ & $\begin{array}{l}14.85 \\
11667 \\
3.268\end{array}$ & $\begin{array}{l}15.14 \\
1500 . \\
3.315\end{array}$ & $\begin{array}{l}15.43 \\
1534 . \\
3.362\end{array}$ & $\begin{array}{l}15.71 \\
1568 . \\
3.409\end{array}$ & $\begin{array}{l}16.00 \\
1603 \\
3.456\end{array}$ & $\begin{array}{l}16.28 \\
1637 \\
3.502\end{array}$ & $\begin{array}{l}16.55 \\
1672 . \\
3.548\end{array}$ & $\begin{array}{l}16.83 \\
1707 . \\
3.593\end{array}$ \\
\hline 6600 & $\begin{array}{l}P \\
\text { H } \\
S\end{array}$ & & $\begin{array}{l}13.52 \\
1366 . \\
3.117\end{array}$ & $\begin{array}{l}33.82 \\
13.99 . \\
3.166\end{array}$ & $\begin{array}{l}94.10 \\
9432 . \\
3.214\end{array}$ & $\begin{array}{l}18.39 \\
1466 \\
3.262\end{array}$ & $\begin{array}{l}14.67 \\
1499 . \\
3.310\end{array}$ & $\begin{array}{l}14.95 \\
1533 \\
3.357\end{array}$ & $\begin{array}{l}15.23 \\
1567 \\
3.404\end{array}$ & $\begin{array}{l}15.51 \\
1602 . \\
3.450\end{array}$ & $\begin{array}{l}15.78 \\
1636 . \\
3.496\end{array}$ & $\begin{array}{l}16.05 \\
1671 . \\
3.542\end{array}$ & $\begin{array}{l}16.32 \\
1706 . \\
3.588\end{array}$ \\
\hline 6800 & $\begin{array}{l}\nabla \\
\mathrm{g} \\
\mathrm{s}\end{array}$ & & $\begin{array}{l}13.11 \\
1365 \\
3.111\end{array}$ & $\begin{array}{l}13.39 \\
1398 . \\
3.160\end{array}$ & $\begin{array}{l}13.68 \\
1431 . \\
3.208\end{array}$ & $\begin{array}{l}13.95 \\
1165 . \\
3.256\end{array}$ & $\begin{array}{l}14.23 \\
1498 . \\
3.304\end{array}$ & $\begin{array}{l}14.50 \\
1532 \\
3.351\end{array}$ & $\begin{array}{l}14.77 \\
1566 . \\
3.398\end{array}$ & $\begin{array}{l}15.04 \\
1601 . \\
3.445\end{array}$ & $\begin{array}{l}15.31 \\
1635 \\
3.491\end{array}$ & $\begin{array}{l}15.57 \\
1670 . \\
3.537\end{array}$ & $\begin{array}{l}15.84 \\
1705 . \\
3.583\end{array}$ \\
\hline 7000 & $\begin{array}{l}7 \\
\text { H } \\
\text { S }\end{array}$ & & $\begin{array}{l}12.72 \\
13 \in 3 . \\
3.106\end{array}$ & $\begin{array}{l}13.00 \\
1397 \\
3.156\end{array}$ & $\begin{array}{l}13.27 \\
1130 . \\
3.203\end{array}$ & $\begin{array}{l}13.55 \\
1763 . \\
3.251\end{array}$ & $\begin{array}{l}13.81 \\
1497 \\
3.299\end{array}$ & $\begin{array}{l}14.08 \\
1531 . \\
3.346\end{array}$ & $\begin{array}{l}14.35 \\
1565 \\
3.393\end{array}$ & $\begin{array}{l}14.61 \\
1600 \\
3.440\end{array}$ & $\begin{array}{l}14.87 \\
1634 . \\
3.486\end{array}$ & $\begin{array}{l}15.13 \\
1669 . \\
3.532\end{array}$ & $\begin{array}{l}15.38 \\
1704 . \\
3.578\end{array}$ \\
\hline 7500 & $\begin{array}{l}P \\
H \\
S\end{array}$ & & $\begin{array}{l}11.83 \\
1360 . \\
3.092\end{array}$ & $\begin{array}{l}12.10 \\
13: 4 . \\
3.41\end{array}$ & $\begin{array}{l}12.36 \\
1427 \\
3.190\end{array}$ & $\begin{array}{l}12.62 \\
1461 . \\
3.238\end{array}$ & $\begin{array}{l}12.87 \\
1495 \\
3.286\end{array}$ & $\begin{array}{l}13.13 \\
1529 \\
3.333\end{array}$ & $\begin{array}{l}13.38 \\
1563 \\
3.380\end{array}$ & $\begin{array}{l}13.63 \\
1597 \\
3.427\end{array}$ & $\begin{array}{l}13.87 \\
1632 . \\
3.073\end{array}$ & $\begin{array}{l}14.12 \\
1667 . \\
3.519\end{array}$ & $\begin{array}{l}14.36 \\
1702 . \\
3.565\end{array}$ \\
\hline 8000 & $\begin{array}{l}P \\
y \\
s\end{array}$ & & $\begin{array}{l}11.07 \\
1357 \\
3.079\end{array}$ & $\begin{array}{l}11.32 \\
1391 . \\
3.28\end{array}$ & $\begin{array}{l}11.57 \\
1424 \\
3.177\end{array}$ & $\begin{array}{l}11.81 \\
1458 . \\
3.225\end{array}$ & $\begin{array}{l}12.06 \\
1492 \\
3.273\end{array}$ & $\begin{array}{l}12.30 \\
1526 \\
3.321\end{array}$ & $\begin{array}{l}12.53 \\
1561 . \\
3.368\end{array}$ & $\begin{array}{l}12.77 \\
1595 \\
3.415\end{array}$ & $\begin{array}{l}13.00 \\
1630 \\
3.461\end{array}$ & $\begin{array}{l}13.23 \\
1665 \\
3.508\end{array}$ & $\begin{array}{l}13.46 \\
1700 . \\
3.553\end{array}$ \\
\hline 8500 & $\begin{array}{l}\nabla \\
\mathbf{y} \\
\mathrm{S}\end{array}$ & & $\begin{array}{l}10.39 \\
1354 . \\
3.067\end{array}$ & $\begin{array}{l}10.64 \\
138_{0} \\
3 .: 16\end{array}$ & $\begin{array}{l}10.87 \\
1422 . \\
3.165\end{array}$ & $\begin{array}{l}11.11 \\
1455 . \\
3.213\end{array}$ & $\begin{array}{l}11.34 \\
1489 . \\
3.261\end{array}$ & $\begin{array}{l}11.57 \\
1524 . \\
3.309\end{array}$ & $\begin{array}{l}11.79 \\
1558 . \\
3.356\end{array}$ & $\begin{array}{l}12.02 \\
1593 . \\
3.403\end{array}$ & $\begin{array}{l}12.24 \\
1628 \\
3.450\end{array}$ & $\begin{array}{l}12.46 \\
1663 \\
3.496\end{array}$ & $\begin{array}{l}12.68 \\
1698 . \\
3.542\end{array}$ \\
\hline 9000 & $\begin{array}{l}y \\
y \\
5\end{array}$ & & $\begin{array}{l}9.801 \\
1351 . \\
3.055\end{array}$ & $\begin{array}{l}10.03 \\
138.5 \\
3.504\end{array}$ & $\begin{array}{l}10.26 \\
1419 . \\
3.153\end{array}$ & $\begin{array}{l}10.48 \\
1453 . \\
3.202\end{array}$ & $\begin{array}{l}10.70 \\
1487 \\
3.250\end{array}$ & $\begin{array}{l}10.92 \\
1521 \\
3.298\end{array}$ & $\begin{array}{l}11.10 \\
1556 \\
3.345\end{array}$ & $\begin{array}{l}11: 35 \\
1591 \\
3.393\end{array}$ & $\begin{array}{l}11.57 \\
1625 \\
3.439\end{array}$ & $\begin{array}{l}11.78 \\
1661 . \\
3.486\end{array}$ & $\begin{array}{l}11.98 \\
1696 . \\
3.532\end{array}$ \\
\hline 10000 & $\begin{array}{l}7 \\
y \\
5\end{array}$ & & $\begin{array}{l}8.803 \\
1346 . \\
3.032\end{array}$ & $\begin{array}{l}9.615 \\
13 E 0 . \\
3.682\end{array}$ & $\begin{array}{l}9.224 \\
1414 \\
3.132\end{array}$ & $\begin{array}{l}9.430 \\
14.48 \\
3.181\end{array}$ & $\begin{array}{l}9.634 \\
1482 . \\
3.229\end{array}$ & $\begin{array}{l}9.835 \\
1517 \\
3.277\end{array}$ & $\begin{array}{l}10.03 \\
1551 . \\
3.325\end{array}$ & $\begin{array}{l}10.23 \\
1586 . \\
3.372\end{array}$ & $\begin{array}{l}10.43 \\
1621 . \\
3.419\end{array}$ & $\begin{array}{l}10.62 \\
1657 \\
3.466\end{array}$ & $\begin{array}{l}10.81 \\
1692 . \\
3.512\end{array}$ \\
\hline 11500 & $\begin{array}{l}\mathbf{V} \\
H \\
\mathbf{S}\end{array}$ & & $\begin{array}{l}8.000 \\
1340 . \\
3.012\end{array}$ & $\begin{array}{l}8.96 \\
1374 . \\
3 . C 62\end{array}$ & $\begin{array}{l}\text { 8. } 390 \\
1408 . \\
3.112\end{array}$ & $\begin{array}{l}8.581 \\
1643 . \\
3.161\end{array}$ & $\begin{array}{l}8.770 \\
1477 . \\
3.210\end{array}$ & $\begin{array}{l}8.956 \\
1512 . \\
3.258\end{array}$ & $\begin{array}{l}9.141 \\
1547 . \\
3.306\end{array}$ & $\begin{array}{l}9.323 \\
1582 . \\
3.353\end{array}$ & $\begin{array}{l}9.503 \\
1617 \\
3.400\end{array}$ & $\begin{array}{l}9.682 \\
1653 \\
3.447\end{array}$ & $\begin{array}{l}9.858 \\
1688 . \\
3.493\end{array}$ \\
\hline 12.000 & $\begin{array}{l}T \\
H \\
S\end{array}$ & & $\begin{array}{l}7.342 \\
1335 . \\
2.993\end{array}$ & $\begin{array}{l}7.526 \\
13 \in 9 . \\
3.043\end{array}$ & $\begin{array}{l}7.706 \\
8404 \\
3.093\end{array}$ & $\begin{array}{l}7.884 \\
1438 \\
3.143\end{array}$ & $\begin{array}{l}8.060 \\
1473 . \\
3.192\end{array}$ & $\begin{array}{l}8.234 \\
1508 \\
3.240\end{array}$ & $\begin{array}{l}8.405 \\
1543 . \\
3.288\end{array}$ & $\begin{array}{l}8.575 \\
1578 \\
3.336\end{array}$ & $\begin{array}{l}8.743 \\
1613 . \\
3.383\end{array}$ & $\begin{array}{l}8.909 \\
1649 . \\
3.430\end{array}$ & $\begin{array}{l}9.073 \\
1689 . \\
3.476\end{array}$ \\
\hline
\end{tabular}


Table 12 (Continued)

Temperature range: 400 to $500^{\circ} \mathrm{C}$

\begin{tabular}{|c|c|c|c|c|c|c|c|c|c|c|c|c|c|}
\hline P & & $\mathbf{T}$ & 000 & 410 & 420 & 430 & 440 & 450 & 460 & 470 & 480 & 490 & 500 \\
\hline 13000 & $\begin{array}{l}7 \\
4 \\
5\end{array}$ & & $\begin{array}{l}6.797 \\
1330 . \\
2.975\end{array}$ & $\begin{array}{l}6.968 \\
1364 . \\
3.026\end{array}$ & $\begin{array}{l}7.137 \\
1399 . \\
3.076\end{array}$ & $\begin{array}{l}7.303 \\
1434 . \\
3.125\end{array}$ & $\begin{array}{l}7.468 \\
1469 . \\
3.175\end{array}$ & $\begin{array}{l}7.630 \\
1504 . \\
3.223\end{array}$ & $\begin{array}{l}7.791 \\
1539 . \\
3.272\end{array}$ & $\begin{array}{l}7.949 \\
1574 . \\
3.320\end{array}$ & $\begin{array}{l}8.106 \\
1609 \\
3.367\end{array}$ & $\begin{array}{l}8.262 \\
1645 . \\
3.414\end{array}$ & $\begin{array}{l}8.416 \\
1681 . \\
3.461\end{array}$ \\
\hline 14000 & $\begin{array}{l}\nabla \\
\text { s } \\
\text { s }\end{array}$ & & $\begin{array}{l}6.338 \\
1325 \\
2.958\end{array}$ & $\begin{array}{l}6.499 \\
1360 . \\
3.009\end{array}$ & $\begin{array}{l}6.657 \\
1395 . \\
3.060\end{array}$ & $\begin{array}{l}6.813 \\
1429 . \\
3.109\end{array}$ & $\begin{array}{l}6.968 \\
1464= \\
3.159\end{array}$ & $\begin{array}{l}7.120 \\
1500 \\
3.208\end{array}$ & $\begin{array}{l}7.271 \\
1535 . \\
3.256\end{array}$ & $\begin{array}{l}7.420 \\
1570 . \\
3.304\end{array}$ & $\begin{array}{l}7.567 \\
1606 . \\
3.352\end{array}$ & $\begin{array}{l}7.713 \\
1642 . \\
3.399\end{array}$ & $\begin{array}{l}7.858 \\
1678 . \\
3.446\end{array}$ \\
\hline $1 \leq 000$ & $\begin{array}{l}y \\
y \\
5\end{array}$ & & $\begin{array}{l}5.949 \\
1321 . \\
2.943\end{array}$ & $\begin{array}{l}6.100 \\
1356 \\
2.994\end{array}$ & $\begin{array}{l}6.248 \\
1391 . \\
3.004\end{array}$ & $\begin{array}{l}6.395 \\
1425 . \\
3.094\end{array}$ & $\begin{array}{l}6.541 \\
1461 \\
3.144\end{array}$ & $\begin{array}{l}6.684 \\
1196 . \\
3.193\end{array}$ & $\begin{array}{l}\epsilon .826 \\
1531 \\
3.242\end{array}$ & $\begin{array}{l}6.967 \\
1567 . \\
3.290\end{array}$ & $\begin{array}{l}7.106 \\
1603 . \\
3.338\end{array}$ & $\begin{array}{l}7.293 \\
1638 \\
3.38 \leq\end{array}$ & $\begin{array}{l}7.379 \\
1674 . \\
3.432\end{array}$ \\
\hline $1 \in 000$ & $\begin{array}{l}\nabla \\
\mathbf{D} \\
\mathbf{S}\end{array}$ & & $\begin{array}{l}5.615 \\
1317 . \\
2.928\end{array}$ & $\begin{array}{l}5.757 \\
1352 . \\
2.979\end{array}$ & $\begin{array}{l}5.897 \\
1387 . \\
3.030\end{array}$ & $\begin{array}{l}6.036 \\
1422 . \\
3.080\end{array}$ & $\begin{array}{l}6.173 \\
1457 . \\
3.130\end{array}$ & $\begin{array}{l}6.308 \\
1492 \\
3.179\end{array}$ & $\begin{array}{l}6.442 \\
1528 \\
3.228\end{array}$ & $\begin{array}{l}6.575 \\
1563 \\
3.276\end{array}$ & $\begin{array}{l}6.706 \\
1599 . \\
3.324\end{array}$ & $\begin{array}{l}6.836 \\
1635 \\
3.37 \bar{i}\end{array}$ & $\begin{array}{l}6.965 \\
1671 . \\
3.419\end{array}$ \\
\hline 17000 & $\begin{array}{l}D \\
8 \\
S\end{array}$ & & $\begin{array}{l}5.326 \\
1313 . \\
2.914\end{array}$ & $\begin{array}{l}5.460 \\
1348 \\
2.966\end{array}$ & $\begin{array}{l}5.592 \\
1383 . \\
3.016\end{array}$ & $\begin{array}{l}5.723 \\
1418 \\
3.067\end{array}$ & $\begin{array}{l}5.853 \\
1453 \\
3.117\end{array}$ & $\begin{array}{l}5.981 \\
1489 . \\
3.166\end{array}$ & $\begin{array}{l}6.108 \\
1525 \\
3.215\end{array}$ & $\begin{array}{l}6.234 \\
1560 . \\
3.263\end{array}$ & $\begin{array}{l}6.358 \\
1596 \\
3.311\end{array}$ & $\begin{array}{l}6.481 \\
1632 \\
3.359\end{array}$ & $\begin{array}{l}6.604 \\
1669 . \\
3.406\end{array}$ \\
\hline 18000 & $\begin{array}{l}7 \\
8 \\
5\end{array}$ & & $\begin{array}{l}5.075 \\
1309 \\
2.90 i\end{array}$ & $\begin{array}{l}5.201 \\
1344 . \\
2.953\end{array}$ & $\begin{array}{l}5.326 \\
1380 . \\
3.004\end{array}$ & $\begin{array}{l}5.450 \\
1415 . \\
3.054\end{array}$ & $\begin{array}{l}5.573 \\
1450 . \\
3.104\end{array}$ & $\begin{array}{l}5.695 \\
1486 . \\
3.154\end{array}$ & $\begin{array}{l}5.815 \\
1521 . \\
\vdots .203\end{array}$ & $\begin{array}{l}5.934 \\
1557 \\
3.25 i\end{array}$ & $\begin{array}{l}6.052 \\
1593 . \\
3.299\end{array}$ & $\begin{array}{l}6.169 \\
1630 \\
3.347\end{array}$ & $\begin{array}{l}6.286 \\
1666 . \\
3.394\end{array}$ \\
\hline 19000 & $\begin{array}{l}Y \\
B \\
S\end{array}$ & & $\begin{array}{l}4.854 \\
1306 . \\
2.889\end{array}$ & $\begin{array}{l}4.974 \\
1341 . \\
2.940\end{array}$ & $\begin{array}{l}5.092 \\
1376 \\
2.992\end{array}$ & $\begin{array}{l}5.210 \\
1412 \\
3.042\end{array}$ & $\begin{array}{l}5.326 \\
1447 . \\
3.092\end{array}$ & $\begin{array}{l}5.442 \\
1483 . \\
3.142\end{array}$ & $\begin{array}{l}5.556 \\
1519: \\
3.191\end{array}$ & $\begin{array}{l}5.670 \\
1555 . \\
3.240\end{array}$ & $\begin{array}{l}5.782 \\
1591 . \\
3.288\end{array}$ & $\begin{array}{l}5.893 \\
1627 \\
3.336\end{array}$ & $\begin{array}{l}6.004 \\
1663 \\
3.383\end{array}$ \\
\hline 20000 & $\begin{array}{l}8 \\
8 \\
5\end{array}$ & & $\begin{array}{l}0.659 \\
1303 . \\
2.877\end{array}$ & $\begin{array}{l}4.773 \\
1338 . \\
2.929\end{array}$ & $\begin{array}{l}4.885 \\
1373 . \\
2.980\end{array}$ & $\begin{array}{l}4.997 \\
1409 \\
3.03 i\end{array}$ & $\begin{array}{l}5.108 \\
1444 \\
3.081\end{array}$ & $\begin{array}{l}5.217 \\
1480 . \\
3.131\end{array}$ & $\begin{array}{l}5.326 \\
1516 \\
3.180\end{array}$ & $\begin{array}{l}5.434 \\
1552 \\
3.229\end{array}$ & $\begin{array}{l}5.54 i \\
1588 . \\
3.277\end{array}$ & $\begin{array}{l}5.648 \\
1624 \\
3.325\end{array}$ & $\begin{array}{l}5.753 \\
1661 . \\
3.372\end{array}$ \\
\hline 21000 & $\begin{array}{l}\mathbf{v} \\
\mathrm{a} \\
\mathrm{s}\end{array}$ & & $\begin{array}{l}9.486 \\
1300 . \\
2.866\end{array}$ & $\begin{array}{l}9.594 \\
1335 \\
2.91 \varepsilon\end{array}$ & $\begin{array}{l}4.701 \\
1371 . \\
2.969\end{array}$ & $\begin{array}{l}4.807 \\
1406 \\
3.020\end{array}$ & $\begin{array}{l}9.912 \\
1.42 \\
3.070\end{array}$ & $\begin{array}{l}5.017 \\
1477 \\
3.120\end{array}$ & $\begin{array}{l}5.121 \\
1513 . \\
3.169\end{array}$ & $\begin{array}{l}5.224 \\
1549 . \\
3.218\end{array}$ & $\begin{array}{l}5.326 \\
1586 . \\
3.267\end{array}$ & $\begin{array}{l}5.428 \\
1622 . \\
3.315\end{array}$ & $\begin{array}{l}5.528 \\
1659 . \\
3.362\end{array}$ \\
\hline 22000 & $\begin{array}{l}\mathbf{Y} \\
\mathrm{B} \\
\mathrm{S}\end{array}$ & & $\begin{array}{l}4.332 \\
1297 . \\
2.855\end{array}$ & $\begin{array}{l}4.434 \\
1333 \\
2.90 ?\end{array}$ & $\begin{array}{l}4.536 \\
1368 \\
2.959\end{array}$ & $\begin{array}{l}4.637 \\
1404 . \\
3.010\end{array}$ & $\begin{array}{l}4.738 \\
1439 \\
3.060\end{array}$ & $\begin{array}{l}4.837 \\
1475 \\
3.110\end{array}$ & $\begin{array}{l}4.937 \\
1511 \\
3.159\end{array}$ & $\begin{array}{l}5.035 \\
1547 . \\
3.208\end{array}$ & $\begin{array}{l}5.133 \\
1583 . \\
3.257\end{array}$ & $\begin{array}{l}5.230 \\
16.20 . \\
3.305\end{array}$ & $\begin{array}{l}5.326 \\
1656 \\
3.352\end{array}$ \\
\hline 23000 & $\begin{array}{l}V \\
\text { D } \\
\text { S }\end{array}$ & & $\begin{array}{l}0.193 \\
1295 \\
2845\end{array}$ & $\begin{array}{l}4.297 \\
1330 \\
2.897\end{array}$ & $\begin{array}{l}4.388 \\
1366 \\
2.9^{\prime 4} 9\end{array}$ & $\begin{array}{l}4.484 \\
1401 \\
3.000\end{array}$ & $\begin{array}{l}4.580 \\
1437 \\
3.050\end{array}$ & $\begin{array}{l}4.676 \\
1473 . \\
3.100\end{array}$ & $\begin{array}{l}4.770 \\
1509 . \\
3.150\end{array}$ & $\begin{array}{l}4.864 \\
1545 . \\
3.199 .\end{array}$ & $\begin{array}{l}0.958 \\
1581 . \\
3.247\end{array}$ & $\begin{array}{l}5.051 \\
1618 . \\
3.295\end{array}$ & $\begin{array}{l}5.143 \\
1654 . \\
3.343\end{array}$ \\
\hline 24000 & $\begin{array}{l}V \\
\mathbf{H} \\
\mathrm{S}\end{array}$ & & $\begin{array}{l}4.068 \\
1293 . \\
2.036\end{array}$ & $\begin{array}{l}4.161 \\
1328 . \\
2.888\end{array}$ & $\begin{array}{l}4.254 \\
1363 . \\
2.939\end{array}$ & $\begin{array}{l}4.346 \\
1399 . \\
2.990\end{array}$ & $\begin{array}{l}4.438 \\
1435 \\
3.041\end{array}$ & $\begin{array}{l}4.529 \\
1471 . \\
3.091\end{array}$ & $\begin{array}{l}4.620 \\
1507 \\
3.140\end{array}$ & $\begin{array}{l}4.710 \\
1543 . \\
3.189\end{array}$ & $\begin{array}{l}4.800 \\
1579 . \\
3.238\end{array}$ & $\begin{array}{l}\text { 9. } 889 \\
1616 . \\
3.286\end{array}$ & $\begin{array}{l}4.977 \\
1652 . \\
3.330\end{array}$ \\
\hline 25000 & $\begin{array}{l}V \\
\mathrm{~B} \\
\mathrm{~S}\end{array}$ & & $\begin{array}{l}3.954 \\
1290 . \\
2.827\end{array}$ & $\begin{array}{l}4.043 \\
1326 \\
2.879\end{array}$ & $\begin{array}{l}4.132 \\
1361 . \\
2.930\end{array}$ & $\begin{array}{l}4.220 \\
1397 . \\
2.981\end{array}$ & $\begin{array}{l}4.308 \\
1433 \\
3.032\end{array}$ & $\begin{array}{l}4.396 \\
1469 . \\
3.082\end{array}$ & $\begin{array}{l}4.483 \\
1505 \\
3.13 i\end{array}$ & $\begin{array}{l}4.569 \\
1541 \\
3.181\end{array}$ & $\begin{array}{l}0.655 \\
1577 \\
3.229\end{array}$ & $\begin{array}{l}4.741 \\
1634 \\
3.277\end{array}$ & $\begin{array}{l}4.826 \\
1651 \\
3.325\end{array}$ \\
\hline
\end{tabular}




\section{REFERENCES}

1. J. J. Martin, "Equations of State," Ind. Eng. Chem., 59(12), 34--52 (1967).

2. S. L. Milora and J. W. Tester, Geothermal Energy as a Source of Electric Power, MIT Press, Cambridge, Massachusetts, 1976.

3. S. L. Milora, Application of the Martin Equation of State to the Thermodynamic Properties of Ammonia, ORNL/TM-4313 (December 1973).

4. S. L. Milora, STATEQ: A Nonlinear Least-Squares Code for Obtaining Martin Thermodynamic Representations of Fllids in the Gasenus and Dense Gaseous Regions, ORNL/TM-5115 (February 1976).

5. P. Davies, 'Thermodynamic Functions of Gases, Vo1. 1, edited by F. Din, Butterworths Scientific Publications, London, 1956.

6. J. A. Beattie and C. K. Lawrence, "Some of the Thermodynamic Properties of Ammonia - 1. The Compressibility of and an Equation of State for Gaseous Ammonia. The Vapor Pressure of Liquid Ammonia," J. Am. Chem. Soc., 52, 6-14 (1930).

7. "Tables of Thermodynamic Properties of Ammonia," Circular of the Bureau of Standards, No. 143: 1-14 (1923).

8. L. N. Canjar and F. S. Manning, Thermodynamic Properties and Reduced Correlations for Gases, Gulf Publishing Company, Houston, Texas, 1967 . 


\section{INTERNAL DISTRIBUTION}

$\begin{aligned} 1 . & \text { S. Barton } \\ 2 . & \text { S. E. Beal1 } \\ 3-12 . & \text { S. K. Combs } \\ 13 . & \text { W. E. Cooper } \\ 14 . & \text { W. B. Cottre11 } \\ 15 . & \text { F. L. Culler } \\ 16 . & \text { G. G. Fee } \\ 17 . & \text { W. Fulkerson } \\ 18 . & \text { M. J. Goglia } \\ 19 . & \text { J. F. Harvey } \\ 20 . & \text { H. W. Hoffman } \\ 21 . & \text { L. Levenson } \\ 22 . & \text { R. N. Lyon } \\ 23 . & \text { R. E. MacPherson } \\ 24 . & \text { G. S. Mailen } \\ 25-29 . & \text { J. W. Miche1 }\end{aligned}$
30-44. S. L. Milora
45. R. W. Murphy
46. H. Postma
47. R. C. Robertson
48. M. W. Rosenthal
49. M. R. Sheldon
50. I. Spiewak
51. D. G. Thomas
52. D. B. Trauger
53. G. D. Whitman
54. W. J. Wilcox
55-58. Central Research Library
59. Document Reference Section
60-61. Laboratory Records
62. Laboratory Records - RC
63. ORNL Patent Office

\section{EXTERNAL DISTRIBUTION}

64. Director, Division of Geothermal Energy, ERDA, Washington, DC 20545

65. Assistant Director for Resource Utilization, Division of Geothermal Energy, ERDA, Washington, DC 20545

66. Assistant Director for Advanced Research \& Technology, Division of Geotherma1 Energy, ERDA, Washington, DC 20545

67. Chief, Utilization Technology Branch, Division of Geothermal Energy, ERDA, Washington, DC 20545

68. Program Manager, Utilization Technology Branch, Division of Geothermal Energy, ERDA, Washington, DC 20545

69. R. L. Aamondt, Los Alamos Scientific Laboratory, P.0. Box 1663, Los Alamos, NM 87544

70. A. L. Austin, L-505, Geothermal Program, Lawrence Livermore Laboratory, University of California, P.0. Box 808, Livermore CA 94550

71. Glen E. Brandvold, Sandia Laboratories, P.O. Box 5800, Albuquerque, NM 87115

72. R. B. Coryel1, Geothermal Program, National Science Foundation, Washington, DC 20550

73. Michael Green, Energy and Environment Division, Lawrence Berkeley Laboratory, University of California, CA 94720

74. T. C. Hinrichs, Magma Power Company, 5143 Sunset Blvd., Los Angeles, CA 92112

75. Ivonne J. Ingvarsson, Aerojet Nuclear Company, 550 Second Street, Idaho Falls, ID 83401

76. Gerald Johnson, Bechtel Coproration, P.0. Box 3965, San Francisco, CA 94119

77. G. A. Kolstad, Division of Physical Research, ERDA, Washington, DC 20545

78. J. F. Kunze, Aerojet Nuclear Company, 550 Second Street, P.0. Box 1845, Idaho Falls, ID 83401 
79. J. T. Kuwada, Rogers Engineering Company, Inc., 111 Pine Street, Sixth Floor, San Francisco, CA 94111

80. Alan D. K. Laird, Mechanical Engineering Department, University of California, Lawrence Berkeley Laboratory, Berkeley, CA 94720

81. G. W. Leonard, Code 4511, Naval Weapons Center, China Lake, CA 93555

82. A. Lundberg, L-505, University of California, Lawrence Livermore Laboratory, P.0. Box 808, Livermore, CA 94550

83. Professor J. J. Martin, Chemical Engineering Department, University of Michigan, Ann Arbor, MI 48104

84. Kenneth Mirk, Lawrence Berkeley Laboratory, University of California, Berkeley, CA 94720

85. Kevin P. Murphy, Specialty Chemicals Division, Allied Chemical Corporation, P.O. Box 1069, Buffalo, NY 14240

86. James Nugent, Geothermal Energy, San Diego Gas \& Electric Company, P.U. Box 1831, San Diego, CA 92112

87. William Ogle, 3801 West 44th Avenue, Anchorage, AK 99503

88. J. J. Perona, Chemical \& Metallurgical. Engineering Department, University of Tennessee, Knoxville, TN 37916

89. D. R. Pitts, 876 Forest Drive, Route 12, Cookeville, TN 38501

90. Morton C. Smith, Los Alamos Scientific Laboratory, P.0. Box 1663, Los Alamos, NM 87544

91. Kenneth E. Starling, College of Engineering, School of Chemical Engineering \& Materials Science, University of Oklahoma, Norman, OK 73069

92. J. W. Tester, Los Alamos Scientific Laboratory, P.0. Box 1663, Los Alamos, NM 87544

93. Ronald Walter, Battelle - Pacific Northwest Laboratory, P.0. Box 999, Richland, WA 99352

94. Director, Research \& Technical Support Division, Oak Ridge Operations Office, P.O. Box E, Oak Ridge, TN 37830

y)-121. Technical Information Center, ERDA, P.0. Box 62, Oak Ridge, TN 37830 